\title{
Integração entre múltiplas ontologias: reúso e gerência de conflitos
}

\author{
Raphael Mendes de Oliveira Cóbe
}

\author{
TESE APRESENTADA \\ $\mathrm{AO}$ \\ Instituto De Matemática e Estatística \\ $\mathrm{DA}$ \\ Universidade De SÃo Paulo \\ PARA \\ OBTENÇÃO DO TÍTULO \\ DE \\ Doutor EM CIÊNCIAS
}

Programa: Pós-Graduação em Ciência da Computação

Orientador: Prof. Dra. Renata Wassermann

Durante o desenvolvimento deste trabalho o autor recebeu auxílio financeiro da FAPESP processo 2008/10498-8

São Paulo, dezembro de 2014 


\section{Integração entre múltiplas ontologias: reúso e gerência de conflitos}

Esta é a versão original da dissertação/tese elaborada pelo candidato Raphael Mendes de Oliveira Cóbe, tal como submetida à Comissão Julgadora. 


\section{Resumo}

CÓBE, R. M. O. Integração entre múltiplas ontologias: reúso e gerência de conflitos. 2014. 120 f. Tese (Doutorado) - Instituto de Matemática e Estatística, Universidade de São Paulo, São Paulo, 2014.

A reutilização de conhecimento é uma tarefa chave para qualquer sistema computacional. Entretanto, o reúso indiscriminado desse conhecimento pode gerar resultados conflitantes com o objetivo de uso do conhecimento, levando sistemas a se comportarem de maneira imprevisível. Neste trabalho estudamos as consequências do reúso de conhecimento em ontologias baseadas em lógicas de descrição. Focamos principalmente nos problemas que podem ser causados pela fusão de ontologias. Investigamos e comparamos a capacidade das ferramentas de desenvolvimento de ontologias atuais de lidarem com esses problemas e como a teoria se desenvolveu para resolver os mesmos problemas. Realizamos a construção de um arcabouço lógico e de software, organizado na forma de um processo, que tem como objetivo auxiliar o projetista de ontologias a resolver conflitos advindos da fusão. O processo agrupa tarefas descritas normalmente na literatura em separado. Acreditamos que a união dessas abordagens leva a uma melhor solução de conflitos. Durante o desenvolvimento deste trabalho, concentramos nossos esforços principalmente no desenvolvimento de algoritmos para a construção de sub-ontologias maximais, onde os conflitos não ocorram, bem como a ordenação desses conjuntos segundo critérios comuns discutidos na literatura. Tais estratégias foram implementadas em software e testadas utilizando dados gerados automaticamente e dados reais.

Palavras-chave: ontologias, fusão, lógica de descrição. 


\section{Abstract}

CÓBE, R. M. O. Multiple ontology integration: reuse and conflict management. 2014. 120 f. Tese (Doutorado) - Instituto de Matemática e Estatística, Universidade de São Paulo, São Paulo, 2014.

Knowledge reuse is a key task during any system development. Nevertheless, careless knowledge reuse may generate conflicting outcomes regarding the system goal, leading such systems to unpredictable behaviour. With that in mind, during this research we studied the consequences of knowledge reuse in ontologies based on description logics. We focused mainly on conflicts arising from ontology merging. We investigated and compared the features developed for this purpose on ontology development tools and how the theory field proposed to deal with the same issues. We developed both a logical and a software framework grouped into a process that aims to help the ontology designer solve conflicts arising from ontology merging. The process groups common tasks that are normally described separately. We believe that the unification of these approaches should result in a better solution for the merging conflicts. We concentrated our efforts during this work on building algorithms for building maximal sub-ontologies where such conflicts are non-existent as well as means for ordering such sets according to a few relevance criteria commonly described at the literature. Such algorithms were implemented and tested against automatically generated and real data.

Keywords: ontologies, merging, description logics. 


\section{Sumário}

$\begin{array}{ll}\text { Lista de Figuras } & \text { ix }\end{array}$

$\begin{array}{ll}\text { Lista de Tabelas } & \text { xiii }\end{array}$

1 Introdução $\quad \mathbf{1}$

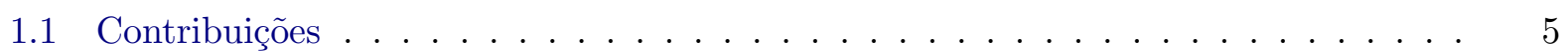

1.1 .1 Publicações . . . . . . . . . . . . . . . . . . 6

1.2 Definições . . . . . . . . . . . . . . . . . . . . . . . 6

1.2 .1 Notação . . . . . . . . . . . . . . . . . . . . . 6

1.2 .2 Inconsistências Versus Incoerências ～. . . . . . . . . . . . . . . . . 7

1.2 .3 Fusão Versus Revisão e Atualização ～. . . . . . . . . . . . . . . . . . 9

1.2.4 Mapeamento Versus Fusão Versus Casamento . . . . . . . . . . . . . . . . . 9

1.3 Organização deste Documento . . . . . . . . . . . . . . . . . . . . 10

2 Revisão de Crenças $\quad 11$

2.1 Mudanças em Bases de Crenças . . . . . . . . . . . . . . . . . . . . . . . 12

2.1 .1 Contração . . . . . . . . . . . . . . . . . . . . 13

2.2 Construção de Conjuntos Kernel . . . . . . . . . . . . . . . . . . . . . . 18

2.2.1 Algoritmos para Cálculo de Um Único Elemento do Kernel . . . . . . . . . 18

2.2 .2 Otimizações durante a Fase de Expansão . . . . . . . . . . . . . . . . . . . 19

2.2 .3 Otimizações durante a Fase de Encolhimento . . . . . . . . . . . . . . 22

2.2.4 Algoritmos para Cálculo do Conjunto Kernel Completo . . . . . . . . . . . 24

2.2.5 Otimizações ao Algoritmo de Cálculo de Árvore de Cortes Mínimos . . . . 26

2.3 Construção de Conjuntos Resíduos . . . . . . . . . . . . . . . . . . . . . . 29

2.3.1 Construção de um Único Elemento do Conjunto Resíduo . . . . . . . . . . . . 30

2.3.2 Construção do Conjunto Resíduo Completo . . . . . . . . . . . . . . . . . . 32

2.4 Ligação entre a Fusão e a Revisão de Ontologias . . . . . . . . . . . . . . . . . . . 32

3 Processo para Resolução de Conflitos em Ontologias Pós-Fusão 33

3.1 Resolução de Conflitos . . . . . . . . . . . . . . . . . . . . . . . . . . . . 34

3.2 Depuração de Granularidade Fina ～. . . . . . . . . . . . . . . . . 36

3.2 .1 Construção de Conjunto Kernel . . . . . . . . . . . . . . . . . . . . . . . . . . . . . . . . . . . . . . . .

3.2 .2 Estratificação . . . . . . . . . . . . . . . . . . . . . . . . 39

3.3 Depuração de Granularidade Grossa Completa . . . . . . . . . . . . . . . . . . . . . . . . . . . . . . . . . .

3.3.1 Construção de Conjunto Resíduo . . . . . . . . . . . . . . . . . . . . 45 
3.3.2 Estratificação . . . . . . . . . . . . . . . . . . . . . 45

3.4 Depuração de Granularidade Grossa Parcial ～. . . . . . . . . . . . . . . . . . 51

3.4.1 Construção Guiada de Um Elemento do Conjunto Resíduo . . . . . . . . . . 52

3.5 Enfraquecimento de Axiomas . . . . . . . . . . . . . . . . 54

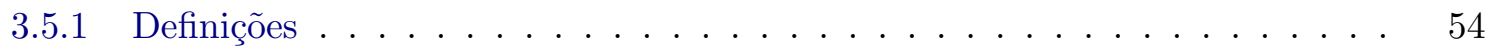

3.5.2 Axiomas de Granularidade Fina . . . . . . . . . . . . . . . . . 54

3.5.3 Calculando Justificativas de Granulação Fina . . . . . . . . . . . . . . . . 56

3.5 .4 Adição de Exceções . . . . . . . . . . . . . . . . . . . . . . . . . . 59

3.5.5 Enfraquecimento de Restrições de Cardinalidade . . . . . . . . . . . . . . . 61

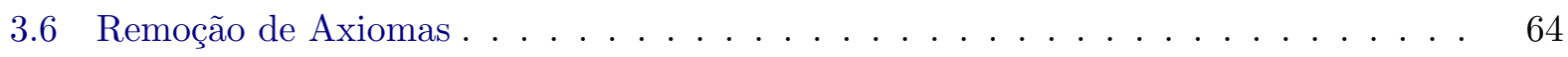

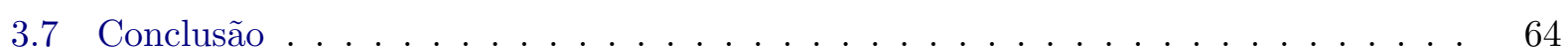

4 Detalhamento das Construções do Conjunto Resíduo $\quad 65$

4.1 Otimizações Durante a Fase de Encolhimento . . . . . . . . . . . . . . . . 65

$4.1 .1 \quad$ Janelas Deslizantes . . . . . . . . . . . . . . . . . . . . 65

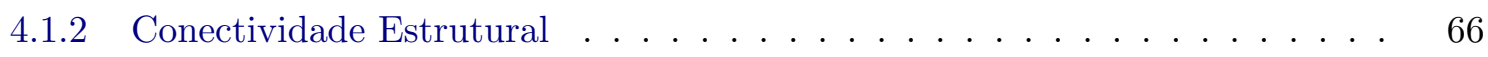

4.2 Otimizações Durante a Fase de Expansão . . . . . . . . . . . . . . . . . . . . . 67

4.2 .1 Divisão e Conquista . . . . . . . . . . . . . . . . . . . . 67 67

4.3 Arcabouço para Definição de Operadores para Cálculo de Conjuntos Resíduo e Kernel 68

4.3 .1 Construção do Conjunto Kernel e Resíduo . . . . . . . . . . . . . . . . . . . . . . . . . . . . . . . . . . . . . . .

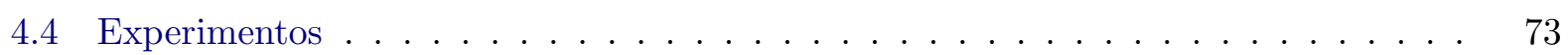

4.4 Hipóteses . . . . . . . . . . . . . . . . . . . . . 73

4.4 .2 Métricas Coletadas . . . . . . . . . . . . . . . . . . . . . 74

4.4 .3 Design do Experimento . . . . . . . . . . . . . . . . . . 74

4.4 .4 Validação dos Resultados . . . . . . . . . . . . . . . . . 75

4.4.5 Ambiente de Execução do Experimento . . . . . . . . . . . . . . . . . . . . . 75

4.4 .6 Dados Gerados . . . . . . . . . . . . . . . . . . . 76

4.4 Dados do BioPortal . . . . . . . . . . . . . . . . . . . . . . . 91

4.5 Chamadas ao Mecanismo de Inferência . . . . . . . . . . . . . . . . . . . . . . 94

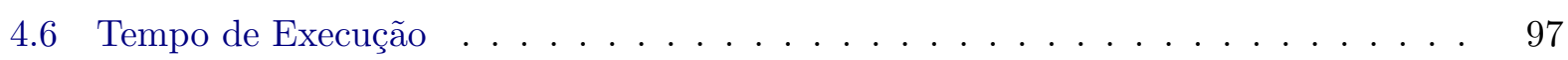

4.7 Conclusão . . . . . . . . . . . . . . . . . . . . . . . . . 100

5 Considerações Finais $\quad 101$

5.1 Considerações Finais e Contribuições . . . . . . . . . . . . . . . . . . . . . . . 101

5.2 Trabalhos Futuros . . . . . . . . . . . . . . . . . . . . 103

$\begin{array}{ll}\text { A Ferramentas para Fusão de Ontologias } & 105\end{array}$

A.1 Depuração de Ontologias . . . . . . . . . . . . . . . . . . . . . . 105

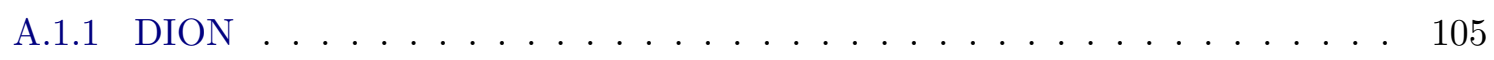

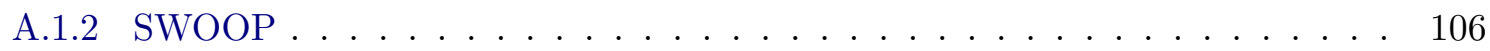

A.1.3 Neon Toolkit + RADON Plugin . . . . . . . . . . . . . . . 108

A.1.4 Protégé + OWLExplanation Plugin . . . . . . . . . . . . . . . . 110

A.2 Ferramentas para Reuso de Ontologias . . . . . . . . . . . . . . . . . . . . . . . . . . . . . . . 115

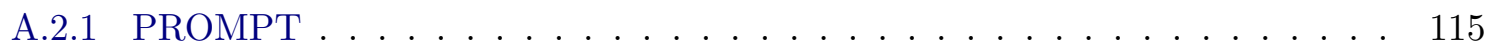


A.2.2 Protégé Merge Ontologies Refactor . . . . . . . . . . . . . . . . 120

A.2.3 Watson for Knowledge Reuse . . . . . . . . . . . . . . . . . 121

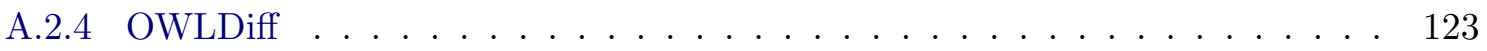

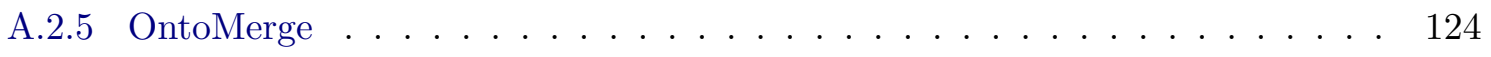

Referências Bibliográficas $\quad 125$ 


\section{Lista de Figuras}

1.1 As classes Class1 e Class2 são disjuntas . . . . . . . . . . . . . . . . . . . . 8

1.2 Ao fazermos uso de um mecanismo de inferências, descobrimos uma incoerência . . 8

1.3 Mensagem apresentada pelo editor ao se trabalhar com ontologias inconsistentes. . 8

1.4 Diferença entre casamento e fusão de ontologias - casamento de conceitos . . . . . 10

1.5 Diferença entre casamento e fusão de ontologias - fusão de conceitos . . . . . . . . 10

2.1 Árvore de cortes mínimos - Exemplo 2.6. . . . . . . . . . . . . . . . . . . . . 26

2.2 Árvore de cortes mínimos - Exemplo 2.7 . . . . . . . . . . . . . . . . . . 27

2.3 Árvore de cortes mínimos - Fechamento do nó 10 . . . . . . . . . . . . . . . 28

2.4 Árvore de cortes mínimos - Fechamento do nó 10 e reuso do nó 6 . . . . . . . . . . . 29

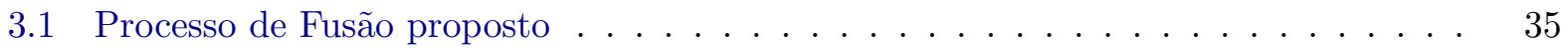

3.2 Processo de fusão proposto para o caso de depuração de granularidade fina $\quad$. . . . 37

3.3 Processo de fusão proposto para o caso de depuração de granularidade grossa com o conjunto resíduo completo . . . . . . . . . . . . . . . . . . . . 44

3.4 Processo de fusão proposto para o caso de depuração de granularidade grossa com o conjunto resíduo parcial . . . . . . . . . . . . . . . . . . 53

4.1 Arquitetura Geral do Framework . . . . . . . . . . . . . . . . . . . . . . . . . . 69

4.2 Arquitetura de Componentes para a construção de Elementos do Conjunto Kernel 70

4.3 Arquitetura de Componentes para a construção de Elementos do Conjunto Resíduo 71

4.4 Arquitetura do arcabouço com componentes para a construção de kernel e resíduo

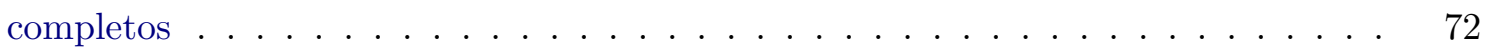

4.5 Comparativo entre algoritmos para construção do conjunto Kernel - Chamadas ao mecanismo de inferências . . . . . . . . . . . . . . . . . . 77

4.6 Comparativo entre algoritmos para construção do conjunto Kernel - tempo de execução 78

4.7 Comparativo entre algoritmos para construção do conjunto Kernel - uso de memória 78

4.8 Comparativo entre algoritmos para construção do conjunto Resíduo - Chamadas ao mecanismo de inferências . . . . . . . . . . . . . . . . . . 80

4.9 Comparativo entre algoritmos para construção do conjunto Resíduo - tempo de execução . . . . . . . . . . . . . . . . . . . . . . . . 81

4.10 Comparativo entre algoritmos para construção do conjunto Resíduo - uso de memória 81

4.11 Comparativo entre algoritmos para construção dos conjuntos Kernel e Resíduo Chamadas ao mecanismo de inferências 
4.12 Comparativo entre algoritmos para construção dos conjuntos Kernel e Resíduo Tempo de execução . . . . . . . . . . . . . . . . . . . . . . . .

4.13 Comparativo entre algoritmos para construção dos conjuntos Kernel e Resíduo - Uso

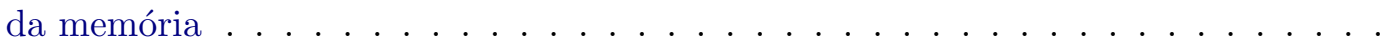

4.14 Comparativo entre algoritmos para construção do conjunto Kernel - Chamadas ao mecanismo de inferências . . . . . . . . . . . . . . 85

4.15 Comparativo entre algoritmos para construção do conjunto Kernel - tempo de execução 86

4.16 Comparativo entre algoritmos para construção do conjunto Kernel - uso de memória 86

4.17 Comparativo entre algoritmos para construção do conjunto Resíduo - Chamadas ao mecanismo de inferências . . . . . . . . . . . . . . .

4.18 Comparativo entre algoritmos para construção do conjunto Resíduo - tempo de

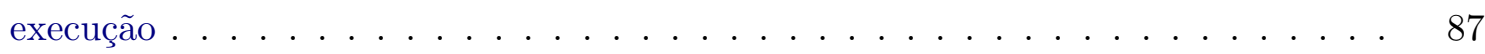

4.19 Comparativo entre algoritmos para construção do conjunto Resíduo - uso de memória 88

4.20 Comparativo entre algoritmos para construção dos conjuntos Kernel e Resíduo Chamadas ao mecanismo de inferências _ . . . . . . . . . . . . . . . . 89

4.21 Comparativo entre algoritmos para construção dos conjuntos Kernel e Resíduo Tempo de execução . . . . . . . . . . . . . . . . . . . . .

4.22 Comparativo entre algoritmos para construção dos conjuntos Kernel e Resíduo - Uso da memória . . . . . . . . . . . . . . . . . . . . . . . 90

A.1 Interface da ferramenta SWOOP para cálculo do conjunto Kernel . . . . . . . . . . 106

A.2 Interface para construção de reparos para incoerências . . . . . . . . . . . . . . 107

A.3 Interface para cálculo de justificativas para inferências . . . . . . . . . . . . . 108

A.4 Interface do Plugin RaDON do Neon Toolkit . . . . . . . . . . . . . . . . 109

A.5 Cálculo de Justificativas no RaDON . . . . . . . . . . . . . . . . . . . . . . . 109

A.6 Cálculo da nota do Reparo de uma incoerência . . . . . . . . . . . . . . . 110

A.7 Interface da ferramenta OWLExplanation - Justificativa para Incoerência . . . . 111

A.8 Interface da ferramenta OWLExplanation - Justificativa para Inconsistência . . . . 111

A.9 OWLExplanation - Cálculo de Justificativas . . . . . . . . . . . . . . . . . . . . 112

A.10 OWL-explanation - Cálculo de Justificativas Lacônicas . . . . . . . . . . . . . . 113

A.11 Fusão no PROMPT - entradas ～. . . . . . . . . . . . . . . . . . 117

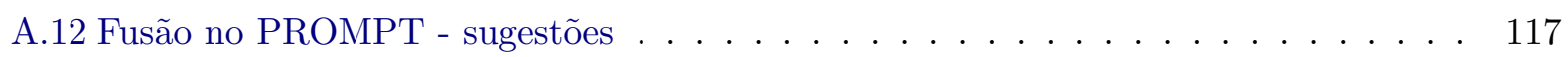

A.13 Fusão no PROMPT - ontologia pós fusão ～. . . . . . . . . . . . . . . . . 117

A.14 Fusão no PROMPT - cadastramento de operação de fusão de classes . . . . . . . . 117

A.15 Conflito de nome . . . . . . . . . . . . . . . . . . . . 118

A.16 Conflito de Referências Pendentes . . . . . . . . . . . . . . . . . . 118

A.17 Redundância na hierarquia de classes - exemplo . . . . . . . . . . . . . . . . . 119

A.18 Redundância na hierarquia de classes - sugestão do PROMPT . . . . . . . . . . . 119

A.19 Valores de propriedades violando restrições de valores de propriedades - cenário . . 119

A.20 Valores de propriedades violando restrições de valores de propriedades - cópias em separado de conceitos que ameaçam a consistência . . . . . . . . . . . . . . 120

A.21 Seleção de Ontologias para realizar Fusão . . . . . . . . . . . . . . . . . . . 120

A.22 Erro ao tentar realizar Fusão de ontologias com mesmo IRI . . . . . . . . . . . . . 121

A.23 Sugestão de inclusão de relação feita pela Watson . . . . . . . . . . . . . . . . . . 122 
A.24 Ontologia desenvolvida pelo usuário . . . . . . . . . . . . . . . . . 122

A.25 Sugestão de inclusão de axioma à Ontologia desenvolvida pelo usuário . . . . . . . . 123

A.26 Ontologias a passarem pela fusão utilizando OWLDiff . . . . . . . . . . . . . . . 123

A.27 Inconsistência gerada pela operação de fusão realizada pelo OWLDiff . . . . . . . . 123 


\section{Lista de Tabelas}

4.1 Ontologias Utilizadas no Experimento . . . . . . . . . . . . . . . . . . . . 92

4.2 Nomes dos operadores . . . . . . . . . . . . . . . . . . . . . 94

4.3 Resumo do Experimento - Chamadas ao Mecanismo de Inferência . . . . . . . . . . 95

4.4 Resumo do Experimento - Tempo de Execução . . . . . . . . . . . . . . . . . . . 98 


\section{Capítulo 1}

\section{Introdução}

A tarefa de construir uma ontologia ainda é algo custoso e em boa parte dos casos necessita de especialistas no domínio em questão. Os programas editores ainda estão em fase de amadurecimento. Tendo isso em mente, um dos principais objetivos desse trabalho é estudar formas de reutilizar partes de conhecimento prévio, facilitando a tarefa de construção de ontologias de domínio. Focamos, em especial na fusão de ontologias, mais especificamente na fusão segura. Nesse contexto, a fusão segura se dá quando duas ontologias sofrem fusão e essa operação não implica na ocorrência de inferências indesejadas - inferências não previstas pelo projetista e que não condizem com a realidade do domínio modelado.

A construção de um arcabouço para a depuração de ontologias pós-fusão é dificultada pelo baixo nível de integração entre trabalhos que lidam com a fusão de ontologias do ponto de vista teórico e do prático. Por um lado, muito do que é descrito na literatura não se encontra implementado ou integrado a ferramentas de desenvolvimento de ontologias. Por outro, as ferramentas que estão disponíveis não abordam os possíveis problemas lógicos que podem ocorrer após a fusão de ontologias.

Nesse contexto, vale a pena diferenciar os conceitos de fusão, atualização e revisão de conhecimento. Se considerarmos uma ontologia como uma base de conhecimento, onde vários pedaços de conhecimento se encontram definidos (axiomas), durante a atividade de fusão, os pedaços de conhecimento contidos em todas as bases são considerados igualmente importantes ou confiáveis. Durante as atividades de atualização e revisão, o novo conhecimento a ser incluído deve ser mais importante que o conhecimento prévio [Rev97].

A preocupação com a fusão de conhecimento do ponto de vista teórico não é nova e muitos autores se propuseram a resolver o problema em outras lógicas, como por exemplo a lógica proposicional. Para lidar com o problema de informações conflitantes nessa lógica, muitos autores [KPP98, Rev93] utilizam a abordagem de selecionar modelos que estão minimamente distantes dos modelos de cada uma das bases proposicionais. Isto pode ser feito minimizando o nível de insatisfação global, o que é conhecido como majoração ou minimizando o nível de insatisfação de cada base, o que é conhecido como arbitragem. O primeiro dos métodos garante que se a maioria das bases concordam em um determinado modelo, esse modelo vai estar entre os modelos da base pós-fusão, ao passo que o segundo método garante que a média é utilizada, satisfazendo ao máximo cada uma das bases [KPP99, Kon00].

No contexto da lógica de descrição, os estudos que abordam o problema da resolução de inconsistências podem ser classificados em dois grandes grupos $\left[\mathrm{HvHH}^{+} 05\right]$ : estudos que abordam o 
problema de um ponto de vista semântico, onde ontologias são vistas como um conjunto de modelos que são objetos semânticos e que são representados por um conjunto finito de axiomas; e estudos que abordam o problema de um ponto de vista puramente sintático, onde ontologias são vistas como conjuntos de axiomas, que são objetos estritamente sintáticos.

Do ponto de vista sintático, os autores buscam formas de construir conjuntos de axiomas que são livres de inconsistências e a obtenção desses conjuntos se dá através de operações sintáticas sobre as ontologias que estão sofrendo fusão, por exemplo removendo e adicionando axiomas. Esse tipo de estratégia foi empregada em [MLB05, RRW14] e em [HvHH+ 05, Kal06, SHCvH07]. No primeiro grupo, os autores constroem conjuntos maximalmente consistentes, chamados conjuntos resíduo ou remainder, removendo os axiomas geradores de conflitos de forma a manter o maior número de axiomas possível. No segundo grupo, os autores seguem o caminho oposto, construindo conjunto minimalmente conflitantes, chamados conjuntos minimais de conflitos ou kernel [Rei87, Was99].

A construção do conjunto kernel é um tópico bem estudado e algumas otimizações foram propostas para a sua geração, com o objetivo de minimizar o número de chamadas ao mecanismo de inferência e a redução do tempo necessário, quer seja para a construção de um único elemento como a construção do conjunto inteiro, como pode ser visto em [Kal06, SQJH08, KPHS07, JQH09, Hor11].

O mesmo estudo não é visto na área da construção do conjunto resíduo, sendo [RRW14] uma das poucas referências a construção direta do conjunto resíduo, que normalmente é obtido a partir do algoritmo de Reiter [Rei87], durante a construção do conjunto kernel completo. Nesse trabalho propomos um conjunto de heurísticas, inspiradas nas otimizações para a construção do conjunto kernel. Realizamos também experimentos para comprovar que essas heurísticas são eficazes para a construção de elementos do conjunto resíduo.

Do ponto de vista semântico, muitas pesquisas foram inspiradas pelos trabalhos conduzidos na área de resolução de conflitos baseada em modelos para lógica proposicional como [Kon00, Rev93, Dal88], onde os autores muitas vezes buscam formas de calcular modelos minimamente distantes dos modelos individuais de cada base de conhecimento que está sofrendo fusão. A ideia é que o conjunto de axiomas correspondente ao modelo mais próximo aos modelos individuais seja uma solução para a fusão. Essa tarefa é dificultada quando lidamos com lógicas de primeira ordem, uma vez que axiomas simples podem possuir infinitos modelos. Assim, diversos autores como Gorogianis e Hunter [GH08], Qi et al. [QLB06], Horridge [Hor11] e Kalyanpur [Kal06] propuseram estratégias baseadas em modificações de axiomas presentes em bases que estão sofrendo fusão para lidar com problemas de modelagem surgidos após a fusão de ontologias.

Gorogianis e Hunter, em [GH08], propõem uma estratégia de modificação iterativa através da substituição de quantificadores universais por existenciais. Eles aplicam essa ideia a lógica de predicados, e.g. a fórmula $\forall x . p(x) \rightarrow z(x)$ seria transformada em $\exists x . p(x) \rightarrow z(x)$. A essa estratégia os autores dão o nome de operadores de dilatação. Definindo várias estratégias de dilatação, poderíamos realizar um paralelo à lógica proposicional, considerando a distância entre os possíveis reparos para uma inferência indesejada em termos do número de dilatações necessárias para invalidá-la. Ou seja, com essa abordagem os autores puderam utilizar toda a infra-estrutura estabelecida para resolução de conflitos baseada em modelos de lógicas proposicionais, definindo ordenações sobre a quantidade de transformações necessárias para se obter a consistência, selecionando assim a alternativa com mudança mínima. O quesito de mudança mínima nesse caso é discutível. Na lógica proposicional, a mudança mínima é vista como variação no modelo que satisfaz o conjunto de fórmulas. No caso 
da dilatação proposta, não podemos garantir que o modelo foi mudado minimamente.

Em Qi et al. [QLB06], os autores propõem uma abordagem baseada em exceções para a resolução de conflitos em asserções e para a definição dessas exceções eles fazem uso de uma construção presente em algumas lógicas DL chamada de nominais, através da qual é possível fazer referência a um conjunto de indivíduos e tratá-los como um conceito, e.g., a fórmula $C \sqsubseteq D$ poderia ser enfraquecida em $\left(C \sqcap\left\{\neg a_{1}\right\} \sqcap\left\{\neg a_{2}\right\} \sqcap \ldots \sqcap\left\{\neg a_{n}\right\}\right) \sqsubseteq D$, onde quanto menor o número de indivíduos adicionados a fórmula enfraquecida, menor a modificação. Dessa forma, os autores também conseguiram manter a ideia de minimização de mudanças. Novamente são realizadas mudanças sintáticas na base, esperando que os novos axiomas reflitam mudanças mínimas no modelo para a ontologia. No caso de Qi et al., os autores propõem uma forma de enfraquecimento de axiomas, onde um axioma $\alpha_{1}$ é menos fraco que outro axioma $\alpha_{2}$ se e somente $\alpha_{1} \models \alpha_{2}$ e $\alpha_{2} \not \models \alpha_{1}$. Essa forma de modificação evita o enfraquecimento de um conjunto de axiomas, via remoção de axiomas, enfraquecendo axiomas em si.

Nos trabalhos de Kalyanpur [Kal06] e Horridge [Hor11] os autores propõem as funções split e $\delta$ para a modificação sintática de axiomas, dividindo-os em partes mais enfraquecidas. Ambas funções constroem extensões conservativas do conjunto sendo enfraquecido, i.e., sendo um modelo $\mathcal{M}$ para uma ontologia $\mathcal{O}, \mathcal{O}^{\prime}$ é uma extensão conservatória de $\mathcal{O}$ se todo modelo que satisfaz $\mathcal{O}^{\prime}$ também satisfaz $\mathcal{O}$ e todo modelo de $\mathcal{O}$ pode ser estendido para um modelo de $\mathcal{O}^{\prime}$, escolhendo a interpretação apropriada para os novos conceitos introduzidos em $\mathcal{O}^{\prime}$. Essa técnica é utilizada para a depuração de ontologias, quando se constroem Justificativas para uma dada inferência, i.e., um conjunto minimal (com relação a inclusão de conjuntos) de axiomas, que quando se encontram juntos, a inferência é válida.

À junção das funções que constroem extensões conservativas de ontologias para obtenção de justificativas é dado o nome de Justificativa de Granularidade Fina. Ao construirmos as justificativas a partir dessas extensões conservativas, evitamos os chamados problemas de mascaramento. Nesse fenômeno, justificativas alternativas ou inferências são mascaradas por outras justificativas. Uma das formas do fenômeno pode ser visto no Exemplo 1.1.

Exemplo 1.1 Suponha que tenhamos a ontologia:

$$
\begin{aligned}
\mathcal{O}=\{A & \sqsubseteq B \sqcap \neg C, \\
A & \sqsubseteq \neg B \sqcap C\} \models A \sqsubseteq \perp
\end{aligned}
$$

A partir dessa ontologia temos um único conjunto minimalmente conflitante (incoerente):

$$
\mathcal{K}=\{A \sqsubseteq B \sqcap \neg C, A \sqsubseteq \neg B \sqcap C\}
$$

Entretanto, intuitivamente vemos duas formas de justificar a incoerência: $\mathcal{J}_{1}=\{A \sqsubseteq B \sqcap \neg B\}$ e $\mathcal{J}_{2}=\{A \sqsubseteq C \sqcap \neg C\}$. A intuição de que existem duas formas de provar que $A \sqsubseteq \perp$ só é capturada quando os axiomas na ontologia são enfraquecidos, obtendo: 


$$
\begin{aligned}
\mathcal{O}^{\prime}=\{A & \sqsubseteq B, \\
A & \sqsubseteq \neg B, \\
A & \sqsubseteq C, \\
A & \sqsubseteq \neg C\}
\end{aligned}
$$

Nesse exemplo, a base enfraquecida é logicamente equivalente a base original. A forma de mascaramento mostrada no Exemplo 1.1 é classificada por Horridge como sendo mascaramento cruzado, no qual, a junção de partes de axiomas unidas criam novas justificativas.

Assim como a adição de exceções [QLB06], a aplicação de técnicas de enfraquecimento de axiomas tem como objetivo realizar modificações menores ao conjunto de modelos para a ontologia sendo enfraquecida, do que a remoção completa de axiomas.

Novamente, essas técnicas são propostas e aplicadas a depuração e reparo centrados na construção do conjunto kernel. Nesse trabalho, propomos a aplicação dessas técnicas para depuração e reparo baseado em conjuntos resíduos.

Uma característica bastante importante que afeta tanto os trabalhos que propõem estratégias de resolução de conflitos sintáticas ou semânticas para ontologias é assumir que as ontologias que estão sofrendo fusão podem ser ordenadas de acordo com a preferência ou confiabilidade dos axiomas nelas contidos. O processo de ordenação dos axiomas dentro de ontologias é chamado de estratificação. Esse processo cria subconjuntos na ontologia chamados de estratos - strata, onde dentro de cada estrato os axiomas possuem o mesmo nível de confiança.

O processo de estratificação é tipicamente realizado utilizando uma estratégia manual, onde o projetista da ontologia define quais axiomas são mais relevantes para o domínio sendo descrito. Entretanto, essa tarefa pode ser bastante custosa e de difícil execução em casos que as bases que estão sofrendo fusão são de tamanhos consideráveis. Para lidar com conjuntos grandes, autores como Qi et al., em [QP07], Haase et al., em [HvHH+05] e Kalyanpur, em [Kal06] propuseram formas automáticas de estratificar ontologias.

Qi et al., em [QP07] propôs ordenar axiomas de acordo com a especificidade de um axioma, onde um axioma $C_{1} \sqsubseteq D_{1}$ é mais específico que um outro $C_{2} \sqsubseteq D_{2}$, se e somente se $C_{1} \sqsubseteq C_{2}$ e $C_{2} \nsubseteq C_{1}$. Com essa forma de estratificação, o autor dá maior prioridade a axiomas que descrevem conceitos mais específicos que outros.

De forma similar, Hasse et al. $\left[\mathrm{HvHH}^{+} 05\right]$ propuseram o agrupamento de axiomas em estratos de acordo com a proximidade (sintática) desses axiomas a um conceito que seja inconsistente. Eles fazem uso de uma ideia de conectividade sintática entre conceitos que informa quão conectado a um conceito inconsistente um determinado conceito se encontra. Dessa forma, o autor classifica conceitos como menos relevantes se eles estiverem mais próximos (sintaticamente) de um axioma envolvido em um conflito.

Kalyanpur, em [Kal06], propôs algumas formas de estratificação para uma ontologia, centradas na ideia de um conjunto minimalmente inconsistente ou incoerente de uma ontologia. O trabalho em questão é focado na depuração de uma ontologia inconsistente/incoerente, onde deseja-se resolver esse conflito lógico, removendo o mínimo necessário. A escolha pelo que remover é o que motiva o autor a definir as estratégias baseadas em: a) frequência: nesse caso a informação a ser removida 
é a que se encontra no maior número de conflitos; $b$ ) relevância sintática: o axioma removido é aquele menos utilizado (sintaticamente) na ontologia; $c$ ) relevância semântica: o axioma removido é aquele que causa o menor número de perdas de inferências na ontologia.

Essas técnicas de estratificação como propostas são aplicadas na depuração de ontologias baseada em conjuntos kernel. Acreditamos que essas técnicas são também úteis na depuração e reparo centrados em conjunto resíduo. Para aplicar tais técnicas realizamos a tradução das mesmas para o contexto do conjunto resíduo, tanto durante a depuração e reparo baseada no conjunto resíduo completo como para o parcial. No caso do conjunto resíduo completo, a estratificação é realizada após a construção do conjunto inteiro. Já no caso do conjunto resíduo parcial, a construção dos elementos é guiada pela estratégia de estratificação.

Do ponto de vista prático, os softwares desenvolvidos com o objetivo de realizar a fusão de bases de conhecimento não apresentam muito suporte a depuração de inferências indesejadas que podem ser introduzidas após o processo de fusão. Portanto, dividimos nossa análise de ferramentas em ferramentas que promovem o reúso de ontologias e ferramentas utilizadas para depuração e reparo de ontologias.

Do ponto de vista de depuração, analisamos a ferramenta $\operatorname{RaDON}^{1}$, que realiza a depuração de ontologias inconsistentes/incoerentes e sugere reparos para as mesmas; $\mathrm{DION}^{2}$, ferramenta que calcula conjuntos minimalmente inconsistentes/incoerentes e OWLExplanation Plugin, ferramenta que calcula justificativas para qualquer inferência válida na ontologia, inclusive inconsistências e incoerências.

Para ferramentas de reúso de ontologias, analisamos os softwares Watson For Knowledge Reuse ${ }^{3}$, ferramenta que consulta uma base online de ontologias e busca conceitos similares ao que está sendo descrito na ferramenta, sugerindo a inclusão de novos conceitos relacionados ao que se está sendo definido; OWLDiff ${ }^{4}$, ferramenta utilizada para mostrar a diferença estrutural entre duas ontologias, possibilitando mesclar essas ontologias em uma nova; e $\mathrm{PROMPT}^{5}$, a referência mais clássica para a fusão de ontologias. A ferramenta se propõe a lidar com alguns problemas que podem ser causados após a fusão. Infelizmente nenhum desses problemas possui uma natureza lógica. Por isso essa ferramenta está classificada como somente promovendo o reúso.

\subsection{Contribuições}

A maior contribuição desse trabalho é a definição de um processo para a depuração e reparo de ontologias pós fusão, possibilitando a reutilização segura de ontologias. Nesse processo agregamos as principais técnicas descritas na literatura para a depuração, enfraquecimento e estratificação. Dividimos a tarefa de depuração e reparo em duas formas principais: a depuração de granularidade fina, feita via conjunto kernel e a depuração de granularidade grossa, feita via elementos do conjunto resíduo, que pode ser completo - quando calculamos o conjunto inteiro -, ou parcial - com um único elemento calculado.

A depuração de granularidade fina é importante quando o projetista deseja obter todas as

\footnotetext{
${ }^{1}$ http://neon-toolkit.org/wiki/RaDON

${ }^{2}$ http://wasp.cs.vu.nl/sekt/dion

${ }^{3}$ http://neon-toolkit.org/wiki/1.x/Watson_for_Knowledge_Reuse

${ }^{4}$ http://krizik.felk.cvut.cz/km/owldiff/

${ }^{5}$ http://protege.stanford.edu/plugins/prompt/prompt.html
} 
causas possíveis para uma inferência indesejada. Em contrapartida, na depuração de granularidade grossa, o projetista deseja construir uma ontologia onde a inferência indesejada não seja válida. Essa construção pode ser guiada, onde a preferência de axiomas é levada em conta ou uma construção completa, onde todas as alternativas são mostradas.

Para o processo proposto, definimos novas formas (otimizadas) de construir elementos do conjunto resíduo, uma vez que o que está descrito na literatura [RRW14] precisava necessariamente realizar consultas ao mecanismo de inferência para todos os elementos na ontologia. Realizamos experimentos utilizando essas novas construções utilizando dados reais e gerados automaticamente, onde constatamos que as otimizações propostas foram eficazes.

No processo também propusemos estratégias para estratificação do conjunto resíduo para a escolha do melhor reparo para uma inferência indesejada. No caso da construção guiada do conjunto resíduo, adaptamos essas estratégias para que a construção da melhor resposta seja feita sem que o conjunto resíduo inteiro seja gerado.

Por fim, propusemos uma nova forma de enfraquecimento de ontologias, onde o conflito está centrado em axiomas do tipo $C \sqsubseteq \leq n . P$. Caracterizamos os casos nos quais esse conflito pode ocorrer e como a solução é construída.

\subsubsection{Publicações}

Em [CW09], apresentamos as primeiras ideias relacionadas a fusão de ontologias, mostramos como aplicar esses conceitos no domínio de sistemas de buscas baseados em ontologias. Em [CWK11], apresentamos a nossa primeira solução de software para a construção de reparos para ontologias. Essa ferramenta era baseada no algoritmo CMA-DL, proposto por Meyer et al. [MLB05]. Em [CW12], apresentamos as ideias de como utilizar a revisão kernel para realizar a fusão de ontologias. Nessa publicação mostramos a primeira versão do nosso processo. Em [CRW13] mostramos modificações realizadas ao Framework B-Contractor [LRW12], para incluir funcionalidade de revisão de ontologias.

\subsection{Definições}

Durante o levantamento bibliográfico realizado, notou-se que é importante definir alguns conceitos, já que os mesmos são muitas vezes confundidos na literatura. Essa seção visa definir de forma um pouco mais direta cada um desses conceitos para que a leitura desse texto se dê de forma menos ambígua.

\subsubsection{Notação}

Nesse trabalho lidamos com lógicas de descrição $\left[\mathrm{BCM}^{+} 03\right]$. Essa seção tem como objetivo estabelecer a notação a ser utilizada por todo o resto do documento.

Utilizamos um padrão para notação inspirado em [QP07] onde conceitos são escritos utilizando letras maiúsculas $(A, B, C, D, \ldots)$ e suas versões subscritas. Representaremos relações entre conceitos também utilizando letras maiúsculas, principalmente as letras $P$ e $R$.

Consideramos por exemplo a linguagem $\mathcal{A L C N}$. Sendo $C$ e $D$ conceitos e $R$ uma relação, as seguintes construções também são conceitos: $\neg C, C \sqcap D, C \sqcup D, \forall R . C, \exists R . C, \leq n R$ e $\geq n R$. 
Uma interpretação $\mathcal{I}=\left(\Delta^{\mathcal{I}},{ }^{\mathcal{I}}\right)$ é composta de um conjunto chamado domínio da interpretação: $\Delta^{\mathcal{I}}$ e uma função ${ }^{\mathcal{I}}$ que mapeia cada conceito $C$ para um subconjunto $C^{\mathcal{I}}$ de $\Delta^{\mathcal{I}}$, cada papel $\mathrm{R}$ em um subconjunto $R^{\mathcal{I}}$ de $\Delta^{\mathcal{I}} \times \Delta^{\mathcal{I}}$ e cada indivíduo a em um elemento $a^{\mathcal{I}} \in \Delta^{\mathcal{I}}$.

Novamente utilizando o fragmento da $\mathrm{LD} \mathcal{A L C \mathcal { N }}$, para todo conceito $C, D$ e papel $R$, as seguintes propriedades são satisfeitas $\left[\mathrm{BCM}^{+} 03\right]$ :

1. $(\neg C)^{\mathcal{I}}=\Delta^{\mathcal{I}} \backslash C^{\mathcal{I}}$

2. $(C \sqcap D)^{\mathcal{I}}=C^{\mathcal{I}} \cap D^{\mathcal{I}}$

3. $(C \sqcup D)^{\mathcal{I}}=C^{\mathcal{I}} \cup D^{\mathcal{I}}$

4. $(\exists R . C)^{\mathcal{I}}=\left\{x \in \Delta^{\mathcal{I}} \mid \exists y\right.$ t.q. $(x, y) \in R^{\mathcal{I}}$ e $\left.y \in C^{\mathcal{I}}\right\}$

5. $(\forall R . C)^{\mathcal{I}}=\left\{x \in \Delta^{\mathcal{I}} \mid \forall y\right.$ t.q. $\left.(x, y) \in R^{\mathcal{I}} \rightarrow y \in C^{\mathcal{I}}\right\}$

6. $(\leq n R)^{\mathcal{I}}=\left\{x \in \Delta^{\mathcal{I}}||\left\{y \mid(x, y) \in R^{\mathcal{I}}\right\} \mid \leq n\right\}$

7. $(\geq n R)^{\mathcal{I}}=\left\{x \in \Delta^{\mathcal{I}}||\left\{y \mid(x, y) \in R^{\mathcal{I}}\right\} \mid \geq n\right\}$

Nesse contexto, uma base de conhecimento em lógica de descrição é composta de dois componentes, um terminológico (TBox) e um assercional (ABox), onde TBox é um conjunto finito de axiomas lógicos do tipo $C \sqsubseteq D$, onde $\mathrm{C}$ e $\mathrm{D}$ são conceitos. Uma interpretação $\mathcal{I}$ satisfaz o axioma $C \sqsubseteq D$ sse $C^{\mathcal{I}} \subseteq D^{\mathcal{I}}$.

Em uma base de conhecimento em lógica de descrição também é possível descrever informações a respeito dos indivíduos. Para representar indivíduos vamos utilizar letras minúsculas $(\mathrm{a}, \mathrm{b}, \mathrm{c}, \ldots)$ e seus subscritos. Um axioma de asserção de conceitos é escrito como $C(a)$ e um axioma de asserção de relação é escrito como $R(a, b)$. Uma interpretação $\mathcal{I}$ satisfaz os axiomas $C(a)$ e $R(a, b)$ sse $a^{\mathcal{I}} \in C^{\mathcal{I}}$ e $\left(a^{\mathcal{I}}, b^{\mathcal{I}}\right) \in R^{\mathcal{I}}$.

No contexto de $\mathrm{LD}$ vamos utilizar os termos axiomas e fórmulas de forma intercambiável e para representar axiomas utilizaremos o alfabeto grego minúsculo $(\alpha, \beta, \gamma, \epsilon, \ldots)$. Uma interpretação é chamada de modelo para um dado axioma se satisfaz esse axioma e é chamada de modelo da base de conhecimento se satisfizer todos os axiomas contidos na base. Aqui também utilizaremos a mesma notação de [QP07] e definiremos o modelo de um determinado axioma $\phi$ como sendo $\mathcal{M}(\phi)$ e de uma dada base $\mathcal{O}$ como sendo $\mathcal{M}(\mathcal{O})$.

\subsubsection{Inconsistências Versus Incoerências}

O primeiro conceito a se observar é o de inconsistências lógicas. Um conjunto de fórmulas é considerado logicamente inconsistente se não existe nenhuma interpretação que possa satisfazer tal conjunto $\left[\mathrm{HvHH}^{+} 05\right]$, i.e., $\mathcal{M}(\mathcal{O})=\emptyset$ onde $\mathcal{O}$ é uma base de conhecimento contendo fórmulas em lógica de descrição.

Incoerências ocorrem quando para todas as interpretações possíveis, um determinado conceito atômico $C$, onde $C \neq \perp$, é interpretado como vazio, i.e. seja, para qualquer função de interpretação ${ }^{\mathcal{I}}$, que satisfaz uma ontologia, $C^{\mathcal{I}}=\emptyset$.

Os autores em [QP07] apontam que inconsistências são resultantes tipicamente do conhecimento assertivo da ontologia, ou seja os axiomas do tipo ABox presentes nas mesmas. Incoerências, por outro lado são resultados de axiomas do tipo TBox em ontologias. 
O exemplo mostrado nas Figuras 1.1 e 1.2 ilustra a presença de conceitos necessariamente vazios, i.e., $C l a s s 3^{\mathcal{I}}=\emptyset$. Na Figura 1.1, devemos considerar que os conceitos Class1 e Class2 são disjuntos, i.e. Class $1 \sqsubseteq \neg$ Class 2 . Ao fazermos uso de um mecanismo de inferências (reasoner), o mesmo aponta que não pode existir um indivíduo pertencente ao conceito Class3. A resposta do mecanismo de inferências pode ser vista na Figura 1.2, apontando que o conceito Class3 é um sub-conceito da classe OWL Nothing, i.e. Class $3 \sqsubseteq \perp$.

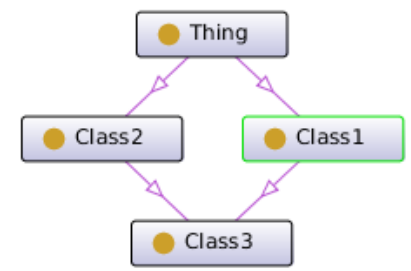

Figura 1.1: As classes Class1 e Class2 são disjuntas

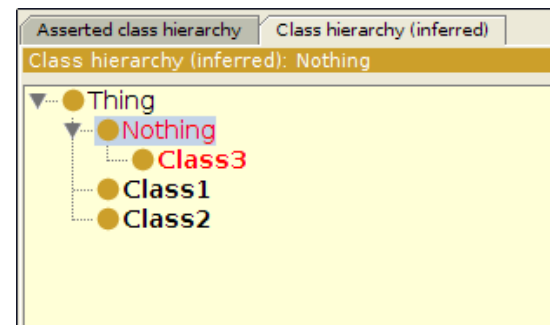

Figura 1.2: Ao fazermos uso de um mecanismo de inferências, descobrimos uma incoerência

Agora considere que a classe Class3 possui um indivíduo, i.e. Class3(Joao). Isso faz com que o reasoner não consiga achar nenhum modelo possível para essa ontologia, i.e. a função de interpretação ${ }^{\mathcal{I}}$ não consegue interpretar o conceito Joao. A Figura 1.3 mostra o resultado do reasoner ao tentar raciocinar em cima dessa ontologia inconsistente.

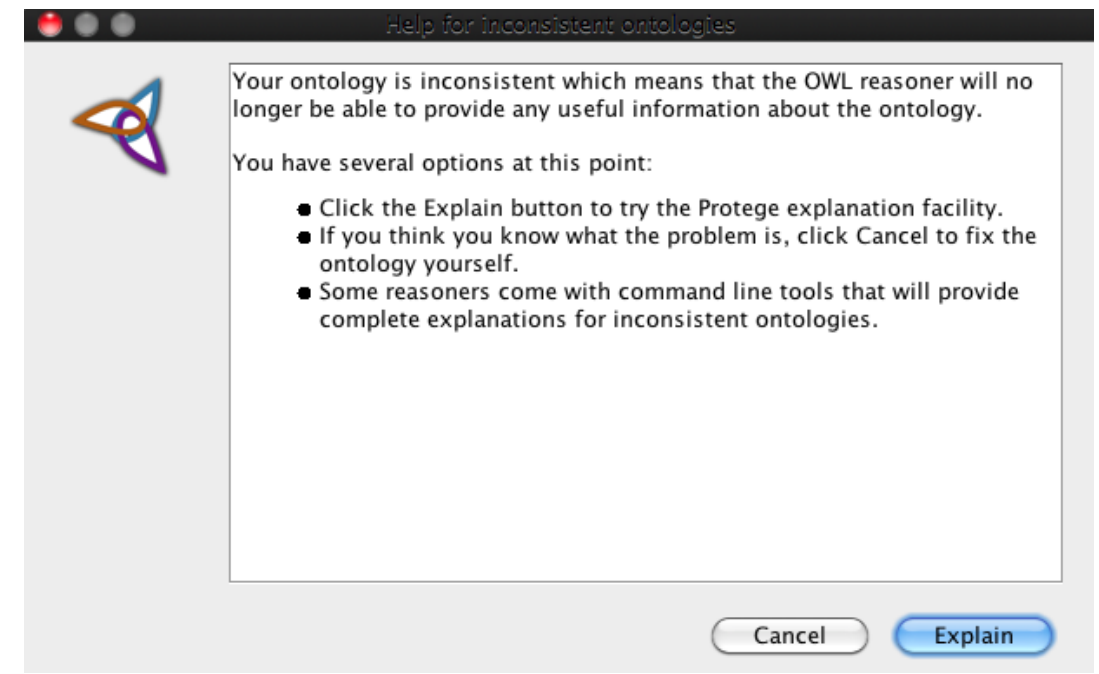

Figura 1.3: Mensagem apresentada pelo editor ao se trabalhar com ontologias inconsistentes.

Inconsistências são geradas quando indivíduos são adicionados a ontologia. Esse tipo de observação inspirou trabalhos que lidam com inconsistências através da definição de exceções, como [QP07, QLB06, MLB05]. Em [QLB06], os autores propõem uma abordagem baseada no enfra- 
quecimento gradual da operação de subordinação, explicitamente informando que determinados indivíduos não fazem parte de uma dada restrição, e.g., a classe $C_{1}$ é subordinada a classe $C_{2} \mathrm{com}$ exceção do indivíduo $a$, i.e., $\left(C_{1} \sqcap \neg\{a\}\right) \sqsubseteq C_{2}$.

\subsubsection{Fusão Versus Revisão e Atualização}

É importante ainda diferenciar alguns conceitos que muitas vezes são considerados equivalentes em trabalhos que lidam com integração e consolidação de conhecimento em ontologias. Os conceitos são: fusão (merging), atualização (update).

Nos trabalhos [Rev93, Rev97], os autores argumentam que operadores que realizam a atualização e revisão de ontologias dão prioridade a alguma parte do conhecimento, em detrimento de outra parte, ao passo que operadores que promovem a fusão não priorizam nenhum pedaço em específico. Um outro ponto interessante é notar que trabalhos que descrevem operadores de atualização e revisão geralmente tomam como entrada uma base de conhecimento e uma fórmula individual ao passo que trabalhos que propõem operadores de fusão recebem como entrada duas bases em separado. Para entender melhor essa diferenciação, vamos considerar o seguinte exemplo retirado de [MLB05]:

Exemplo 1.2 Consideremos as ontologias, $S_{1}$ composta dos seguintes axiomas: passaro(tweety), $\neg$ voa(tweety) e passaro(chirpy) e $S_{2}$ composta pelo axioma passaro $\sqsubseteq$ voa. É fácil de ver que a união dessas duas ontologias resulta em inconsistência uma vez que $S_{1}$ afirma que existe um pássaro (tweety) que não voa e $S_{2}$ afirma que todo pássaro voa.

Ao realizarmos a operação de revisão da primeira ontologia pela segunda, os axiomas da segunda teriam prioridade, ou seja, a idéia é que seus axiomas sejam mantidos no resultado da fusão. Os resultados possíveis para a revisão seriam as ontologias $S_{31}$ composta por passaro(tweety), passaro(chirpy) e passaro $\sqsubseteq$ voa; e $S_{32}$ composta por $\neg$ voa(tweety), passaro(chirpy) e passaro $\sqsubseteq$ voa.

Utilizando abordagens de fusão como a de [MLB05], o conhecimento na primeira ontologia poderia ser priorizado e para a solução da inconsistência seriam excluídos os axiomas da segunda ontologia e o resultado seria composto somente pelos axiomas presentes em $S_{1}$, ou seja: passaro(tweety), $\neg$ voa(tweety) e passaro(chirpy).

\subsubsection{Mapeamento Versus Fusão Versus Casamento}

É importante ainda diferenciar os conceitos de mapeamento (mapping), casamento (matching) e fusão (merging) de ontologias. A atividade de casamento realiza a identificação de conceitos que são, em certo nível, "equivalentes". Um exemplo de atividade de casamento é a abordagem que utiliza a análise léxica de conceitos. Um exemplo de casamento léxico pode ser visto na Figura 1.4, onde conceitos são classificados equivalentes se seus nomes assim forem. O conjunto de casamentos de conceitos é chamado de mapeamento de ontologias.

Ao considerar o processo de integração de ontologias, primeiro se faz necessário identificar a existência de conceitos que são equivalentes em certo nível ou de acordo com uma medida de confiança. Após isso feito, a fusão pode ocorrer sem que conceitos estejam repetidos. Considerando a integração como um processo, o mapeamento é a atividade que precede a fusão [FNS07]. 


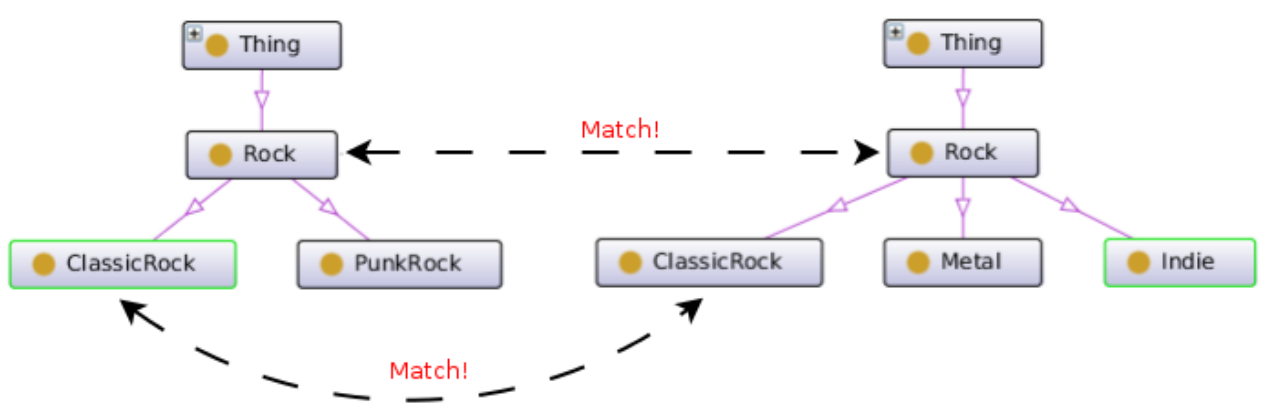

Figura 1.4: Diferença entre casamento e fusão de ontologias - casamento de conceitos

Considerando o exemplo visto na Figura 1.4, a atividade de fusão produziria a ontologia vista na Figura 1.5, onde os conceitos casados sofreram fusão e o resto da estrutura de ambas ontologias de entrada é copiado para ontologia de saída.

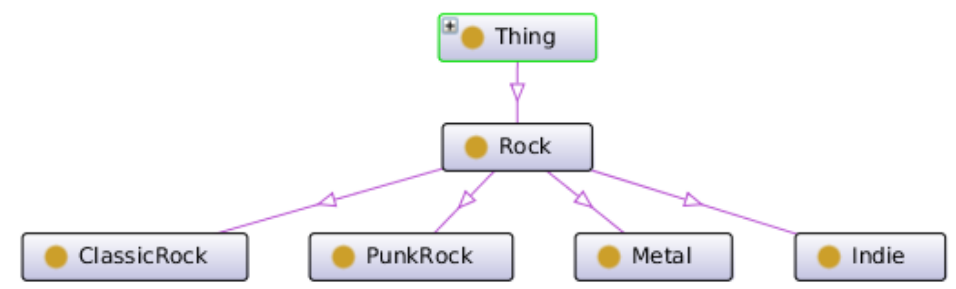

Figura 1.5: Diferença entre casamento e fusão de ontologias - fusão de conceitos

\subsection{Organização deste Documento}

Este documento está organizado da seguinte forma: No Capítulo 2 listamos alguns dos principais conceitos relacionados a área de revisão de crenças. Esses conceitos são importantes para entender as principais atividades realizadas no nosso processo proposto. Nesse capítulo detalharemos principalmente as construções de conjuntos kernel e otimizações. No Capítulo 3 introduzimos o processo que propomos para a depuração e reparo de ontologias. Explicamos cada uma das atividades e o porquê de sua adoção no processo. No Capítulo 4 mostramos as construções que propomos para a obtenção do conjunto resíduo. Mostramos também, de forma sumarizada, dados dos experimentos realizados com essas construções. Por fim, o Capítulo 5 apresenta algumas considerações sobre o trabalho desenvolvido. Nesse capítulo apresentamos também algumas ideias de trabalhos futuros. Esse texto contém também, na forma de apêndice, um estudo exploratório avaliativo de ferramentas que promovem o reuso e depuração de ontologias - Apêndice A. 


\section{Capítulo 2}

\section{Revisão de Crenças}

Esse capítulo tem como objetivo relacionar os principais conceitos utilizados na construção de nossa proposta. As principais técnicas utilizadas vêm da sub-área da Representação do Conhecimento chamada Revisão de Crenças. Em específico estamos falando da operação de Revisão de Crenças descritas utilizando lógicas de descrição.

Revisão de Crenças lida com o problema do dinamismo do conhecimento mantido por agentes, i.e., o problema de acomodar nova informação - ou crença - junto do conjunto prévio mantido pelo agente. Lida também com o problema da remoção segura de informações. Seguro do ponto de vista de garantir que uma vez que a informação seja removida, a mesma não pode ser deduzida do que restou das crenças do agente.

A Revisão de Crenças é considerada uma sub-área de um campo maior chamado Representação de Conhecimento e é baseado no paradigma AGM apresentado no artigo seminal [AGM85].

O estado epistêmico de um agente pode ser representado de diversas formas. No paradigma AGM as crenças de um agente são representadas por um conjunto de crenças, i.e., um conjunto de sentenças fechado, sob consequência lógica, ou seja, sendo $\mathcal{O}$ um conjunto de crenças e $C n$ o operador clássico de consequência lógica, então $\mathcal{O}=C n(\mathcal{O})$.

Por questões de eficiência computacional, Hansson et al. em [Han91] sugerem que sejam utilizados para a representação de crenças, bases de crenças, que são conjuntos não necessariamente fechados sob a consequência lógica. Essa simplificação torna possível lidar com conjuntos de sentenças, cujo fecho lógico implicaria em um conjunto infinito de consequências. Como consequência dessa escolha, podemos também distinguir o conhecimento explícito do conhecimento implícito, inferido a partir do conhecimento explícito.

A distinção entre essas duas formas de crenças se faz necessária para que as operações de mudanças no estado epistemológico do agente possam ocorrer de forma segura, ou seja, que a base de conhecimento não permita inferir o que não seja desejado.

Sob esse ponto de vista, a Revisão de crenças é um campo próximo a fusão de ontologias, uma vez que ambos estão preocupados com a inserção de novas informações em um conjunto pré-existente. Dessa forma, apresentaremos as principais formas de modificações no estado epistemológico de um agente, descritas na revisão de crenças. Mostraremos como a fusão de ontologias compartilha de algumas operações realizadas durante a revisão de bases de crenças. 


\subsection{Mudanças em Bases de Crenças}

Para cada fórmula $\alpha$ em uma base de conhecimento $\mathcal{O}$, podemos estabelecer que:

- $\alpha$ é aceita explicitamente se $\alpha \in \mathcal{O}$;

- $\alpha$ é aceita implicitamente se $\alpha \in C n(\mathcal{O}) \backslash \mathcal{O}$;

- $\alpha$ é rejeitada explicitamente se $\neg \alpha \in \mathcal{O}$;

- $\alpha$ é rejeitada implicitamente se $\neg \alpha \in C n(\mathcal{O}) \backslash \mathcal{O}$;

- $\alpha$ é indeterminado se $\alpha \notin C n(\mathcal{O})$ e $\alpha$ é consistente com $C n(\mathcal{O})$, i.e., $\neg \alpha \notin C n(\mathcal{O})$;

Para o resto dessa tese, consideraremos que uma fórmula $\alpha$ é aceita por uma base de crenças $\mathcal{O}$ se $\alpha$ for aceita de forma implícita ou explícita por $\mathcal{O}$. Consideraremos o mesmo princípio para o caso onde $\alpha$ não é aceita por $\mathcal{O}$.

Para a revisão de crenças, definimos 3 operações básicas para uma base de crenças, considerando uma base $\mathcal{O}$ e uma fórmula $\alpha$ : expansão $(+)$, contração (-) e revisão $\left(^{*}\right)$. A expansão é utilizada quando desejamos inserir uma nova fórmula lógica à base de crenças $(\mathcal{O}+\alpha)$. A contração garante que uma dada informação não esteja contida em uma base de crenças, nem no conhecimento implícito nem no explícito $(\mathcal{O}-\alpha)$.

A revisão no entanto é uma operação um pouco mais trabalhosa. Nela buscamos estabelecer uma forma segura de adicionar novas crenças à base de crenças do agente. A segurança da operação é vista sob o aspecto da consistência/coerência do resultado.

De acordo com Gärdenfors [Gär88], a mudança no estado epistêmico de um agente se dá a partir de um gatilho disparador. A esse gatilho é dado o nome de entrada epistêmica [Rib13].

Uma entrada epistêmica leva um agente de um estado epistêmico a outro e essas entradas podem ser:

- expansão: leva o agente do estado no qual $\alpha$ era indeterminada e agora é aceita;

- contração: leva o agente do estado onde $\alpha$ era aceita e agora é indeterminada;

- revisão: leva o agente de forma segura a aceitar $\alpha$.

O maior dos desafios para a área é definir critérios de racionalidade para operadores que causam mudanças nos estados epistêmicos dos agentes, i.e., o que devemos esperar de um agente racional ao entrar em contato com novas evidências. Por exemplo, [Har86] definiu, como critério de racionalidade, que ao realizar a entrada em um estado epistêmico, um agente deve descartar o mínimo de informação para que essa seja consistente com as crenças prévias.

Esse tipo de desafio é bem próximo do que exploramos nesse trabalho. No caso da fusão de ontologias, acreditamos que um operador também deva seguir um critério de minimalidade, de forma que podemos garantir que nenhum trecho de informação foi desnecessariamente descartado.

A operação de expansão $\mathcal{O}+\alpha$ em uma base de crenças representada por um conjunto de fórmulas é construída como $\mathcal{O}+\alpha=\mathcal{O} \cup\{\alpha\}$ [Rib13].

A operação de revisão * pode ser definida utilizando a identidade de Levi [AM82] na forma de:

$$
\mathcal{O} * \alpha=(\mathcal{O}-\neg \alpha)+\alpha
$$


Essa forma é chamada de revisão interna, onde primeiro abrimos espaço para a nova informação que será adicionada, em seguida adicionando-a. Em oposição a revisão interna, a externa é definida pelo reverso da identidade de Levi, como o que foi proposto por Hansson em [Han93]. Nessa forma de revisão primeiro adicionamos a nova informação e depois a consolidamos, realizando a contração do que for contraditório com a nova informação:

$$
\mathcal{O} * \alpha=(\mathcal{O}+\alpha-\neg \alpha)
$$

Essas operações, no paradigma AGM são regidas pelos chamados postulados de racionalidade que indicam propriedades racionais que operadores de contração, expansão e revisão devem possuir. As principais construções de operadores encontrados na literatura são equipadas com os chamados teoremas de representação. Esses teoremas mostram que uma construção caracterizada por um conjunto de postulados obedece a todos os postulados e que qualquer operador que seja caracterizado pelo mesmo conjunto de postulados, pode ser obtido a partir dessa construção [Rib13].

A seguir, vamos detalhar construções para a contração como componente básico para a definição da revisão.

\subsubsection{Contração}

Assim como no paradigma AGM, a contração em bases de crença é regida por um conjunto mínimo de postulados [Rib13]:

1. (sucesso) $\alpha \notin C n(\emptyset) \Rightarrow \alpha \notin C n(\mathcal{O}-\alpha)$

2. (inclusão) $\mathcal{O}-\alpha \subseteq \mathcal{O}$

3. (core-retainment) Se $\beta \in \mathcal{O}$ e $\beta \notin \mathcal{O}-\alpha$ então existe $\mathcal{O}^{\prime}$ t.q. $\mathcal{O}^{\prime} \subseteq \mathcal{O}$ e $\mathcal{O}^{\prime} \not \models \alpha$, porém $\mathcal{O}^{\prime} \cup\{\beta\} \vdash \alpha$

4. (relevância) Se $\beta \in \mathcal{O}$ e $\beta \notin \mathcal{O}-\alpha$ então existe $\mathcal{O}^{\prime}$ t.q. $\mathcal{O}-\alpha \subseteq \mathcal{O}^{\prime} \subseteq \mathcal{O}$ e $\mathcal{O}^{\prime} \not \neq \alpha$, porém $\mathcal{O}^{\prime} \cup\{\beta\} \vdash \alpha$

5. (uniformidade) Se é verdade que para todos os subconjuntos $\mathcal{O}^{\prime}$ de $\mathcal{O}$ vale que $\alpha \in C n\left(\mathcal{O}^{\prime}\right)$ sse $\beta \in C n\left(\mathcal{O}^{\prime}\right)$, então $\mathcal{O}-\alpha=\mathcal{O}-\beta$

O postulado do sucesso garante que, sendo contração uma operação que leva uma fórmula $\alpha$ a não ser aceita, essa operação só é bem sucedida se, após a execução do operador temos que $\alpha \notin \mathcal{O}-\alpha$, isso caso $\alpha$ não seja uma tautologia, i.e., $\alpha \notin C n(\emptyset)$.

O postulado da inclusão define que, ao executar uma contração, novas sentenças não são incluídas.

Os postulados de core-retainment e relevância são utilizados para definir critérios de minimalidade. O objetivo de ambos é garantir que operadores de contração removam um conjunto de fórmulas $\beta$, somente se essas fórmulas forem utilizadas para a inferência de $\alpha$.

Por fim, o postulado da uniformidade garante que, sendo duas sentenças equivalentes em relação a $\mathcal{O}$, i.e., são consequência dos mesmos subconjuntos $\mathcal{O}^{\prime}$; essas devem ser contraídas de um mesmo conjunto de crenças de forma equivalente.

Por termos dois critérios distintos de minimalidade, mostraremos nas próximas seções duas construções para operadores de contração, a chamada contração partial meet e a contração kernel. 


\subsubsection{Contração Kernel}

Esse tipo de construção faz uso da ideia de encontrar conjuntos minimais que implicam $\alpha$ e então remover pelo menos um elemento de cada um desses conjuntos, invalidando a inferência $\alpha$. Ao conjunto desses conjuntos minimais damos o nome de conjunto kernel de $\mathcal{O}$ por $\alpha$, utilizaremos a notação $\mathcal{O} \Perp \alpha$ para representar essa operação.

Definição 2.1 (Conjunto Kernel) [Han94]

Seja $\mathcal{O}$ uma base de crenças e $\alpha$ uma sentença. Um conjunto $\mathcal{O}^{\prime}$ é um elemento pertencente a $\mathcal{O} \Perp \alpha$ sse $\mathcal{O}^{\prime}$ é um subconjunto minimal de $\mathcal{O}$ que implica $\alpha$ :

- $\mathcal{O}^{\prime}$ é um subconjunto de $\mathcal{O}\left(\mathcal{O}^{\prime} \subseteq \mathcal{O}\right)$

- $\alpha \in C n\left(\mathcal{O}^{\prime}\right)$ (que implica $\alpha$ )

- Se $\mathcal{O}^{\prime \prime} \subset \mathcal{O}^{\prime} \subseteq \mathcal{O}$, então $\alpha \notin C n\left(\mathcal{O}^{\prime \prime}\right)$ (minimalidade)

Com essas propriedades, concluímos que o conjunto kernel vai conter todas as formas mínimas de se derivar $\alpha$ [Rib13]. Cada elemento do kernel é então chamado de $\alpha$-kernel.

Exemplo 2.1 Considere a base de crenças $\mathcal{O}$, em lógica de descrição:

$$
\begin{aligned}
\mathcal{O}=\{A & \sqsubseteq B, \\
B & \sqsubseteq C, \\
A & \sqsubseteq D, \\
A & \sqsubseteq E, \\
D & \sqcap E \sqsubseteq C\} \models A \sqsubseteq C
\end{aligned}
$$

Nesse caso, construindo o conjunto kernel de $\mathcal{O}$, considerando $\alpha=A \sqsubseteq C$, obtemos:

$$
\begin{aligned}
\mathcal{O} \Perp(A \sqsubseteq C)=\{ & \{A \sqsubseteq B, B \sqsubseteq C\}, \\
& \{A \sqsubseteq D, A \sqsubseteq E, D \sqcap E \sqsubseteq C\}\}
\end{aligned}
$$

Para realizarmos a contração, utilizando a construção kernel, devemos remover elementos de cada um dos elementos contidos no conjunto kernel. Dessa forma, garantimos que as inferências são invalidadas uma a uma, percorrendo todos os subconjuntos de fórmulas a partir dos quais $\alpha$ pode ser inferido. O mecanismo de remoção de elementos do kernel é chamado, na literatura de função de incisão. Essa função é responsável por escolher elementos em cada um dos conjuntos kernel.

Definição 2.2 (Função de Incisão [Han94]) Seja $\mathcal{O}$ uma base de crenças. Para qualquer fórmula $\alpha$, uma função de incisão $\sigma$ para $\mathcal{O}$ é definida como:

- $\sigma(\mathcal{O} \Perp \alpha) \subseteq \bigcup(\mathcal{O} \Perp \alpha) e$

- para todo $\mathcal{K} \in \mathcal{O} \Perp \alpha$, se $\mathcal{K} \neq \emptyset$ então $\mathcal{K} \cap \sigma(\mathcal{O} \Perp \alpha) \neq \emptyset$

Uma boa construção de função de incisão escolheria crenças menos confiáveis para o agente. Assim, a contração kernel pode ser definida como: 
Definição 2.3 (Contração Kernel [Han94]) Seja $\mathcal{O}$ uma base de crenças, $\sigma$ uma função de incisão e a uma fórmula, a contração kernel $-{ }_{\sigma}$ é definida como:

$$
\mathcal{O}-{ }_{\sigma} \alpha=\mathcal{O} \backslash \sigma(\mathcal{O} \Perp \alpha)
$$

O Exemplo 2.2 mostra o funcionamento da contração kernel em conjunto de uma função de incisão.

Exemplo 2.2 Suponha a base de crenças $\mathcal{O}$ :

$$
\begin{aligned}
\mathcal{O}=\{A & \sqsubseteq B, \\
B & \sqsubseteq D, \\
B & \sqsubseteq C, \\
C & \sqsubseteq D\} \models A \sqsubseteq D
\end{aligned}
$$

Considerando $\alpha=A \sqsubseteq D, \mathcal{O} \Perp \alpha$ resulta em:

$$
\begin{aligned}
\mathcal{K}=\mathcal{O} \Perp(A \sqsubseteq D)=\{ & \{A \sqsubseteq B, B \sqsubseteq D\}, \\
& \{A \sqsubseteq B, B \sqsubseteq C, C \sqsubseteq D\}\}
\end{aligned}
$$

Considerando uma função de incisão $\sigma_{\text {freq }}$ que tenta minimizar o número de axiomas removidos, ou seja, removendo primeiro os axiomas que intersectam o maior número de $\alpha$-kernels, como a incisão baseada em frequência proposta por Kalyanpur em [Kal06], obteríamos:

$$
\sigma_{\text {freq }}(\mathcal{K})=\{A \sqsubseteq B\}
$$

Esse é o caso pois o axioma $A \sqsubseteq B$ se encontra em ambos elementos no conjunto kernel. Com isso, o resultado da contração seria:

$$
\begin{aligned}
\mathcal{O}-\sigma_{\text {freq }}(\mathcal{K})=\{B & \sqsubseteq D \\
B & \sqsubseteq C \\
C & \sqsubseteq D\}
\end{aligned}
$$

A troca de função de incisão troca o comportamento da operação de contração, e.g., se utilizarmos uma outra função de incisão que prioriza axiomas levando em conta conectividade sintática de axiomas, o resultado da contração pode ser bem diferente. Essa forma de incisão $\sigma_{\text {syntatic }}$ é chamada de incisão baseada em relevância sintática [Kal06] e funciona calculando uma nota para o axioma sendo analisado baseado no número de axiomas que compartilham das classes em sua assinatura. O axioma escolhido para remoção em cada $\alpha$-kernel é o que possui a menor nota. O Exemplo 2.3 ilustra seu funcionamento:

Exemplo 2.3 (Continuação do Exemplo 2.2) Considerando o conjunto kernel $\mathcal{K}$ apresentado no Exemplo 2.2, ao aplicarmos a função de incisão $\sigma_{\text {syntatic obtemos: }}$ 


$$
\sigma_{\text {syntatic }}(\mathcal{K})=\{A \sqsubseteq B, C \sqsubseteq D\}
$$

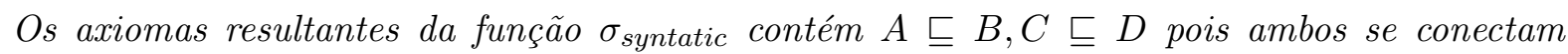
sintaticamente a 2 axiomas, i.e., $A \sqsubseteq B$ compartilha conceitos com $B \sqsubseteq D$ e $B \sqsubseteq C$, os mesmos conectados a $C \sqsubseteq D$. Os axiomas $B \sqsubseteq C$ e $B \sqsubseteq D$ por sua vez estão conectados a 3 outros axiomas cada.

A contração utilizando a função de incisão $\sigma_{\text {syntatic }}$ tem como resultado:

$$
\begin{aligned}
\mathcal{O}-{ }_{\sigma_{\text {syntatic }}}(\mathcal{K})=\{B \sqsubseteq C \\
B \sqsubseteq D\}
\end{aligned}
$$

É fácil notar que essa função não atende ao princípio da minimalidade, uma vez que mais informação do que o necessário foi removido. $\mathrm{O}$ axioma $C \sqsubseteq D$ não precisava ter sido removido, desde que $A \sqsubseteq B$ fosse. Assim, ao utilizar essa função de incisão, devemos nos certificar que o $\alpha$-kernel sendo examinado não possui algum dos elementos que já foram removidos de outros $\alpha$-kernel.

De acordo com o teorema de representação descrito por Hansson e Wassermann [HW02], operadores de contração baseados na contração kernel devem satisfazer os postulados: sucesso, inclusão, uniformidade e core-retainment.

\subsubsection{Contração Partial Meet}

Nessa forma de construção, o critério de mudança mínima é atingido por meio da construção de conjuntos maximais nos quais a inferência $\alpha$ não é válida. $\mathrm{O}$ resultado da contração será portanto, baseado em um conjunto de conjuntos maximais, o que chamaremos de conjunto resíduo - remainder set do inglês [AGM85]. Um conjunto resíduo é definido como:

Definição 2.4 (Conjunto resíduo [AGM85]) Seja a base de crenças $\mathcal{O}$ e a sentença $\alpha$. Um conjunto $\mathcal{O}^{\prime}$ é um elemento pertencente ao conjunto resíduo $\mathcal{O} \perp \alpha$ se e somente se $\mathcal{O}^{\prime}$ é um conjunto maximal de $\mathcal{O}$ que não implica $\alpha$ :

- $\mathcal{O}^{\prime}$ é um subconjunto de $\mathcal{O}\left(\mathcal{O}^{\prime} \subseteq \mathcal{O}\right)$

- $\alpha \notin C n\left(\mathcal{O}^{\prime}\right)$

- se $\mathcal{O}^{\prime} \subset \mathcal{O}^{\prime \prime} \subseteq \mathcal{O}$, então $\mathcal{O}^{\prime \prime} \models \alpha$

Exemplo 2.4 Seja a base de crenças:

$$
\begin{aligned}
\mathcal{O}=\{A & \sqsubseteq B \\
A & \sqsubseteq C \\
& B \sqcap C \sqsubseteq D\} \models A \sqsubseteq D
\end{aligned}
$$


Utilizando $\alpha=A \sqsubseteq D$,obtemos:

$$
\begin{aligned}
\mathcal{O} \perp(A \sqsubseteq D)=\{ & \{A \sqsubseteq B, B \sqcap C \sqsubseteq D\} \\
& \{A \sqsubseteq C, B \sqcap C \sqsubseteq D\} \\
& \{A \sqsubseteq B, A \sqsubseteq C\}\}
\end{aligned}
$$

Como o número de elementos possíveis no conjunto resíduo pode ser grande, fazemos uso de uma função de seleção, responsável por filtrar o conjunto resíduo, selecionando os elementos que contêm as crenças nas quais o agente acredita de forma mais arraigada.

Definição 2.5 (Função de Seleção [AGM85]) Seja $\mathcal{O}$ uma base de crenças. Para qualquer fórmula $\alpha$, uma função de seleção para $\mathcal{O}$ é uma função $\gamma$ t.q., para qualquer fórmula $\alpha$ :

- $S e \mathcal{O} \perp \alpha \neq \emptyset$, então $\gamma(\mathcal{O} \perp \alpha) \neq \emptyset$ e $\gamma(\mathcal{O} \perp \alpha) \subseteq \mathcal{O} \perp \alpha$

- $S e \mathcal{O} \perp \alpha=\emptyset, \gamma(\mathcal{O} \perp \alpha)=\{\mathcal{O}\}$

De modo informal, a contração Partial Meet tem como resultado a interseção da seleção de alguns elementos presentes em $\mathcal{O} \perp \alpha$. Formalmente:

Definição 2.6 (Contração Partial Meet [AGM85])

Sendo $\mathcal{O}$ uma base de crenças, $\alpha$ uma fórmula arbitrária e $\gamma$ uma função de seleção, uma contração do tipo partial meet é definida como:

$$
\mathcal{O}-_{\gamma} \alpha=\bigcap \gamma(\mathcal{O} \perp \alpha)
$$

Exemplo 2.5 Seja a base de crenças:

$$
\begin{aligned}
\mathcal{O}=\{A & \sqsubseteq B \\
B & \sqsubseteq C \\
C & \sqsubseteq D\} \models A \sqsubseteq D
\end{aligned}
$$

Ao construirmos o conjunto resíduo, utilizando $\alpha=A \sqsubseteq D$ obtemos:

$$
\begin{aligned}
\mathcal{O} \perp(A \sqsubseteq D)=\{ & \{A \sqsubseteq B, B \sqsubseteq C\}, \\
& \{A \sqsubseteq B, C \sqsubseteq D\}, \\
& \{B \sqsubseteq C, C \sqsubseteq D\}\}
\end{aligned}
$$

Um operador de contração Partial Meet que utilizasse um função de seleção $\gamma_{\text {syntatic }}$ que seleciona elementos seguindo o mesmo paradigma proposto por Kalyanpur em [Kal06], apresentado no exemplo 2.3 selecionaria elementos do conjunto resíduo que possuíssem o maior nível de conexão sintática entre seus elementos. O resultado da seleção seria:

$$
\begin{aligned}
\mathcal{O}-\gamma_{\text {syntatic }}= & \{\{A \sqsubseteq B, B \sqsubseteq C\}, \\
& \{B \sqsubseteq C, C \sqsubseteq D\}\}
\end{aligned}
$$


A escolha foi feita pois ambos axiomas nos dois elementos do conjunto resíduo estão conectados sintaticamente, ao passo que no elemento $\{A \sqsubseteq B, C \sqsubseteq D\}$, os axiomas não compartilham conceitos entre si.

Segundo o teorema de representação de Hansson, em [HW02], uma construção do tipo Partial Meet tem de estar de acordo com os postulados: sucesso, inclusão, relevância e uniformidade.

\subsection{Construção de Conjuntos Kernel}

A construção de conjuntos minimalmente inconsistentes é um problema bastante estudado na literatura e é de fundamental importância para a construção de serviços para depuração de ontologias bem como serviços para a revisão de crenças. Esses conjuntos podem trazer à atenção do projetista da ontologia o trecho da mesma que apresenta problemas em suas definições.

Ao longo dos últimos anos várias ferramentas para a depuração de ontologias surgiram, basicamente advindas do meio acadêmico. É o caso do Swoop [KPH05][Kal06], do plugin RaDon para o Neon Toolkit [JHQ ${ }^{+}$09], do explanation workbench presente no Protégé 4 [HPS08] OWLExplanation plugin ${ }^{1}$.

Todas essas ferramentas tem como objetivo apresentar ao usuário explicações mínimas para uma determinada inferência, podendo ser utilizadas para gerar explicações para incoerências, inconsistências e inferências normais. Entretanto, apesar dos esforços para construção de tais ferramentas, ainda é um desafio o projeto de uma ferramenta que possa ser útil e intuitiva para engenheiros de ontologias.

O cálculo desse tipo de subconjunto também está no núcleo nos trabalhos que dependem de conjuntos minimais que justifiquem um determinado axioma e que buscam a construção de justificativas como Kalyanpur [Kal06] e Horridge [Hor11]. Também é componente importante para trabalhos na área de revisão de crenças como [HWKP06], [Was00].

\subsubsection{Algoritmos para Cálculo de Um Único Elemento do Kernel}

Nesta Seção descreveremos uma classe de algoritmos, chamados de Black-box para a construção de um único elemento do conjunto Kernel. Estes algoritmos recebem esse nome pois são capazes de construir um conjunto kernel utilizando um mecanismo de inferência já existente, sem realizar modificações. Em contraposição aos algoritmos Black-box estão os algoritmos Glass-box. Estes algoritmos se baseiam em modificações feitas internamente ao mecanismo de inferência, o que resulta em um ganho de desempenho, porém um aumento na complexidade de implementação.

O algoritmo Black-box para cálculo de kernel se baseia num princípio de expande-encolhe, onde o algoritmo inicia com um conjunto vazio e adiciona axiomas até ser possível realizar a inferência indesejável. Após essa fase temos a garantia de que o conjunto construído, ou algum sub-conjunto realiza a inferência indesejável.

A segunda fase faz o encolhimento do conjunto obtido, i.e., tenta remover, deste conjunto, qualquer axioma que não contribui com a inferência indesejada.

Chamadas ao mecanismo de inferências se fazem necessárias a cada passo da expansão, para garantir que, a partir do conjunto sendo construído, seja possível realizar a inferência indesejada.

\footnotetext{
${ }^{1}$ https://github.com/matthewhorridge/owlexplanation
} 
De forma análoga, durante o encolhimento, o mecanismo de inferências também é utilizado a cada passo, para verificar que o axioma que está sendo removido do conjunto conflito não é relevante para a inferência indesejável.

O algoritmo Black-box pode ser visto na Listagem 2.1

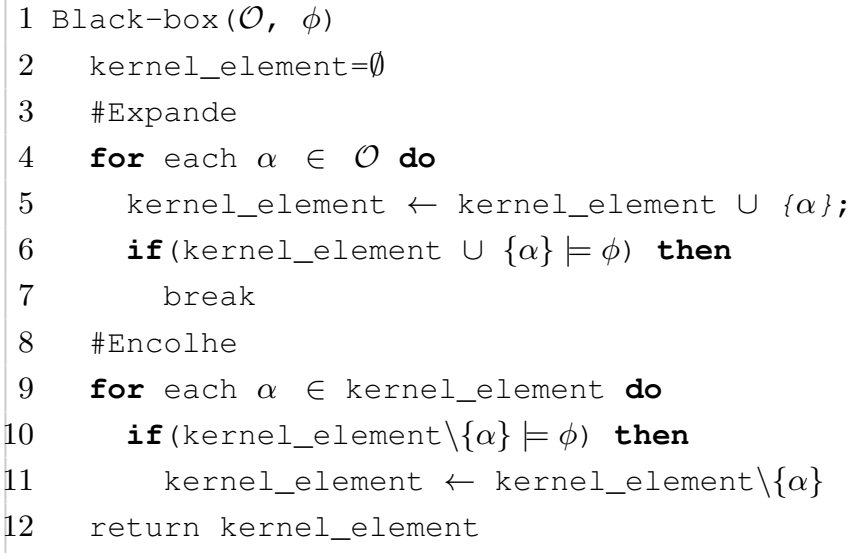

Listagem 2.1: Algoritmo Black-box

O algoritmo descrito parte de um $\alpha$-kernel vazio, porém uma outra forma de implementá-lo é evitar a fase de expansão, iniciando o conjunto kernel_element com a ontologia $\mathcal{O}$. Dessa forma, as chamadas ao mecanismo de inferências, durante a fase de expansão, são evitadas. Em uma análise de pior caso, o número de chamadas é reduzido. Porém, de acordo com nossos experimentos (Seção 4.4), não é comum que ontologias inteiras estejam envolvidas em uma inferência indesejada. É comum que tais conjuntos representem uma fração pequena do tamanho da ontologia, o que nos leva a crer que, em uma análise de caso médio, ambos algoritmos apresentem desempenhos similares. Chamaremos a técnica de adição direta do conjunto inteiro, durante a expansão/encolhimento de expansão/encolhimento trivial.

O desempenho de ambas implementações são características impeditivas para suas adoções práticas. Em [Sun09], Suntisrivaraporn mostra que esse tipo de implementação não pode ser aplicada para a construção de elementos kernel em ontologias de grande porte, como a SNOMED.

Pensando na complexidade desses algoritmos, algumas otimizações foram propostas com o objetivo de minimizar o número de chamadas ao mecanismo de inferências tanto durante a fase de expansão quanto durante o encolhimento, minimizando assim o tempo de execução. A aplicação de tais técnicas de otimização tentam construir, de forma mais eficiente possível um conjunto onde a inferência indesejável possa ser feita durante a fase de expansão ou remover o maior número de axiomas do conjunto, de forma a atingir o critério de minimalidade o mais rápido possível.

\subsubsection{Otimizações durante a Fase de Expansão}

Otimizações nessa fase buscam essencialmente construir um conjunto de conflitos que seja o mais próximo do resultado minimal, com o menor número de interações.

A forma mais comum desse tipo de otimização é via função de seleção, que tem como objetivo, escolher elementos que estejam envolvidos na inferência indesejada de acordo com algum critério de relevância.

A ideia de função de seleção se assemelha à estratégia apresentada por Haase et al. em [HvHH $\left.{ }^{+} 05\right]$. Nesse trabalho os autores propõem um algoritmo para a construção de kernels, que, partindo de 
um conceito não satisfazível, cria um conjunto minimal que explique o porquê da insatisfazibilidade do conceito. Axiomas são escolhidos para a inserção nesse conjunto por meio de uma função de seleção chamada de conectividade estrutural (structural connectedness).

A noção de conectividade estrutural ou sintática adiciona elementos ao conjunto kernel de acordo com a sua proximidade sintática do conceito insatisfazível, i.e., axiomas que possuem o conceito em questão em suas assinaturas. A mesma noção de conectividade estrutural foi utilizada em [SQJH08, KPHS07, JQH09]. O seguinte exemplo ilustra o funcionamento da conectividade estrutural.

Considere a seguinte ontologia:

$$
\mathcal{O}=\{A \sqsubseteq B, B \sqsubseteq C, A \sqsubseteq \neg C, D \sqsubseteq E, E \sqsubseteq A, D \sqsubseteq F\}
$$

É fácil de ver que o conceito $A$ não possui elementos em sua interpretação, ou seja, é um conceito necessariamente vazio, i.e., $A \sqsubseteq \perp$.

Utilizando a noção de conectividade estrutural, para a depuração dessa ontologia partimos do conceito insatisfazível $A$ e criamos uma nova ontologia com os axiomas que estão próximos sintaticamente a $A$ :

$$
\mathcal{O}^{\prime}=\{A \sqsubseteq B, A \sqsubseteq \neg C, E \sqsubseteq A\}
$$

A partir de $\mathcal{O}^{\prime}$, não podemos derivar a inferência indesejada. Nesse momento, em uma segunda iteração da função de seleção, adicionamos a $\mathcal{O}^{\prime}$ todos os axiomas cujas assinaturas possuem classes em comum com as assinaturas dos axiomas em $\mathcal{O}^{\prime}$ i.e., todos os axiomas que possuírem $A, B, C$ e $E$ em suas assinaturas. $\mathrm{O}$ que resulta na expansão de $\mathcal{O}^{\prime}$ para $\mathcal{O}^{\prime \prime}$ abaixo onde $\mathcal{O}^{\prime \prime} \models A \sqsubseteq \perp$ :

$$
\mathcal{O}^{\prime \prime}=\{A \sqsubseteq B, B \sqsubseteq C, A \sqsubseteq \neg C, D \sqsubseteq E, E \sqsubseteq A\}
$$

Apesar de acelerar o processo de encontrar um conjunto no qual a inferência indesejada seja válida, é fácil ver que esse tipo de função de seleção, em poucas iterações cresce de maneira rápida. No nosso exemplo, em apenas 3 iterações teríamos a nossa ontologia $\mathcal{O}$ inteira. Huang et al. [HvHtT05] apresentam uma melhoria para a função de conectividade estrutural chamada relevância de conceito, onde a função de relevância somente considera dois axiomas $\phi$ e $\psi$ se e somente se

1. Se $\phi$ for da forma $C \sqsubseteq D$, então $C$ pertence a assinatura de $\psi$;

2. Se $\phi$ for da forma $C \equiv D$, então $C$ ou $D$ pertencem a assinatura de $\psi$;

3. Se $\phi$ for da forma $C \sqsubseteq \neg D$, então $C$ ou $D$ pertencem a assinatura de $\psi$;

O algoritmo para a função de conectividade estrutural utilizando relevância de conceitos pode ser visto na Listagem 2.3. Esse algoritmo faz uso da rotina apresentada na Listagem 2.2, que é responsável por estabelecer a noção de conectividade estrutural definida por Huang et al. [HvHtT05] entre dois axiomas

1 Structurally_Connected? $(\phi, \psi)$

2 if ( $\phi$ of the form $C \sqsubseteq D$ ) then

3 if $C \in \operatorname{Sig}(\psi)$ then 


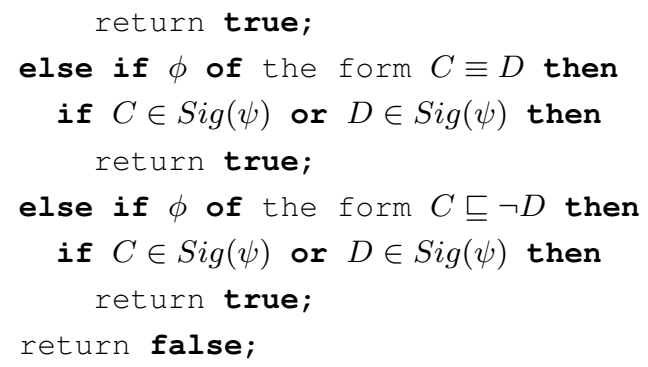

Listagem 2.2: Algoritmo para cálculo de conectividade estrutural entre dois axiomas

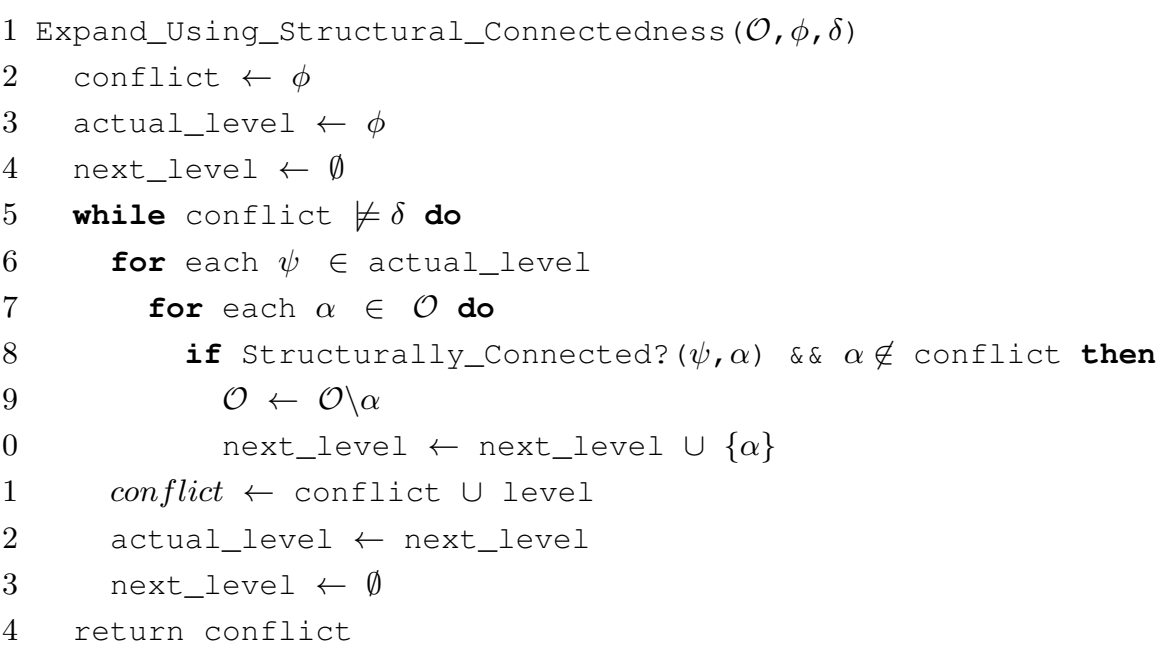

Listagem 2.3: Algoritmo para cálculo de conectividade estrutural

Se utilizarmos a noção de relevância de conceito e retornarmos ao nosso exemplo, veremos que na primeira iteração, obtemos:

$$
\mathcal{O}^{\prime}=\{A \sqsubseteq B, A \sqsubseteq \neg C\}
$$

Após uma segunda interação obtemos o conjunto:

$$
\mathcal{O}^{\prime \prime}=\{A \sqsubseteq B, A \sqsubseteq \neg C, B \sqsubseteq C\}
$$

$\mathcal{O}^{\prime \prime}$ é um conjunto minimal, portanto, nesse caso, a fase de encolhimento não alteraria o conjunto. Infelizmente a fase de encolhimento é necessária pois não podemos estabelecer se algum dos axiomas no conjunto pode ser descartado.

Embora no pior caso o algoritmo proposto por Huang et al. em [HvHtT05] também expanda o conjunto conflito até a ontologia completa rapidamente, os autores realizaram experimentos com essa abordagem em ontologias do mundo real e obtiveram resultados que mostram que essa abordagem é mais eficiente que a função de seleção não otimizada.

Uma outra forma de pensarmos na otimização da fase de expansão é dividir a ontologia em compartimentos. Essa ideia segue a mesma motivação de trabalhos na área de Revisão de crenças feitas por agentes que possuem restrições [Was00], ou seja não são máquinas ideais. As restrições em questão são a respeito da memória e da capacidade de inferência. Esse mesmo tipo de problemática se aplica ao desenvolvimento de ontologias.

$\mathrm{Na}$ área de revisão de crenças com recursos limitados, Wassermann define em [Was00], operadores para revisão de crenças que realizam operações de forma local, i.e., realizando alterações 
somente em partes consideradas relevantes.

Ao criar compartimentos ou módulos [VPSS10] otimizamos o uso do mecanismo de inferências, que tem que realizar processamento em conjuntos menores de axiomas. Um outro efeito colateral é um melhor aproveitamento da memória, uma vez que não precisamos ter a ontologia inteira em memória para processamento. Horridge [Hor11] cita ainda os benefícios de inclusão de um limite superior para funções de seleção - lidando com módulos garantimos que funções de seleção não eficientes não vão adicionar todos os elementos da ontologia ao conjunto conflito.

\subsubsection{Otimizações durante a Fase de Encolhimento}

Otimizações para essa fase do algoritmo Black-box concentram-se em formas de minimizar o conjunto conflito encontrado pela primeira fase de forma mais rápida e com o menor número de chamadas ao mecanismo de inferências possível.

\subsubsection{Janelas Deslizantes}

Para lidar com esse problema, Kalyanpur et al. [Kal06, KPHS07] propôs um esquema de janelas deslizantes que tenta remover, no lugar de um único axioma, vários axiomas de uma única vez, criando a ideia de um quadro, ou janela, que desliza por sobre o conjunto conflito, uma fórmula por vez. Quando essa janela encontra um conjunto de fórmulas que pode ser removido, sem que a inferência indesejada deixe de existir, ele o remove. Por exemplo, imagine que o algoritmo Blackbox da Listagem 2.1 retorna o seguinte conjunto conflito e que o algoritmo é executado com um tamanho de janela $=3$ :

$$
\mathcal{O}=\{A \sqsubseteq B, B \sqsubseteq C, A \sqsubseteq \neg C, D \sqsubseteq E, E \sqsubseteq A, D \sqsubseteq F\}
$$

Na primeira iteração o algoritmo seleciona os três primeiros axiomas e tenta removê-los i.e.:

$$
\mathcal{O}=\{A \sqsubseteq B, B \sqsubseteq C, A \sqsubseteq \neg C, D \sqsubseteq E, E \sqsubseteq A, D \sqsubseteq F\}
$$

Porém, a remoção desse conjunto de axiomas faz com que a inferência indesejada $(A \sqsubseteq \perp)$ seja invalidada também. Assim, o algoritmo segue por mais 2 iterações, i.e.:

$$
\begin{aligned}
& \mathcal{O}=\{A \sqsubseteq B, B \sqsubseteq C, A \sqsubseteq \neg C, D \sqsubseteq E, E \sqsubseteq A, D \sqsubseteq F\} \\
& \mathcal{O}=\{A \sqsubseteq B, B \sqsubseteq C, A \sqsubseteq \neg C, D \sqsubseteq E, E \sqsubseteq A, D \sqsubseteq F\}
\end{aligned}
$$

Em ambos os casos, a remoção não é bem sucedida. Durante a terceira iteração o algoritmo seleciona os três últimos axiomas, que podem ser removidos de forma não prejudicial a inferência indesejada, i.e. 


$$
\mathcal{O}=\{A \sqsubseteq B, B \sqsubseteq C, A \sqsubseteq \neg C, D \sqsubseteq E, E \sqsubseteq A, D \sqsubseteq F\}
$$

Desta forma, o número de chamadas ao mecanismo de inferências é reduzido, otimizando o tempo de execução do algoritmo Black-box. A descrição do algoritmo de janelas deslizantes se encontra na Listagem 2.4.

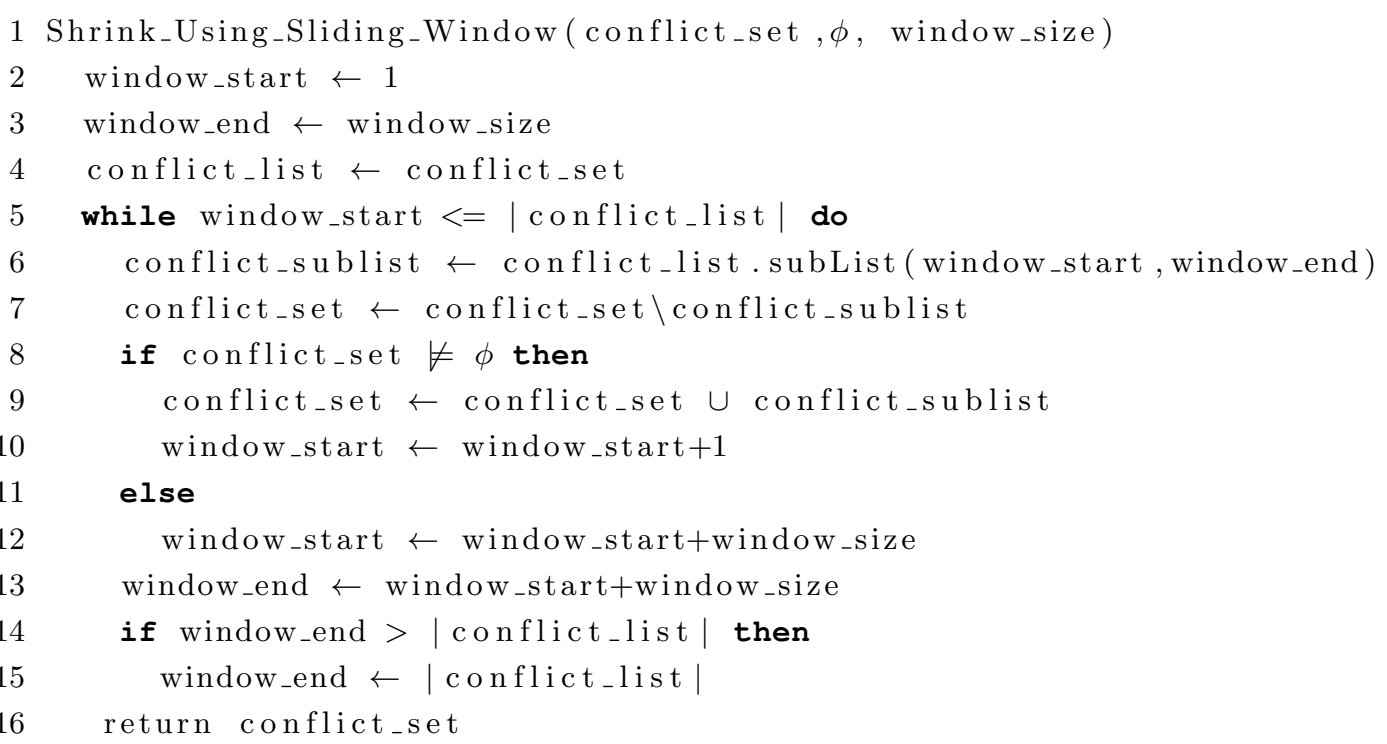

Listagem 2.4: Algoritmo Janelas Deslizantes [Kal06]

\subsubsection{Algoritmo divisão e conquista}

A minimização do uso do mecanismo de inferências pode ser obtida utilizando um mecanismo baseado em divisão e conquista como proposto por Junker em [Jun01]. A estratégia basicamente consiste em dividir o conjunto conflito $\mathcal{K}$ em dois sub-conjuntos $\mathcal{K}_{1}$ e $\mathcal{K}_{2}$ menores e em seguida verificar se, a partir de algum deles, a inferência indesejada pode ser realizada. Se esse for o caso, então conseguimos descartar metade dos axiomas no conjunto conflito original. Essa tarefa é executada recursivamente na metade capaz de realizar a inferência indesejada, até atingirmos um conjunto conflito minimal.

Caso nem $\mathcal{K}_{1}$ nem $\mathcal{K}_{2}$ possa realizar a inferência indesejada dividimos um deles, e.g., $\mathcal{K}_{1} \mathrm{em}$ $\mathcal{K}_{11}$ e $\mathcal{K}_{12}$ e verificamos se a união de um deles a $\mathcal{K}_{2}$ pode realizar a inferência indesejável.

O algoritmo para essa abordagem pode ser visto na Listagem 4.3.

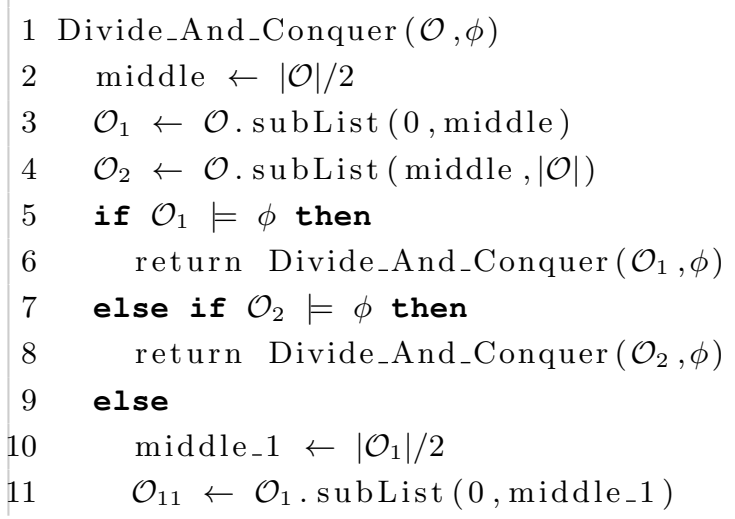




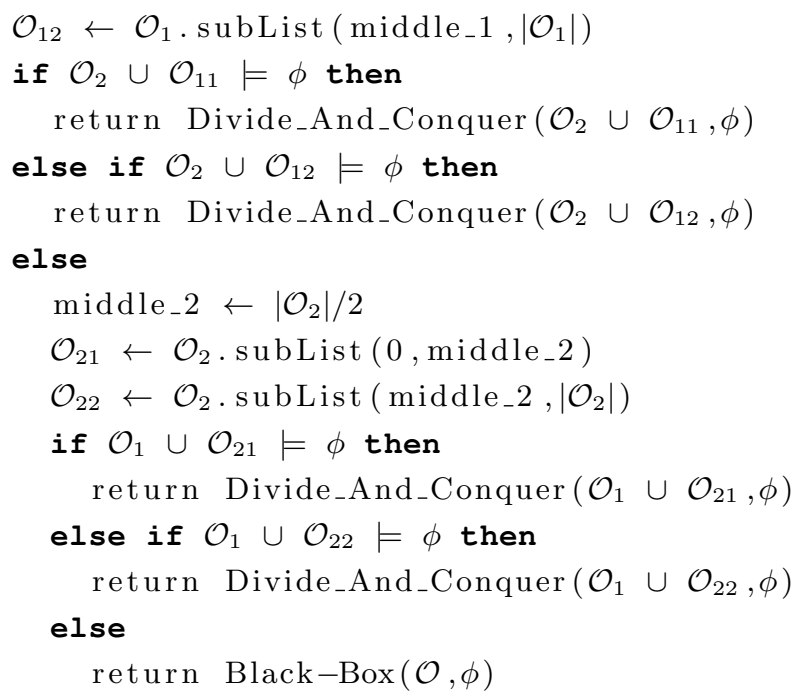

Listagem 2.5: Algoritmo Divisão e Conquista

Ao aplicarmos o algoritmo na Listagem 4.3 com o nosso exemplo anterior obtemos,

$$
\begin{aligned}
& \mathcal{O}_{1}=\{A \sqsubseteq B, B \sqsubseteq C, A \sqsubseteq \neg C\} \\
& \mathcal{O}_{2}=\{D \sqsubseteq E, E \sqsubseteq A, D \sqsubseteq F\}
\end{aligned}
$$

Percebemos que, a partir de $\mathcal{O}_{1}$ podemos realizar a inferência indesejada $(A \sqsubseteq \perp)$, então descartamos metade de $\mathcal{O}$ na primeira iteração.

Realizamos comparação entre as otimizações janelas deslizantes e divisão e conquista. Os dados dessa comparação podem ser vistos na Seção 4.4. Confirmamos os resultados levantado por Shchekotykhin et al. em [SFJ08], em um conjunto de experimentos com ontologias do mundo real. Em nossos experimentos comprovamos que a abordagem divisão e conquista realiza um número menor de chamadas ao mecanismo de inferências, resultando em um menor tempo de execução.

\subsubsection{Algoritmos para Cálculo do Conjunto Kernel Completo}

Embora Horridge [Hor11] afirme que, encontrar um único elemento do conjunto kernel pode ser útil para o engenheiro de ontologias, pois os problemas apontados pelo kernel chamam a atenção do engenheiro para trechos com defeito de modelagem; acreditamos que, para o caso da fusão, a construção de um único elemento do conjunto não é de grande ajuda.

Para encontrar os demais elementos do conjunto kernel, Kalyanpur [Kal06] e Wassermann [Was00] utilizam uma modificação do algoritmo de Reiter [Rei87] para cálculo de cortes mínimos para diagnóstico de sistemas defeituosos.

A área de diagnóstico é bem ativa dentro da Inteligência Artificial, e busca resolver o seguinte problema: dada uma observação de um comportamento anormal em um sistema, isolar quais componentes podem estar defeituosos, causando tal comportamento [Rei87]. A resolução desse tipo de problema deu origem ao que hoje é conhecido como diagnóstico baseado em modelos [HCdK92], onde os modelos de sistemas são dados em alguma linguagem formal.

Um diagnóstico para um sistema é composto de um conjunto minimal de componentes que, se substituídos, fazem com que o comportamento indesejado seja reparado. Esses componentes são 
retirados de conjuntos, chamados conjuntos conflito que contêm elementos pertencentes ao sistema, cuja interação pode gerar o comportamento anormal [Hor11].

Para obter um diagnóstico a partir de um conjunto de conjuntos conflito utilizamos o algoritmo Reiter para cálculo de cortes mínimos (Hitting Sets). O objetivo do algoritmo para cálculo de cortes mínimos é selecionar pelo menos um dos elementos em cada conjunto conflito, reparando o comportamento anômalo do sistema.

Definição 2.7 (Corte Mínimo) Um corte em uma classe de conjuntos $\mathcal{C}$ é um conjunto $B$ de modo que, para qualquer $C \in \mathcal{C}, B \cap C \neq \emptyset$. Esse corte é considerado minimo se e somente se não existe um $B^{\prime}$ tal que, $B^{\prime}$ é um corte na classe $\mathcal{C}$ e $B^{\prime} \subset B$.

A ligação entre o cálculo de diagnósticos e revisão de crenças foi estabelecida por Wassermann, em [Was00], onde um diagnóstico correspondia a um conjunto de cortes mínimos. Essa noção foi aumentada por Kalyanpur em [Kal06] para depuração de ontologias e construção de reparos para ontologias. Dessa forma, um comportamento anormal é visto como uma inferência indesejada, um conjunto conflito minimal é visto como um kernel. Um diagnóstico é visto como a definição de um plano de reparo para o conjunto kernel completo.

Horridge em [Hor11], mostrou que o algoritmo pode ser utilizado para a construção de justificativas mínimas para uma determinada inferência. Como o problema de depuração pós fusão se assemelha em muitos aspectos com as tarefas de depuração, cálculo de justificativas e revisão de crenças, acreditamos que o desafio de encontrar conjuntos conflito mínimos também é de extrema pertinência para a depuração pós fusão.

O Algoritmo Hitting Set Tree mostrado na Listagem 2.6 calcula uma estrutura de árvore, na qual os nós possuem conjuntos conflitos minimais e as arestas possuem axiomas pertencentes a ontologia sendo depurada. O caminho que une as folhas da árvore a raiz é um diagnóstico ou um reparo para a ontologia.

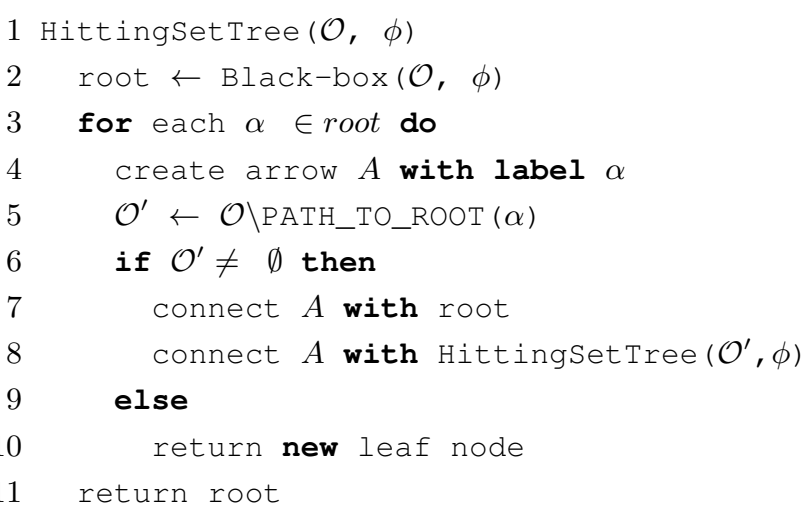

Listagem 2.6: Algoritmo Hitting Set Tree

O algoritmo listado cria a árvore de cortes mínimos e a intuição por trás do algoritmo é: primeiramente construímos um conjunto de conflitos para ser armazenado no nó raiz. Em seguida, iteramos sobre esse conjunto, removendo individualmente, a cada iteração, um axioma $\alpha$. Criamos uma aresta com o rótulo $\alpha$ removido da vez. Esse passo corresponde a calcular um dos elementos do plano de reparo da ontologia. Em seguida, conectamos essa aresta ao nó resultante da execução recursiva do algoritmo, tendo como entrada a nova base (após remoção da fórmula $\alpha$ ) e a inferência indesejada $\phi$. O funcionamento do algoritmo é apresentado no exemplo 2.6. 
Exemplo 2.6 Suponha que tenhamos a seguinte ontologia:

$$
\begin{aligned}
\mathcal{O}=\{A & \sqsubseteq B \\
B & \sqsubseteq D \\
A & \sqsubseteq E \\
E & \sqsubseteq D\}
\end{aligned}
$$

$A$ partir de $\mathcal{O}$ podemos inferir que $A \sqsubseteq D$. Porém é importante notar que podemos fazer isso via duas justificativas: $\mathcal{J}_{1}=\{A \sqsubseteq B, B \sqsubseteq D\}$ e $\mathcal{J}_{2}=\{A \sqsubseteq E, E \sqsubseteq D\}$. Ao executarmos o algoritmo apresentado na Listagem 2.6 construímos a seguinte árvore.

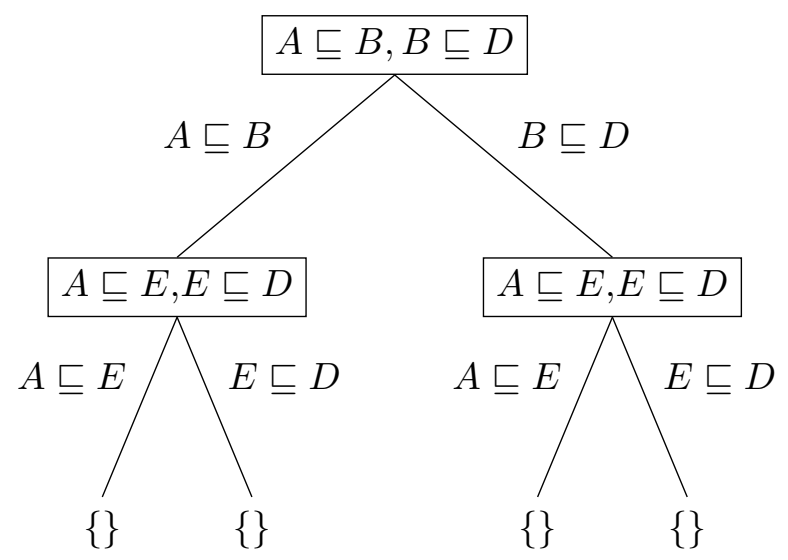

Figura 2.1: Árvore de cortes mínimos - Exemplo 2.6.

No primeiro passo do algoritmo, a raiz da árvore é construída com uma chamada ao algoritmo Black-box. Essa chamada resulta no conjunto conflito $\mathcal{K}_{1}=\{A \sqsubseteq B, B \sqsubseteq D\}$. Na linha 3, temos um laço que remove a cada iteração um axioma do conjunto $\mathcal{K}_{1}$. Na primeira iteração, construímos $\mathcal{O}^{\prime}=\{B \sqsubseteq D, A \sqsubseteq E, E \sqsubseteq D\}$ e novamente executamos o Black-box, obtendo o conjunto $\mathcal{K}_{2}=$ $\{A \sqsubseteq E, E \sqsubseteq D\}$, que ocupará o nó mais a esquerda da árvore no primeiro nível. Do lado esquerdo da árvore, construímos $\mathcal{O}^{\prime}=\{A \sqsubseteq B, A \sqsubseteq E, E \sqsubseteq D\}$ e após a execução do Black-box preenchemos o nó mais a direita do primeiro nível da nossa árvore com o conjunto $\mathcal{K}_{3}=\mathcal{K}_{2}=\{A \sqsubseteq E, E \sqsubseteq D\}$. A partir desse nível não podemos mais gerar conjuntos conflitos utilizando o Black-box.

\subsubsection{Otimizações ao Algoritmo de Cálculo de Árvore de Cortes Mínimos}

É fácil de ver que o gargalo para o algoritmo de construção de Árvores de cortes mínimos é o número de chamadas à rotina Black-box. Nas próximas seções listaremos duas abordagens cuja adoção impacta positivamente no tempo de execução.

\subsubsection{Fechamento Precoce de Caminhos}

Um nó na árvore de cortes mínimos pode ser considerado um nó aberto, a partir do qual construiremos sub-árvores, ou um nó fechado, como as folhas que não podem ser expandidas. A capacidade de detecção de tal condição é uma otimização chave para o algoritmo de cortes mínimos. A principal condição detectada para o fechamento precoce de um caminho é o nó aberto $n_{1}$ possuir 
um conjunto rotulado de nós até a raiz igual ao de um nó $n_{2}$. Sendo esse o caso, podemos deixar de calcular os sucessores de $n_{2}$, pois serão os mesmos de $n_{1}$.

Conseguimos realizar esse fechamento precoce devido ao fato que o cálculo do rótulo para o nó "sucessor" é feito a partir de uma cópia da ontologia de entrada removidos todos os axiomas que estão no caminho do nó até a raiz.

Exemplo 2.7 Suponha que temos a seguinte ontologia:

$$
\begin{aligned}
\mathcal{O}= & \{A \sqsubseteq B \\
& A \sqsubseteq \neg B \\
& A \sqsubseteq C \\
& C \sqsubseteq B \\
& B \sqsubseteq D \\
& A \sqsubseteq \neg D \\
& A \sqsubseteq E \\
& A \sqsubseteq \neg E\}
\end{aligned}
$$

É fácil notar que de $\mathcal{O}$ conseguimos inferir que $A \sqsubseteq \perp$. Utilizando o algoritmo de cálculo da árvore de cortes mínimos mostrado na Listagem 2.6, o resultado das primeiras iterações podem ser vistos na Figura 2.2.

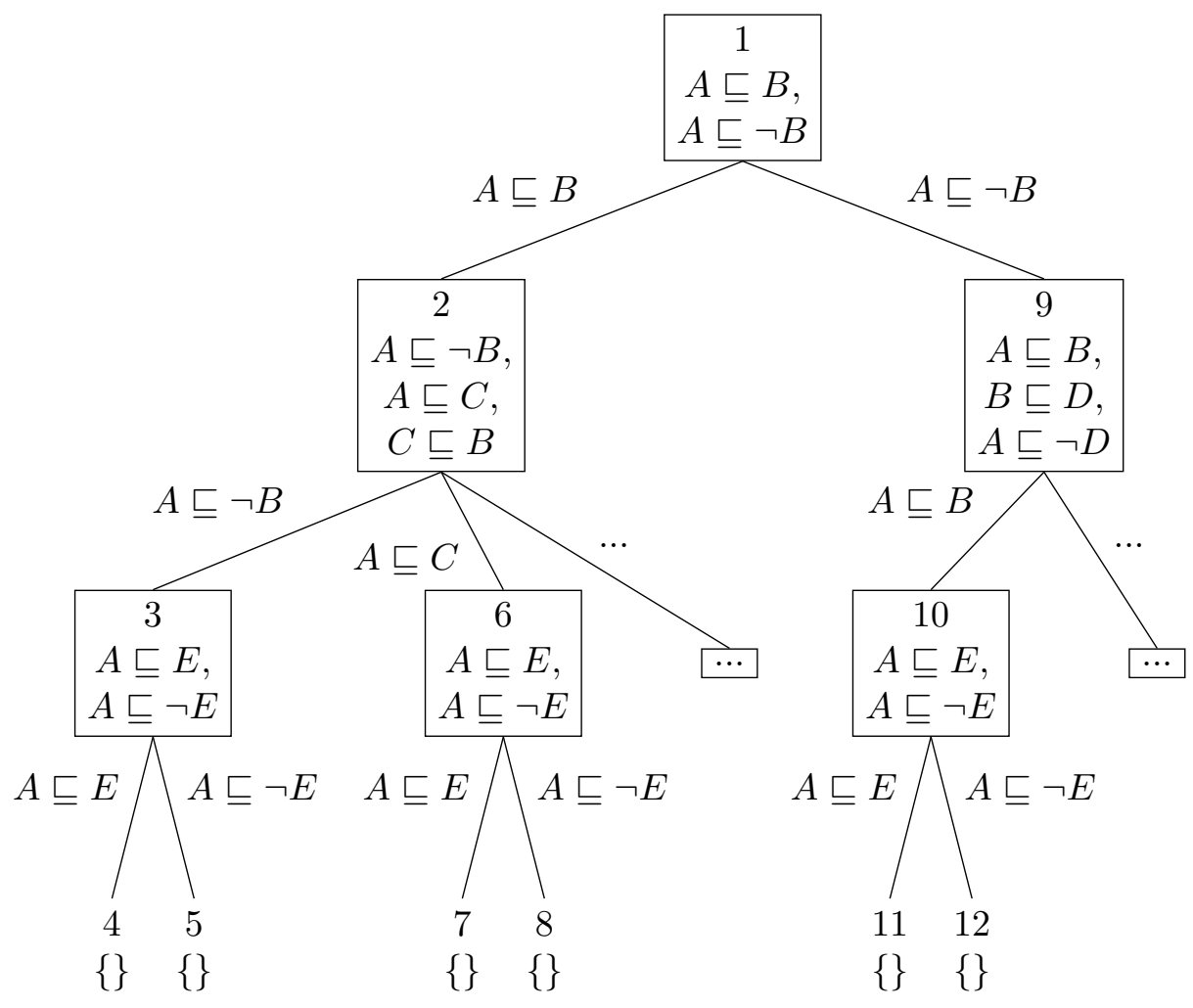

Figura 2.2: Árvore de cortes mínimos - Exemplo 2.7.

A expansão ocorre de forma normal, porém, ao expandirmos o nó 10, utilizando o axioma $A \sqsubseteq B$, percebemos que seu caminho até a raíz é o mesmo do nó 3, i.e. $P=\{A \sqsubseteq B, A \sqsubseteq \neg B\}$. Utilizando a otimização de fechamento precoce de caminho não precisaríamos realizar a expansão 
desse nó. Iremos representar um fechamento de caminho utilizando uma marcação $\times$ no nó. Assim, o resultado da utilização da otimização pode ser visto na Figura 2.3.

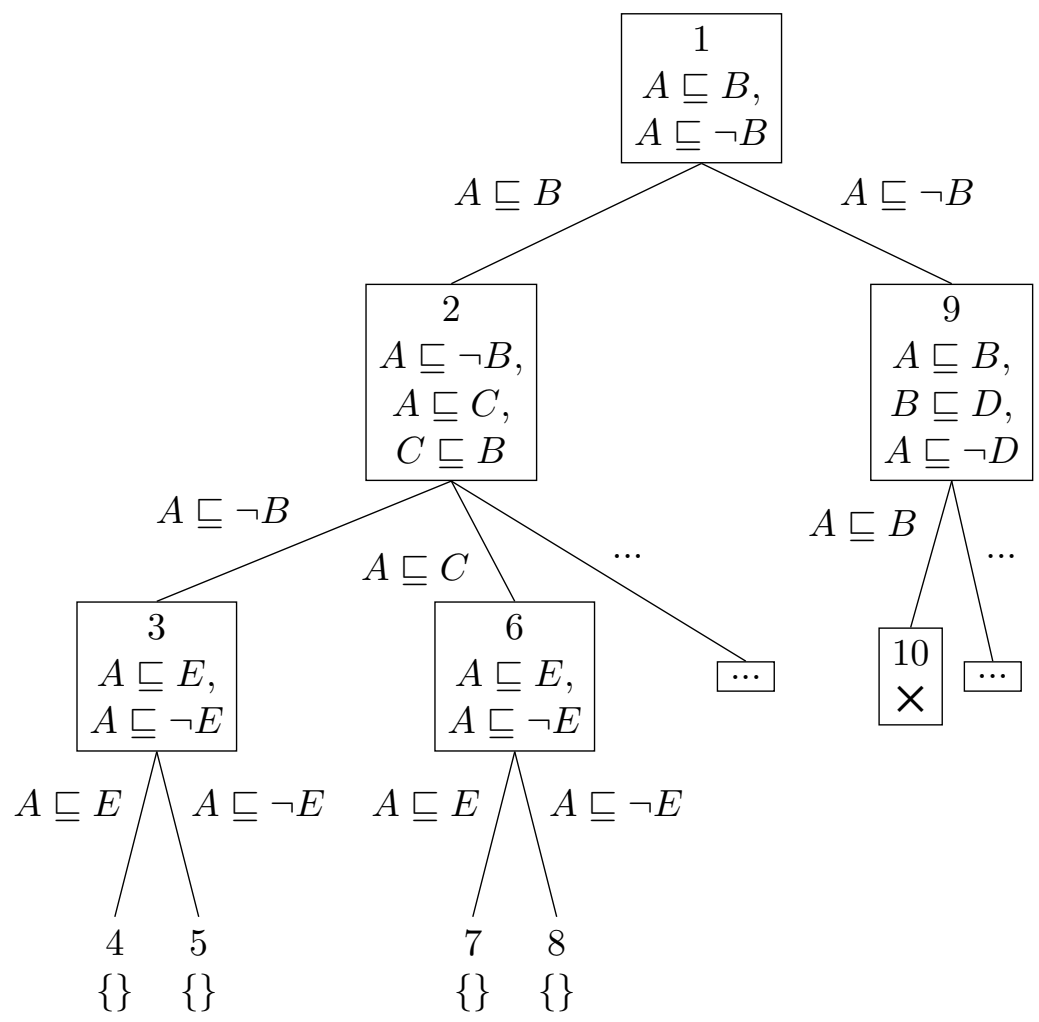

Figura 2.3: Árvore de cortes mínimos - Fechamento do nó 10.

Podemos ver que a adoção da otimização faz com que evitemos uma nova chamada ao procedimento black-box, poupando assim várias chamadas ao mecanismo de inferências.

\subsubsection{Reutilização de Nós}

Nessa otimização, buscamos realizar a redução do número de chamadas ao Black-box por meio do reuso de nós já calculados. Partindo do princípio que o rótulo de um novo nó é calculado a partir da exclusão dos axiomas descritos no caminho desse nó até a raiz da ontologia, procuramos então um nó cujo rótulo não intersecte com o caminho até a raiz do nó atual. Existindo esse nó, não mais precisamos encontrar um novo conjunto conflito disjunto dos elementos listados no caminho desse nó até a raiz. Com essa otimização, a árvore pode ser vista como um grafo acíclico dirigido. Dessa forma os nós não são duplicados. Fazemos com que a expansão aponte para um nó já "aberto".

O funcionamento dessa otimização é mostrado no Exemplo 2.8 .

Exemplo 2.8 Considere a ontologia $\mathcal{O}$ apresentada no Exemplo 2.7 e a árvore de cortes mínimos mostrada na Figura 2.2. Nessa expansão, os nós 6 e 10 realizam chamadas ao procedimento blackbox para construir um conjunto conflito, cuja interseção com o seu caminho até a raiz seja vazio. Entretanto, o nó 3 já realizou a construção desse conjunto, i.e., $\mathcal{K}=\{A \sqsubseteq E, A \sqsubseteq \neg E\}$, que também possui interseção vazia com os caminhos até a raiz dos nós 6 e 10. Utilizaremos a marcação para representar o reuso de um nó.

Dessa forma, a árvore de caminhos mínimos para a ontologia $\mathcal{O}$ apresentada no Exemplo 2.7 pode ser vista na Figura 2.4. 


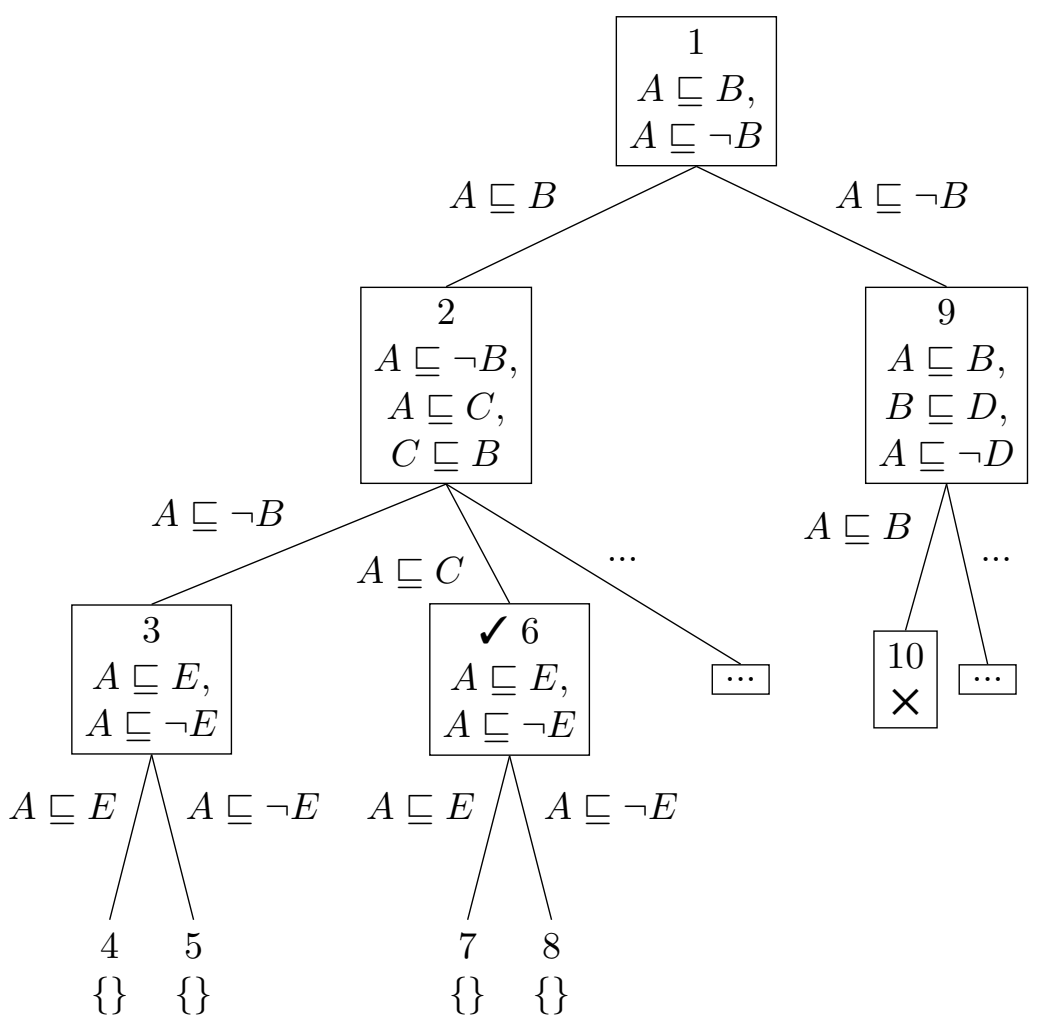

Figura 2.4: Árvore de cortes mínimos - Fechamento do nó 10 e reuso do nó 6.

\subsection{Construção de Conjuntos Resíduos}

A construção dos chamados conjuntos resíduos é de fundamental importância para a depuração de ontologias. Em especial em casos onde o projetista não deseja repassar todas as possíveis causas de uma inferência indesejada. Também é bastante utilizada na contração de crenças (belief contraction) como visto anteriormente.

$\mathrm{Na}$ área de revisão de ontologias, alguns trabalhos como os de Flouris[Flo06] e Ribeiro [Rib13] realizam caracterizações dessas operações, porém nenhuma construção é mostrada.

Em [RW08], Ribeiro e Wassermann mostram que existem formas de realizar a construção de conjuntos resíduos a partir do conjunto kernel. Bastando, para tal, apenas utilizar uma função de incisão minimal. Eles mostraram que tal função pode ser obtida utilizando o algoritmo de árvore de cortes mínimos mostrado na Seção 2.2.4.

O problema com essa abordagem é a necessidade de construir o conjunto kernel inteiro para, a partir dele, gerar o conjunto resíduo. Essa construção pode ser bastante custosa. A abordagem para cálculo da árvore de cortes mínimos realiza chamadas a um algoritmo que calcula itens no conjunto kernel. Tipicamente, em uma implementação baseada em Black-box, várias chamadas ao mecanismo de inferência são realizadas - em lógicas do tipo $\mathcal{A L C}$ essas chamadas possuem complexidade PSPACE-COMPLETO $\left[\mathrm{BCM}^{+} 03\right]$. Nesse trabalho, testamos a hipótese de que a construção de conjuntos resíduos pode diminuir o número de chamadas a esses mecanismos no Capítulo 4.

A construção do conjunto de cortes mínimos também não é uma tarefa trivial. Em [FV04] os autores descrevem que o problema de encontrar o conjunto dos cortes mínimos é NP-COMPLETO.

O uso de conjuntos resíduos como forma de depuração de ontologias foi um tópico pouco 
explorado pela comunidade de depuração de ontologias. Por um lado, uma possível explicação para esse fato é a necessidade de construir conjuntos grandes, normalmente maiores do que os conjuntos kernel o que, teoricamente pode acarretar um número maior de chamadas ao mecanismo de inferências.

Por outro lado, muito do que tem sido feito pela comunidade está centrado na ideia do cálculo de justificativas para uma dada inferência, como em [Hor11, HPS08, KPHS07, MMV11]. Nessas abordagens, a construção de conjuntos Resíduos não é de muita ajuda, uma vez que o objetivo é a depuração de granularidade fina, i.e., o usuário deve fazer a escolha de quais elementos devem ser removidos para remover a inferência indesejada.

Nas seções a seguir vamos detalhar algumas abordagens para a construção direta de conjuntos resíduos, iremos listar suas vantagens e desvantagens e terminaremos mostrando um estudo empírico realizado com dados gerados de forma automatizada.

\subsubsection{Construção de um Único Elemento do Conjunto Resíduo}

Ribeiro mostra em [Rib13] que podemos modelar a construção do conjunto resíduo em uma abordagem que ele chamou de encolhe-expande. Essa abordagem funciona de maneira oposta ao algoritmo Black-box clássico. A primeira fase desse algoritmo remove axiomas até que a inferência indesejada não seja mais válida. Na segunda fase, os axiomas removidos são adicionado um a um. Com esse passo, garantimos que somente axiomas que contribuem para a inferência indesejada sejam removidos.

Resina et al., em [RRW14] mostram uma forma de construção direta de um elemento do conjunto resíduo. Durante a fase de encolhimento ele simplesmente remove todos os axiomas da ontologia e segue para a fase de expansão onde cada um deles é readicionado. O algoritmo utiliza uma abordagem similar ao Black-box apresentado por Horridge, em [Hor11] para a construção de um único elemento do conjunto kernel, onde durante a expansão, todos os axiomas da ontologia são adicionados, sobrando para a fase de encolhimento definir o conjunto que seja minimal com relação a inclusão em conjuntos. Chamaremos essas abordagens de implementação das operações de encolhimento e expansão simples de encolhimento/expansão trivial. Chamaremos as abordagens de encolhimento/expansão nas quais todos os elementos são manipulados um a um de expansão/encolhimento clássico.

Chamaremos essa classe de algoritmos do tipo encolhe-expande como Black-box, uma vez que não precisamos realizar modificações internas ao mecanismo de inferências para calcular um único elemento do conjunto resíduo.

O algoritmo Black-box para cálculo de um único elemento do conjunto resíduo pode ser visto na Listagem 2.7.

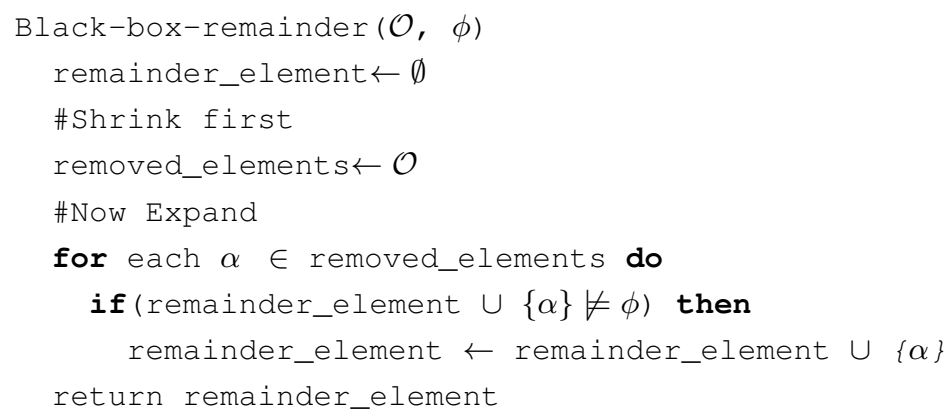


Listagem 2.7: Algoritmo Black-box para Cálculo de um elemento do conjunto resíduo

O algoritmo descrito é provado como correto e completo em [RRW14], porém possui um problema já observado na proposta clássica da abordagem Black-box - possui um número elevado de chamadas ao mecanismo de inferência. Por utilizar a abordagem clássica para realizar a expansão, são necessárias $\mathcal{O}(n)$ chamadas ao mecanismo de inferências.

Para lidar com o problema do alto número de chamadas ao mecanismo de inferência, propomos uma série de otimizações baseadas em heurísticas adaptadas da construção de elementos do conjunto kernel.

Uma variação desse algoritmo pode realizar o encolhimento clássico, como o visto na Listagem 2.7. Essa variação da fase de encolhimento parte da ontologia completa e realiza a remoção gradual de cada um dos seus axiomas, até atingir o ponto onde tal inferência não seja mais válida.

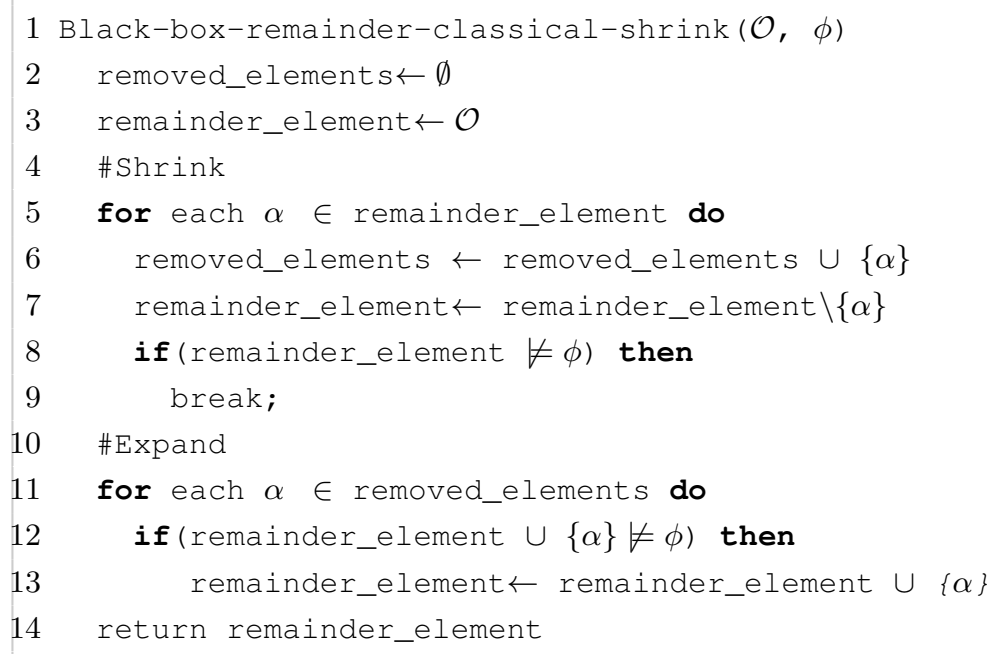

Listagem 2.8: Algoritmo Black-box para Cálculo de um elemento do conjunto resíduo

É fácil de verificar que ambas as abordagens possuem, em uma análise de pior caso, a mesma complexidade. Porém, a construção apresentada em 2.8 pode ser terminada antes que todos os elementos da ontologia sejam testados, como podemos ver no Exemplo 2.9.

Exemplo 2.9 Considere a ontologia:

$$
\begin{aligned}
\mathcal{O}=\{A & \sqsubseteq B, \\
A & \sqsubseteq E, \\
B & \sqsubseteq D, \\
E & \sqsubseteq F\}
\end{aligned}
$$

$A$ partir de $\mathcal{O}$, podemos inferir $A \sqsubseteq D$ e caso essa seja a inferência indesejada, o algoritmo na listagem 2.8 termina na sua primeira iteração, quando, na linha 3, é realizada a remoção do axioma $A \sqsubseteq B$ de $\mathcal{O}$. Após essa remoção a inferência indesejada não é mais válida. Em seguida tentamos realizar uma nova inclusão do que foi removido, nesse caso o axioma $A \sqsubseteq B$, o que resulta no restabelecimento da inferência indesejada. 


\subsubsection{Construção do Conjunto Resíduo Completo}

Apresentamos na seção anterior algoritmos para a construção de elementos únicos do remainder. Nessa seção vamos descrever uma abordagem similar a árvore de cortes mínimos proposta por Reiter [Rei87].

O algoritmo para cálculo do conjunto remainder completo foi proposto por Resina et al. em [RRW14]. A ideia básica é, partindo de um elemento do conjunto remainder, construir novos ramos da árvore, partindo de elementos que não se encontram no nó que dá origem dos ramos. O algoritmo para o cálculo do conjunto remainder completo pode ser visto na Listagem 2.9.

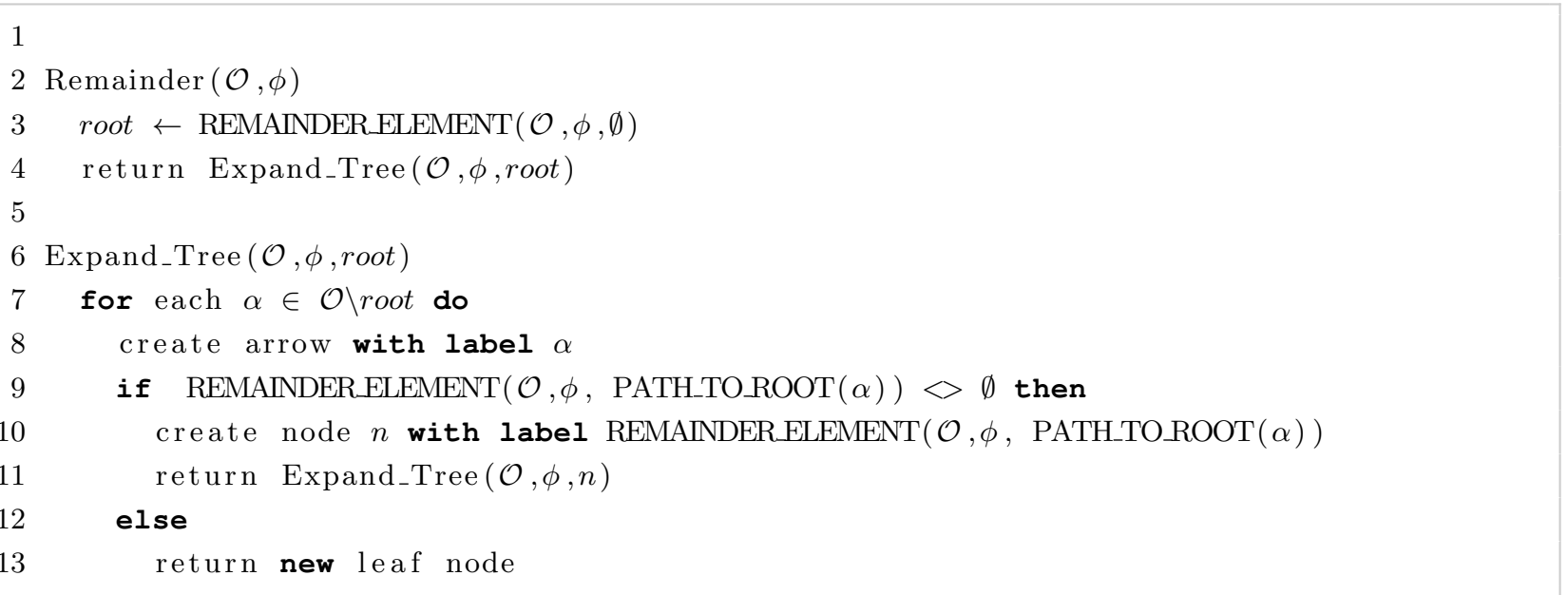

Listagem 2.9: Algoritmo para cálculo do conjunto Remainder

\subsection{Ligação entre a Fusão e a Revisão de Ontologias}

Uma das tarefas fundamentas para a fusão é ter a capacidade de isolar o conjunto de axiomas que são o motivo da inferência indesejada acontecer. A mesma importância é dada a construção de conjuntos resíduos, a partir dos quais podemos encontrar soluções para a inferência indesejada.

Sendo assim, do ponto de vista da fusão de ontologias, a construção tanto de conjunto resíduos como conjuntos kernel são de fundamental importância. Modelamos o nosso processo de acordo com a ideia de que a primeira tarefa a realizar para resolver um conflito é identificar a fonte do conflito. A construção do conjunto kernel serve a esse propósito.

Uma outra forma de lidar com o problema gerado após fusão é utilizar um serviço que tenha a capacidade de lhe sugerir uma possível solução para o problema resultante da fusão. Basicamente, uma solução rápida pode ser construída para o conflito, calculando um único elemento do conjunto resíduo. E, em caso de existir a preferência por axiomas, podemos começar removendo os axiomas menos preferidos. 


\section{Capítulo 3}

\section{Processo para Resolução de Conflitos em Ontologias Pós-Fusão}

O resultado de uma fusão pode não ser o que o usuário está esperando. Sendo esse o caso, normalmente o usuário terá que passar por um processo de depuração de ontologias, localizando informações que podem, mas não deveriam, ser inferidas. O que diferencia esse processo de depuração de um processo de depuração comum é o fato de que após a fusão existem mais chances de ocorrência de inferências indesejadas, ao passo que no processo de definição comum de ontologias, espera-se que o usuário possa realizar checagens de consistências/classificação mais frequentemente, reduzindo o número de conflitos. O Exemplo 3.1 mostra um exemplo de inferência indesejada resultante da união de duas ontologias.

Exemplo 3.1 Suponha que tenhamos as duas ontologias:

$$
\begin{aligned}
\mathcal{O}_{1}=\{\text { Passaro } \sqsubseteq \text { Voa }, \\
\text { Pinguim } \sqsubseteq \text { Passaro }\} \\
\mathcal{O}_{2}=\{\{\text { Tweety }\} \sqsubseteq \text { Pinguim }\}
\end{aligned}
$$

A união dessas duas ontologias resulta em:

$$
\begin{aligned}
& \mathcal{O}=\{\text { Passaro } \sqsubseteq \text { Voa }, \\
& \text { Pinguim } \sqsubseteq \text { Passaro, } \\
&\{\text { Tweety }\} \sqsubseteq \text { Pinguim }\} \models\{\text { Tweety }\} \sqsubseteq V \text { oa }
\end{aligned}
$$

Nesse caso, a inferência $\{$ Tweety $\} \sqsubseteq V o a$ é indesejada pois informa que o indivíduo Tweety, que é um Pinguim, Voa.

Nesse caso, a inferência indesejada é um axioma simples, porém inconsistências $(~ T \sqsubseteq \perp$ ) e incoerências $(C \sqsubseteq \perp)$ também são consideradas inferências indesejadas. Tipicamente, tanto inconsistências/incoerências quanto outras formas de inferências indesejadas são fenômenos provenientes de uma atividade de modelagem mal-realizada. Para nosso processo, no entanto, vamos separar os conflitos originados por esses problemas em: 
1. Conflitos lógicos: ocorrem quando a ontologia em questão se encontra inconsistente ou incoerente. Normalmente esse tipo de conflito é tratado na área de depuração de ontologias inconsistentes/incoerentes [MLB05, Sch05, SHCvH07];

2. Conflitos de modelagem: ocorrem quando, a partir da ontologia em questão, podemos realizar inferências indesejadas. Normalmente esse tipo de conflito é tratado na área de depuração de inferências que não deveriam ser válidas, como o cálculo de justificativas/explicações [MP98, $\left.\mathrm{LBF}^{+} 06\right]$;

Formalmente, conflitos lógicos são formas específicas de conflitos de modelagem, entretanto essa distinção merece ser feita pois ontologias que possuem conflitos lógicos devem ser tratadas de forma especial, uma vez que, segundo o princípio explosivo da lógica clássica, tudo pode ser inferido a partir de uma ontologia inconsistente. Por essa razão, mecanismos de inferência tratam desses casos de forma diferente - nenhuma inferência pode ser calculada a partir dessa ontologia.

Assim, alguns algoritmos descritos na literatura não podem ser aplicados em ontologias inconsistentes. Esse é o caso do algoritmo de ordenação chamado relevância semântica, descrito em [Kal06]. Este algoritmo calcula o número de inferências que são perdidas caso um dado axioma seja retirado da ontologia, medida que não pode ser calculada se o mecanismo de inferência não puder estabelecer o número de inferências não triviais que a ontologia possui antes de realizar a modificação.

Com relação a natureza das inferências indesejadas decorrentes da fusão e do desenvolvimento normal, essas também diferem. Em um processo de construção de ontologias, espera-se que, mesmo que essas inferências ocorram, possuam um escopo menor e mais localizado. Em contrapartida, numa operação de fusão, os focos dessas inferências podem estar distribuídos de forma mais ampla ao longo da ontologia.

Essa distinção serve de motivação para a construção direta de soluções para inferências indesejadas. Por isso focamos, no nosso estudo, em construções que apoiassem o usuário na obtenção dessas soluções.

A identificação de inferências indesejadas pode ser feita usando a técnica de Questões de Competência [NH97, BFS13]. Essa metodologia consiste em definir um conjunto de questões, em linguagem natural, que devem ser respondidas utilizando o conhecimento descrito na ontologia. Essa abordagem é proposta tanto para promover o reúso de ontologias quanto para testar adequação da ontologia a um determinado domínio. No caso do reúso, as questões podem ser utilizadas para selecionar ontologias que se adequem a um domínio específico. No caso do teste de adequação, as questões podem ser utilizadas durante o desenvolvimento de uma ontologia como uma ferramenta de avaliação para o conhecimento que está sendo descrito. Assim, as questões são vistas como requisitos que devem ser atendidos pela ontologia que está sendo reutilizada ou desenvolvida.

Após sofrer fusão, as inferências indesejadas são capturadas na forma de pelo menos uma resposta inadequada a uma questão de competência estabelecida antes do início do processo de fusão. Ou seja, um dos requisitos para a ontologia foi violado.

\subsection{Resolução de Conflitos}

Em [MLB05, MLBP06], os autores lidam com o problema da construção de conjuntos maximalmente consistentes em ontologias. Em $\left[\mathrm{HvHH}^{+} 05\right.$, HS05, Sch05, SHCvH07] os autores estão preo- 
cupados com a chamada depuração de ontologias, onde o objetivo é descobrir a raiz (kernel) de uma inconsistência lógica ou de modelagem (inferências indesejadas). Já em [QP07, Kal06], os autores estão preocupados em lidar com o problema da ordenação de axiomas de acordo com a sua credibilidade ou importância para a ontologia. Por fim, trabalhos como [QLB06, LPSV06, Hor11, Kal06] estão focados no enfraquecimento de axiomas, de forma que não seja necessário descartá-los inteiramente para resolver um conflito lógico ou de modelagem.

Acreditamos que cada um desses aspectos é importante para o resultado da fusão e cada um se aplica a determinadas situações de fusão. Neste trabalho, propomos o agrupamento e encadeamento de algumas das técnicas mais utilizadas na literatura com o objetivo de definirmos um processo interativo para lidar com problemas advindos da fusão. A ideia de agrupar tarefas na forma de processo se assemelha a abordagem utilizada pela ferramenta PROMPT[NM03] para a fusão de ontologias, que também descreve um processo para a realização da fusão.

Sob esse ponto de vista, o trabalho aqui apresentado também se assemelha com o cálculo de planos de reparo, proposto por Kalyanpur [Kal06]. Entretanto, em [Kal06], o autor estava interessado apenas em solucionar incoerências utilizando contrações kernel. Também não era levado em conta o enfraquecimento de axiomas.

O processo aqui proposto entrega ao usuário o poder necessário para construir sua própria metodologia de fusão. Em adição a técnicas descritas na literatura, propomos novas formas para ordenação, construção de conjuntos resíduo, e enfraquecimento de axiomas. O processo proposto pode ser visto na Figura 3.1.

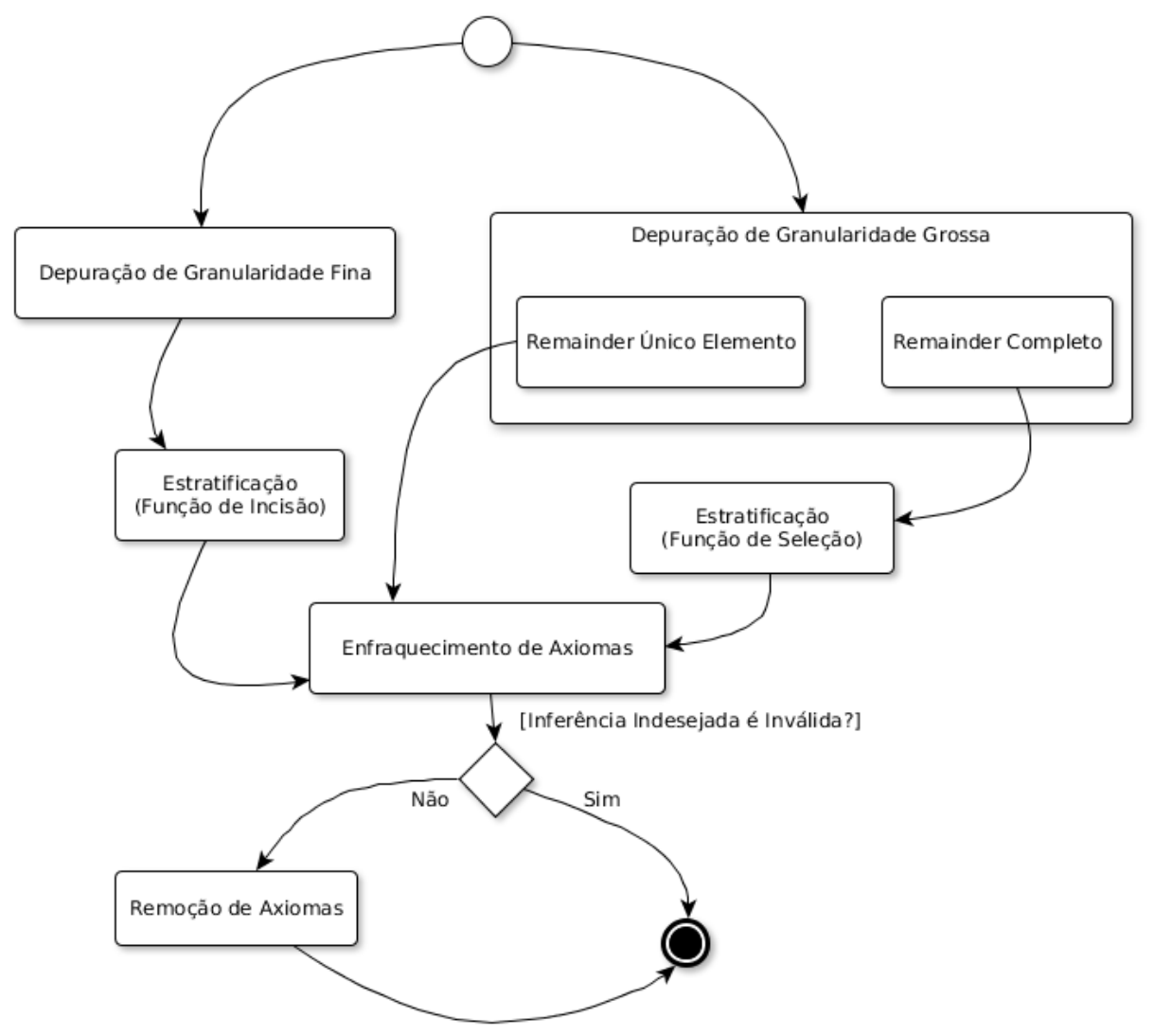

Figura 3.1: Processo de Fusão proposto 
O nosso processo está dividido nas seguintes atividades:

Depuração de Ontologias: o objetivo dessa fase é isolar as causas das inferências indesejadas ou sugerir alternativas que resolvem o problema das inferências, portanto essa fase pode ser realizada de duas formas: com granularidade fina ou grossa, onde a primeira significa que o usuário terá em mãos todas as causas dos conflitos; a segunda pode ainda ser realizada de duas formas: com a construção do conjunto resíduo inteiro ou com a construção de um único elemento do conjunto. Essa fase possui papel fundamental, uma vez que construirá os conjuntos que serão utilizados pelas próximas fases;

Estratificação: tem como objetivo, ordenar os axiomas dentro das ontologias que passarão pela fusão e também ordenar as ontologias seguindo algum critério de importância para o processo de fusão. As atividades desempenhadas nessa fase devem manter o máximo possível de informações que sejam consideradas "relevantes" para o resultado da fusão. A fase de Estratificação é executada somente nos casos de depuração de granularidade fina, onde a estratificação se assemelha ao papel desempenhado por uma função de incisão, vista no Capítulo 2; e de granularidade grossa com o conjunto resíduo inteiro. Nesse caso, a estratificação tem um funcionamento semelhante ao de uma função de seleção, também discutida no Capítulo 2 ;

Enfraquecimento de Axiomas: o enfraquecimento, como visto em [Kal06, Hor11], tem como objetivo manter todos os axiomas envolvidos em conflitos. Esses axiomas sofrem pequenas modificações com o objetivo de restaurar a consistência da ontologia. Chamamos de enfraquecimento, uma vez que essas modificações tem como objetivo reduzir o poder restritivo de um axioma. O enfraquecimento é, muitas vezes, a única forma de modificação necessária para resolução de conflitos como é mostrado em [GH08, QLB06, LPSV06, BKLBW01],

Remoção de Axiomas: algumas vezes, para o usuário, enfraquecer um axioma não faz sentido, por exemplo, quando o conflito é causado por problemas profundos no projeto da ontologia. Pensando nesses casos, adicionamos ao nosso processo a capacidade de remover axiomas responsáveis por inferências indesejada. A ideia de remoção não é nova, ela foi utilizada nas abordagens [MLBP06, MLB05, $\mathrm{HvHH}^{+}$05, Sch05, HV08] como forma de construir ontologias maximalmente consistentes, provendo também algumas pistas a respeito da localização de inconsistências. Essa fase só faz sentido em casos de depuração de granularidade fina, uma vez que, na granularidade grossa, já construímos uma sub-ontologia maximalmente livre da inferência indesejada.

Embora as fases utilizadas entre as formas de depuração sejam as mesmas, as atividades realizadas dentro de cada uma delas são bem diferentes. Portanto, descreveremos 3 instâncias de processos de fusão, um para cada tipo de depuração.

\subsection{Depuração de Granularidade Fina}

A instância de processo para essa forma de depuração pode ser vista na Figura 3.2. A seguir detalharemos cada uma das atividades desenvolvidas em cada fase. 


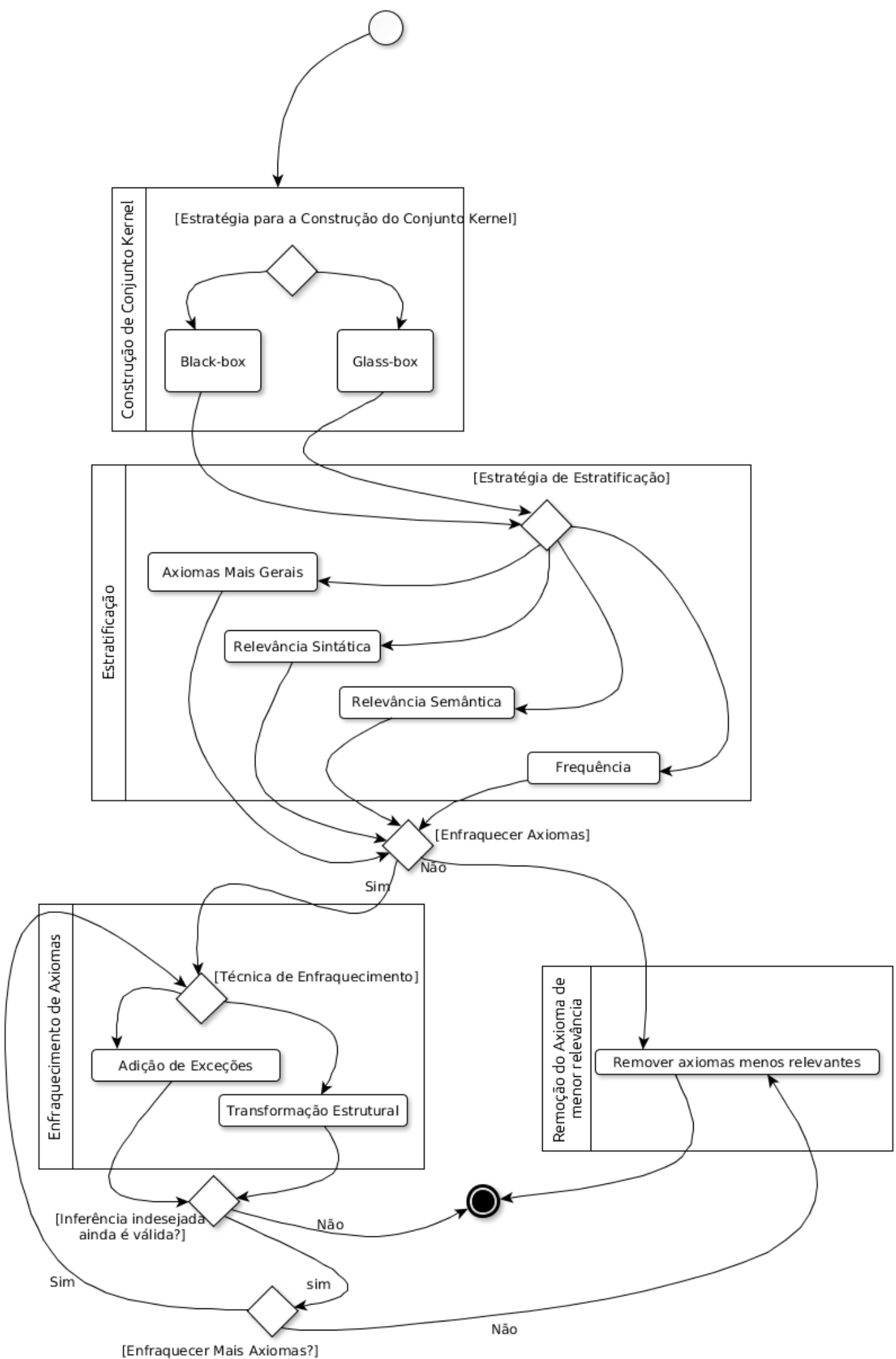

Figura 3.2: Processo de fusão proposto para o caso de depuração de granularidade fina 


\subsubsection{Construção de Conjunto Kernel}

Em [Hor11], Horridge apresenta uma classificação para a tarefa de depuração de granularidade fina sob dois pontos de vista: a capacidade do algoritmo de construir o conjunto kernel inteiro ou um único elemento (chamado de eixo único-todo).

Algoritmos que conseguem construir justificativas únicas para uma consequência lógica são importantes, pois o usuário, ao depurar ontologias, pode realizar tal tarefa de forma iterativa, resolvendo um problema de modelagem por vez. A abordagem é útil quando não possuímos uma função de incisão que removerá de forma automática axiomas envolvidos em conflitos.

Essa forma de depuração não pode ser utilizada para solução de conflitos pós fusão, uma vez que não sabemos previamente a extensão do problema com o qual estamos lidando, assim, um conflito pode possuir várias causas e, ao lidarmos com uma única, não necessariamente estamos eliminando o conflito. Por esse motivo, no nosso processo realizamos a construção de conjuntos kernel completos durante a etapa de depuração. Embora em muitos casos, seja melhor ter uma visão geral do conflito, construindo o conjunto kernel inteiro, uma alternativa a essa construção seria executar o processo de forma iterativa, onde cada ciclo seria utilizado para a resolução de uma das fontes de conflito.

O cálculo do conjunto kernel por si só não implica na resolução do(s) conflito(s). Para que a resolução possa ser executada de forma automática, o processo ainda ainda precisa de uma função de incisão, que escolherá axiomas dentro dos $\alpha$-kernels. Essas funções são construídas de acordo com a estratégia de priorização escolhida pelo usuário e podem, potencialmente, aumentar a complexidade da estratégia de priorização.

O segundo ponto de vista nessa classificação mede o nível de dependência do mecanismo de inferências utilizado, sendo um dos extremos o algoritmo Glass-box, completamente dependente do mecanismo de inferência; e no outro extremo o Black-box [PSK05], completamente independente de mecanismos de inferência. Essas abordagens foram discutidas no Capitulo 2.

O extremo Glass-box dessa classificação contém algoritmos que são modificações de mecanismos de inferência. Essas modificações podem, por exemplo, manter armazenados conjuntos (ramos no tableaux) como em [MLB05] utilizados para a geração de uma contradição. Embora seja uma abordagem de melhor desempenho, é necessário realizar modificações no mecanismo de inferência, tarefa que não é trivial, dado que os mecanismos de inferência estão em constante evolução.

No extremo Black-box se encontram algoritmos que são independentes de mecanismos de inferência. Tipicamente são algoritmos mais custosos que algoritmos Glass-box que calculam os conjuntos conflitos em uma única chamada. Em algoritmos Black-box são necessárias várias chamadas ao mecanismo de inferências para construir um único conjunto conflito, o que pode ser ineficiente em termos de tempo de execução, mas simples de implementar uma vez que o algoritmo somente faz consultas ao mecanismo de inferência.

No nosso processo deixamos espaço para o usuário escolher entre uma abordagem Black-box e uma Glass-box. A escolha deve levar em consideração alguns fatores:

- Lógica utilizada para representar a ontologia: dependendo da lógica utilizada para representar a ontologia, o algoritmo utilizado pelo mecanismo de inferência pode ser mais ou menos sofisticado, dificultando as mudanças ao mesmo.

- Tamanho da ontologia pós-fusão: a tarefa de classificação ou checagem de consistência re- 
alizada pelo mecanismo de inferência pode variar também de acordo com o tamanho da ontologia pós-fusão. Normalmente esses dados são mantidos diretamente na memória, então a operação de carga e descarga da ontologia na memória, realizada no algoritmo Black-box pode resultar em perda de desempenho;

No fim dessa atividade devemos ter construído o conjunto kernel.

\subsubsection{Estratificação}

A próxima atividade a ser desempenhada durante o processo de fusão é a estratificação. A adoção dessa tarefa foi inspirada pelos trabalhos [QLB06, QP07, MLB05, MLBP06]. Esses trabalhos possuem em comum uma característica: as ontologias a passarem pela fusão estão ordenadas por preferência ou confiabilidade. Esse processo é chamado de estratificação. Benferhat et al. [BCD $\left.{ }^{+} 93\right]$ definem uma base de conhecimento priorizada (ou estratificada) $K$ como possuindo uma pré-ordem total, ou seja, uma relação transitiva e reflexiva $\leq$, onde para cada dois elementos $x$ e $y$ em $K$, ou $x \leq y$ ou $y \leq x$. Essa relação representa a preferência de alguns axiomas em detrimento de outros. Dessa forma, uma base de conhecimento $K$ pode ser vista como sendo $K=\left\{S_{1}, S_{2}, \ldots, S_{n}\right\}$, onde $S_{i}$ representa o conjunto de axiomas i-preferidos.

\subsubsection{Priorização de Axiomas Mais Específicos}

No processo proposto, disponibilizamos algumas técnicas para a estratificação automatizada das bases de conhecimento, a primeira delas se chama Priorização de Axiomas mais Específicos. Essa técnica foi proposta em [QP07] e consiste basicamente em colocar maior peso nos axiomas que são considerados como descrevendo conceitos mais específicos que outros. Em [QP07], os autores definem essa relação de especificidade como: um axioma $\alpha_{1}$ da forma $C_{1} \sqsubseteq D_{1}$ é mais específico (menos geral) que um axioma $\alpha_{2}$ da forma $C_{2} \sqsubseteq D_{2}$ se e somente se $C_{1} \sqsubseteq C_{2}$ e $C_{2} \nsubseteq C_{1}$. A ideia de priorização de axiomas mais específicos foi primeiro proposta por Benferhat [BEBC03] como uma forma de resolver conflitos em políticas de segurança para a restrição de acesso a sistemas.

No entanto, a abordagem descrita por Qi et al. em [QP07] se restringe somente ao conhecimento terminológico contido em uma ontologia, o que significa que a abordagem não trata dos indivíduos, que são considerados, segundo os autores, como axiomas seguros, i.e., que estão corretos. Tal comportamento pode ser simulado, acrescentando os indivíduos a um estrato de mais baixa ordem. Em nosso processo, adicionamos o algoritmo proposto por [QP07], assim como uma versão modificada que prioriza axiomas mais gerais em vez de mais específicos. Ao utilizar essa versão modificada do algoritmo o usuário poderá tentar manter os axiomas de mais alto nível, que possivelmente impõem mais restrições e acrescentam mais propriedades ao domínio inteiro do que os axiomas mais específicos. O Exemplo 3.2 ilustra o funcionamento da abordagem proposta em [QP07].

Exemplo 3.2 Consideremos a ontologia

$$
\begin{aligned}
\mathcal{O}=\left\{C_{2}\right. & \sqsubseteq C_{1}, \\
& C_{3} \sqsubseteq C_{1} \\
& C_{1} \sqsubseteq \neg C_{4}, \\
& \left.C_{2} \sqsubseteq C_{4}\right\} \models C_{2} \sqsubseteq \perp
\end{aligned}
$$


Calculando o conjunto kernel $\mathcal{K}$ considerando $\alpha=C_{2} \sqsubseteq \perp$ obteríamos:

$$
\mathcal{O} \Perp\left(C_{2} \sqsubseteq \perp\right)=\left\{C_{1} \sqsubseteq \neg C_{4}, C_{2} \sqsubseteq C_{1}, C 2 \sqsubseteq C_{4}\right\}
$$

Uma função de estratificação $\sigma_{\text {general }}$ baseada na estratégia de estratificação proposta por [QP07] aplicada ao kernel $\mathcal{K}$ resultaria em:

$$
\begin{aligned}
\sigma_{\text {general }}(\mathcal{K})=\{ & \left\{C_{2} \sqsubseteq C_{1}, C_{2} \sqsubseteq C_{4}\right\}, \\
& \left.\left\{C_{1} \sqsubseteq \neg C_{4}\right\}\right\}
\end{aligned}
$$

Uma função de incisão que removesse do conjunto kernel axiomas em estratos mais baixos, removeria o axioma $C_{1} \sqsubseteq \neg C_{4}$, resultando na ontologia i.e.,

$$
\mathcal{R}=\left\{C_{2} \sqsubseteq C_{1}, C_{3} \sqsubseteq C_{1}, C_{2} \sqsubseteq C_{4}\right\}
$$

O exemplo 3.2 demonstra como a remoção de axiomas mais gerais pode remover as restrições impostas a mais conceitos presentes no domínio e não envolvidos na inconsistência. No Exemplo 3.2 , a remoção do axioma $C_{1} \sqsubseteq \neg C_{4}$ teria impacto também sobre o axioma $C_{3} \sqsubseteq C_{1}$, uma vez que este impõe a disjunção do conceito $C_{4}$ a todos os descendentes de $C_{1}$. O conceito $C_{3}$ poderia ter um grande conjunto de subconceitos, para os quais a restrição de disjunção pode ser importante. $\mathrm{O}$ Exemplo 3.3 (continuação do Exemplo 3.2) demonstra o funcionamento do algoritmo modificado.

Exemplo 3.3 Ao utilizarmos uma função de estratificação inversa a proposta por [QP07], o resultado da estratificação seria o kernel estratificado:

$$
\begin{aligned}
\sigma_{\text {general }^{-}}\left(\mathcal{K}^{\prime}\right)=\{ & \left\{C_{1} \sqsubseteq \neg C_{4}\right\}, \\
& \left.\left\{C_{2} \sqsubseteq C_{1}, C_{2} \sqsubseteq C_{4}\right\}\right\}
\end{aligned}
$$

Um algoritmo que utilizasse uma função de incisão que removesse do conjunto kernel axiomas em estratos de mais baixa ordem, daria duas opções de solução ao usuário:

$$
\mathcal{R}_{1}=\left\{C_{1} \sqsubseteq \neg C_{4}, C_{2} \sqsubseteq C_{1}, C_{3} \sqsubseteq C_{1}\right\}
$$

ou

$$
\mathcal{R}_{2}=\left\{C_{1} \sqsubseteq \neg C_{4}, C_{3} \sqsubseteq C_{1}, C_{2} \sqsubseteq C_{4}\right\}
$$

A escolha pode ser feita pelo usuário manualmente ou ele/ela pode fazer uso de uma outra forma de estratificação para escolher a ontologia que possua o maior número de axiomas em estratos de mais alto nível.

É possível ver que a escolha por priorizar axiomas mais gerais possibilitou mantermos uma restrição possivelmente importante sobre o domínio inteiro descrito. O motivo pelo qual mantemos as duas abordagens é o simples fato de que para alguns sistemas, conceitos mais específicos são mais 
importantes, e.g., sistemas de busca. Nesses sistemas, em geral, termos menos genéricos resultam em resultados mais precisos. Um outro contexto no qual a priorização de axiomas mais específicos também se encaixaria é o da definição de política de segurança para acesso a sistemas, como proposto em [BEBC03].

\subsubsection{Ordenar por Frequência}

Ordenar por frequência: contar o número de conjuntos conflito nos quais o axioma aparece. Se o axioma aparece em $n$ conjuntos, caso seja removido será possível solucionar $n$ conflitos. Essa técnica pode ser utilizada tanto por ontologias incoerentes quanto inconsistentes. $\mathrm{O}$ funcionamento dessa estratégia é ilustrado no Exemplo 3.4.

Exemplo 3.4 Suponha a ontologia:

$$
\begin{aligned}
\mathcal{O}=\{A & \sqsubseteq B, \\
B & \sqsubseteq C, \\
B & \sqsubseteq D, \\
D & \sqsubseteq C\} \mid A \sqsubseteq C
\end{aligned}
$$

Construindo o conjunto kernel $\mathcal{K}$ para $\mathcal{O}$, supondo $\alpha=A \sqsubseteq C$ obtemos:

$$
\begin{aligned}
\mathcal{O} \Perp(A \sqsubseteq C)=\{ & \{A \sqsubseteq B, B \sqsubseteq C\}, \\
& \{A \sqsubseteq B, B \sqsubseteq D, D \sqsubseteq C\}\}
\end{aligned}
$$

A função de estratificação $\sigma_{\text {freq }}$ baseada na ideia de frequência apresentado por Kalyanpur, em [Kal06] construiria o kernel estratificado:

$$
\begin{aligned}
\sigma_{\text {freq }}(\mathcal{K})=\{ & \{\{A \sqsubseteq B\},\{B \sqsubseteq C\}\}, \\
& \{\{A \sqsubseteq B\},\{B \sqsubseteq D, D \sqsubseteq C\}\}\}
\end{aligned}
$$

Uma função de incisão que utilizasse essa estratégia de estratificação, escolheria o elemento que mais se repete em todos os $\mathcal{K}_{i}$ elementos do conjunto kernel $\mathcal{K}$. Dessa forma, o axioma escolhido para a remoção seria $A \sqsubseteq B$, que resolveria ambas as causas da inferência indesejada, removendo um único axioma.

Ao utilizarmos essa estratégia de estratificação, garantimos que a remoção da informação seja a quantidade mínima necessária para remover a inferência indesejada. No caso, a minimalidade aqui se refere ao número de axiomas.

\subsubsection{Ordenar por Relevância Semântica}

Ordenar por relevância semântica: o número de inferências que são perdidas se um axioma é removido, i.e., o número de relacionamentos de sub-classes que são adicionados ou removidos caso o axioma seja removido da ontologia. Quanto maior o número de inferências menor prioridade tem o axioma na remoção. Essa técnica não pode ser utilizada em ontologias inconsistentes, pois se faz 
necessário utilizar o reasoner e calcular inferências prévias e posteriores i.e., a inferência indesejada não pode ser do tipo $\alpha=\top \sqsubseteq \perp$. O Exemplo 3.5 ilustra o funcionamento de uma função de incisão que faz uso dessa estratégia de estratificação.

Exemplo 3.5 Considerando a ontologia:

$$
\begin{aligned}
\mathcal{O}=\{A & \sqsubseteq B, \\
B & \sqsubseteq C, \\
C & \sqsubseteq D, \\
A & \sqsubseteq E, \\
E & \sqsubseteq D\} \models A \sqsubseteq D
\end{aligned}
$$

Ao construirmos o conjunto kernel em $\mathcal{O}$, considerando a inferência indesejada $\alpha=A \sqsubseteq D$, obtemos:

$$
\begin{gathered}
\mathcal{O} \Perp(A \sqsubseteq D)=\{\{A \sqsubseteq B, B \sqsubseteq C, C \sqsubseteq D\}, \\
\{A \sqsubseteq E, E \sqsubseteq D\}\}
\end{gathered}
$$

Vamos utilizar a função $\sigma_{\text {semantic }}$, baseada na medida de relevância semântica, para realizarmos a estratificação e o resultado é:

$$
\begin{aligned}
\sigma_{\text {semantic }}(\mathcal{K})=\{\{\{B \sqsubseteq C\},\{A \sqsubseteq B\},\{C \sqsubseteq D\}\}, \\
\\
\{\{A \sqsubseteq E, E \sqsubseteq D\}\}\}
\end{aligned}
$$

O primeiro elemento do conjunto kernel $\mathcal{K}, \mathcal{K}_{1}$ possui três estratos, $\mathcal{S}_{1}=\{B \sqsubseteq C\}, \mathcal{S}_{2}=\{A \sqsubseteq$ $B\}$ e $\mathcal{S}_{3}=\{C \sqsubseteq D\}$. Em $\mathcal{S}_{1}$, o número de inferências invalidadas caso algum dos axiomas seja removido é três, pois as inferências $A \sqsubseteq C, A \sqsubseteq D$ e $B \sqsubseteq D$ são quebradas. Em $\mathcal{S}_{2}$, somente $A \sqsubseteq C$ a $A \sqsubseteq D$ são quebradas. No terceiro estrato, somente uma inferência é quebrada, i.e., $A \sqsubseteq D$. Considerando que uma das inferências quebradas é a inferência indesejada, o número de inferências quebradas é reduzido em um em todos os estratos.

Em $\mathcal{K}_{2}$, temos um único estrato, $\mathcal{S}_{1}$, que caso algum dos seus elementos seja removido, uma única inferência será quebrada, i.e., $A \sqsubseteq D$. Nesse caso, a mesma consideração com relação a invalidação das inferências deve ser feita, i.e. a única inferência quebrada é a inferência indesejada, sendo assim, o número de inferências invalidadas é reduzido em um.

Uma função de incisão que utilizasse a função $\sigma_{\text {semantic }}$ de estratificação escolheria o único elemento do estrato $\mathcal{S}_{3}$ em $\mathcal{K}_{1}$ e escolheria um elemento em $\mathcal{S}_{1}$ em $\mathcal{K}_{2}$. Essa escolha pode inclusive ser guiada por uma segunda estratégia de estratificação. Com isso a única inferência perdida foi a inferência indesejada.

\subsubsection{Ordenar por Relevância Sintática}

Essa estratégia permite que o usuário escolha preservar axiomas que compartilhem conceitos com o maior número de outros axiomas, uma vez que isso pode indicar que tais conceitos são importantes na descrição de um dado domínio. Para utilizar essa técnica deve-se analisar também 
o número de indivíduos que em suas descrições também compartilham conceitos com o axioma que está sendo ordenado.

A preocupação com essa atividade é remover o trecho de informação que está menos conectada (estruturalmente) com o resto da ontologia. O funcionamento dessa estratégia de estratificação é visto no Exemplo 3.6.

Exemplo 3.6 Suponha a ontologia:

$$
\begin{aligned}
\mathcal{O}=\{A & \sqsubseteq B, \\
B & \sqsubseteq C, \\
B & \sqsubseteq E, \\
C & \sqsubseteq D, \\
& E \sqsubseteq D\} \models A \sqsubseteq D
\end{aligned}
$$

Considerando o axioma indesejado $\alpha=A \sqsubseteq D$, calculando o conjunto kernel $\mathcal{K}$ obtemos:

$$
\begin{aligned}
\mathcal{O} \Perp(A \sqsubseteq D)=\{ & \{A \sqsubseteq B, B \sqsubseteq C, C \sqsubseteq D\}, \\
& \{A \sqsubseteq B, B \sqsubseteq E, E \sqsubseteq D\}\}
\end{aligned}
$$

Ao utilizar uma função $\sigma_{\text {syntactic }}$ que realiza a estratificação de $\mathcal{K}$, obtemos:

$$
\begin{aligned}
\sigma_{\text {syntactic }}(\mathcal{K})=\{ & \{\{B \sqsubseteq C\},\{A \sqsubseteq B, C \sqsubseteq D\}\}, \\
& \{\{B \sqsubseteq E\},\{A \sqsubseteq B, E \sqsubseteq D\}\}\}
\end{aligned}
$$

$O$ primeiro $\alpha$-kernel, $\mathcal{K}_{1}$ possui dois estratos, $\mathcal{S}_{1}$, com um único elemento $B \sqsubseteq C$, que está sintaticamente conectado $a A \sqsubseteq B, B \sqsubseteq E$, $e C \sqsubseteq D$; e $\mathcal{S}_{2}$, que possui dois elementos: $A \sqsubseteq B$ que está conectado a $B \sqsubseteq C$ e $B \sqsubseteq E$; e $C \sqsubseteq D$ que está conectado a $B \sqsubseteq C$ e $E \sqsubseteq D$.

De forma análoga, o segundo $\alpha$-kernel, $\mathcal{K}_{2}$ possui dois estratos, $\mathcal{S}_{1}$, com um único elemento $B \sqsubseteq E$, que está sintaticamente conectado a $A \sqsubseteq B, B \sqsubseteq E$, e $E \sqsubseteq D$; e $\mathcal{S}_{2}$, que possui dois elementos: $A \sqsubseteq B$ que está conectado a $B \sqsubseteq C$ e $B \sqsubseteq E$; e $E \sqsubseteq D$ que está conectado $a B \sqsubseteq E$ e $C \sqsubseteq D$.

Uma função de incisão que utilizasse a função $\sigma_{\text {syntactic }}$ de estratificação escolheria um elemento em $\mathcal{S}_{2}$ em $\mathcal{K}_{1}$ e $\mathcal{K}_{2}$. O critério de escolha poderia ser a combinação com outra função de estratificação, e.g., utilizando o critério da frequência, o axioma escolhido seria $A \sqsubseteq B$.

O fim dessa fase produz uma ontologia com seus axiomas distribuídos em estratos e classificados de acordo com o nível de preferência.

\subsection{Depuração de Granularidade Grossa Completa}

O processo para fusão de ontologias que parte da depuração com granularidade grossa, levando em conta o conjunto resíduo inteiro pode ser visto na Figura 3.3 


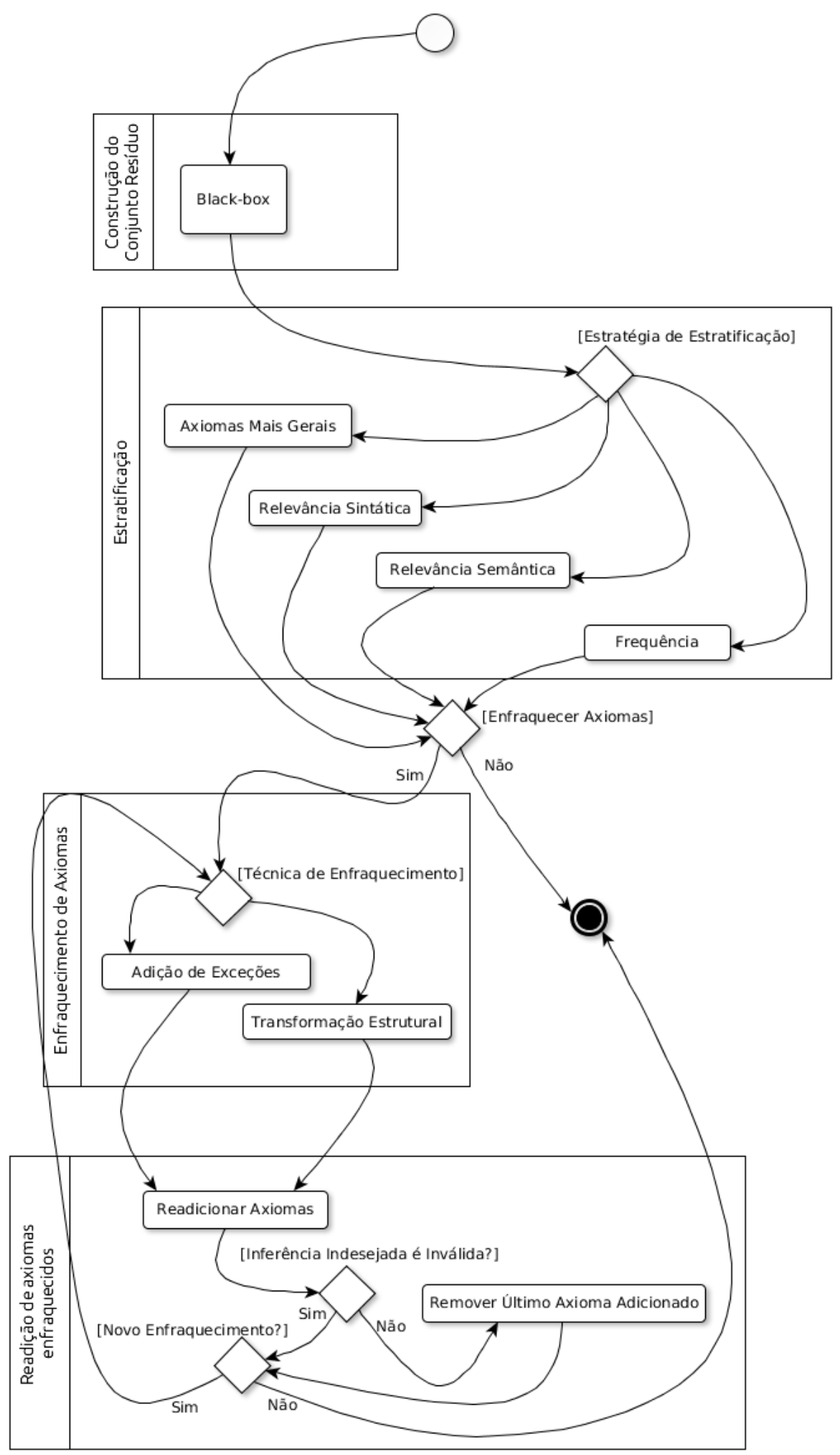

Figura 3.3: Processo de fusão proposto para o caso de depuração de granularidade grossa com o conjunto resíduo completo 


\subsubsection{Construção de Conjunto Resíduo}

A principal atividade desenvolvida durante a depuração de granularidade grossa completa é a construção do conjunto resíduo. Como visto no Capítulo 2, o conjunto resíduo contém todas as subontologias maximais livres de conflitos. Esses conjuntos são utilizados como reparos, individuais, para cada conflito presente na ontologia.

No nosso processo caracterizamos a construção desses conjuntos - fazendo um paralelo a estratégia de expande-encolhe para a construção do conjunto kernel - utilizando uma estratégia encolhe-expande. No Capítulo 4 apresentamos uma série de construções baseadas em heurísticas que buscam otimizar a construção do conjunto resíduo. Essas heurísticas foram colocadas a prova contra dados reais e gerados automaticamente.

O melhor resultado alcançado, considerando dados reais foi a utilização da estratégia de encolhimento baseada na noção de conectividade sintática e a estratégia de expansão clássica, onde os elementos são readicionados um-a-um. Portanto, essa é a estratégia recomendada no processo para a construção de um único elemento do conjunto Resíduo.

A construção do conjunto resíduo inteiro também pode ser otimizada, seguindo os mesmos passos das otimizações descritas por Horridge [Hor11] ao algoritmo de Reiter. Ambas estratégias fechamento precoce de caminhos e reaproveitamento de nós - também se aplicam na construção do conjunto resíduo inteiro. Assim, a recomendação, no nosso processo, é de utilizar ambas otimizações para a construção do conjunto resíduo inteiro.

Uma outra forma de construir o conjunto resíduo é a partir do conjunto kernel obtido utilizando o algoritmo de Reiter. Na árvore de cortes mínimos, o conjunto resíduo para uma dada inferência é calculado recuperando os rótulos das arestas que ligam um nó folha a raiz e em seguida subtraindo esse conjunto de axiomas da ontologia completa. Se realizarmos esse processo para todos os nós folhas obteremos o conjunto resíduo inteiro.

Sendo assim, mesmo nos casos em que construir um conjunto kernel é menos custoso computacionalmente, conseguimos construir um conjunto resíduo sem aumentos na ordem de complexidade.

\subsubsection{Estratificação}

Após a construção do conjunto resíduo completo, é hora de selecionarmos o elemento que mais se aproxima com o que é desejado pelo usuário. Esse papel é exercido por uma função de seleção [AGM85]. Aqui nesta seção descrevemos como utilizamos os conceitos descritos nas funções de incisão descritas por Kalyanpur em [Kal06] e Qi et al. em [QP07] e construímos funções de seleção a partir delas.

No nosso processo, antes da seleção de um dos elementos do conjunto resíduo, os mesmos são ordenados utilizando alguma estratégia de estratificação [QP07]. Essa operação divide um conjunto de axiomas em estratos, que são por sua vez subconjuntos do conjunto original. Esses elementos são ordenados por ordem de preferência. Assim, a função de seleção escolherá o item do conjunto resíduo que contiver o estrato de nível mais elevado e secundariamente o que possuir o maior número de axiomas nesse nível. Daremos o nome de conjunto resíduo estratificado àquele que passou pelo processo de estratificação. 


\subsubsection{Seleção Baseada na Generalidade dos Axiomas}

Em [QP07], Qi et al. propõem o uso da noção de generalidade de um axioma como uma função de ordenação sobre os mesmos. Na Seção 3.2.2.1 descrevemos como funciona a estratificação (incisão) baseada nessa noção de generalidade. Vimos que pode ser o caso que a relação se inverta e precisemos preferir axiomas mais específicos em detrimento dos mais gerais. Para a estratificação (seleção) em uma depuração de granularidade grossa o mesmo princípio se aplica.

A aplicação do conceito se dá de forma direta, podemos estratificar todos os elementos do conjunto resíduo, em seguida selecionar aquele que tiver o maior número de estratos, que indica que possui mais níveis na hierarquia da especificidade de conceitos. O critério de desempate, caso o número de estratos seja o mesmo para mais que um elemento no conjunto resíduo é selecionar aquele que possui o maior número de elementos no estrato de mais alto nível.

O exemplo 3.7 ilustra o funcionamento da ideia.

Exemplo 3.7 Suponha a seguinte ontologia $\mathcal{O}$ :

$$
\begin{aligned}
\mathcal{O}=\{A & \sqsubseteq B \\
B & \sqsubseteq C \\
C & \sqsubseteq E \\
A & \sqsubseteq F \\
& F \sqsubseteq E\} \models A \sqsubseteq E
\end{aligned}
$$

Considerando a inferência indesejada $\alpha=A \sqsubseteq E$, o conjunto resíduo $\mathcal{R}=\mathcal{O} \perp \alpha$ obtemos:

$$
\begin{aligned}
\mathcal{O} \perp A \sqsubseteq E=\{ & \{A \sqsubseteq B, B \sqsubseteq C, A \sqsubseteq F\}, \\
& \{A \sqsubseteq B, B \sqsubseteq C, F \sqsubseteq E\}, \\
& \{B \sqsubseteq C, C \sqsubseteq E, A \sqsubseteq F\}, \\
& \{B \sqsubseteq C, C \sqsubseteq E, F \sqsubseteq E\}, \\
& \{A \sqsubseteq B, C \sqsubseteq E, A \sqsubseteq F\}, \\
& \{A \sqsubseteq B, C \sqsubseteq E, F \sqsubseteq E\}\}
\end{aligned}
$$

O resultado da estratificação no conjunto resíduo, utilizando a função $\gamma_{\text {general }}$ seria:

$$
\begin{aligned}
\gamma_{\text {general }}(\mathcal{R})=\{ & \{\{A \sqsubseteq B, A \sqsubseteq F\},\{B \sqsubseteq C\}\}, \\
& \{\{A \sqsubseteq B\},\{B \sqsubseteq C, F \sqsubseteq E\}\}, \\
& \{\{B \sqsubseteq C\},\{C \sqsubseteq E, A \sqsubseteq F\}\}, \\
& \{\{B \sqsubseteq C\},\{C \sqsubseteq E, F \sqsubseteq E\}\}, \\
& \{\{A \sqsubseteq B, C \sqsubseteq E, A \sqsubseteq F\}\}, \\
& \{\{A \sqsubseteq B, C \sqsubseteq E, F \sqsubseteq E\}\}\}
\end{aligned}
$$

A função de estratificação $\gamma_{\text {general }}$ funciona contando o número de axiomas que descrevem conceito de mesmo grau de especificidade, e.g., para $\mathcal{R}_{1}=\{A \sqsubseteq B, B \sqsubseteq C, A \sqsubseteq F\}$ temos que os axiomas $A \sqsubseteq B$ e $A \sqsubseteq F$, são mais específicos que $B \sqsubseteq C$, uma vez que $A \sqsubseteq B$. Para 
$\mathcal{R}_{2}=\{A \sqsubseteq B, B \sqsubseteq C, F \sqsubseteq E\}$ temos que o axioma mais específico é $A \sqsubseteq B$, uma vez que em $B \sqsubseteq C$ temos $A \sqsubseteq B$ e $F \sqsubseteq E$ que não se relaciona .

Assim, após a estratificação, estabelecemos a ordem de preferência no conjunto resíduo em questão, onde o elemento de maior precedência (o mais preferivel) é $\mathcal{R}_{1}=\{A \sqsubseteq B, B \sqsubseteq C, A \sqsubseteq F\}$, com 2 estratos: $\mathcal{S}_{1}=\{A \sqsubseteq B, A \sqsubseteq F\}$ e $\mathcal{S}_{2}=\{B \sqsubseteq C\}$. Existem outros itens do conjunto resíduo que possuem o mesmo número de estratos, porém $\mathcal{R}_{1}$ possui o maior número de elementos no estrato de mais alta ordem.

Assim como no caso da depuração de granularidade fina, pode ser o caso que a seleção de elementos deva dar preferência a axiomas mais específicos. Sendo esse o caso, a execução reversa do algoritmo pode ser utilizada, i.e., selecionamos o elemento do conjunto resíduo que possui o maior número de elementos no estrato de mais baixa precedência. Nesse caso também é necessário realizar a seleção dos itens do conjunto resíduo que possuem o maior número de estratos. O exemplo 3.8 mostra o uso dessa estratégia.

Exemplo 3.8 (continuação do exemplo 3.7) Para o conjunto resíduo:

$$
\begin{aligned}
\mathcal{O} \perp A \sqsubseteq E=\{ & \{A \sqsubseteq B, B \sqsubseteq C, A \sqsubseteq F\}, \\
& \{A \sqsubseteq B, B \sqsubseteq C, F \sqsubseteq E\}, \\
& \{B \sqsubseteq C, C \sqsubseteq E, A \sqsubseteq F\}, \\
& \{B \sqsubseteq C, C \sqsubseteq E, F \sqsubseteq E\}, \\
& \{A \sqsubseteq B, C \sqsubseteq E, A \sqsubseteq F\}, \\
& \{A \sqsubseteq B, C \sqsubseteq E, F \sqsubseteq E\}\}
\end{aligned}
$$

Supondo um novo operador de estratificação $\gamma_{\text {rev }}$ que executa o mesmo algoritmo de $\gamma$ sendo que em ordem reversa. Ao aplicarmos $\gamma_{\text {rev }}$ ao conjunto resíduo $\mathcal{R}=\mathcal{O} \perp A \sqsubseteq E$ obtemos:

$$
\begin{aligned}
\gamma_{r e v}(\mathcal{R})=\{ & \{\{B \sqsubseteq C\},\{A \sqsubseteq B, A \sqsubseteq F\}\}, \\
& \{\{B \sqsubseteq C, F \sqsubseteq E\},\{A \sqsubseteq B\}\}, \\
& \{\{C \sqsubseteq E, A \sqsubseteq F\},\{B \sqsubseteq C\}\}, \\
& \{\{C \sqsubseteq E, F \sqsubseteq E\},\{B \sqsubseteq C\}\}, \\
& \{\{A \sqsubseteq B, C \sqsubseteq E, A \sqsubseteq F\}\}, \\
& \{\{A \sqsubseteq B, C \sqsubseteq E, F \sqsubseteq E\}\}\}
\end{aligned}
$$

Ao realizarmos a seleção pelos itens que possuem a maior quantidade de estratos obtemos: $\mathcal{S}_{1}=\{\{B \sqsubseteq C\},\{A \sqsubseteq B, A \sqsubseteq F\}\}, \mathcal{S}_{2}=\{\{B \sqsubseteq C, F \sqsubseteq E\},\{A \sqsubseteq B\}\}, \mathcal{S}_{3}=\{\{C \sqsubseteq E, A \sqsubseteq$ $F\},\{B \sqsubseteq C\}\}$ e $\mathcal{S}_{4}=\{\{C \sqsubseteq E, F \sqsubseteq E\},\{B \sqsubseteq C\}\}$. Após o primeiro passo de seleção, filtramos os conjuntos que possuem o maior número de itens e obtemos $\mathcal{S}_{2}, \mathcal{S}_{3}, \mathcal{S}_{4}$. Desse ponto em diante, o usuário pode escolher uma segunda forma de estratificação ou selecionar, manualmente uma das opções uma vez que as 3 alternativas são igualmente aceitáveis. Alternativamente o usuário pode escolher ficar com as 3 formas e então utilizamos como resposta a intersecção das 3. 


\subsubsection{Seleção Baseada em Frequência}

O objetivo dessa atividade de estratificação é priorizar elementos do conjunto resíduo cujos axiomas ocorrem no maior número de elementos do conjunto. Dessa forma, acreditamos que estamos priorizando a escolha que melhor representa as possíveis soluções para o conflito. Utilizando essa abordagem nos aproximamos do funcionamento de operadores de majoração descritos em [LS98, LS95].

A abordagem baseada em majoração tem como objetivo minimizar a insatisfação global, o que é atingido quando selecionamos o item que possui o maior número de elementos compartilhados.

Exemplo 3.9 Suponha a seguinte ontologia

$$
\begin{aligned}
\mathcal{O}=\{A & \sqsubseteq B, \\
B & \sqsubseteq C, \\
C & \sqsubseteq D, \\
B & \sqsubseteq E, \\
& E \sqsubseteq D\} \models A \sqsubseteq D
\end{aligned}
$$

Considerando a inferência indesejada $\alpha=A \sqsubseteq D$, ao calcularmos o conjunto resíduo $\mathcal{R}=\mathcal{O} \perp(A \sqsubseteq$ D) obtemos:

$$
\begin{aligned}
& \mathcal{O} \perp(A \sqsubseteq D)=\{\{A \sqsubseteq B, B \sqsubseteq C, B \sqsubseteq E\}, \\
& \{A \sqsubseteq B, B \sqsubseteq C, E \sqsubseteq D\}, \\
& \{A \sqsubseteq B, C \sqsubseteq D, B \sqsubseteq E\}, \\
& \{A \sqsubseteq B, C \sqsubseteq D, E \sqsubseteq D\}, \\
& \{B \sqsubseteq C, C \sqsubseteq D, B \sqsubseteq E, E \sqsubseteq D\}\}
\end{aligned}
$$

O resultado da estratificação, utilizando uma função $\gamma_{\text {freq }}$ aplicado ao conjunto resíduo seria:

$$
\begin{aligned}
\gamma_{\text {freq }}(\mathcal{R})=\{ & \{\{A \sqsubseteq B\},\{B \sqsubseteq C, B \sqsubseteq E\}\}, \\
& \{\{A \sqsubseteq B\},\{B \sqsubseteq C, E \sqsubseteq D\}\}, \\
& \{\{A \sqsubseteq B\},\{C \sqsubseteq D, B \sqsubseteq E\}\}, \\
& \{\{A \sqsubseteq B\},\{C \sqsubseteq D, E \sqsubseteq D\}\}, \\
& \{\{B \sqsubseteq C, C \sqsubseteq D, B \sqsubseteq E, E \sqsubseteq D\}\}\}
\end{aligned}
$$

Seguindo o princípio da majoração, a opinião mais votada pela audiência será a escolhida. Nesse caso, nossa função de seleção seleciona os conjuntos $\mathcal{S}_{1}=\{\{A \sqsubseteq B\},\{B \sqsubseteq C, B \sqsubseteq E\}\}$, $\mathcal{S}_{2}=\{\{A \sqsubseteq B\},\{B \sqsubseteq C, E \sqsubseteq D\}\}, \mathcal{S}_{3}=\{\{A \sqsubseteq B\},\{C \sqsubseteq D, B \sqsubseteq E\}\}, \mathcal{S}_{4}=\{\{A \sqsubseteq B\},\{C \sqsubseteq$ $D, E \sqsubseteq D\}\}$. A seleção desses conjuntos representa o voto da maioria pelo axioma $A \sqsubseteq B$, que aparece em 4 de 5 elementos do conjunto resíduo.

O resultado da função de seleção utilizada no Exemplo 3.9 foi surpreendente no sentido de que os elementos do conjunto resíduo escolhidos como prioritários possuem, cada um 3 axiomas, 
ao passo que o único conjunto deixado possuía 4 axiomas. Isso se dá justamente por conta do caráter de majoração do operador de estratificação utilizado. Em comparação ao comportamento de majoração, o comportamento de arbitragem busca minimizar a insatisfação individual. Um operador que possuísse essa natureza de arbitragem poderia escolher o elemento deixado de fora no exemplo. Um exemplo desse tipo de operador é dado na próxima seção.

\subsubsection{Seleção dos Maiores Itens no Conjunto Resíduo}

Um operador com a natureza de arbitragem garante que a escolha pela solução não depende do número de repetições entre as possíveis soluções, esse tipo de operador utiliza a filosofia da minimização da insatisfação individual, i.e., a melhor solução apontada pelo operador pode não ser a mais recorrente entre os elementos do conjunto resíduo.

Essa estratégia de estratificação possui implementação trivial. O objetivo aqui é selecionar o item dentro do conjunto resíduo que tenha o maior número de axiomas com a intenção de manter a maior quantidade de informação possível, em detrimento da confiabilidade dessa informação.

Vale a pena notar que essa forma de estratificação cria um único estrato por item do conjunto resíduo. A função de seleção recupera então o maior desses estratos. O exemplo 3.10 mostra o funcionamento dessa estratégia.

Exemplo 3.10 (continuação do Exemplo 3.9.) Suponhamos que no lugar do operador $\gamma_{\text {freq }}$ utilizemos o $\gamma_{\text {maior }}$ em $\mathcal{R}=\mathcal{O} \perp(A \sqsubseteq D)$, obteríamos então:

$$
\begin{aligned}
\gamma_{\text {maior }}(\mathcal{R})=\{ & \{A \sqsubseteq B, B \sqsubseteq C, B \sqsubseteq E\}, \\
& \{A \sqsubseteq B, B \sqsubseteq C, E \sqsubseteq D\}, \\
& \{A \sqsubseteq B, C \sqsubseteq D, B \sqsubseteq E\}, \\
& \{A \sqsubseteq B, C \sqsubseteq D, E \sqsubseteq D\}, \\
& \{B \sqsubseteq C, C \sqsubseteq D, B \sqsubseteq E, E \sqsubseteq D\}\}
\end{aligned}
$$

Seguindo o princípio da arbitragem, selecionaremos que minimize a insatisfação individual, i.e., o item do conjunto resíduo que mantenha a maior quantidade de informação $\mathcal{S}_{5}=\{B \sqsubseteq C, C \sqsubseteq$ $D, B \sqsubseteq E, E \sqsubseteq D\}$.

\subsubsection{Relevância Semântica}

A tradução da função da técnica de estratificação que prioriza a relevância semântica, utilizada na depuração de granularidade fina, tem como objetivo manter o conjunto de informações a partir do qual o maior número de inferências pode ser realizado. A natureza desse operador é a Arbitragem, uma vez que a escolha do grupo não interfere na escolha da melhor solução.

O algoritmo que implementa essa função de estratificação atribui notas para cada um dos $\mathcal{R}_{i}$ itens no conjunto resíduo. Essas notas são calculadas a partir do número de elementos que obtemos ao calcularmos o conjunto de inferências não triviais que podem ser realizadas a partir de $\mathcal{R}_{i}$, i.e. $\left|C n\left(\mathcal{R}_{i}\right)\right|$. Em seguida, selecionamos o item $\mathcal{R}_{i}$ que possui o maior número de inferências não triviais.

O funcionamento desse operador de estratificação é ilustrado em uma continuação do Exemplo 3.9, no Exemplo 3.11. 
Exemplo 3.11 (continuação do Exemplo 3.9) Suponha que utilizemos uma função de estra-

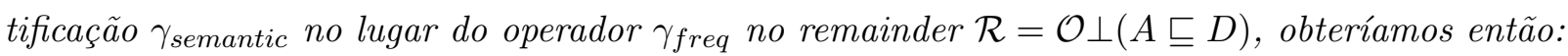

$$
\gamma_{\text {semantic }}(\mathcal{R})=\{2,1,0,0,1\}
$$

$O$ cálculo de $\gamma_{\text {semantic }}$ foi realizado da seguinte forma, lembrando que os elementos no conjunto $\mathcal{R}$ são: $\mathcal{R}_{1}=A \sqsubseteq B, B \sqsubseteq C, B \sqsubseteq E, \mathcal{R}_{2}=A \sqsubseteq B, B \sqsubseteq C, E \sqsubseteq D, \mathcal{R}_{3}=A \sqsubseteq B, C \sqsubseteq D, B \sqsubseteq E$, $\mathcal{R}_{4}=A \sqsubseteq B, C \sqsubseteq D, E \sqsubseteq D$ e $\mathcal{R}_{5}=B \sqsubseteq C, C \sqsubseteq D, B \sqsubseteq E, E \sqsubseteq D$. A função $\gamma_{\text {semantic precisa }}$ então calcular as consequências lógicas para cada um dos $\mathcal{R}_{i}$ elementos em $\mathcal{R}$, obtendo: $C n\left(\mathcal{R}_{1}\right)=$ $\{A \sqsubseteq C, A \sqsubseteq E\}$, com tamanho 2; $C n\left(\mathcal{R}_{2}\right)=\{A \sqsubseteq C\}$, com tamanho $1 ; C n\left(\mathcal{R}_{3}\right)=\{A \sqsubseteq E\}$, com tamanho 1 e $C n\left(\mathcal{R}_{4}\right)=\{C \sqcup E \sqsubseteq D\}$ com tamanho 1; e $C n\left(\mathcal{R}_{5}\right)=\{B \sqsubseteq D\}$, com tamanho 1 .

Assim, o algoritmo escolheria $\mathcal{R}_{1}$ como a solução para a seleção.

Uma variação desse algoritmo que tivesse natureza de Majoração poderia selecionar elementos cujas inferências são mais frequentes no conjunto resíduo. No Exemplo 3.11, o conjunto escolhido seria o $\mathcal{R}_{1}$, pois seus elementos são "apoiados" por $\mathcal{R}_{2}$ e $\mathcal{R}_{3}$.

\subsubsection{Relevância Sintática}

No contexto da construção do conjunto kernel, a função de incisão baseada em relevância sintática promove a remoção de axiomas mais desconexos (da ontologia inicial) presentes nos elementos do conjunto kernel. O objetivo é então, a remoção de axiomas cujos conceitos estejam menos ligados ao resto da ontologia. Com isso, esperamos que os axiomas removidos sejam menos importantes para a ontologia.

A tradução dessa técnica para a construção de funções de seleção busca realizar o mesmo - escolher soluções que sejam mais conectadas entre todos os elementos do conjunto resíduo. O algoritmo funciona da seguinte forma: para cada axioma é atribuído um contador, chamado índice de conectividade sintática que relaciona os conceitos contidos nesse axioma e a presença desses conceitos em outros axiomas assim como definido na Seção 2.1.1.1. Os estratos mais altos possuem axiomas cujos conceitos são mais fortemente conectados entre si. A função de seleção então seleciona o conjunto que possuir mais elementos em seus estratos mais altos.

O Exemplo 3.12 ilustra o funcionamento do algoritmo:

Exemplo 3.12 (Continuação do Exemplo 3.7) Considere a ontologia:

$$
\begin{aligned}
\mathcal{O}=\{A & \sqsubseteq B \\
B & \sqsubseteq C \\
C & \sqsubseteq E \\
A & \sqsubseteq F \\
& F \sqsubseteq E\} \models A \sqsubseteq E
\end{aligned}
$$


Considerando $\alpha=A \sqsubseteq E$, calculando o conjunto resíduo $\mathcal{R}=\mathcal{O} \perp \alpha$ obtemos:

$$
\begin{aligned}
\mathcal{O} \perp(A \sqsubseteq E)=\{ & \{A \sqsubseteq B, B \sqsubseteq C, A \sqsubseteq F\}, \\
& \{A \sqsubseteq B, B \sqsubseteq C, F \sqsubseteq E\}, \\
& \{A \sqsubseteq B, C \sqsubseteq E, A \sqsubseteq F\}, \\
& \{A \sqsubseteq B, C \sqsubseteq E, F \sqsubseteq E\}, \\
& \{B \sqsubseteq C, C \sqsubseteq E, A \sqsubseteq F\}, \\
& \{B \sqsubseteq C, C \sqsubseteq E, F \sqsubseteq E\}\}
\end{aligned}
$$

Utilizando a função de estratificação $\gamma_{\text {syntactic }}$ obtemos:

$$
\begin{aligned}
\gamma_{\text {syntactic }}(\mathcal{R})=\{ & \{<\{A \sqsubseteq B\}, 2>,<\{A \sqsubseteq F, B \sqsubseteq C\}, 1>\}, \\
& \{<\{A \sqsubseteq B, B \sqsubseteq C\}, 1>,<\{F \sqsubseteq E\}, 0>\}, \\
& \{<\{A \sqsubseteq B, A \sqsubseteq F\}, 1>,<\{C \sqsubseteq E\}, 0>\}, \\
& \{<\{C \sqsubseteq E, F \sqsubseteq E\}, 1>,<\{A \sqsubseteq B\}, 0>\}, \\
& \{<\{B \sqsubseteq C, C \sqsubseteq E\}, 1>,<\{A \sqsubseteq F\}, 0>\}, \\
& \{<\{C \sqsubseteq E\}, 2>,<\{B \sqsubseteq C, F \sqsubseteq E\}, 1>\}\}
\end{aligned}
$$

Aqui, cada estrato é representado por uma dupla, onde a primeira posição corresponde ao conjunto de axiomas presentes no estrato e a segunda posição corresponde ao índice de conectividade sintática. A escolha então é feita pelos conjuntos que possuem maior índice de conectividade sintática, i.e., $\mathcal{S}_{1}=\{<\{A \sqsubseteq B\}, 2>,<\{A \sqsubseteq F, B \sqsubseteq C\}, 1>\}$ e $\mathcal{S}_{6}=\{<\{C \sqsubseteq E\}, 2>,<$ $\{B \sqsubseteq C, F \sqsubseteq E\}, 1>\}\}$. A escolha por um dos dois fica a critério do projetista, podendo utilizar uma segunda estratégia de estratificação para escolher entre os dois ou manter os dois e utilizar a interseção como solução.

Esse tipo de estratégia também está mais alinhada com a ideia de operadores de fusão baseados em Arbitragem, uma vez que não é necessário levar em conta a estrutura de todos os estratos para escolher o item do conjunto que será utilizado como solução para a remoção da inferência indesejada.

\subsection{Depuração de Granularidade Grossa Parcial}

O processo para fusão de ontologias que parte da depuração com granularidade grossa, levando em conta um único elemento pertencente ao conjunto resíduo pode ser visto na Figura 3.4.

Essa forma de depuração dispensa o uso de uma função de estratificação, pelo menos de forma explicita. O objetivo aqui é escolher o melhor dos itens do conjunto resíduo que se encaixe nos requisitos de depuração do usuário.

Como visto no Capítulo 2, o algoritmo de cálculo de elementos do conjunto resíduo pode ser visto como o dual do algoritmo para construção de elementos do conjunto kernel, i.e., uma estratégia de encolhe-expande, onde os elementos são primeiro removidos até que a inferência indesejada não seja mais válida, em seguida readicionando-os de modo que possamos garantir a maximalidade. 
A injeção da função de estratificação no algoritmo Black-box para cálculo de elementos do conjunto resíduo se dá a partir das estratégias de encolhimento e expansão do algoritmo. Tipicamente o que deve ser realizado é uma busca pelo elemento que pior atenda a um determinado critério de estratificação, e.g., o axioma menos conectado estruturalmente (relevância sintática), em seguida devemos realizar o encolhimento preferindo esses elementos. Durante a expansão devemos realizar a reinserção dos elementos removidos, o que deve ser feito na ordem reversa do encolhimento.

\subsubsection{Construção Guiada de Um Elemento do Conjunto Resíduo}

Nessa seção apresentamos como algumas ideias clássicas de estratificação podem ser utilizadas na construção de estratégias de encolhimento/expansão, guiando a construção do conjunto resíduo.

O princípio aplicado para a utilização das estratégia de priorização de axiomas mais gerais [QP07], relevância semântica [Kal06] e relevância sintática [Kal06] é simples. Utilizamos uma função de seleção que é invocada antes da tomada de decisão de qual axioma será utilizado tanto para o encolhimento quanto para a expansão. Essa função receberá o conjunto de todos os elementos restantes na ontologia - ou que já foram removidos ou que precisam ser readicionados - e retorna o elemento mais promissor para a função de cálculo do conjunto resíduo.

Os operadores de estratificação por frequência e o operador de seleção de maiores itens no conjunto resíduo não podem ser implementados de forma guiada, uma vez que o seu cálculo para ser apurado precisa que todo o conjunto esteja disponível. Portanto, somente as três formas: seleção baseada na generalidade de axiomas, relevância semântica e relevância sintática estão disponíveis no nosso processo, quando o usuário decide construir apenas um elemento do conjunto resíduo.

Generalidade de Axiomas O algoritmo utilizado para a construção de um elemento que possua os axiomas mais gerais possível possui uma ideia de implementação simples: criamos uma estratégia de encolhimento que selecione os elementos mais gerais possíveis. Assim, de forma iterativa, os elementos mais específicos são removidos da base. Em seguida, durante a fase de expansão, adaptamos a estratégia para que essa readicione os elementos mais gerais primeiro, i.e., readicione na ordem inversa na qual eles foram removidos da base, e.g., utilizando uma pilha.

Relevância Semântica Para utilizar a ideia da estratificação baseada na relevância semântica de um axioma precisamos realizar uma série de checagens que contam o número de inferências que são perdidas caso cada um dos axiomas seja removido. Tendo essa ordem de importância em mãos, podemos fazer o encolhimento selecionando os elementos cuja remoção impactem de forma menos negativa no número de inferências válidas para a base. A expansão se dá, assim como no caso da Generalidade de Axiomas, readicionando os axiomas mais gerais, i.e., a ordem reversa do encolhimento.

O desempenho nesse caso terá um impacto negativo, uma vez que é necessário realizar a ordenação da base inteira em termos de inferências perdidas, sendo necessário pelo menos $O(n \log n)$ chamadas ao mecanismo de inferência para ordenar os axiomas por quantidade de inferências.

Relevância Sintática A adaptação dessa estratégia para a construção de um único elemento do conjunto resíduo tem como objetivo, durante o encolhimento, a cada passo remover o axioma menos conectado aos outros axiomas da ontologia. Assim como na relevância semântica, é necessário 


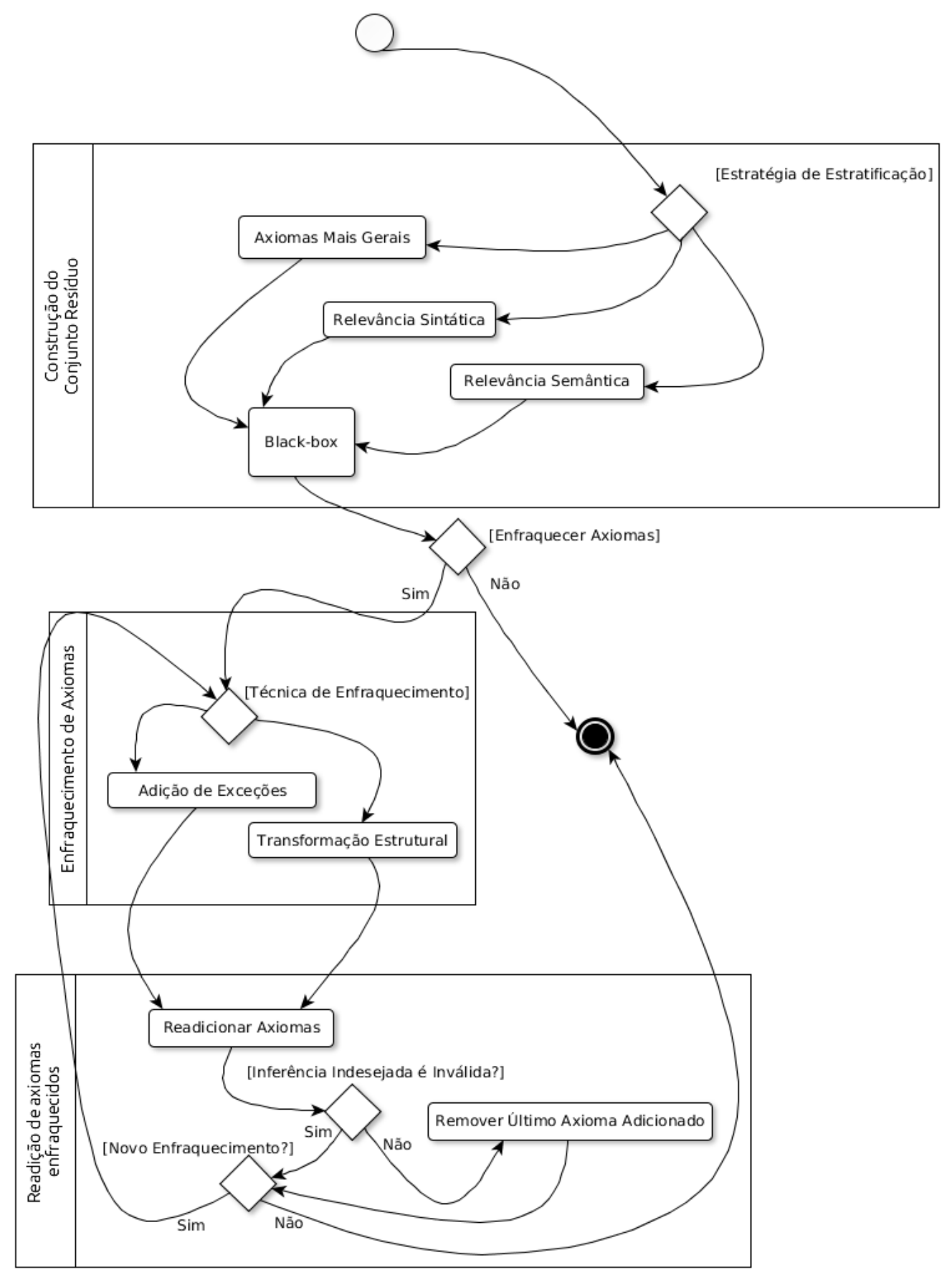

Figura 3.4: Processo de fusão proposto para o caso de depuração de granularidade grossa com o conjunto resíduo parcial 
ordenar axiomas de acordo com as suas conectividades. Mais do que isso, é necessário agrupar axiomas por índice de conectividade sintática, o mesmo algoritmo da estratificação comum, apresentado na Seção 3.3.2.5. Após a formação desses agrupamentos, o encolhimento deve começar pelos menores agrupamentos, seguindo para os agrupamentos maiores. A expansão que se segue deve então readicionar elementos que se encontram em agrupamentos maiores, seguindo em direção aos menores.

O desempenho mais uma vez sofre impacto, ao utilizar essa abordagem pois agora temos que realizar o agrupamento de axiomas por nível de conexão. Esse agrupamento pode ser realizado, em $\mathcal{O}\left(n^{2}\right)$, uma vez que precisamos checar a conexão entre todos os elementos entre si. Esse resultado pode ser melhorado se considerarmos que a base de crenças já guarda essa informação, como o proposto por Wassermann em [Was01].

Após a fase de depuração, temos construído o conjunto kernel, ou um elemento do conjunto resíduo obtido via estratificação aplicada ao conjunto resíduo completo, ou obtido via construção guiada.

A essa altura o processo prevê o uso de alguma estratégia de enfraquecimento para tentar remover elementos no kernel, com informações irrelevantes para a inferência indesejada, ou adicionar elementos ao elemento do conjunto resíduo escolhido.

\subsection{Enfraquecimento de Axiomas}

Durante essa fase no nosso processo, o usuário é apresentado a opções de modificações nos axiomas, de forma que a exclusão não seja a única solução. Nessa seção vamos discutir o papel desempenhado pelo enfraquecimento de axiomas no processo de remoção de inferências indesejadas.

Primeiramente vamos discutir alguns conceitos necessários para o entendimento do processo de enfraquecimento, em seguida vamos apresentar um conjunto comum de técnicas encontradas na literatura para a construção de versões enfraquecidas de axiomas.

\subsubsection{Definições}

Em [QLB06, MLB05], Qi et al. e Meyer et al. propuseram técnicas que realizam, de forma iterativa, o enfraquecimento de um determinado axioma. O objetivo dessa fase é tentar manter o maior número de axiomas, modificando os que se encontram envolvidos em conflitos, deixando-os um pouco menos restritivos. O Exemplo 3.13 ilustra esse conceito.

Definição 3.1 (Enfraquecimento de Axiomas [Hor11].) Um axioma $\alpha_{1}$ é considerado mais fraco do que $\alpha_{2}$ se e somente se $\alpha_{2}=\alpha_{1}$ e $\alpha_{1} \not \models \alpha_{2}$.

Exemplo 3.13 $A \sqsubseteq B$ é mais fraco que $A \sqsubseteq B \sqcap C$, uma vez que $A \sqsubseteq B \sqcap C \models A \sqsubseteq B$, porém $A \sqsubseteq B \not \models A \sqsubseteq B \sqcap C$.

\subsubsection{Axiomas de Granularidade Fina}

Em muitos casos, os axiomas possuem ainda internamente partes que não são relevantes para a explicação da inferência indesejada. Uma área relacionada, que também tem como objetivo a construção de axiomas pequenos o suficiente de forma que a remoção da informação seja mínima é a construção de justificativas de granularidade fina. 
Assim como para a área de resolução de conflitos, para a obtenção de justificativas também é primordial que somente partes tidas como relevantes sejam mantidas, até mesmo para fins de depuração de ontologias. O Exemplo 3.14 mostra uma forma de construção de justificativas e como as mesmas sofrem do problema de axiomas com partes irrelevantes para a inferência indesejada.

Exemplo 3.14 Considere a ontologia:

$$
\begin{aligned}
\mathcal{O}=\{A \sqsubseteq C \sqcap D, & \\
& B \sqcup E \sqsubseteq A, \\
& B \sqsubseteq F, \\
& F \sqsubseteq A\} \models B \sqsubseteq C
\end{aligned}
$$

Se realizarmos o cálculo do conjunto kernel (ou justificativas), considerando $\alpha=B \sqsubseteq C$ obtemos:

$$
\mathcal{J}_{1}=\{A \sqsubseteq C \sqcap D, B \sqcup E \sqsubseteq A\}
$$

$e$

$$
\mathcal{J}_{2}=\{B \sqsubseteq F, F \sqsubseteq A, A \sqsubseteq C \sqcap D\}
$$

É fácil de ver que em $\mathcal{J}_{1}$, os axiomas $A \sqsubseteq C \sqcap D$ e $B \sqcup E \sqsubseteq A$ possuem partes que não são relevantes para a tarefa de derivação de $B \sqsubseteq C$, no primeiro a informação $\sqcap D$ e no segundo a informação $\sqcup E$.

Assim, para resolver o problema de descartar trechos de axiomas que são irrelevantes para a inferência indesejada, temos que construir versões menores, i.e., enfraquecidas dos axiomas originais, de forma que somente o que é pertinente a inferência indesejada seja descartado.

Na literatura, existem basicamente duas abordagens bastante similares que propõem a utilização de axiomas de granularidade fina na construção de justificativas como abordagem para resolver o problema de descartar informações irrelevantes ao conflito. Kalyanpur, em [Kal06], constrói um serviço para a depuração de ontologias e lança a ideia de que algumas vezes, o axioma descartado durante a depuração possui informações que não são pertinentes a depuração, ou seja, são dispensáveis para o cálculo de justificativas.

Horridge [Hor11] se preocupa com um outro fenômeno causado por axiomas de granularidade grossa, o mascaramento de justificativas. Nesse fenômeno, justificativas alternativas a consequências lógicas são mascaradas por outras. É importante notar que esse fenômeno não está restrito apenas ao cálculo de justificativas para consequências lógicas, mas também na construção de conjuntos kernel. Em [Hor11], Horridge cita cinco formas básicas de mascaramento e provê uma caracterização formal para elas. As cinco formas são:

Mascaramento Interno Esse tipo de problema ocorre quando uma justificativa possui, internamente, múltiplas razões para uma determinada consequência lógica. Por exemplo, considere a ontologia

$$
\mathcal{O}=\{A \sqsubseteq B \sqcap \neg B \sqcap C \sqcap \neg C\}
$$

$\mathcal{O}$ possui dois conjuntos de axiomas que podem ser utilizados para justificar que $A \sqsubseteq \perp: \mathcal{J}_{1}=$ $\{A \sqsubseteq B \sqcap \neg B\}$ e $\mathcal{J}_{2}=\{A \sqsubseteq C \sqcap \neg C\}$. 
Mascaramento Cruzado Esse tipo de problema ocorre quando partes de axiomas se combinam para formar novas justificativas, e.g., a ontologia

$$
\mathcal{O}=\{A \sqsubseteq B \sqcap \neg B \sqcap C, A \sqsubseteq D \sqcap \neg D \sqcap \neg C\}
$$

possui dois conjuntos de axiomas utilizados como justificativa para $A \sqsubseteq \perp: \mathcal{J}_{1}=\{A \sqsubseteq B \sqcap \neg B \sqcap C\}$ e $\mathcal{J}_{2}=\{A \sqsubseteq D \sqcap \neg D \sqcap \neg C\}$. Porém, a união dessas duas justificativas pode produzir um novo motivo para o conflito, $\mathcal{J}_{3}=\{A \sqsubseteq C \sqcap \neg C\}$.

Mascaramento Externo Esse tipo de problema ocorre quando axiomas externos às justificativas podem ser incorporados a estas para a obtenção de novas justificativas, e.g. a ontologia

$$
\mathcal{O}=\{A \sqsubseteq B \sqcap \neg B \sqcap C, A \sqsubseteq \neg C\}
$$

possui uma justificativa para $A \sqsubseteq \perp: \mathcal{J}_{1}=\{A \sqsubseteq B \sqcap \neg B \sqcap C\}$. No entanto, se considerarmos o axioma $A \sqsubseteq \neg C$, veremos que esse também pode ser utilizado na construção de uma nova justificativa, assim, intuitivamente, a ontologia $\mathcal{O}$ possui duas justificativas para $A \sqsubseteq \perp$ : $\mathcal{J}_{1}=$ $\{A \sqsubseteq B \sqcap \neg B\}$ e $\mathcal{J}_{2}=\{A \sqsubseteq C, A \sqsubseteq \neg C\}$.

Mascaramento de Núcleo Compartilhado Esse tipo de problema ocorre quando em duas justificativas, após a remoção de partes supérfluas se tornam a mesma justificativa, e.g., a ontologia

$$
\mathcal{O}=\{A \sqsubseteq B \sqcap \neg B \sqcap C, A \sqsubseteq B \sqcap \neg B\}
$$

possui duas justificativas para $A \sqsubseteq \perp: \mathcal{J}_{1}=\{A \sqsubseteq B \sqcap \neg B \sqcap C\}$ e $\mathcal{J}_{2}=\{A \sqsubseteq B \sqcap \neg B\}$. Ao examinarmos a justificativa $\mathcal{J}_{1}$, ao removermos a parte supérflua: $\sqcap C, \mathcal{J}_{1}$ se torna equivalente a $\mathcal{J}_{2}$.

Mascaramento devido ao enfraquecimento Esse fenômeno ocorre quando versões mais fracas de um axioma podem ser utilizadas para obter novas justificativas, e.g. a ontologia

$$
\mathcal{O}=\{A \sqsubseteq \geq 2 \text { R.C, } A \sqsubseteq \geq 1 \text { R.D, } C \sqsubseteq \neg D, A \sqsubseteq \leq 1 R\}
$$

possui uma única justificativa para $A \sqsubseteq \perp: \mathcal{J}_{1}=\{A \sqsubseteq \geq 2 R . C, A \sqsubseteq \leq 1 R\}$, porém uma nova justificativa pode ser obtida se examinarmos a versão enfraquecida do axioma $\phi=A \sqsubseteq \geq 2 R . C$, o axioma $\phi^{\prime}=A \sqsubseteq \geq 1$ R.C. Com esse axioma construímos a justificativa $\mathcal{J}_{2}=\{A \sqsubseteq \geq 1 R . C$, $A \sqsubseteq \geq 1 R . D, C \sqsubseteq \neg D, A \sqsubseteq \leq 1 R\}$. Sendo essa forma de mascaramento possível de ocorrer dentro de todos os anteriores.

\subsubsection{Calculando Justificativas de Granulação Fina}

Os trabalhos de Horridge [Hor11] e Kalyanpur [Kal06] citam, como peça fundamental para a obtenção de justificativas tidas como de granularidade fina, a existência de uma transformação estrutural responsável por construir uma extensão conservativa de um conjunto de axiomas. Em outras palavras, seja a ontologia $\mathcal{O}^{\prime}$ obtida a partir de uma transformação estrutural $\Delta$ e seja um modelo $\mathcal{M}$, se $\mathcal{M} \models \mathcal{O}^{\prime}$, então $\mathcal{M} \models \mathcal{O}$. Essa função de transformação estrutural é responsável por 
construir versões enfraquecidas e menores dos axiomas, nos quais todas as partes contribuam com a justificativa.

Ambos autores definem então o conceito de justificativas precisas, justificativas que possuem axiomas que contêm somente conceitos indispensáveis para uma derivação, i.e., as partes que compõem os axiomas presentes em uma justificativa precisa, caso removidas resultam na quebra da derivação de um axioma, e.g., suponha a ontologia $\mathcal{O}=\{A \sqsubseteq B, B \sqsubseteq C \sqcap F, C \sqsubseteq D\}$, de acordo com a relação de consequência lógica clássica para lógicas de descrição, podemos estabelecer que $\mathcal{O} \mid=A \sqsubseteq D$. Caso o usuário não queira que seja possível derivar tal informação algum dos axiomas pertencentes a justificativa $\mathcal{J}=\{A \sqsubseteq B, B \sqsubseteq C \sqcap F, C \sqsubseteq D\}$ deve ser removido. Porém, é possível observar que o axioma $B \sqsubseteq C \sqcap F$ contém uma informação que não contribui em nada na derivação $\mathcal{O} \models A \sqsubseteq D$, a informação $B \sqsubseteq F$, assim sendo a justificativa $\mathcal{J}$ não pode ser considerada precisa.

Seguindo essa cadeia de raciocínio, é possível estabelecer uma propriedade de superfluidade nos axiomas, no que diz respeito a uma dada justificativa. Uma parte do axioma pode ser considerada supérflua, não colaborando em nada, na construção da justificativa para uma consequência lógica.

Horridge em [Hor11] elabora uma definição formal de superfluidade, que ocorre quando em um dado axioma, algum de seus conceitos pode ser substituído por $T$, no caso de se encontrar a direita da subsunção e por $\perp$, no caso de se encontrar a esquerda da subsunção. Por exemplo, o axioma $\phi=A \sqsubseteq C \sqcap D$ pode ser considerado uma justificativa para $A \sqsubseteq C$, porém, esse contém informação que pode ser considerada de forma intuitiva, supérflua. No caso a informação implícita $A \sqsubseteq D$. Utilizando a definição de Horridge, podemos reescrever o axioma $\phi$ como $A \sqsubseteq C \sqcap \top$, sem que o mesmo deixe de ser justificativa para $A \sqsubseteq D$. De forma análoga, o axioma $\phi^{\prime}=A \sqcup B \sqsubseteq C$ pode ser rescrito como $A \sqcup \perp \sqsubseteq C$ não sendo alterada a relação de justificativa para $A \sqsubseteq C$.

O processo de construção de justificativas precisas apresentado por Kalyanpur em [Kal06] possui um carácter muito mais algorítmico. O mesmo propõe uma função para a divisão de conjuntos de axiomas e define como precisa, toda justificativa que seja obtida por uma versão dividida da ontologia, i.e., uma versão modificada, construída a partir da função split () por ele proposta. Mais detalhes sobre as regras da transformação em [Kal06].

\subsubsection{Transformação Estrutural}

Como já foi introduzido na seção anterior o papel desempenhado pela transformação estrutural é o de reescrever axiomas de forma que sejam geradas versões mais fracas e menores possíveis. Horridge, em [Hor11] descreve de forma intuitiva esse processo como sendo a reescrita de axiomas de modo que uma justificativa contenha os axiomas de granularidade fina e enfraquecidos de forma que uma versão de granularidade mais fina ou contendo axiomas mais enfraquecidos quebre a justificativa. Kalyanpur [Kal06] entretanto, não provê essas definições do que seriam justificativas de granularidade fina.

Ambos autores definem formas de transformação que são aplicadas a justificativas, produzindo versões de granularidade fina.

Horridge [Hor11] faz uso da transformação estrutural $\delta$ proposta por Plaisted e Greenbaum em [PG86], que é centrada na idéia de criação de novos conceitos para evitar o fenômeno da explosão, ao tentar transformar fórmulas em lógica proposicional em uma forma normal clausal. A transformação 
$\delta$ recebe como entrada um conjunto de axiomas $\mathcal{S}$ e constrói uma extensão conservativa desse conjunto, $\mathcal{S}^{\prime}$ que embora não seja equivalente a $\mathcal{S}$ é equi-consistente e equi-satisfazível.

Kalyanpur constrói uma ideia de divisão de um conjunto de fórmulas - função chamada split ( ) - que também é capaz de construir uma extensão conservativa equi-consistente e equi-satisfazível. A função split () também tem a capacidade de criar novos conceitos, não com a justificativa de evitar a explosão exponencial no número de conceitos mas como forma de reduzir o tamanho de axiomas que não poderiam ser reescritos de outra forma, e.g., o axioma $C \sqsubseteq \exists P$. $(A \sqcap B)$ que não pode ser rescrito como $\{C \sqsubseteq \exists P . A, C \sqsubseteq \exists P . B\}$ pode ser reescrito como $\{C \sqsubseteq \exists P . E, E \sqsubseteq A \sqcap B\}$, onde $E$ é um conceito novo, não pertencente ao conjunto original. Detalhes sobre a transformação $\delta$ podem ser encontrados em [Hor11].

\subsubsection{Transformação $\delta$ Versus Função split()}

Para o nosso processo foi importante realizar uma comparação entre as duas abordagens e mesmo que bastante similares, apresentam características particulares que podem auxiliar no processo de restauração de conflitos.

O trabalho de Horridge [Hor11] é mais centrado na questão da construção de justificativas de granularidade fina. Nesse trabalho o autor caracteriza, em um nível formal, um conjunto de fenômenos decorrentes da utilização de axiomas de granularidade grossa, os chamados mascaramentos. Para resolver esses problemas, a função $\delta$ foi proposta. Porém, Kalyanpur em [Kal06] também define uma função de características similares e objetivos próximos no que diz respeito a construção de axiomas que possuem somente partes pertinentes as justificativas.

Nesse ponto o trabalho de Horridge mostra um outro aspecto importante, o enfraquecimento de axiomas como critério para a construção de justificativas precisas. Esse ponto é importante para que seja evitado o fenômeno de mascaramento devido ao enfraquecimento de axiomas.

Ao compararmos as duas funções de transformação, chegamos a conclusão que ambas, no geral produzem a mesma saída para as regras de transformação com a diferença que sempre que Kalyanpur adiciona um novo conceito, ele não se preocupa em produzir a versão mais enfraquecida do mesmo, ele introduz uma versão exata. Por exemplo, para enfraquecer o axioma $\phi=A \sqsubseteq \exists R .(C \sqcap D)$, Kalyanpur propõe a introdução de um novo conceito, $E$, que deve substituir o que se encontra a direita da propriedade $R$ no axioma $\phi$, produzindo um axioma $\phi^{\prime}=A \sqsubseteq \exists R$.E. Em adição, a função split () adiciona os axiomas: $E \sqsubseteq C \sqcap D$ e $(C \sqcap D) \sqsubseteq E$.

De forma análoga, Horridge também propõe a adição de um novo conceito, porém adiciona um conjunto enfraquecido de axiomas. A utilização da função $\delta$ tem como resultado o axioma $\phi^{\prime}=A \sqsubseteq \exists R . E$ e um único novo axioma, $E \sqsubseteq C \sqcap D$, que é uma versão enfraquecida do proposto por Kalyanpur.

Dois pontos devem ser considerados nessa comparação: a importância de se evitar a ocorrência de mascaramentos por enfraquecimento e a possibilidade de resolução de conflitos ao produzirmos uma versão mais enfraquecida de um axioma. Tendo esses dois pontos em mente, a escolha pela abordagem proposta por Horridge parece ser mais natural.

Em nosso processo, fazemos uso da função $\delta$ para a construção de justificativas (ou conjuntos kernel) de granularidade fina, uma vez que essa possui as mesmas características da função split (), porém possui implementação já consolidada ${ }^{1}$.

\footnotetext{
${ }^{1}$ https://github.com/matthewhorridge/owlexplanation
} 


\subsubsection{Adição de Exceções}

Uma das formas mais básicas de realizar o enfraquecimento, é a técnica chamada Adição de Exceções [QLB06], onde axiomas são modificados de forma que deixem explicito que determinados indivíduos não são afetados pelos mesmos. O processo de adição de exceções a axiomas de subordinação parte do princípio que a existência de determinados indivíduos é responsável pela existência de conflitos. Por motivos óbvios, Qi et al., em [QLB06], decidiram somente tratar de casos de inconsistências.

Uma solução algorítmica para a adição de exceções utilizando uma abordagem força bruta é simples de projetar. A rotina deve partir dos conceitos não satisfazíveis e analisar o conjunto de indivíduos que pertencem a tal conceito. O algoritmo deve testar todos os possíveis sub-conjuntos de indivíduos.

Como resultado dessa adição de exceções obtemos a substituição de cada axioma $C \sqsubseteq D$ envolvido em um conflito, pelo axioma $\left(C \sqcap \neg\left\{a_{1}\right\} \sqcap \neg\left\{a_{2}\right\} \sqcap \ldots \sqcap \neg\left\{a_{n}\right\}\right) \sqsubseteq D$, onde $n$ é a quantidade de exceções necessárias para resolver o conflito. O problema com essa abordagem está na perda em poder do axioma. Ao definir uma exceção, se faz necessário que um novo conjunto de axiomas seja adicionado, deixando claro que o indivíduo sob o qual a exceção vigora é diferente de todos os demais na ontologia. O Exemplo 3.15 ilustra o funcionamento do mecanismo de adição de exceções a axiomas de subordinação.

Exemplo 3.15 Suponha que tenhamos a ontologia:

$$
\begin{aligned}
\mathcal{O}= & \{\text { passaro }(\text { tweety }), \\
& \neg \text { voa }(\text { tweety }), \\
& \text { passaro }(\text { chirpy }), \\
& \text { passaro } \sqsubseteq \text { voa }\} \models \top \sqsubseteq \perp
\end{aligned}
$$

O único axioma de subordinação nessa ontologia é passaro $\sqsubseteq$ voa, que diz que todo pássaro voa. A ontologia se encontra inconsistente pois temos o indivíduo tweety que é um pássaro e não voa. A adição de exceções utilizando abordagem de Qi et al.[QLB06] produziria a ontologia

$$
\begin{aligned}
& \mathcal{O}^{\prime}=\{\text { passaro }(\text { tweety }), \\
& \neg \text { voa }(\text { tweety }), \\
& \text { passaro }(\text { chirpy }), \\
&(\text { passaro } \sqcap \neg\{\text { tweety }\}) \sqsubseteq v o a\}
\end{aligned}
$$

O problema com o resultado apresentado no Exemplo 3.15 é que a partir de $\mathcal{O}^{\prime}$ não é mais possível inferir que chirpy também é capaz de voar, i.e. $\{$ chirpy\} $\sqsubseteq$ voa. Para resolver o problema temos que deixar explícito que chirpy não é o mesmo indivíduo que tweety, i.e. adicionar o axioma chirpy $\neq$ tweety a $\mathcal{O}^{\prime}$. Um algoritmo que automatiza a inserção de axiomas de desigualdade é simples de ser implementada. A dificuldade maior está em manter controle sobre a inserção de novos indivíduos na ontologia, ou seja, definir uma forma de assegurar que para cada novo indivíduo inserido na ontologia, um axioma que diz que esse indivíduo é diferente de indivíduos em exceções também seja inserido. 
Como açúcar sintático, também é possível incluir exceções em axiomas de disjunção, por esses muitas vezes serem os responsáveis pela introdução de conflitos. O algoritmo para fazer essa adição é idêntico ao que adiciona exceções a axiomas de subordinação apresentado anteriormente uma vez que axiomas de disjunção podem ser rescritos como axiomas comuns de subordinação, e.g., o axioma $A$ DisjointWith $B$ pode ser rescrito como $A \sqsubseteq \neg B$. Dessa forma, o axioma $C$ DisjointWith $D$ se torna, após o enfraquecimento, $\left(C \sqcap \neg\left\{a_{1}\right\} \sqcap \neg\left\{a_{2}\right\} \sqcap \ldots \sqcap \neg\left\{a_{n}\right\}\right)$ DisjointWith D. O Exemplo 3.16 ilustra essa variação do mecanismo de adição de exceções.

Exemplo 3.16 Consideremos a ontologia

$$
\begin{aligned}
\mathcal{O}=\{ & \text { Mamifero DisjointWith Ave, } \\
& \text { PossuiBico } \sqsubseteq \text { Ave }, \\
& \text { Mamifero }(\text { Ornitorrinco }), \\
& \text { PossuiBico }(\text { Ornitorrinco })\} \models \top \sqsubseteq \perp
\end{aligned}
$$

O se encontra inconsistente pois o indivíduo Ornitorrinco quebra restrições a hierarquia de classes, que diz que aves e mamíferos são conceitos disjuntos e também que indivíduos que possuem bicos são aves. Porém ornitorrinco é um mamífero que possui bico. Utilizando o mecanismo de adição de exceções a axiomas de subordinação, exceções seriam adicionadas ao axioma $\alpha_{1}=$ PossuiBico $\sqsubseteq$ Ave. O resultado dessa alteração seria o axioma $\alpha_{1}^{\prime}=($ PossuiBico $\sqcap$ $\neg\{$ Ornitorrinco $\}) \sqsubseteq$ Ave, que afirma que, se existe um indivíduo que possui bico e não é o Ornitorrinco, então esse indivíduo necessariamente será uma Ave. Porém, em estudos genéticos recentes, cientistas descobriram que o Ornitorrinco, na verdade é um animal único que possui código genético de mamíferos, aves e répteis. O axioma $\alpha_{1}^{\prime}$ está errado do ponto de vista da modelagem da ontologia que tem como objetivo classificar animais em suas devidas classes biológicas de acordo com suas características. Nesse caso faz sentido que queiramos adicionar exceções ao axioma de disjunção Mamifero DisjointWith Ave, construindo o axioma (Mamifero $\square \neg\{$ Ornitorrinco\}) DisjointWith Ave, que diz que o indivíduo Ornitorrinco pode ser mamífero e Ave ao mesmo tempo.

Em [BLW06], Bonatti et al. propuseram uma estratégia para minimizar o uso de exceções. Os autores fazem uso de uma técnica chamada circunscrição de lógicas de descrição, que é uma técnica que permite a modelagem de anormalidades, ou seja, exceções. Os autores propõem uma extensão para a lógica $\mathcal{A L C} \mathcal{Q I O}$, acrescentando os chamados caminhos de circunscrição, que são predicados que capturam o comportamento anormal de certos axiomas (as exceções).

Em [CMMV13], Casini et al. apresentam resultados da utilização de lógica defeasible de descrição pode ser uma solução para lidar com problemas de conflitos, uma vez que esse tipo de lógica captura a "tipicalidade" de uma determinada consequência lógica, e.g., tipicamente pássaros voam, porém existem indivíduos que são pássaros e não voam (pinguins por exemplo). Eles utilizam em seu trabalho uma extensão para a lógica de descrição, a subsunção defeasible: $\sqsubset \approx$. Assim a base de conhecimento descrita é representada da seguinte forma: 


$$
\begin{aligned}
\mathcal{O}=\{ & \text { Passaro } \sqsubset \approx \text { Voa } \\
& \{\text { Chripy }\} \sqsubseteq \text { Passaro } \\
& \text { Pinguim } \sqsubseteq \text { Passaro } \\
& \{\text { Tweety }\} \sqsubseteq \text { Pinguim } \\
& \text { Pinguim } \nsubseteq \text { Voa }\}
\end{aligned}
$$

Embora o trabalho seja sólido e apresente alguns dados experimentais, infelizmente não faz parte dos principais mecanismos de manipulação e raciocínio em ontologias disponíveis.

\subsubsection{Enfraquecimento de Restrições de Cardinalidade}

Em adição ao mecanismo de exceções citado anteriormente, definimos uma outra atividade com a capacidade de enfraquecer restrições de cardinalidades do tipo $\leq n P$, onde $P$ é uma propriedade. Desenvolvemos um algoritmo trivial que, de forma incremental, altera o valor de $n$, realizando incrementos, permitindo que conceitos subordinados a restrição de cardinalidade se relacionem com mais que $n$ indivíduos através da propriedade $P$. O Exemplo 3.17 demonstra o funcionamento do algoritmo que propomos.

Exemplo 3.17 Consideremos a ontologia:

$$
\begin{aligned}
\mathcal{O}=\{ & C \sqsubseteq \leq 1 P, \\
& C\left(a_{1}\right), \\
& C\left(a_{2}\right), \\
& C\left(a_{3}\right), \\
& a_{1} \neq a_{3}, \\
& a_{1} \neq a_{2}, \\
& a_{3} \neq a_{2}, \\
& P\left(a_{1}, a_{2}\right), \\
& \left.P\left(a_{1}, a_{3}\right)\right\}
\end{aligned}
$$

$\mathcal{O}$ se encontra inconsistente pois o conceito $C$ está restrito a indivíduos que se relacionem com no máximo 1 outro indivíduo através da propriedade $P$, porém o indivíduo $a_{1}$ se relaciona com os indivíduos $a_{2}$ e $a_{3}$. Para resolver esse problema, podemos aumentar iterativamente o valor da restrição de cardinalidade. No caso desse exemplo, um incremento em 1, i.e., $C \sqsubseteq \leq 2$ P resolveria o problema, restaurando a consistência.

A assinatura do tipo de conflito que pode ser resolvido com essa estratégia é vista na Definição 3.2

Definição 3.2 Um conflito do tipo $\leq n P$ é definido pelos axiomas $T B o x C \sqsubseteq \leq n P$ e pelos axiomas $A B o x C\left(a_{0}\right), a_{i} \neq a_{j}, P\left(a_{0}, a_{i}\right)$, com $i$ de 1 a $n+1$. 
Uma variação deste tipo de conflito ocorre quando temos o axioma $\leq n P$ definindo um indivíduo, nesse caso o conflito do tipo $\leq n P$ é definido como o conjunto de indivíduos $\left\{a_{1}, a_{2}, \ldots\right.$, $\left.a_{n}, \ldots, a_{n+k}\right\}$ e os axiomas $A B o x(\leq n P)\left(a_{1}\right), a_{i} \neq a_{j}, P\left(a_{1}, a_{i}\right)$, onde $k>0, i \neq j$, com $j e$ $i \in 1 . . n+k$.

O número de elementos do conjunto kernel para conflitos do tipo $\leq n P$ é dado pelo número de combinações de tamanho $n+1$ dos $a_{n}$ indivíduos que se relacionam com indivíduos da classe $\mathrm{C}$ subordinada pelo axioma $\leq n P$, ou o indivíduo definido utilizando um axioma $\leq n P$. O Exemplo 3.18 é uma extensão do Exemplo 3.17 e mostra o que acontece se nós criarmos uma ontologia a partir do conjunto conflito $\mathcal{K}$ apenas adicionando um novo indivíduo $a_{4}$ que é diferente de todos os indivíduos que se relacionam com $a_{1}$ por meio da propriedade $P$.

Exemplo 3.18 Seja a ontologia

$$
\begin{aligned}
\mathcal{O}=\{ & C \sqsubseteq \leq 1 P, \\
& C\left(a_{1}\right), \\
& a_{3} \neq a_{2}, a_{4} \neq a_{2}, a_{4} \neq a_{3}, \\
& \left.P\left(a_{1}, a_{2}\right), P\left(a_{1}, a_{3}\right) P\left(a_{1}, a_{4}\right)\right\} \models \top \sqsubseteq \perp
\end{aligned}
$$

O número de conjuntos de conflitos para esta ontologia é o número de combinações de tamanho 2 dos 3 indivíduos que se relacionam com $a_{1}$ por meio da propriedade $P$, ou seja, 3 conjuntos: $\mathcal{K}_{1}=\left\{C \sqsubseteq \leq 1 P, C\left(a_{1}\right), a_{3} \neq a_{2}, P\left(a_{1}, a_{2}\right), P\left(a_{1}, a_{3}\right)\right\}, \mathcal{K}_{2}=\left\{C \sqsubseteq \leq 1 P, C\left(a_{1}\right), a_{4} \neq a_{2}\right.$, $\left.P\left(a_{1}, a_{2}\right), P\left(a_{1}, a_{4}\right)\right\}$ e $\mathcal{K}_{3}=\left\{C \sqsubseteq \leq 1 P, C\left(a_{1}\right), a_{4} \neq a_{3}, P\left(a_{1}, a_{3}\right), P\left(a_{1}, a_{4}\right)\right\}$.

No contexto de depuração de granularidade fina, infelizmente os conjuntos construídos conteriam os axiomas do conflito, em número mínimo, ou seja, $n+1$ axiomas que envolvem os $a_{i}$, com $1<i<n$ indivíduos. O enfraquecimento então resolveria o conflito apenas localmente, i.e., somente dentro de um único conjunto de conflitos. Então, após a resolução do conflito e união do conjunto de conflitos com o resto da ontologia o conflito pode surgir novamente e o usuário terá que passar por uma nova iteração do processo. Nesse caso, a utilização de conjuntos de conflitos minimais não se mostra efetiva no diagnóstico de conflitos desse tipo.

Por essa razão, o nosso processo possui uma fase de enfraquecimento, que deve fazer uso do conjunto conflito inteiro ou do conjunto remainder (completo ou parcial). No caso do enfraquecimento utilizando conjuntos kernel, construímos conjuntos de conflitos maiores, i.e., não minimais, contendo os axiomas do conflito do tipo $\leq n P$ apresentada na Definição 3.2, em seguida fariamos uma checagem sintática, contando o número de declarações de propriedades $P\left(a_{1}, a_{i}\right)$ onde $k \leq i \leq j$ e $a_{k} \neq a_{j}$ para $k \neq j$ no conjunto e alteramos o valor de $n$ para o valor obtido na checagem.

Um problema similar, ilustrado no Exemplo 3.19, ocorre com a abordagem de adição de exceções de Qi et al. [QLB06]. O exemplo mostra que o uso do conjunto kernel também não é eficaz para a resolução de conflitos utilizando tal técnica. 
Exemplo 3.19 Seja a ontologia:

$$
\begin{aligned}
& \mathcal{O}=\{C \sqsubseteq D, \\
& C\left(a_{1}\right), \\
& \neg D\left(a_{1}\right), \\
& C\left(a_{2}\right), \\
& \neg D\left(a_{2}\right), \\
&\left.a_{1} \neq a_{2}\right\} \models \top \sqsubseteq \perp
\end{aligned}
$$

O conjunto kernel calculado, supondo $\alpha=\top \sqsubseteq \perp e ́$ :

$$
\begin{aligned}
\mathcal{O} \Perp(\top \sqsubseteq \perp)=\{ & \left\{C\left(a_{1}\right), \neg D\left(a_{1}\right), C \sqsubseteq D\right\}, \\
& \left.\left\{C\left(a_{2}\right), \neg D\left(a_{2}\right), C \sqsubseteq D\right\}\right\}
\end{aligned}
$$

Caso o usuário escolha enfraquecer o conjunto, em $\mathcal{K}_{1}$ obtemos $\mathcal{K}_{1}^{\prime}=\left\{C\left(a_{1}\right), \neg D\left(a_{1}\right),(C \sqcap\right.$ $\left.\left.\neg\left\{a_{1}\right\}\right) \sqsubseteq D\right\} ;$ em $\mathcal{K}_{2}$ obtemos $\mathcal{K}_{2}^{\prime}=\left\{C\left(a_{2}\right), \neg D\left(a_{2}\right),\left(C \sqcap \neg\left\{a_{2}\right\}\right) \sqsubseteq D\right\}$. Ao unirmos as duas soluções verificamos um problema, pois $a_{1} \neq a_{2}, C \sqcap \neg\left\{a_{1}\right\} \sqsubseteq D$ e $C\left(a_{2}\right) \models D\left(a_{2}\right)$. Os axiomas $C \sqcap \neg\left\{a_{1}\right\} \sqsubseteq D$ e $C \sqcap \neg\left\{a_{2}\right\} \sqsubseteq D$ devem ser unidos em $C \sqcap \neg\left\{a_{1}\right\} \sqcap \neg\left\{a_{2}\right\} \sqsubseteq D$.

No exemplo anterior a solução local resultou em inconsistência na hora de atualizar $\mathcal{O}$, pois se $a_{1} \neq a_{2}, C \sqcap \neg\left\{a_{1}\right\} \sqsubseteq D$ e $C\left(a_{2}\right)$ estamos dizendo que o indivíduo $a_{2}$, que é diferente de $a_{1}$, tem que ser necessariamente do tipo $D$, porém o axioma $C \sqcap \neg\left\{a_{1}\right\} \sqsubseteq D$ diz que somente $a_{1}$ pode pertencer ao tipo $C$ sem pertencer ao $D$. Daí a necessidade da fusão dos axiomas que adicionam exceções.

No caso da alteração no valor de $n$ nos conflitos $\leq n P$, a atualização resulta em bases ainda contendo a inferência indesejada. Para resolver isso, precisamos realizar a fusão dos conjuntos enfraquecidos, como visto no Exemplo 3.19, obtendo uma representação mais concisa das exceções, e, no caso da atualização no valor da cardinalidade, precisamos incrementar em 1 o valor de $n$ para cada elemento do conjunto kernel que também teve seu conflito resolvido com o enfraquecimento, assim como foi mostrado no exemplo 3.18, se resolvêssemos apenas um dos $\alpha$-kernel, alterando o valor da restrição $\leq n P$, não resolveríamos todos os conflitos.

No caso da utilização de conjuntos resíduo, o uso de ambas formas de enfraquecimento se dá através da análise do elemento do conjunto resíduo selecionado e os elementos excluídos, i.e., $\mathcal{O} \backslash \mathcal{R}_{i}$. Caso a junção de algum deles com o conjunto resíduo resulte em um conflito do tipo $\leq n P$, o mesmo pode ser enfraquecido com a técnica descrita. De forma análoga, caso o conflito possa ser resolvido com a adição de exceções, o mesmo será feito.

Conflitos envolvendo axiomas do tipo $\geq n P$ ocorrem quando existem declarações explicitamente opostas ao axioma $\geq n P$, e.g., se tivermos o seguinte conjunto de conflitos $\mathcal{K}=\{C \sqsubseteq \geq 3 P$, $\left.C\left(a_{1}\right),(\leq 2 P)\left(a_{1}\right)\right\}$. Nesse caso, nós temos declarações conflitantes, provavelmente advindas de uma modelagem mal feita da ontologia. Esse tipo de conflito não pode ser resolvido utilizando a mesma abordagem que foi utilizada para a solução de conflitos do tipo $\leq n P$. Para solucionar esse tipo de conflito, nós propomos uma estratégia simples: alterar o valor de $n$ em $\geq n P$ para o mesmo de $\leq n P$, i.e., atribuindo a $n$ o maior dos valores, restaurando então a consistência. Caso fosse feito de forma contrária, poderíamos cair em um conflito do tipo $\leq n P$ e que após resolvido resultaria 
em um conflito $\geq n P$, e.g., imagine a ontologia $\mathcal{O}=\left\{(\leq 2 P)\left(a_{1}\right), C\left(a_{1}\right), C \sqsubseteq \geq 3 P, P\left(a_{1}, a_{2}\right)\right.$, $\left.P\left(a_{1}, a_{3}\right), P\left(a_{1}, a_{4}\right), a_{1} \neq a_{2} \neq a_{3} \neq a_{4}\right\}$, ao resolvermos o conflito, atribuindo a $n$ o menor dos valores, o de $a$ obteríamos a ontologia $\mathcal{O}^{\prime}=\left\{(\leq 2 P)\left(a_{1}\right), C\left(a_{1}\right), C \sqsubseteq \geq 2 P, P\left(a_{1}, a_{2}\right), P\left(a_{1}, a_{3}\right)\right.$, $\left.P\left(a_{1}, a_{4}\right), a_{1} \neq a_{2} \neq a_{3} \neq a_{4}\right\}$, que possui um conflito do tipo $\leq n P$

A execução dessa fase tem como objetivo a resolução de conflitos através do enfraquecimento de restrições em fórmulas. Essa fase pode ser executada várias vezes, assim o usuário pode combinar as diversas técnicas de enfraquecimento. Porém nem sempre é possível ou desejável resolver o problema de conflitos em ontologias através do enfraquecimento de axiomas. A próxima fase do processo proposto resolve o problema de conflitos por meio da remoção mínima de axiomas.

\subsection{Remoção de Axiomas}

Em casos de depurações de granularidade fina, nem sempre é possível remover a inferência indesejada somente com o uso de técnicas de enfraquecimento. Nesses casos, utilizamos junto com a estratificação uma função de incisão que selecionará os elementos menos preferidos do estrato de último nível, removendo assim a informação menos confiável da ontologia.

\subsection{Conclusão}

O processo proposto tem como objetivo resolver problemas resultantes da fusão de ontologias. Definimos aqui a natureza desses problemas (inferências indesejadas). Em seguida separamos a tarefa de depuração em duas, uma onde o usuário possui total controle das causas do conflito (depuração de granularidade fina) e outra onde o processo sugere uma ou várias alternativas de resolução de conflito (depuração de granularidade grossa).

Após a depuração, descrevemos algumas das formas de estratificação de conjuntos conflitos mais comuns descritas na literatura. Realizamos a construção de suas versões para a aplicação em conjuntos resíduos via funções de seleção.

Descrevemos também formas de resolver conflitos sem necessariamente descartar o axioma inteiro (justificativas de granularidade fina) ou apenas adicionando informações extras ao axiomas (adição de exceções e enfraquecimento de restrições $\leq n P$ ).

Por fim, no caso da depuração de granularidade fina, caso não possamos resolver o conflito via enfraquecimento, sugerimos a remoção do(s) axioma(s) menos relevante(s) - selecionados via uma função de incisão.

No próximo capítulo mostraremos algumas construções de operadores para a obtenção de conjuntos resíduos propostas por nós. Mostraremos também dados de experimentos realizados tanto em ontologias reais quanto com ontologias construídas automaticamente. 


\section{Capítulo 4}

\section{Detalhamento das Construções do Conjunto Resíduo}

Nesse Capítulo apresentaremos em detalhe as heurísticas que propomos para a melhoria de desempenho do algoritmo para construção do conjunto resíduo. Detalhamos o projeto de nosso arcabouço, no qual implementamos as heurísticas propostas e por fim mostramos os resultados obtidos em uma série de experimentos realizados utilizando nosso arcabouço, recebendo como entrada dados gerados de forma automática e dados reais extraídos do $\mathrm{NCBO}^{1}$ BioPortal $^{2}$.

\subsection{Otimizações Durante a Fase de Encolhimento}

Nessa seção apresentaremos os algoritmos que utilizamos para otimizar a fase de encolhimento.

\subsubsection{Janelas Deslizantes}

Assim como proposto por Kalyanpur [Kal06], a construção de conjunto resíduo também pode se beneficiar da abordagem de janelas deslizantes. A ideia é a mesma aplicada do encolhimento Black-box clássico, porém a cada passo um conjunto de axiomas é removido por vez. O algoritmo pode ser visto na Listagem 2.7 .

Durante a execução do algoritmo estamos construindo um conjunto do zero que tem que ser maximal, portanto quanto mais elementos conseguirmos incluir nesse novo conjunto de uma única vez, melhor. Na abordagem Bottom-up temos um cenário invertido: realizamos a busca pelo primeiro axioma, que, caso removido, invalide a inferência indesejada. Nessa abordagem não há nenhum benefício na remoção de múltiplos axiomas de uma única vez, pois isso acarretaria em um conjunto não maximal.

O algoritmo de janelas deslizantes para a construção de elementos do conjunto resíduo pode ser visto na Listagem 4.1.

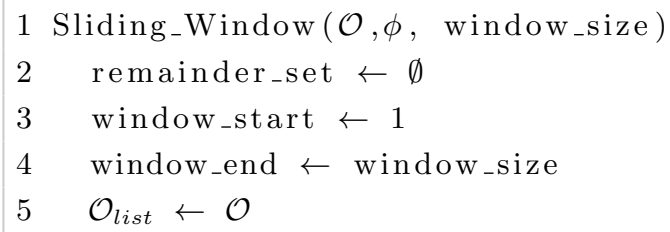

\footnotetext{
${ }^{1}$ http://www.bioontology.org/

${ }^{2}$ http://bioportal.bioontology.org/
} 


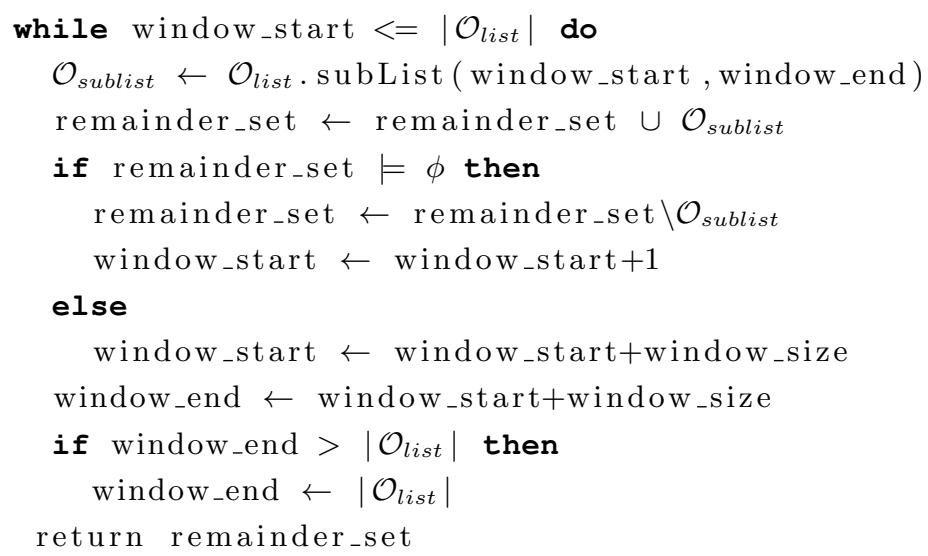

Listagem 4.1: Algoritmo Janelas Deslizantes para cálculo de elementos do conjunto Resíduo

O funcionamento desse algoritmo pode ser visto no Exemplo 4.1.

Exemplo 4.1 Considerando a ontologia do Exemplo 2.9, utilizando uma janela deslizante de tamanho 3, teríamos os seguintes passos de execução:

$$
\mathcal{O}=\{A \sqsubseteq B, B \sqsubseteq D, A \sqsubseteq E, E \sqsubseteq F\}
$$

Posicionando a janela no primeiro axioma obteríamos:

$$
\mathcal{O}=\{A \sqsubseteq B, B \sqsubseteq D, A \sqsubseteq E, E \sqsubseteq F\}
$$

O fragmento extraído utilizando a janela deslizante ainda infere $A \sqsubseteq D$, portanto a janela deve deslizar uma posição, indo para a configuração:

$$
\mathcal{O}=\{A \sqsubseteq B, B \sqsubseteq D, A \sqsubseteq E, E \sqsubseteq F\}
$$

A partir desse fragmento não podemos mais inferir que $A \sqsubseteq D$.

\subsubsection{Conectividade Estrutural}

Assim como suposto em [Was99], uma função guiada que seleciona os elementos que serão removidos - no caso do encolhimento - ou os que serão adicionados - no caso da expansão pode ser de grande utilidade, sobretudo em dados reais, uma vez que, comumente, os elementos causadores da inferência indesejada são ligados estruturalmente à inferência. $\mathrm{O}$ algoritmo para a expansão utilizando conectividade sintática pode ser visto na Listagem 4.2.

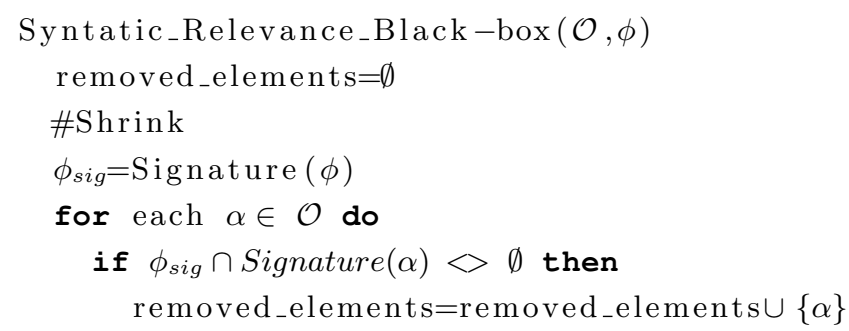




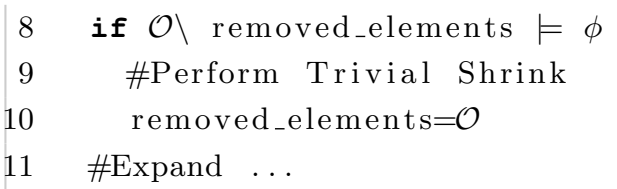

Listagem 4.2: Algoritmo baseado na conectividade sintática para fase de expansão durante o cálculo de um elemento do conjunto resíduo

O algoritmo mostrado na Listagem 4.2 depende da função Signature que devolve uma lista com todos os conceitos presentes no axioma passado como parâmetro.

\subsection{Otimizações Durante a Fase de Expansão}

Durante essa Seção mostraremos os algoritmos utilizados para implementar as heurísticas propostas nesse trabalho.

\subsubsection{Divisão e Conquista}

Inspirado na ideia de [Jun01], desenvolvemos um algoritmo baseado no paradigma divisão e conquista para a construção de elementos do conjunto resíduo. O algoritmo desenvolvido é bem similar ao Black-box clássico que utiliza divisão e conquista na fase de encolhimento. Diferença entre os dois métodos está no fato que não estamos procurando de forma mais rápida o núcleo responsável pela inferência indesejada, mas uma forma mais rápida de construir um conjunto maximal onde a inferência não seja válida. O algoritmo pode ser visto na Listagem 4.3.

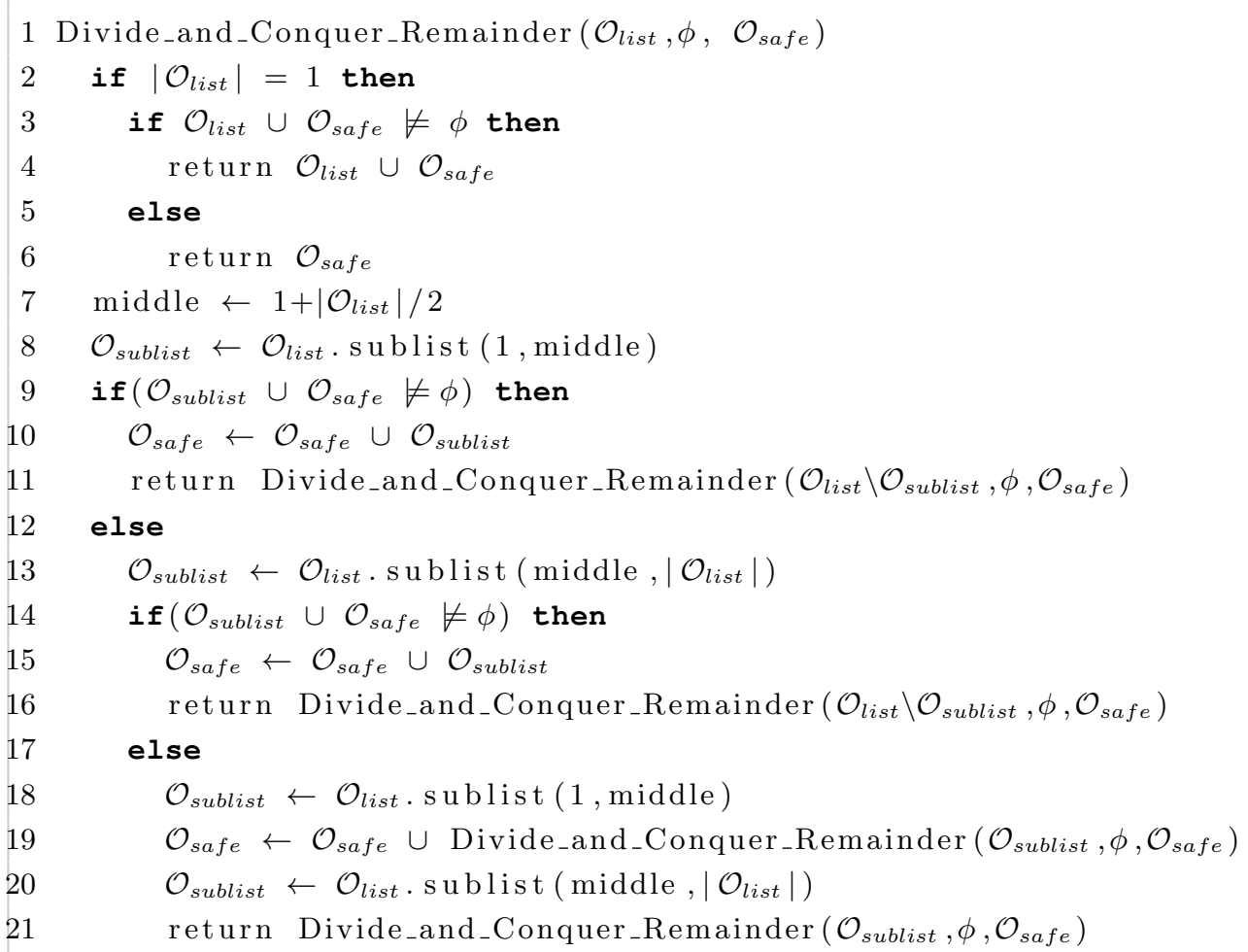

Listagem 4.3: Algoritmo baseado em Divisão e Conquista para cálculo de elementos do conjunto Resíduo

O Exemplo 4.2 ilustra o funcionamento do algoritmo. 
Exemplo 4.2 Vamos considerar a seguinte ontologia:

$$
\begin{aligned}
\mathcal{O}=\{A & \sqsubseteq B \\
B & \sqsubseteq D \\
A & \sqsubseteq F \\
F & \sqsubseteq D\}
\end{aligned}
$$

Suponhamos então que a inferência indesejada seja $\phi=A \sqsubseteq D$. O algoritmo teria início dividindo $\mathcal{O}$ em $\mathcal{O}_{1}=\{A \sqsubseteq B, B \sqsubseteq D\}$ e verificaria se a partir dessa sub-ontologia seria possivel inferir $\phi$ (linha 9). Esse é o caso, então o algoritmo tentaria utilizar a segunda metade da base, i.e., $\mathcal{O}_{2}=\{A \sqsubseteq F, F \sqsubseteq D\}$, que ainda consegue inferir $\phi$. O algoritmo então executaria o conjunto de comandos da cláusula else da linha 17.

Dessa forma o programa faria uma chamada recursiva, dessa vez utilizando não a ontologia inteira, mas $\mathcal{O}_{1}$, que seria dividido em $\mathcal{O}_{11}=\{A \sqsubseteq B\}$, que por sua vez não infere $\phi . \mathcal{O}_{11}$ é armazenado então em $\mathcal{O}_{\text {safe }}$ e o algoritmo fará uma nova chamada recursiva, agora com $\mathcal{O}_{12}=$ $\{B \sqsubseteq D\}$. Nessa chamada temos o caso especial em que $\mathcal{O}_{\text {list }}$ é unitário (linha 2), então verificamos se esse axioma pode ser incluído na resposta de forma segura (linha 3). Como esse não é o caso, essa pilha de chamadas recursivas retorna com o valor $\{A \sqsubseteq B\}$.

Devemos agora processar a segunda metade da ontologia, i.e., $\mathcal{O}_{2}$ e uma nova chamada recursiva a esse algoritmo é realizada. Dessa vez com a exigência que o resultado seja compatível que o que foi acumulado em $\mathcal{O}_{\text {safe }}$ (linha 21). Durante a chamada recursiva $\mathcal{O}_{2}$ é dividida em $\mathcal{O}_{21}=\{A \sqsubseteq F\}$, que junto de $\mathcal{O}_{\text {safe }}$ não infere $\phi$ e portanto pode ser incluída de forma segura em $\mathcal{O}_{\text {safe }}$ (linha 15$)$.

Por fim, a última chamada recursiva é executada com as entradas $\mathcal{O}_{\text {safe }}=\{A \sqsubseteq B, A \sqsubseteq F\}$, $\mathcal{O}_{\text {list }}=\{F \sqsubseteq D\}$, onde é verificado que $\mathcal{O}_{\text {list }}$ não pode ser adicionado a $\mathcal{O}_{\text {safe }}$ diretamente, o que não é o caso (linha 3). Dessa forma o método retorna o conteúdo de $\mathcal{O}_{\text {safe, }}$ um conjunto maximal que não infere $\phi$.

Esse foi o pior caso em termos de ordenação de axiomas e precisamos de um número superior em relação à abordagem proposta por Resina et al. [RRW14] de chamadas ao mecanismo de inferências para poder construir um elemento do conjunto resíduo. Entretanto, em uma outra forma de ordenação, como por exemplo $\mathcal{O}=\{A \sqsubseteq B, A \sqsubseteq F, B \sqsubseteq D, F \sqsubseteq D\}$ teríamos um número diferente de chamadas ao mecanismo de inferências. Nossos experimentos realizados com dados gerados e dados reais apontam que ocorre uma redução substancial no número de chamadas ao mecanismo de inferência, como pode ser visto na Seção 4.4 .

\subsection{Arcabouço para Definição de Operadores para Cálculo de Conjuntos Resíduo e Kernel}

Com o objetivo de testar os operadores propostos no Capítulo 4, desenvolvemos um arcabouço com entidades responsáveis pela construção de conjuntos resíduo e kernel. O arcabouço foi desenvolvido em Java e utiliza OWLAPI para a manipulação de ontologias. O Diagrama UML de classes, mostrado na Figura 4.1 descreve a arquitetura geral da ferramenta.

O principal componente nessa arquitetura é o BlackBox, esse componente encapsula o funci- 


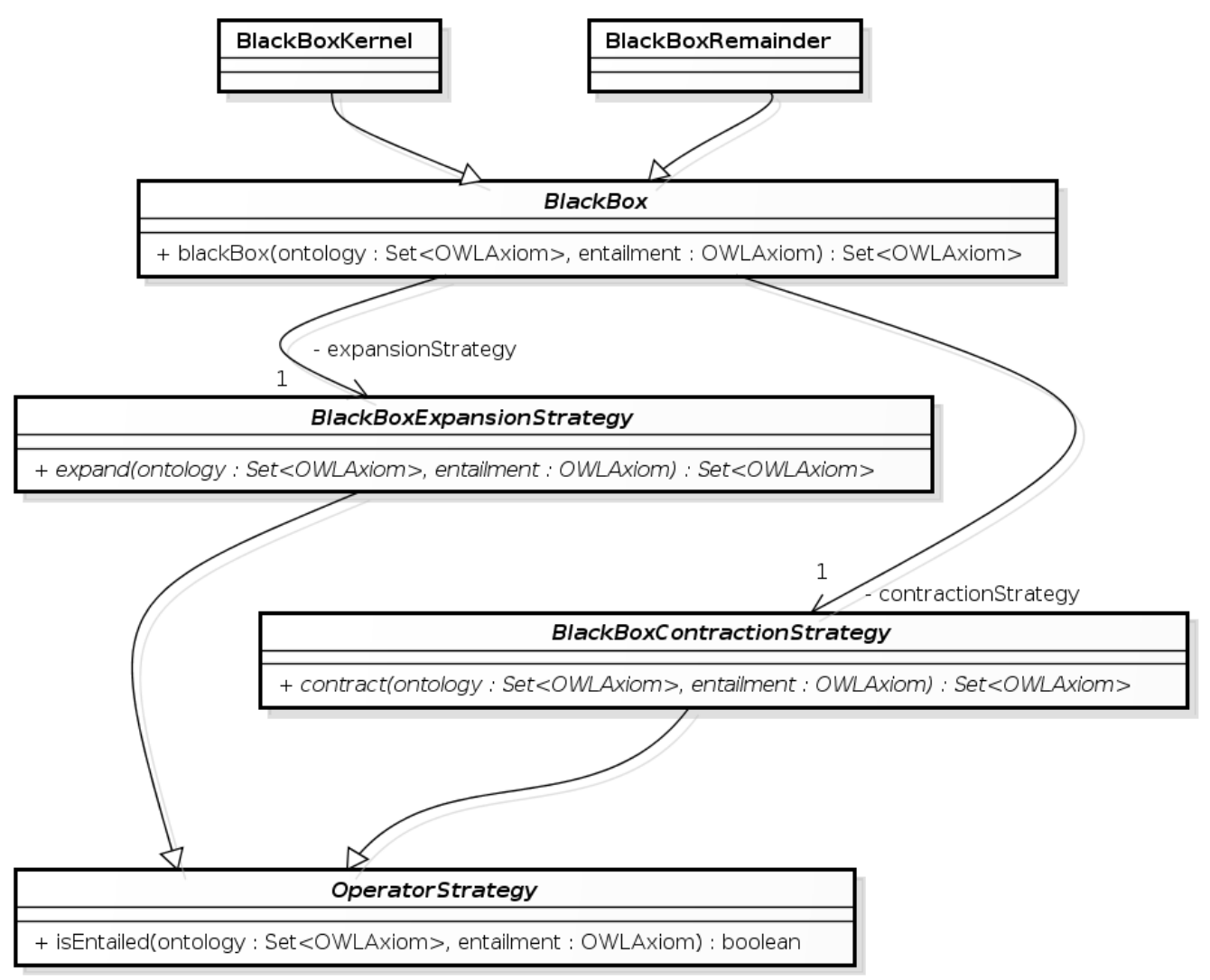

Figura 4.1: Arquitetura Geral do Framework

onamento de um algoritmo Black-box. Essa classe possui duas especializações, uma que utiliza a estratégia expande-encolhe (BlackBoxKernel) e encolhe-expande(BlackBoxRemainder). Essa classe é abstrata e somente as duas especializações devem ser utilizadas. Uma única operação deve ser implementada para criar um novo operador Black-box, a operação blackBox que recebe um conjunto de axiomas e um axioma que representa a inferência indesejada.

Tipicamente o algoritmo Black-box utiliza dois outros componentes, uma estratégia para o encolhimento (BlackBoxContractionStrategy) e uma estratégia para a expansão (BlackBoxExpansionStrategy). Ambos componentes possuem uma única operação que deve ser implementada para a definição de novas estratégias de expansão ou encolhimentos. São elas: contract, para a contração que recebe um conjunto de axiomas e um axioma (inferência indesejada) e produz um subconjunto do conjunto recebido como parâmetro; e expand, para a expansão que também recebe um conjunto de axiomas e um axioma e produz também um subconjunto do conjunto recebido como parâmetro.

Do ponto de vista da construção de conjuntos kernel, a Figura 4.2 mostra os componentes desenvolvidos para cálculo de um único $\alpha$-kernel.

Na Figura 4.2, é possível ver o conjunto de operadores responsáveis pela fase de expansão:

- ClassicalBlackBoxKernelExpansionStrategy: estratégia básica de expansão, onde os elementos são adicionados um a um, até a obtenção da inconsistência;

- SyntaticRelevanceBlackboxKernelExpansionStrategy: estratégia na qual os elementos conectados sintaticamente aos elementos já no $\alpha$-kernel são adicionados.

Também é possível ver os operadores responsáveis pela fase de encolhimento: 


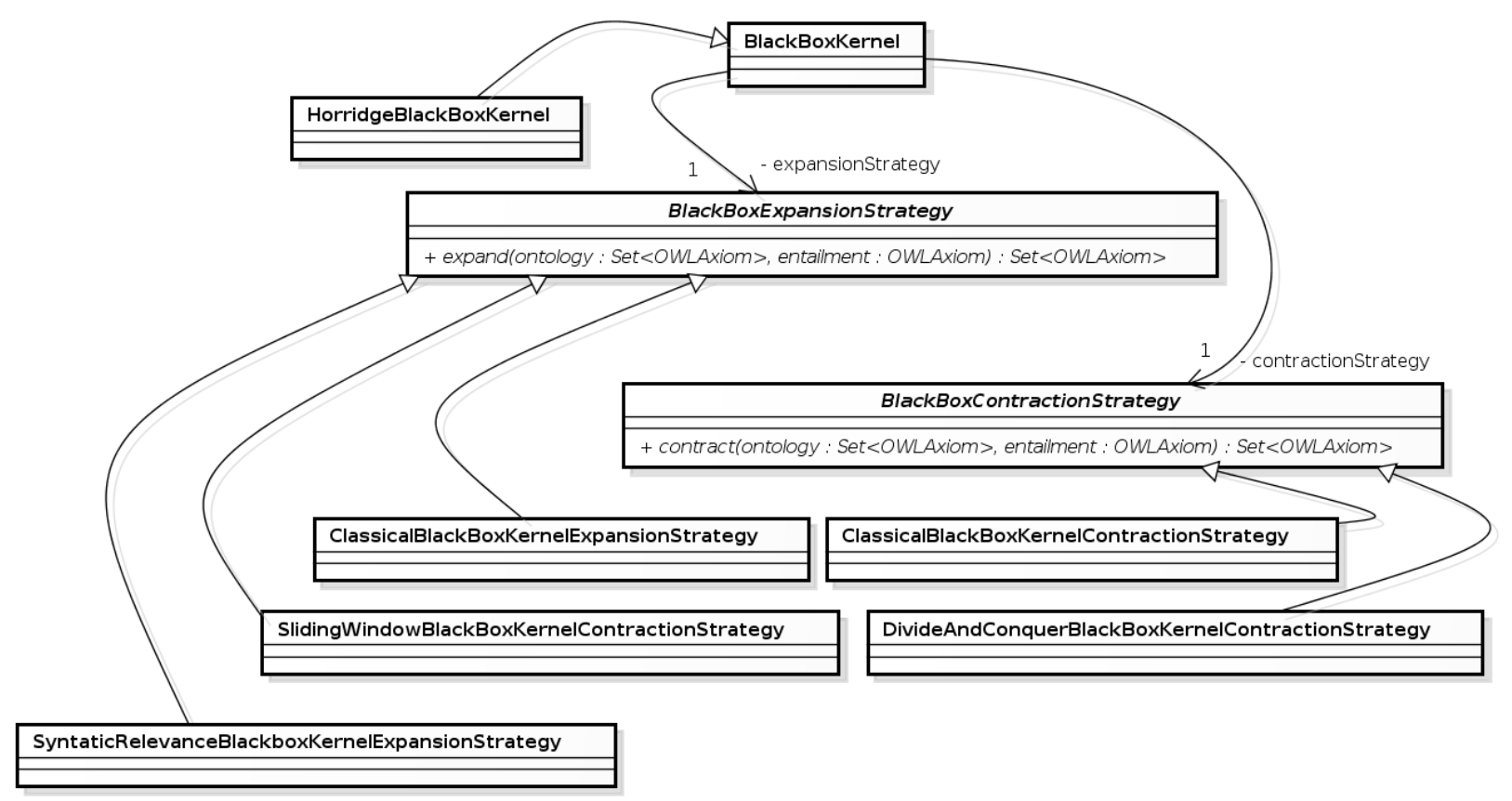

Figura 4.2: Arquitetura de Componentes para a construção de Elementos do Conjunto Kernel

- SlidingWindowBlackBoxKernelContractionStrategy: estratégia na qual mais do que um elemento são removidos por vez durante o encolhimento;

- DivideAndConquerBlackBoxKernelContractionStrategy: estratégia na qual a ontologia é subdividida recursivamente até encontrar o $\alpha$-kernel;

- ClassicalBlackBoxKernelContractionStrategy: técnica de contração clássica onde os elementos são removidos um a um da sub-ontologia conflitante;

No diagrama da Figura 4.2, também podemos ver o componente HorridgeBlackBoxKernel. Esse componente é responsável por construir um $\alpha$-kernel utilizando a implementação disponível no OWLExplanation plugin.

As implementações para os componentes que fazem a construção de $\alpha$-kernels estão disponíveis na literatura. Utilizamos como referência, a descrição das otimizações listadas em [Hor11].

Além dos operadores para a construção dos $\alpha$-kernels, implementamos também, em nosso arcabouço, operadores para a construção de um único item do conjunto resíduo. O relacionamento entre esses componentes pode ser visto no diagrama UML visto na Figura 4.3.

É importante notar a diferença entre as duas arquiteturas. No caso do conjunto resíduo, precisamos especializar as classes BlackBoxExpansionStrategy e BlackBoxContractionStrategy. Isso se faz necessário pois para realizar a expansão após o encolhimento, precisamos manter uma lista dos axiomas excluídos durante o encolhimento. Portanto, introduzimos a capacidade de guardar os dados removidos na primeira fase.

Na Figura 4.3, é possível ver o conjunto de operadores responsáveis pela fase de expansão:

- ClassicalBlackBoxRemainderExpansionStrategy: estratégia básica de expansão, onde os elementos recém removidos são readicionados um a um; 


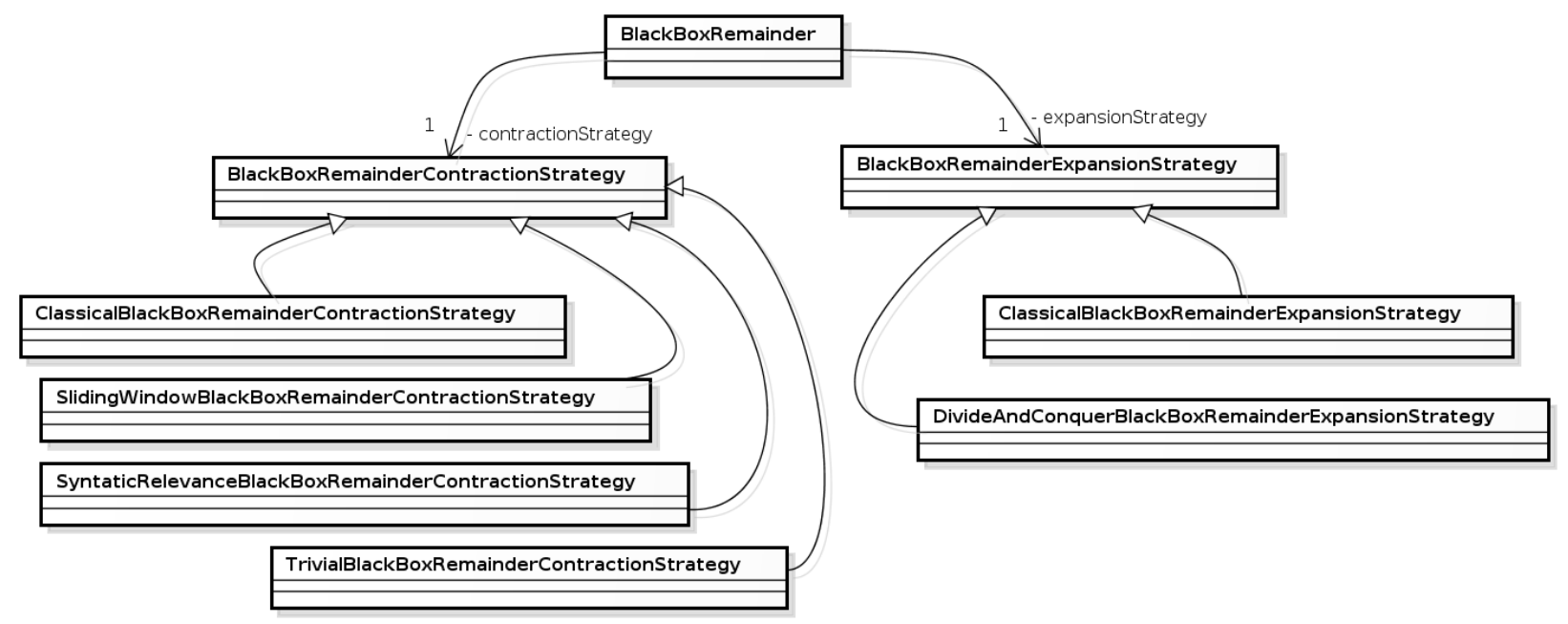

Figura 4.3: Arquitetura de Componentes para a construção de Elementos do Conjunto Resíduo

- DivideAndConquerBlackBoxRemainderExpansionStrategy: estratégia na qual os elementos recém removidos são divididos recursivamente e readicionados até que o maior subconjunto do que foi removido possa ser adicionado sem que seja possível realizar a inferência indesejada;

Também é possível ver os operadores responsáveis pela fase de encolhimento:

- ClassicalBlackBoxRemainderContractionStrategy: os elementos são removidos um a um da ontologia, até que a inferência indesejada não seja mais válida;

- TrivialBlackBoxRemainderContractionStrategy: estratégia trivial, na qual a ontologia inteira é removida durante o encolhimento;

- SlidingWindowBlackBoxRemainderContractionStrategy: técnica de contração que remove um conjunto de elementos por vez, até que a inferência indesejada não seja mais válida;

- SyntaticRelevanceBlackBoxRemainderContractionStrategy: estratégia que remove primeiro os axiomas que estão conectados sintaticamente aos conceitos contidos na inferência indesejada.

Com essa separação de componentes fica fácil a mescla de estratégias para obtenção do melhor resultado. A Listagem 4.4 mostra como é possível mesclar estratégias de expansão/enconlhimento, utilizando nosso arcabouço tanto na construção de operadores Black-box kernel quanto conjunto resíduo.

1 OWLontologyManager manager = OWLManager.createowLontologyManager();

2 OWLontology ontology = manager.loadontologyFromontologyDocument (new File(" ontology. owl"));

3

4 / /First the Remainder

5 BlackBox remainderElementBuilder = new BlackBoxRemainder (new

DivideAndConquerBlackBoxRemainderExpansionstrategy (manager),

6 new SyntaticRelevanceBlackBoxRemainderContractionstrategy (manager));

7 Set<OWLAxiom> remainderElement = remainderElementBuilder.blackBox(ontology. getAxioms (), entailment);

8

9 / Now the $\alpha$-kernel 


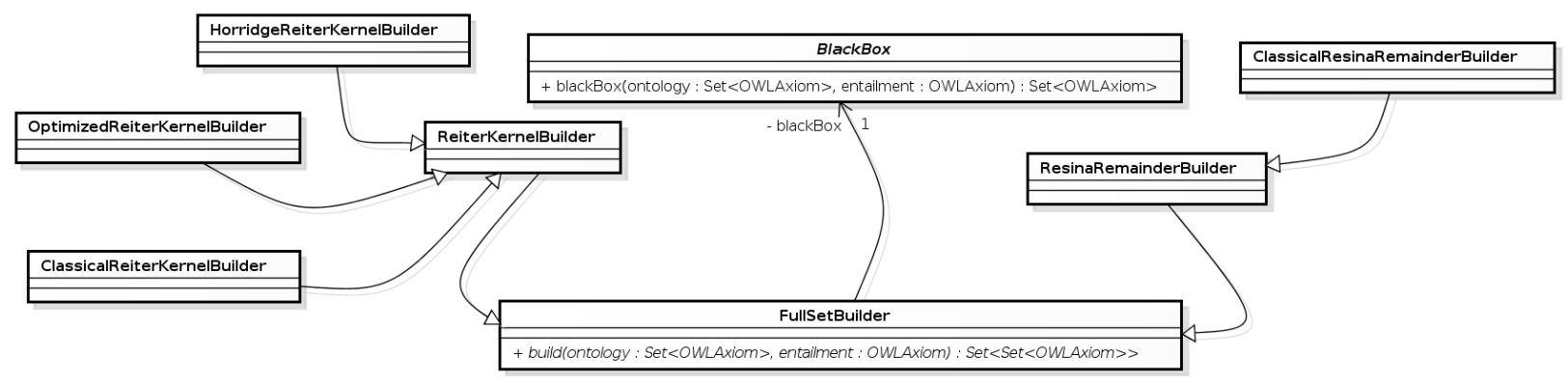

Figura 4.4: Arquitetura do arcabouço com componentes para a construção de kernel e resíduo completos

10

11

12

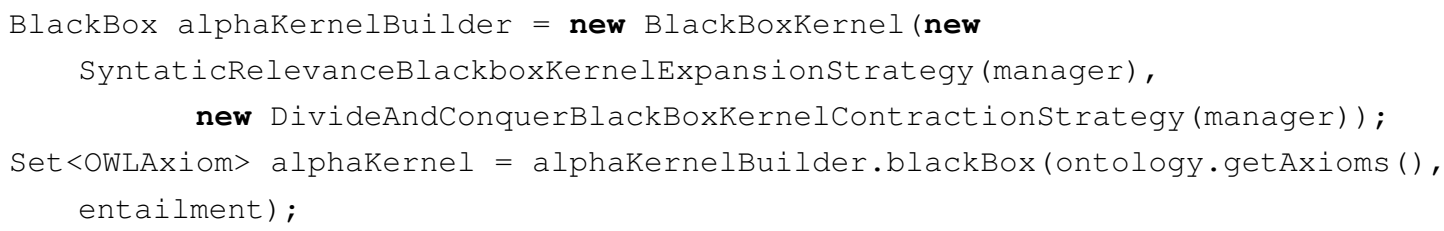

Listagem 4.4: Código exemplo que mostra a criação de novos operadores Black-box para cálculo de $\alpha$-kernel e itens no conjunto resíduo

\subsubsection{Construção do Conjunto Kernel e Resíduo}

No nosso arcabouço incluímos componentes que constroem os conjuntos kernel e resíduo completos. Esses componentes não foram utilizados nos testes realizados pois, para o caso da construção do conjunto kernel, o desempenho obtido não se comparava ao desempenho da ferramenta OWLExplanation plugin. Observamos os ganhos das otimizações descritas por Horridge, em [Hor11], porém, nossa implementação não conseguiu competir com o OWLExplanation plugin, que faz uso intensamente de funções da OWLAPI e já está sendo desenvolvido por anos (desde 2011).

No caso do algoritmo para a construção do conjunto resíduo inteiro, nossos primeiros experimentos mostraram que a simples construção de um elemento do conjunto demorava um tempo compatível com a construção do conjunto kernel completo, utilizando o OWLExplanation plugin. Por essa razão, descartamos os testes com a geração completa do conjunto resíduo.

A arquitetura do arcabouço para a construção de conjuntos completos pode ser vista na Figura 4.4 .

Na Figura 4.4, a principal interface para a construção de conjuntos kernel e resíduo completos é o FullSetBuilder. Esse componente possui apenas uma única operação, que deve ser implementada para a criação de um novo componente capaz de gerar conjuntos completos, o método build, que recebe um conjunto de axiomas como parâmetro e gera um conjunto de conjuntos ( $\alpha$-kernels ou itens do conjunto resíduo).

$\mathrm{Na}$ arquitetura proposta temos duas formas básicas de conjuntos que desejamos construir, o conjunto kernel e o conjunto resíduo. Mais especificamente, temos duas formas de construir esses conjuntos, o kernel via algoritmo de Reiter [Rei87] e o resíduo via algoritmo proposto por Resina et. al. [RRW14].

No caso da geração de conjuntos kernel baseada no algoritmo de Reiter [Rei87], criamos alguns operadores:

- ClassicalReiterKernelBuilder: contém o algoritmo como definido em [Was00]; 
- OptmizedReiterKernelBuilder: contém o algoritmo clássico com as otimizações de fechamento precoce de caminhos e reúso de nós descritas em [Hor11]; e

- HorridgeReiterKernelBuilder: funciona como um adaptador para a implementação OWLExplanation plugin para cálculo de conjuntos kernel completos;

No caso da construção do conjunto resíduo, apenas disponibilizamos o algoritmo descrito em [RRW14]. Deixamos espaço aqui para otimizações futuras ao proposto por Resina et al. [RRW14].

O arcabouço desenvolvido se encontra disponível, como software livre em https://github.com/ raphaelmcobe/ontology-debug-and-repair.

Os algoritmos utilizados para a implementação dos componentes para a construção de conjuntos kernel se encontram detalhados no Capítulo 2. No próximo capítulo mostraremos os algoritmos propostos nesta tese para a construção de conjuntos resíduo. Mostraremos também dados obtidos do experimento conduzido com o objetivo de avaliar o desempenho dos algoritmos lá descritos.

\subsection{Experimentos}

Com o objetivo de investigar o comportamento dos operadores que propusemos no Capítulo 3 realizamos dois tipos de experimentos, o primeiro utilizando dados gerados de forma automática e o segundo utilizando dados reais. Ambos experimentos foram executados 5 vezes e os valores apresentados representam a média das 5 execuções.

Para a realização desses experimentos estabelecemos um conjunto de hipóteses, cuja comprovação tem como objetivo mostrar que as heurísticas propostas causam impacto positivo na construção do conjunto resíduo.

\subsubsection{Hipóteses}

O conjunto de hipóteses levantado é composto de:

H1: A utilização das heurísticas propostas melhoram o desempenho em algum aspecto: essa questão é respondida analisando as métricas de número de chamadas ao mecanismo de inferência, memória consumida pela execução e o tempo total utilizado pelo algoritmo.

H2: Existem casos onde construir um elemento do conjunto resíduo é tão ou menos custoso do que construir um elemento de conjunto kernel: queremos detectar casos extremos onde a construção de um único elemento do conjunto kernel deve utilizar mais recursos - memória, chamadas ao mecanismo de inferências ou tempo de execução - do que a construção de um único elemento do conjunto resíduo. Argumentamos que a existência desses casos justifica a adoção de uma estratégia de depuração com granularidade grossa, onde o sistema sugere uma solução para a inferência indesejada;

H3: Existem casos onde construir o conjunto kernel é tão ou mais custoso do que construir um elemento do conjunto resíduo: mesmo que em muitos casos, construir um elemento do conjunto kernel seja mais fácil do que construir um elemento do conjunto resíduo, após a construção temos apenas um foco de conflito. Ainda é necessário, para a depuração a construção de todos os elementos do conjunto kernel. Assim, com essa hipótese buscamos confirmar que existem 
casos onde se é mais fácil construir um elemento do conjunto resíduo do que o conjunto kernel inteiro.

\subsubsection{Métricas Coletadas}

As métricas coletadas utilizadas para validação de nossas hipóteses foram:

1. Número de chamadas ao reasoner: o número de chamadas ao mecanismo de inferências é uma métrica chave na comparação de operadores, uma vez que algoritmos para a classificação e/ou checagem de consistência em lógicas de descrição possuem alta complexidade. Assim, um bom desempenho de operador Black-box pode estar restrito ao número de chamadas ao mecanismo de inferências que o mesmo faz.

2. Tempo total da execução: durante nossos experimentos verificamos que nem sempre o número de chamadas ao mecanismo de inferências é o fator determinante para estabelecer que um algoritmo possui melhor desempenho que outro. Em alguns casos, a ordem de complexidade do próprio algoritmo é o gargalo para a construção do conjunto kernel ou resíduo.

3. Memória utilizada pela Máquina Virtual Java durante a execução do teste: monitoramos com essa métrica a quantidade de memória consumida pelo algoritmo Black-box incluso o uso por parte do mecanismo de inferências bem como o consumo por parte do algoritmo.

\subsubsection{Design do Experimento}

Para o caso do cálculo de um único elemento do conjunto kernel, utilizamos a seguinte combinação de operadores Black-box:

1. Expansão Clássica e Encolhimento Clássico;

2. Expansão Clássica e Encolhimento via Janelas Deslizantes;

3. Expansão Clássica e Encolhimento via Divisão e Conquista;

4. Expansão utilizando conectividade Sintática e Encolhimento Clássico;

5. Expansão utilizando conectividade Sintática e Encolhimento utilizando Janelas Deslizantes;

6. Expansão utilizando conectividade Sintática e Encolhimento utilizando Divisão e Conquista.

Para o cálculo de um único elemento do conjunto resíduo, utilizamos a seguinte combinação de operadores Black-box:

1. Expansão Clássica e Encolhimento Clássico;

2. Expansão Clássica e Encolhimento via Janelas Deslizantes;

3. Expansão Clássica e Encolhimento via Conectividade Sintática;

4. Expansão Clássica e Encolhimento Trivial (remoção de todos os axiomas da ontologia);

5. Expansão via Divisão e Conquista e Encolhimento Trivial; 
6. Expansão via Divisão e Conquista e Encolhimento via Conectividade Sintática;

Tentamos realizar todas as combinações de estratégias de otimização possíveis, entretanto, testes iniciais mostraram que a combinação da otimização Expansão via Divisão e Conquista com Encolhimento Clássico e via Janelas Deslizantes ultrapassava o período de timeout definido para os experimentos, tanto no caso dos dados gerados como no caso dos dados do BioPortal.

Para o cálculo do conjunto kernel inteiro utilizamos a implementação utilizada no OWLExplanation plugin ${ }^{3}$. Essa implementação foi o resultado do trabalho [Hor11] e representa o atual estado da arte no que diz respeito a depuração de ontologias. O autor afirma que nessa implementação utilizou as heurísticas de otimização: Black-box com expansão utilizando conectividade sintática, encolhimento utilizando divisão e conquista; Reiter utilizando reuso de nós e fechamento precoce de caminhos;

\subsubsection{Validação dos Resultados}

Para validar os resultados retornados pelos operadores criamos as seguintes rotinas: validação de um único elemento do kernel - Listagem 4.5; validação de um único elemento do conjunto resíduo - Listagem 4.6; e validação do conjunto kernel inteiro - 4.7

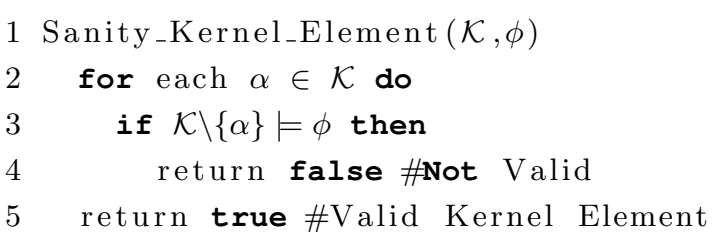

Listagem 4.5: Algoritmo para verificação de um elemento do conjunto kernel

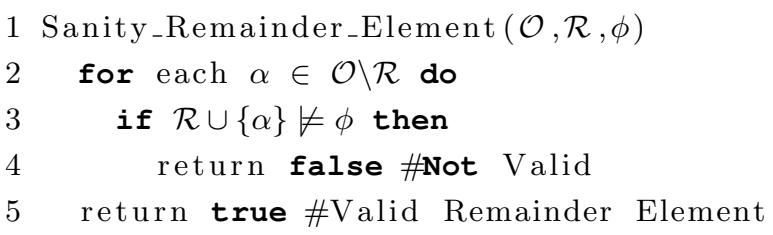

Listagem 4.6: Algoritmo para verificação de um elemento do conjunto resíduo

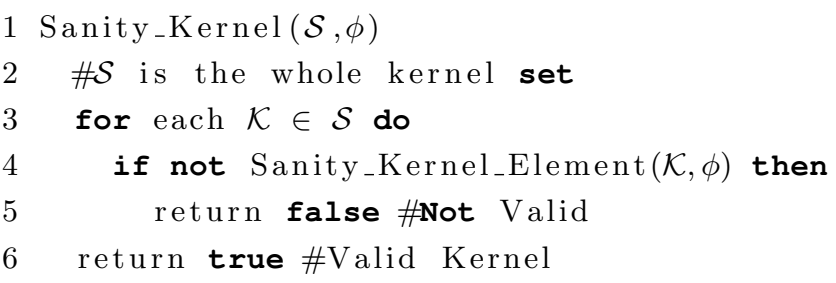

Listagem 4.7: Algoritmo para verificação do conjunto kernel

\subsubsection{Ambiente de Execução do Experimento}

Os experimentos foram executados no seguinte ambiente:

- CPU Intel XEON X5660 (12 núcleos)

\footnotetext{
${ }^{3}$ https://github.com/matthewhorridge/owlexplanation
} 
- 32 GB de RAM;

- Java v. 1.7.0_51;

- Linux Ubuntu 13.10 (Kernel 3.11.0-19-generic)

O java foi executado com os seguintes parâmetros: -Xms 2048m -Xmx16384m. Essas opções dão a máquina virtual 2 GB para o tamanho inicial da Heap, o que é um tamanho razoável, considerando que a configuração padrão é de 1/64 o tamanho da memória inteira; e 16 GB para o tamanho máximo da Heap.

Cada teste é realizando com um timeout, definido de forma empírica. Para o caso onde temos dados gerados automaticamente, cada teste teve um timeout de 45 segundos. No caso dos dados do BioPortal, utilizamos o timeout de 330 segundos por inferência indesejada.

\subsubsection{Dados Gerados}

Esse tipo de estratégia de geração de dados foi proposto por Resina et al. [RRW14]. O objetivo dessas estratégias de geração é estressar ao máximo esses algoritmos para construção de kernel/conjunto resíduo, com o intuito de tentar estabelecer um comportamento para o algoritmo de acordo com a natureza de suas entradas.

Utilizando essa estratégia de geração automática conseguimos ter controle sobre o tamanho do conjunto kernel/conjunto resíduo. Portanto, nossos casos de testes que fazem uso de ontologias geradas automaticamente se dividem em dois tipos, os que manipulam ontologias que possuem conjuntos kernel grandes e conjunto resíduo pequenos e o contrário, onde temos conjuntos resíduo grandes e kernel pequeno.

\subsubsection{Conjunto Kernel Pequeno - Resíduo Grande}

Essa família de ontologias possui as seguintes características estruturais:

$$
\mathcal{O}=\left\{\begin{array}{c}
A \sqsubseteq B_{1}, B_{1} \sqsubseteq C, \\
A \sqsubseteq B_{2}, B_{2} \sqsubseteq C, \\
\vdots \\
A \sqsubseteq B_{n}, B_{n} \sqsubseteq C
\end{array}\right\}
$$

Se calcularmos o conjunto kernel $\mathcal{K}$ e conjunto resíduo $\mathcal{R}$ para o axioma inferido $A \sqsubseteq C$ em $\mathcal{O}$ notamos que $|\mathcal{K}|=n$ e $|\mathcal{R}|=2^{n}$.

A construção desse caso de teste tem como objetivo construir ontologias com o maior número possível de ocorrências de kernel pequeno, de 2 elementos. Uma consequência direta dessa estratégia de geração é que os conjuntos resíduos restante podem utilizar qualquer combinação de remoção de um dos pares de cada kernel, daí o número elevado de opções.

Por exemplo, com $n=3$ nós obteríamos:

$$
\mathcal{K}=\left\{\begin{array}{c}
\left\{A \sqsubseteq B_{1}, B_{1} \sqsubseteq C\right\}, \\
\left\{A \sqsubseteq B_{2}, B_{2} \sqsubseteq C\right\}, \\
\left\{A \sqsubseteq B_{3}, B_{3} \sqsubseteq C\right\}
\end{array}\right\}
$$




$$
\mathcal{R}=\left\{\begin{array}{l}
\left\{A \sqsubseteq B_{1}, A \sqsubseteq B_{2}, A \sqsubseteq B_{3}\right\}, \\
\left\{A \sqsubseteq B_{1}, B_{2} \sqsubseteq C, A \sqsubseteq B_{3}\right\}, \\
\left\{A \sqsubseteq B_{1}, A \sqsubseteq B_{2}, B_{3} \sqsubseteq C\right\}, \\
\left\{A \sqsubseteq B_{1}, B_{2} \sqsubseteq C, B_{3} \sqsubseteq C\right\}, \\
\left\{B_{1} \sqsubseteq C, A \sqsubseteq B_{2}, A \sqsubseteq B_{3}\right\}, \\
\left\{B_{1} \sqsubseteq C, B_{2} \sqsubseteq C, A \sqsubseteq B_{3}\right\}, \\
\left\{B_{1} \sqsubseteq C, A \sqsubseteq B_{2}, B_{3} \sqsubseteq C\right\}, \\
\left\{B_{1} \sqsubseteq C, B_{2} \sqsubseteq C, B_{3} \sqsubseteq C\right\},
\end{array}\right\}
$$

Realizamos esse tipo de teste tanto com operadores para a construção de kernel quanto de conjunto resíduo. Nas próximas seções apresentaremos os resultados obtidos e comentários sobre os dados coletados.

4.4.6.1.1 Construção de Um Único Elemento do Conjunto Kernel A figura 4.5 mostra o comportamento dos operadores Black-box com entradas onde o conjunto kernel é pequeno e o resíduo é grande. É possível ver que as formas de expansão e encolhimento clássico exigem um maior número de chamadas ao mecanismo de inferências. A Figura 4.5 mostra como o número de chamadas ao mecanismo pode diminuir ao utilizarmos a divisão e conquista e a expansão utilizando a conectividade sintática

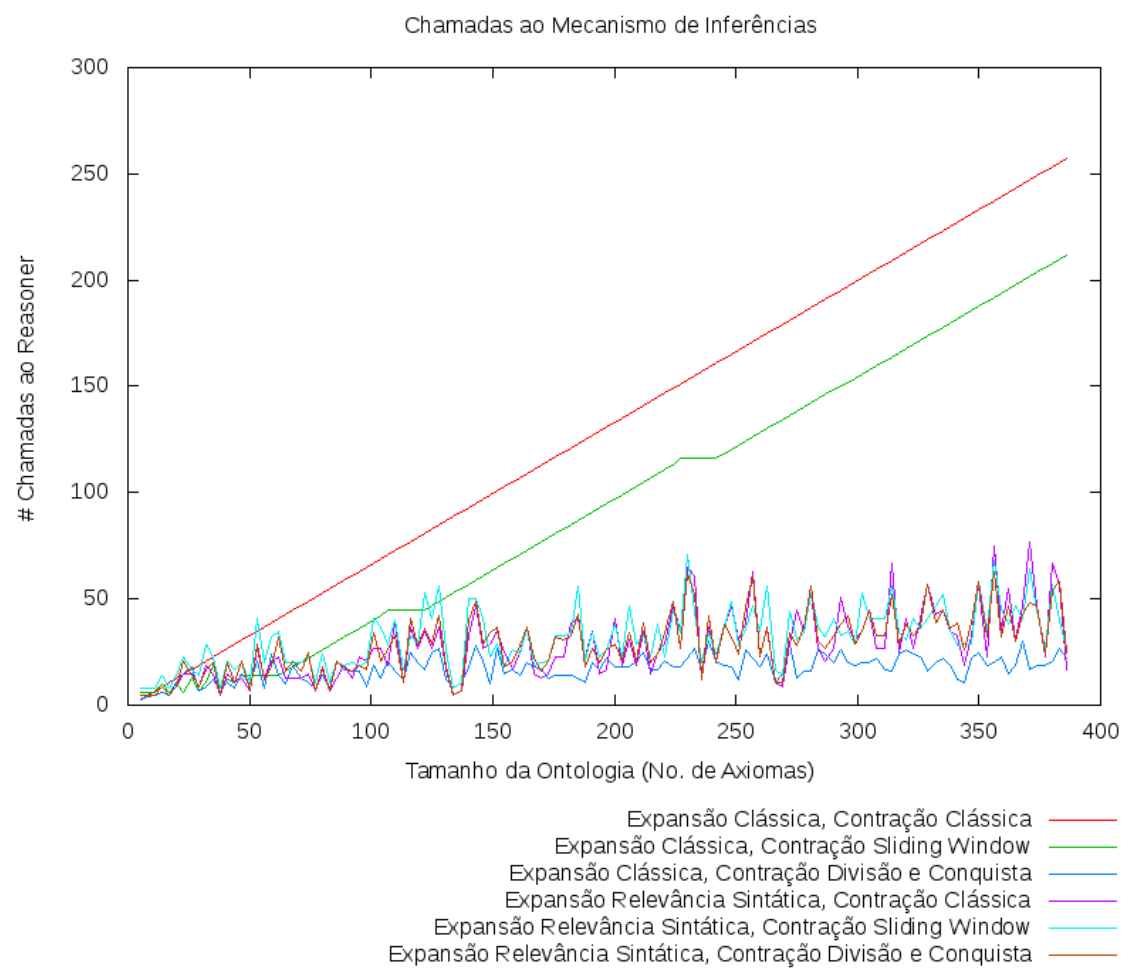

Figura 4.5: Comparativo entre algoritmos para construção do conjunto Kernel - Chamadas ao mecanismo de inferências

A Figura 4.6 mostra o tempo total de execução do algoritmo. A partir dela conseguimos ver que o desempenho melhorou com a adoção da relevância sintática e da divisão e conquista, que se manteve praticamente constante com o aumento do tamanho da base de conhecimento utilizado como entrada para o Black-box. 

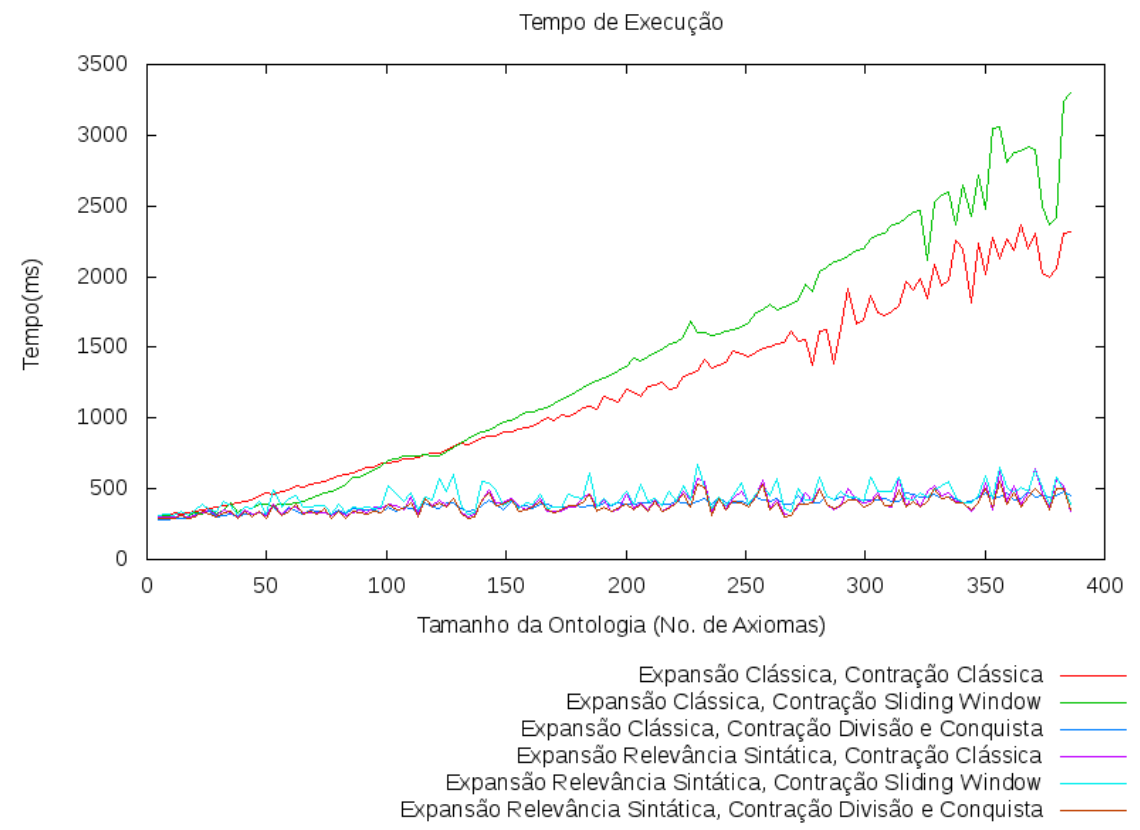

Figura 4.6: Comparativo entre algoritmos para construção do conjunto Kernel - tempo de execução

A Figura 4.7 mostra o uso da memória por parte da máquina virtual Java. É possível notar que as implementações que utilizam a expansão clássica junto do encolhimento clássico ou o encolhimento via janelas deslizantes apresentam um crescimento mais rápido no uso da memória. As demais implementações Black-box mantém um uso estável da memória.
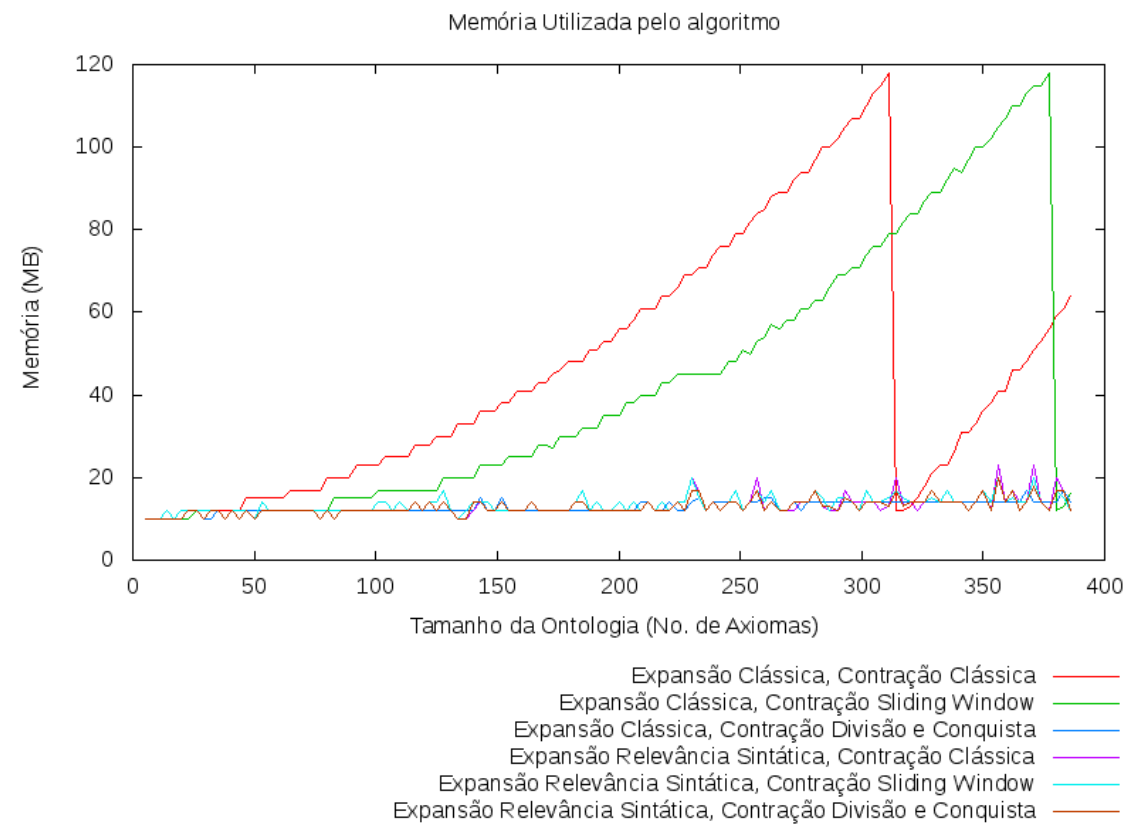

Figura 4.7: Comparativo entre algoritmos para construção do conjunto Kernel - uso de memória

4.4.6.1.2 Construção de Um Único Elemento do Conjunto Resíduo A Figura 4.8 mostra o comparativo entre o número de chamadas ao mecanismo de inferências. A Figura mostra que a expansão clássica, utilizando o encolhimento trivial, que retorna uma ontologia vazia rea- 
liza o menor número de chamadas ao mecanismo de inferências. Os resultados dos operadores que utilizam a expansão baseada em divisão e conquista eram esperados, uma vez que essa entrada é considerada o pior caso para esses algoritmos. A execução do algoritmo Black-box, composto pelo encolhimento trivial e o encolhimento utilizando divisão e conquista, com entrada $n=4$ pode ser vista no Exemplo 4.3.

Exemplo 4.3 Considere a ontologia :

$$
\begin{aligned}
\mathcal{O}=\{ & A \sqsubseteq B_{1}, \\
& B_{1} \sqsubseteq C, \\
& A \sqsubseteq B_{2}, \\
& B_{2} \sqsubseteq C, \\
& A \sqsubseteq B_{3}, \\
& B_{3} \sqsubseteq C, \\
& A \sqsubseteq B_{4}, \\
& \left.B_{4} \sqsubseteq C,\right\}
\end{aligned}
$$

É fácil ver que $\mathcal{O} \models A \sqsubseteq C$. O algoritmo de encolhimento trivial constrói a ontologia vazia. A expansão é responsável por adicionar o maior número de elementos removidos durante o encolhimento sem que a inferência indesejada se torne válida.

No primeiro passo, o algoritmo divisão e conquista divide $\mathcal{O}$ em $\mathcal{O}_{1}=\left\{A \sqsubseteq B_{1}, B_{1} \sqsubseteq C, A \sqsubseteq\right.$ $\left.B_{2}, B_{2} \sqsubseteq C\right\}$ e $\mathcal{O}_{2}=\left\{A \sqsubseteq B_{3}, B_{3} \sqsubseteq C, A \sqsubseteq B_{4}, B_{4} \sqsubseteq C\right\}$. Dessa divisão concluímos que $\mathcal{O}_{1} \models A \sqsubseteq C$ e $\mathcal{O}_{2} \models A \sqsubseteq C$. O algoritmo então é executado recursivamente em $O_{1}$ e $O_{2}$, obtendo $\mathcal{O}_{11}=\left\{A \sqsubseteq B_{1}, B_{1} \sqsubseteq C\right\}, \mathcal{O}_{12}=\left\{A \sqsubseteq B_{2}, B_{2} \sqsubseteq C\right\}, \mathcal{O}_{21}=\left\{A \sqsubseteq B_{3}, B_{3} \sqsubseteq C\right\}$ e $\mathcal{O}_{22}=\left\{A \sqsubseteq B_{4}\right.$, $\left.B_{4} \sqsubseteq C\right\}$. Novamente, é possivel inferir $A \sqsubseteq C$ de todas as sub-ontologias. O algoritmo faz então novamente a divisão obtendo $\mathcal{O}_{111}=\left\{A \sqsubseteq B_{1}\right\}, \mathcal{O}_{112}=\left\{B_{1} \sqsubseteq C\right\}, \mathcal{O}_{121}=\left\{A \sqsubseteq B_{2}\right\}, \mathcal{O}_{122}=$ $\left\{B_{2} \sqsubseteq C\right\}, \mathcal{O}_{211}=\left\{A \sqsubseteq B_{3}\right\}, \mathcal{O}_{212}=\left\{B_{3} \sqsubseteq C\right\}, \mathcal{O}_{221}=\left\{A \sqsubseteq B_{4}\right\}, \mathcal{O}_{222}=\left\{B_{4} \sqsubseteq C\right\}$.

As sub-ontologias criadas, individualmente não inferem $A \sqsubseteq C$. Essas sub-ontologias são os casos base da recursão. O algoritmo então, verifica se cada um dos axiomas individuais é consistente com a sub-ontologia criada pelo algoritmo até o momento. Considerando que a execução recursiva gerou as sub-ontologias na ordem listada acima, a execução do caso base da recursão resulta na adição das sub-ontologias $\mathcal{O}_{111}, \mathcal{O}_{121}, \mathcal{O}_{211}$ e $\mathcal{O}_{221}$.

O Exemplo 4.3 ilustra o pior caso de execução para o algoritmo de expansão baseada em divisão e conquista, uma vez que é necessário realizar a divisão da ontologia em axiomas individuais e chamadas ao mecanismo de inferências para cada uma das sub-ontologias unitárias.

A Figura 4.9 mostra o tempo total de execução do algoritmo. A partir dela conseguimos ver que o desempenho melhor foi obtido pelo algoritmo mais simples, que realiza o encolhimento trivial e a expansão utilizando a abordagem clássica. Nessa Figura também podemos notar que os piores desempenhos foram alcançados pelas abordagens de expansão divisão e conquista. Como explicamos para o número de chamadas ao mecanismo de inferências, essa natureza de dado de entrada representa o pior caso para esse algoritmo.

Esse tipo de entrada também é o pior cenário para o encolhimento utilizando a conectividade sintática, uma vez que todos os axiomas se relacionam com o axioma inferido. Assim, o encolhimento 

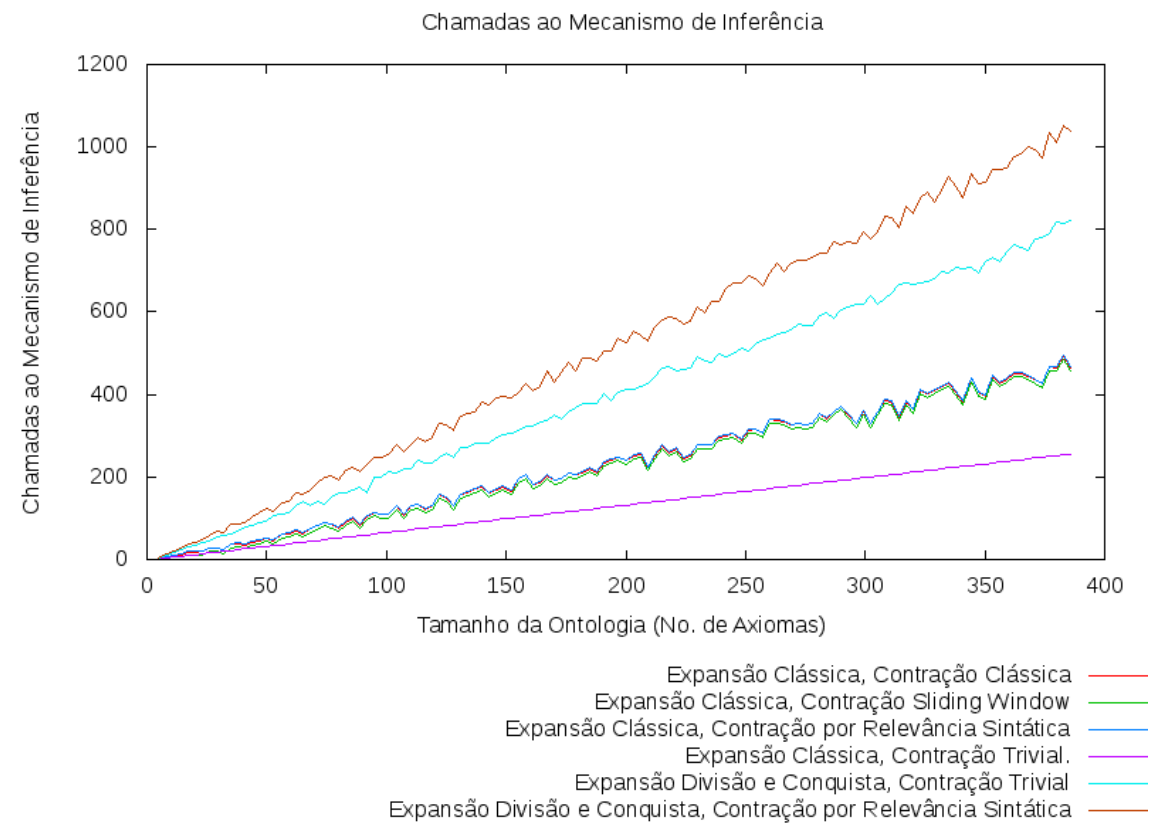

Figura 4.8: Comparativo entre algoritmos para construção do conjunto Resíduo - Chamadas ao mecanismo de inferências

remove todos os axiomas da ontologia, realizando chamadas ao mecanismo de inferência para cada uma das remoções. Por isso, a expansão utilizando divisão e conquista e o encolhimento utilizando a conectividade sintática são as piores escolhas de estratégias de otimização para esse tipo de dado de entrada.

A Figura 4.10 mostra o uso da memória por parte da máquina virtual Java. É possível notar que novamente, as implementações que utilizam a expansão baseada em divisão e conquista apresentam um crescimento mais rápido no uso da memória. Os decaimentos rápidos são resultados de chamadas ao coletor de lixo automático do Java. Nota-se que o Black-box implementado com o encolhimento trivial e expansão clássica se manteve por mais tempo crescendo antes da chamada ao coletor de lixo.

4.4.6.1.3 Comparativo Selecionamos três aspectos para comparar operadores Black-box para o conjunto Kernel e resíduo:

1. Chamadas ao mecanismo de inferências;

2. Tempo de execução;

3. Uso da Memória;

A Figura 4.11 mostra o número de chamadas ao mecanismo de inferências. A partir dela é possível ver que os algoritmos para cálculo de conjunto resíduo realizaram um número maior de chamadas ao mecanismo de inferências. Analisando melhor os dados, constatamos que, para esse padrão de entradas, construir um único conjunto resíduo é bem mais custoso computacionalmente do que construir um elemento único do kernel. Isso é confirmado pelas figuras 4.12 e 4.13, onde vemos que os operadores Black-box para a construção do conjunto resíduo levam mais tempo e também consomem mais memória para construir um único elemento. 


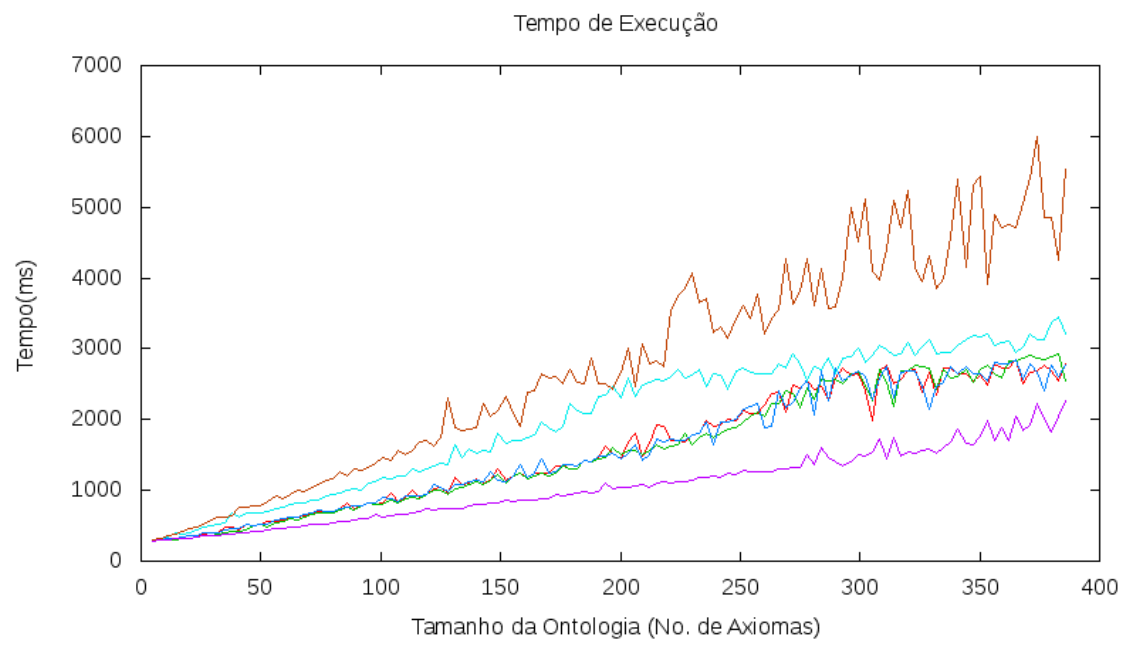

Expansão Clássica, Contração Clássica

Expansão Clássica, Contração Sliding Window Expansão Clássica, Contração por Relevância Sintática Expansão Divisão e Conquista, Contração Trivial Expansão Divisão e Conquista, Contração por Relevância Sintática

Figura 4.9: Comparativo entre algoritmos para construção do conjunto Resíduo - tempo de execução

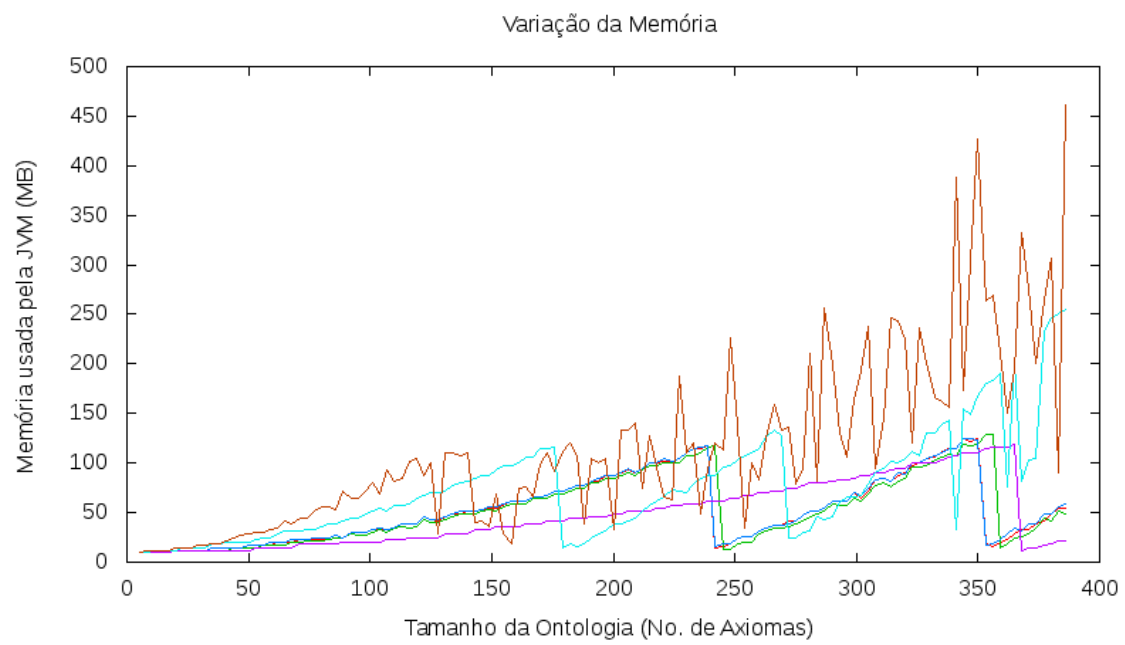

Expansão Clássica, Contração Clássica Expansão Clássica, Contração Sliding Window Expansão Clássica, Contração por Relevância Sintática Expansão Clássica, Contração Trivial. Expansão Divisão e Conquista, Contração Trivia Expansão Divisão e Conquista, Contração por Relevância Sintática

Figura 4.10: Comparativo entre algoritmos para construção do conjunto Resíduo - uso de memória 


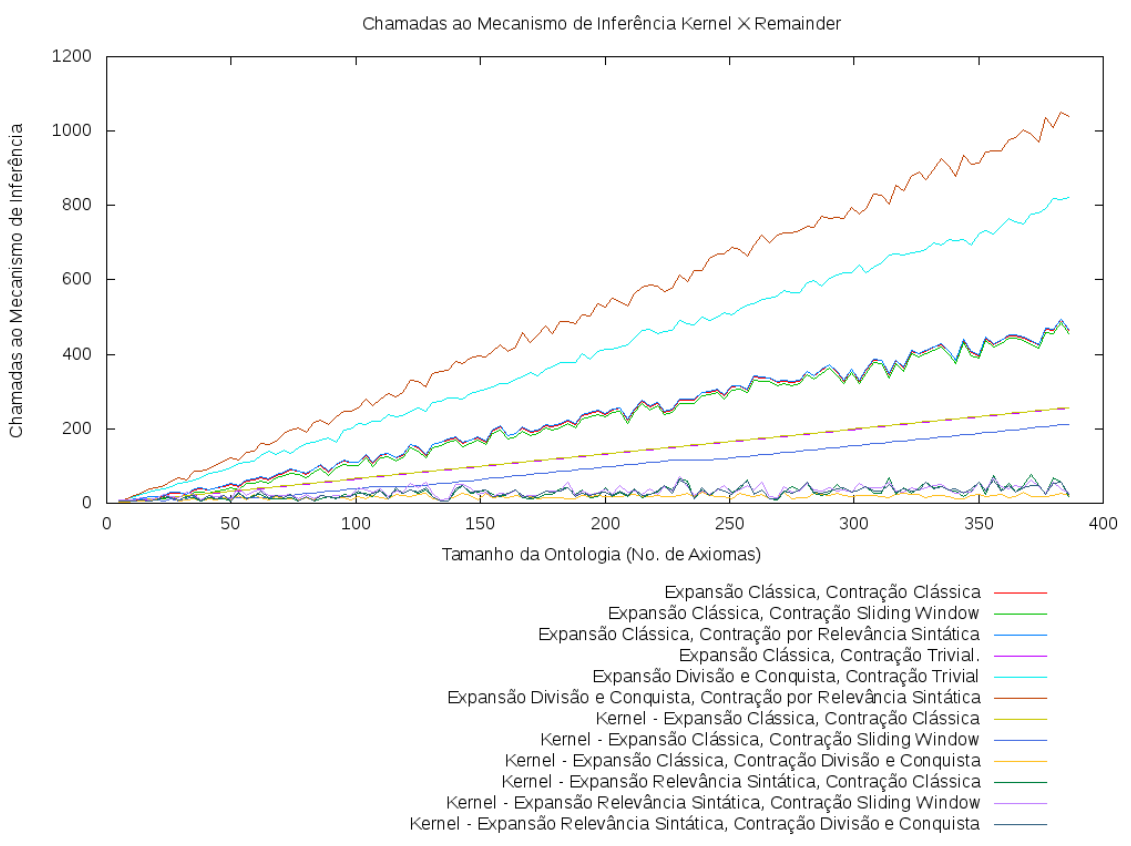

Figura 4.11: Comparativo entre algoritmos para construção dos conjuntos Kernel e Resíduo - Chamadas ao mecanismo de inferências

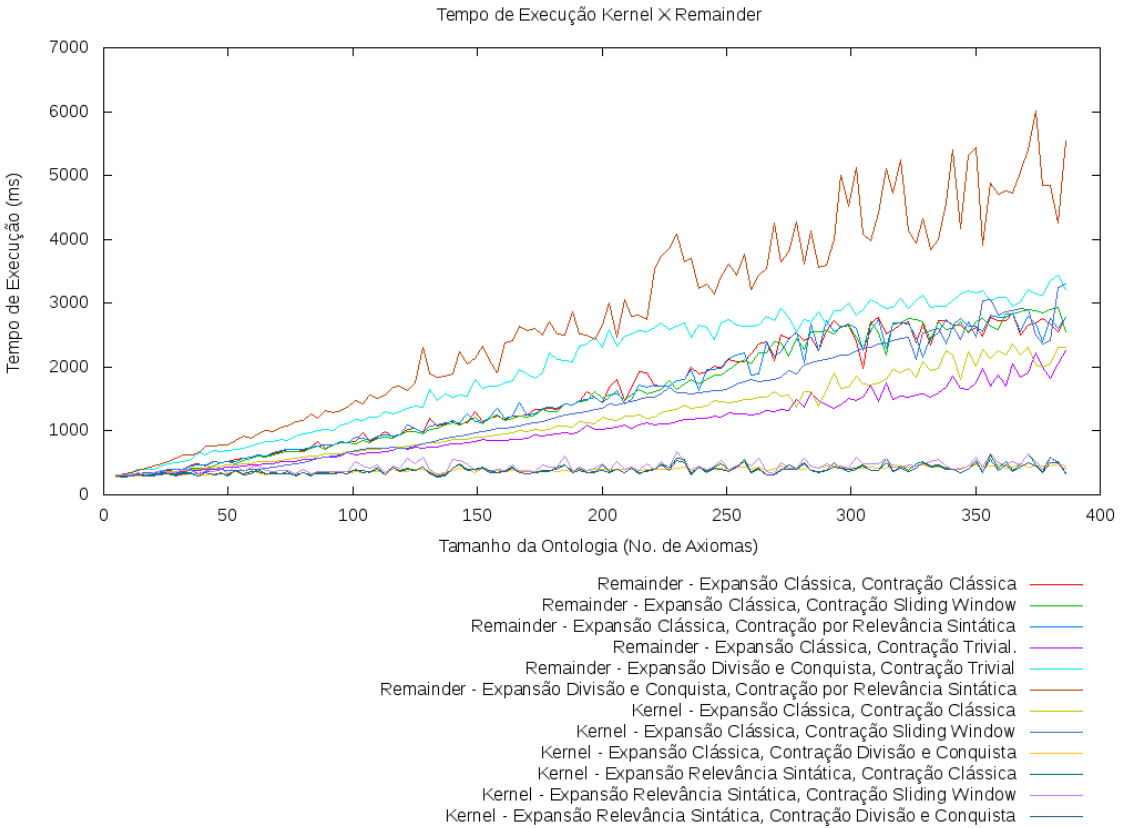

Figura 4.12: Comparativo entre algoritmos para construção dos conjuntos Kernel e Resíduo - Tempo de execução 


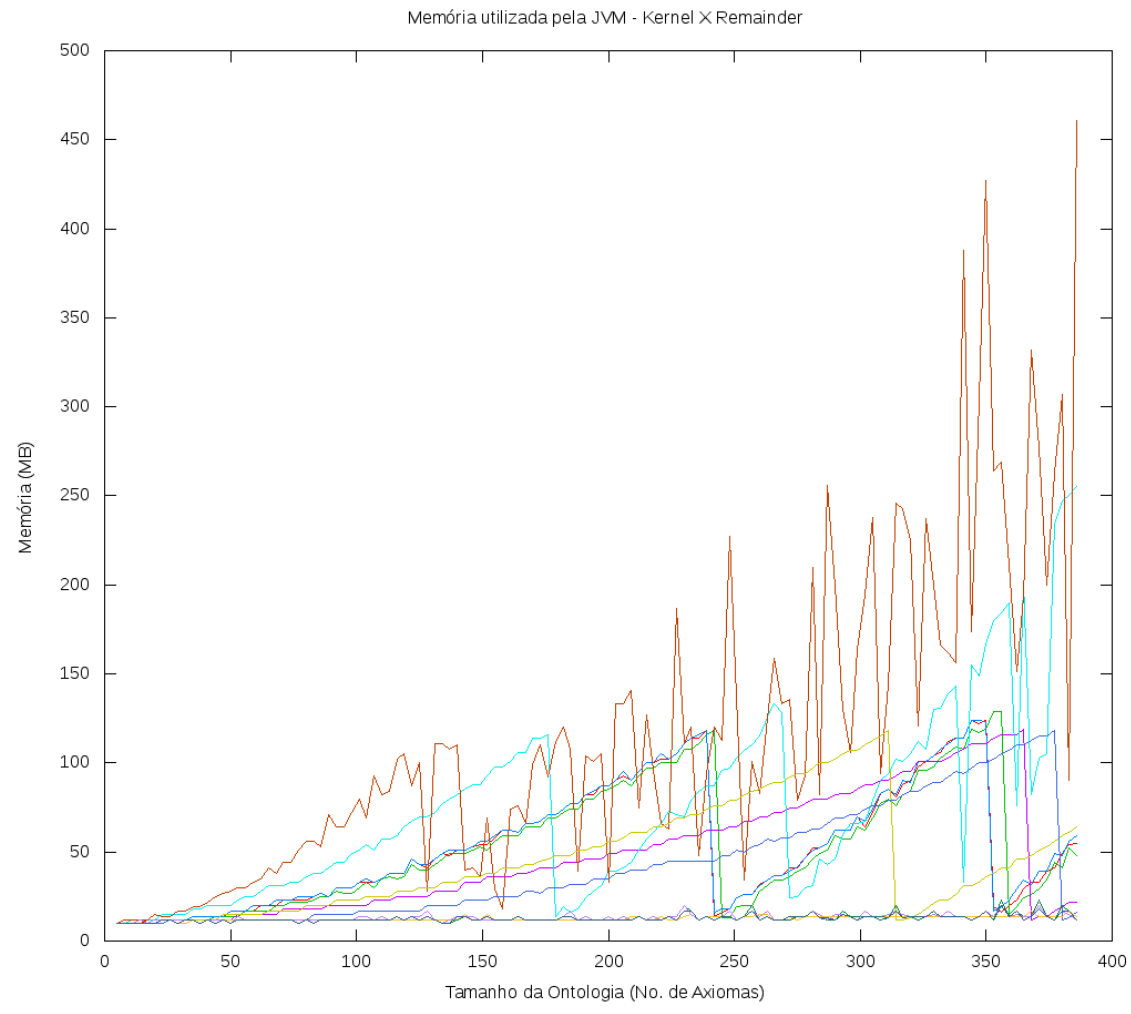

Remainder - Expansão Clássica, Contração Clássica Remainder - Expansão Clássica, Contração Sliding Window

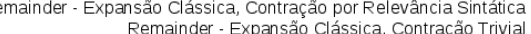
Remainder - Expansão Divisão e Conquista, Contração Trivial Remainder - Expansão Divisão e Conquista, Contraçãá por Relevância Sintática Kernel - Expansão Clássica, Contração Clássica Kernel - Expansão Clássica, Contração Sliding Window Kernel - Expansão Clássica, Contração Divisão e Conquista

Kernel - Expansão Relevância Sintática, Contraçáa Clássica Kernel - Expansão Relevância Sintática, Contração Sliding Window

Figura 4.13: Comparativo entre algoritmos para construção dos conjuntos Kernel e Resíduo - Uso da memória 


\subsubsection{Conjunto Kernel Grande - Resíduo Pequeno}

Essa família de ontologias possui as seguintes características estruturais:

$$
\mathcal{O}=\left\{\begin{array}{c}
\left(B \sqcup B^{\prime}\right)(a) \\
B \sqcup B^{\prime} \sqsubseteq B_{1}, B \sqcup B^{\prime} \sqsubseteq B_{1}^{\prime}, \\
B_{1} \sqcup B_{1}^{\prime} \sqsubseteq B_{2}, B_{1} \sqcup B_{1}^{\prime} \sqsubseteq B_{2}^{\prime}, \\
\vdots \\
B_{n-1} \sqcup B_{n-1}^{\prime} \sqsubseteq B_{n}, B_{n-1} \sqcup B_{n-1}^{\prime} \sqsubseteq B_{n}^{\prime}
\end{array}\right\}
$$

Se calcularmos o conjunto kernel $\mathcal{K}$ e conjunto resíduo $\mathcal{R}$ para o axioma inferido $\left(B_{n} \sqcup B_{n}^{\prime}\right)(a)$ em $\mathcal{O}$ notamos que $|\mathcal{K}|=2^{n}$ e $|\mathcal{R}|=n+1$.

Por exemplo, com $n=3$ nós obteríamos:

$$
\begin{gathered}
\mathcal{K}=\left\{\begin{array}{c}
\left\{\left(B \sqcup B^{\prime}\right)(a), B \sqcup B^{\prime} \sqsubseteq B_{1}, B_{1} \sqcup B_{1}^{\prime} \sqsubseteq B_{2}, B_{2} \sqcup B_{2}^{\prime} \sqsubseteq B_{3}\right\} \\
\left\{\left(B \sqcup B^{\prime}\right)(a), B \sqcup B^{\prime} \sqsubseteq B_{1}^{\prime}, B_{1} \sqcup B_{1}^{\prime} \sqsubseteq B_{2}, B_{2} \sqcup B_{2}^{\prime} \sqsubseteq B_{3}\right\} \\
\left\{\left(B \sqcup B^{\prime}\right)(a), B \sqcup B^{\prime} \sqsubseteq B_{1}, B_{1} \sqcup B_{1}^{\prime} \sqsubseteq B_{2}^{\prime}, B_{2} \sqcup B_{2}^{\prime} \sqsubseteq B_{3}\right\} \\
\left\{\left(B \sqcup B^{\prime}\right)(a), B \sqcup B^{\prime} \sqsubseteq B_{1}^{\prime}, B_{1} \sqcup B_{1}^{\prime} \sqsubseteq B_{2}^{\prime}, B_{2} \sqcup B_{2}^{\prime} \sqsubseteq B_{3}\right\} \\
\left\{\left(B \sqcup B^{\prime}\right)(a), B \sqcup B^{\prime} \sqsubseteq B_{1}, B_{1} \sqcup B_{1}^{\prime} \sqsubseteq B_{2}, B_{2} \sqcup B_{2}^{\prime} \sqsubseteq B_{3}^{\prime}\right\} \\
\left\{\left(B \sqcup B^{\prime}\right)(a), B \sqcup B^{\prime} \sqsubseteq B_{1}^{\prime}, B_{1} \sqcup B_{1}^{\prime} \sqsubseteq B_{2}, B_{2} \sqcup B_{2}^{\prime} \sqsubseteq B_{3}^{\prime}\right\} \\
\left\{\left(B \sqcup B^{\prime}\right)(a), B \sqcup B^{\prime} \sqsubseteq B_{1}, B_{1} \sqcup B_{1}^{\prime} \sqsubseteq B_{2}^{\prime}, B_{2} \sqcup B_{2}^{\prime} \sqsubseteq B_{3}^{\prime}\right\} \\
\left\{\left(B \sqcup B^{\prime}\right)(a), B \sqcup B^{\prime} \sqsubseteq B_{1}^{\prime}, B_{1} \sqcup B_{1}^{\prime} \sqsubseteq B_{2}^{\prime}, B_{2} \sqcup B_{2}^{\prime} \sqsubseteq B_{3}^{\prime}\right\}
\end{array}\right\} \\
\mathcal{O} \backslash\left\{\left(B \sqcup B^{\prime}\right)(a)\right\} \\
\mathcal{R}=\left\{\begin{array}{c}
\mathcal{O} \backslash\left\{B \sqcup B^{\prime} \sqsubseteq B_{1}, B \sqcup B^{\prime} \sqsubseteq B_{1}^{\prime}\right\}, \\
\mathcal{O} \backslash\left\{B_{1} \sqcup B_{1}^{\prime} \sqsubseteq B_{2}, B_{1} \sqcup B_{1}^{\prime} \sqsubseteq B_{2}^{\prime}\right\}, \\
\mathcal{O} \backslash\left\{B_{2} \sqcup B_{2}^{\prime} \sqsubseteq B_{3}, B_{2} \sqcup B_{2}^{\prime} \sqsubseteq B_{3}^{\prime}\right\},
\end{array}\right\}
\end{gathered}
$$

Realizamos esse tipo de teste tanto com operadores para a construção de kernel quanto de conjunto resíduo. Nas próximas seções listaremos os resultados obtidos e algumas impressões sobre esses resultados.

\subsection{Construção de Um Único Elemento do Conjunto Kernel A Figura 4.14 mos-} tra o comportamento dos operadores Black-box com entradas onde o conjunto kernel é grande e o resíduo pequeno. Para essa forma de entrada, o número de chamadas ao mecanismo de inferência cresce praticamente linearmente para todos as combinações de estratégias de expansão/encolhimento. Dessa forma, em termos de chamadas ao mecanismo de inferências, a utilização de heurísticas de otimização que se mostraram eficientes para entradas apresentadas na Seção 4.4.6.1, não se mostraram eficientes para os dados gerados utilizando a estratégia mostrada na Seção 4.4.6.2.

Um dado interessante a se notar na Figura 4.14, é que as estratégias que utilizam divisão e conquista para o encolhimento que tiveram os melhores resultados em termos de chamadas ao reasoner nas entradas utilizadas na Seção 4.4.6.1 obtiveram resultados piores do que os operadores que utilizaram o encolhimento clássico.

A Figura 4.15 mostra o tempo total de execução do algoritmo. A partir dela conseguimos ver que o desempenho melhor ainda é dos algoritmos que utilizam divisão e conquista. Esse dado é interessante, uma vez que esses operadores realizaram mais chamadas ao mecanismo de inferências 

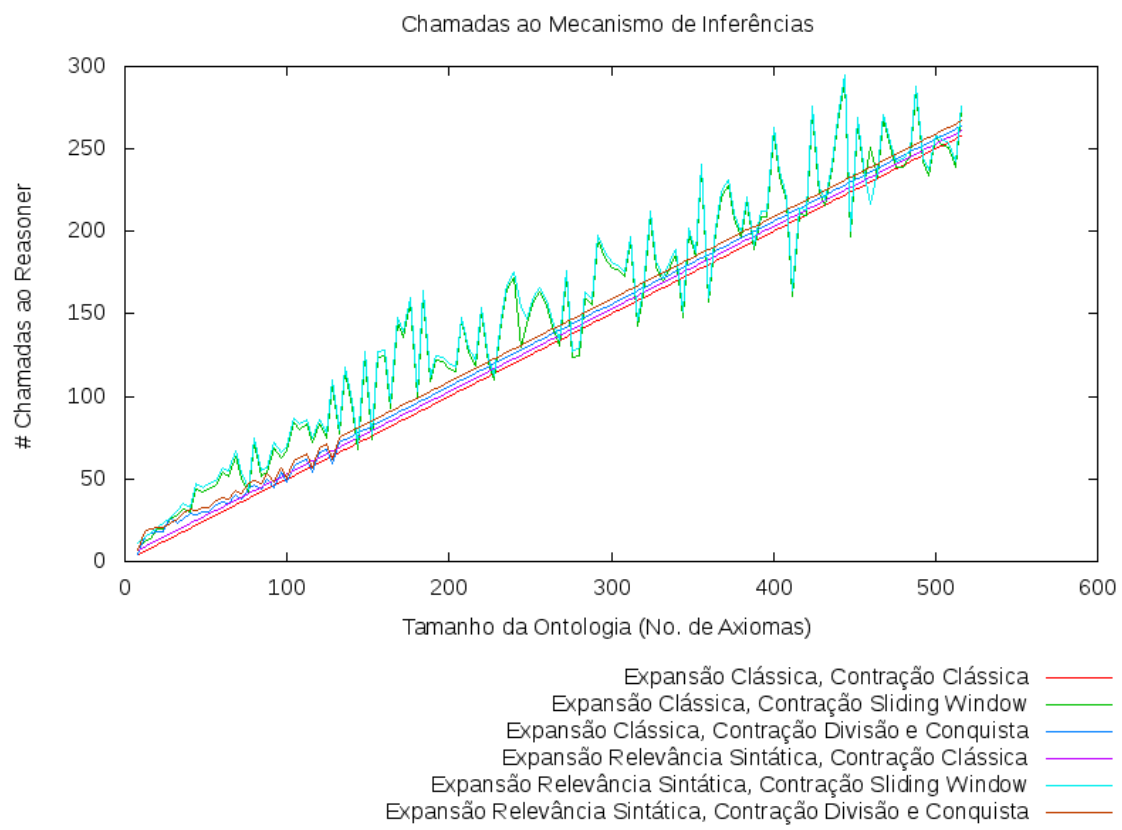

Figura 4.14: Comparativo entre algoritmos para construção do conjunto Kernel - Chamadas ao mecanismo de inferências

que os operadores que utilizaram encolhimento clássico.

Na Figura 4.15 também podemos ver que os piores desempenhos foram alcançados pelos algoritmos que utilizam janelas deslizantes durante a fase de encolhimento. Esse resultado está de acordo com o número de chamadas ao mecanismo de inferências. Esses operadores realizaram o maior número de chamadas e tiveram o pior desempenho.

A Figura 4.16 mostra que, com exceção dos algoritmos que utilizam janelas deslizantes - novamente os piores resultados, a utilização das heurísticas de otimização não resultou em melhorias no uso da memória.

\subsection{Construção de Um Único Elemento do Conjunto Resíduo A Figura 4.17} mostra o comparativo entre o número de chamadas ao mecanismo de inferências. Nela é possível ver que a expansão clássica, utilizando o encolhimento por conectividade sintática realiza o menor número de chamadas. Na verdade, ambos operadores que utilizam o encolhimento por conectividade sintática apresentaram números pequenos de chamadas ao mecanismo de inferências.

Os resultados obtidos com esse experimento foram de encontro aos dados levantados na Seção 4.4.6.1 pois o operador que utiliza a expansão clássica e o encolhimento trivial apresentou o maior número de chamadas ao mecanismo de inferências.

A Figura 4.18 mostra o tempo total de execução do algoritmo. Os dados apresentados nessa figura confirmam o que foi mostrado na Figura 4.17. Novamente as abordagens que utilizaram o encolhimento via conectividade sintática obtiveram melhores resultados, com tempos quase lineares para entradas de tamanho crescente.

A Figura 4.19 mostra que os algoritmos que utilizam a expansão via conectividade sintática obtiveram melhores resultados na utilização da memória. Em contrapartida, o operador que apresentou o menor uso de memória nos testes realizados na Seção 4.4.6.1, o operador que utiliza o encolhimento trivial e a expansão clássica apresentou o pior resultado no que diz respeito ao uso 


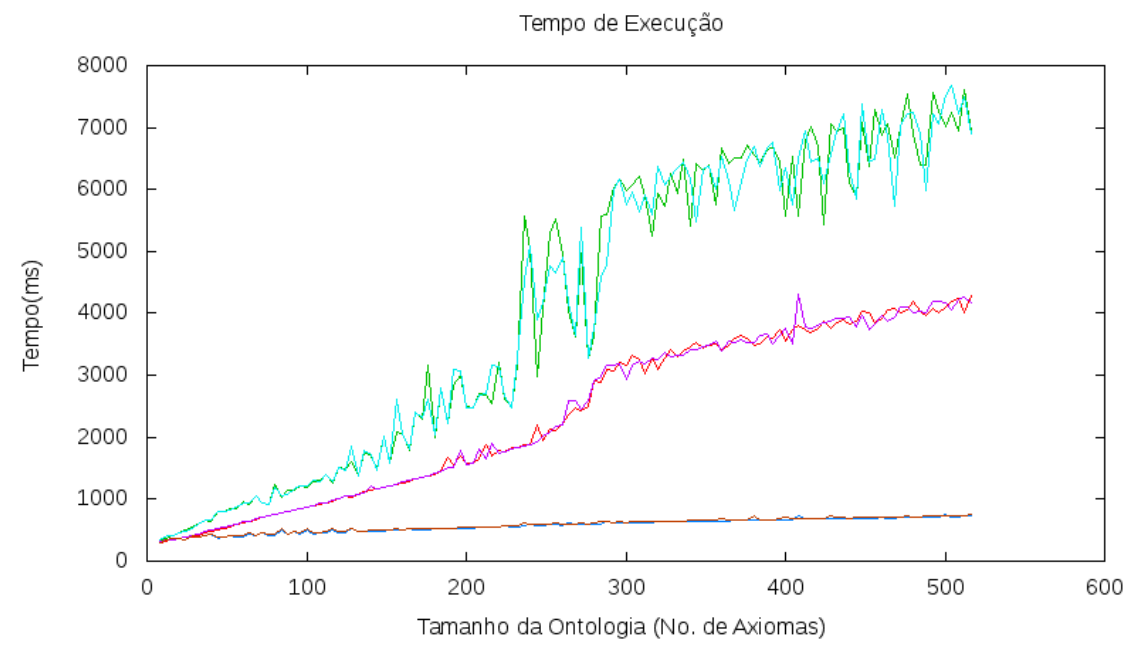

Expansão Clássica, Contração Clássica Expansão Clássica, Contração Sliding Window Expansão Clássica, Contração Divisão e Conquista Expansão Relevância Sintática, Contração Clássica

Expansão Relevância Sintática, Contração Sliding Window

Expansão Relevância Sintática, Contração Divisão e Conquista

Figura 4.15: Comparativo entre algoritmos para construção do conjunto Kernel - tempo de execução

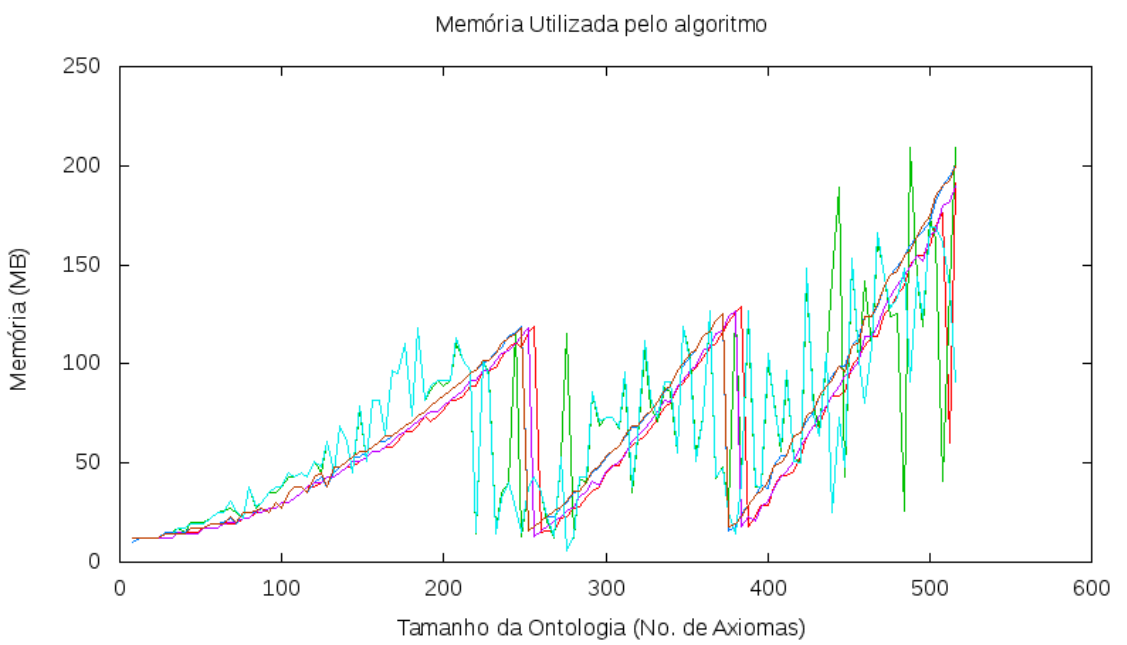

Expansão Clássica, Contração Clássica Expansão Clássica, Contração Sliding Window Expansão Clássica, Contração Divisão e Conquista Expansão Relevância Sintática, Contração Clássica Expansão Relevância Sintática, Contração Sliding Window Expansão Relevância Sintática, Contração Divisão e Conquista

Figura 4.16: Comparativo entre algoritmos para construção do conjunto Kernel - uso de memória 


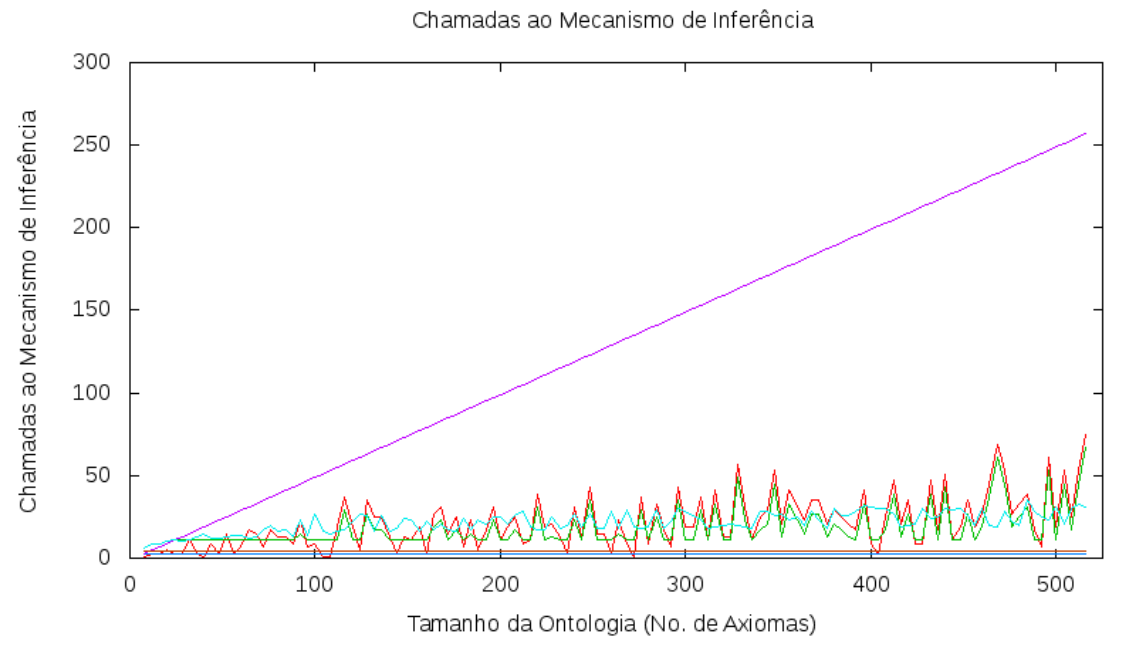

$$
\begin{array}{r}
\text { Expansão Clássica, Contração Clássica } \\
\text { Expansão Clássica, Contração Sliding Window } \\
\text { Expansão Clássica, Contração por Relevância Sintática } \\
\text { Expansão Clássica, Contração Trivial. } \\
\text { Expansão Divisão e Conquista, Contração Trivial } \\
\text { Expansão Divisão e Conquista, Contração por Relevância Sintática }
\end{array}
$$

Figura 4.17: Comparativo entre algoritmos para construção do conjunto Resíduo - Chamadas ao mecanismo de inferências
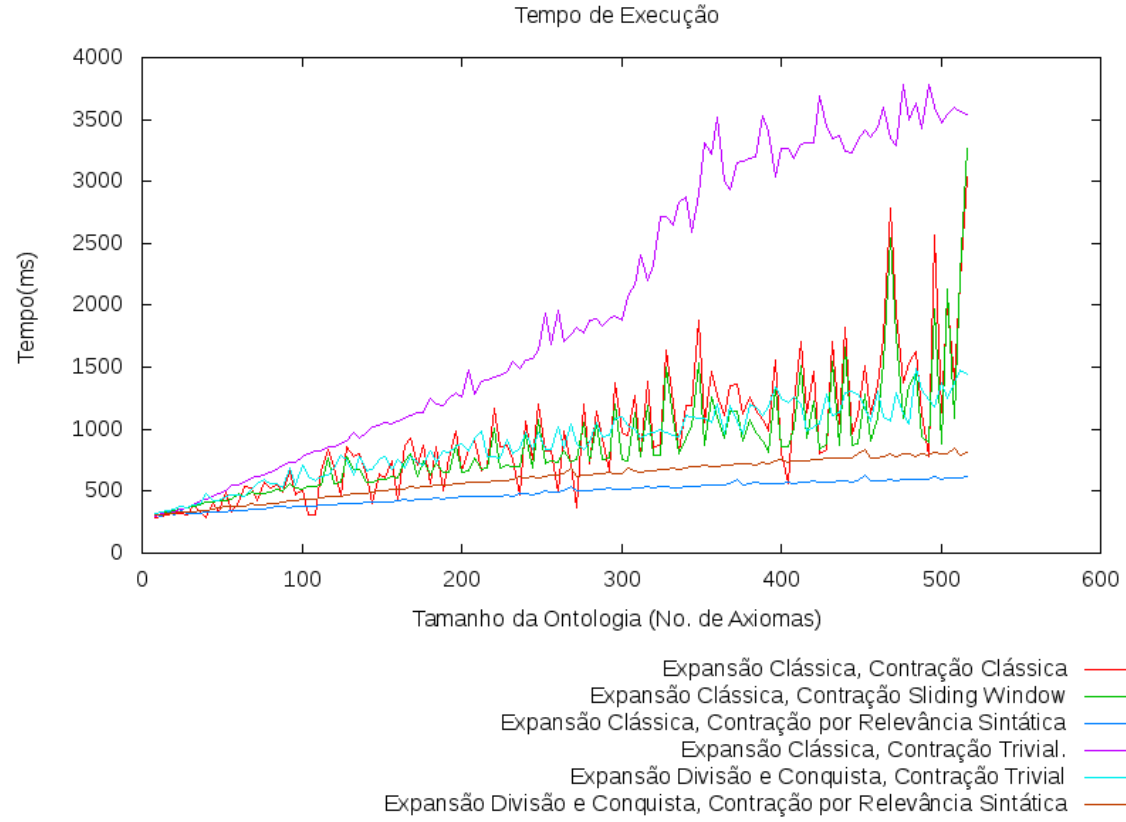

Figura 4.18: Comparativo entre algoritmos para construção do conjunto Resíduo - tempo de execução 
de memória.

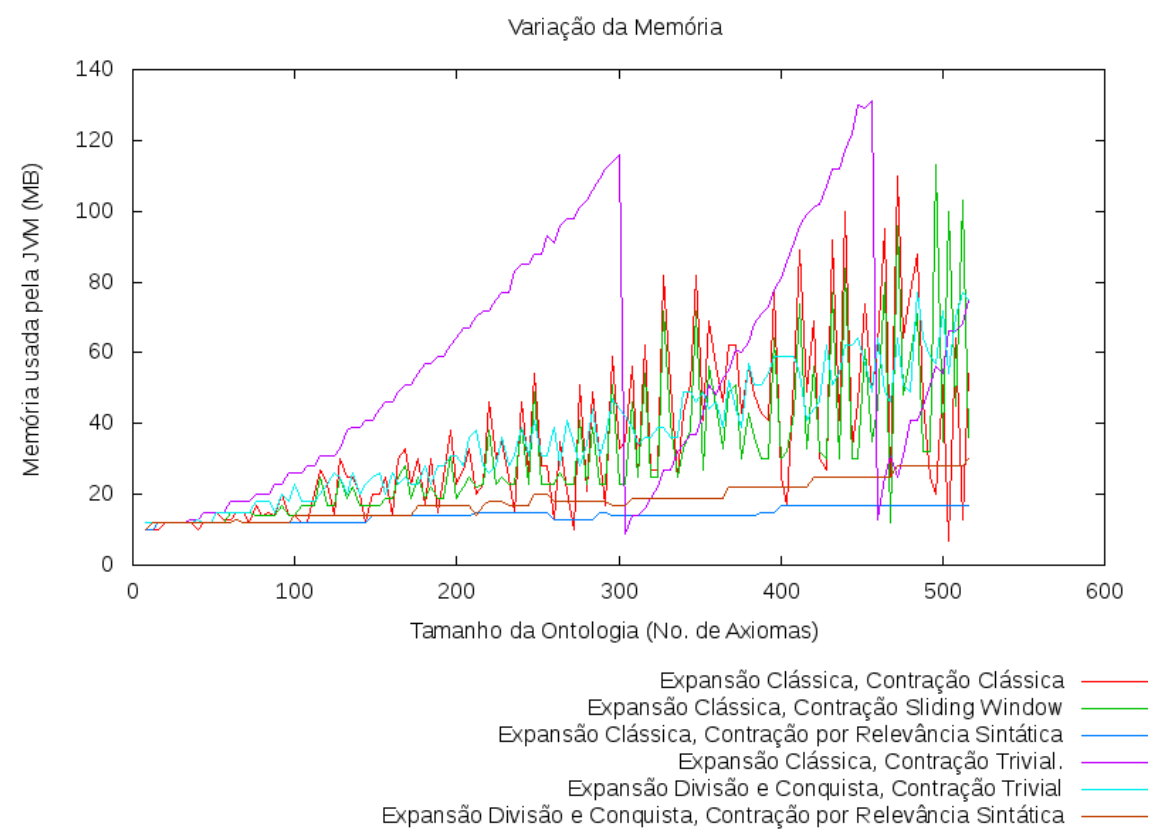

Figura 4.19: Comparativo entre algoritmos para construção do conjunto Resíduo - uso de memória

4.4.6.2.3 Comparativo Selecionamos três aspectos para comparar operadores Black-box para o conjunto Kernel e resíduo:

1. Chamadas ao mecanismo de inferências;

2. Tempo de execução;

3. Uso da Memória;

A Figura 4.20 mostra o número de chamadas ao mecanismo de inferências. A partir dela é possível ver que os algoritmos para cálculo de conjunto resíduo realizaram um número menor de chamadas ao mecanismo de inferências. Esse dado foi uma surpresa, uma vez que intuitivamente a construção do kernel pareça ser, computacionalmente, mais fácil por lidar com o critério da minimalidade. Porém, esses dados mostram que, em alguns casos, construir um conjunto resíduo é, computacionalmente mais fácil que o conjunto kernel. Vale a pena citar que não apenas os algoritmos que faziam usos de heurísticas apresentaram menores números de chamadas, mas todos os algoritmos se saíram melhores, com exceção da expansão clássica e encolhimento trivial, que apresentou desempenho aproximadamente igual aos Black-box para a construção de kernel.

A Figura 4.21 mostra a comparação entre as diversas abordagens Black-box. Nessa Figura podemos notar que o o melhor desempenho ainda foi de um operador Black-box para conjunto resíduo, porém o comportamento apresentado de crescimento é semelhante.

Assim como a Figura 4.20, A Figura 4.22 mostra um desempenho melhor por parte dos dois algoritmos de construção de conjuntos resíduos que utilizam a conectividade sintática para o encolhimento. 


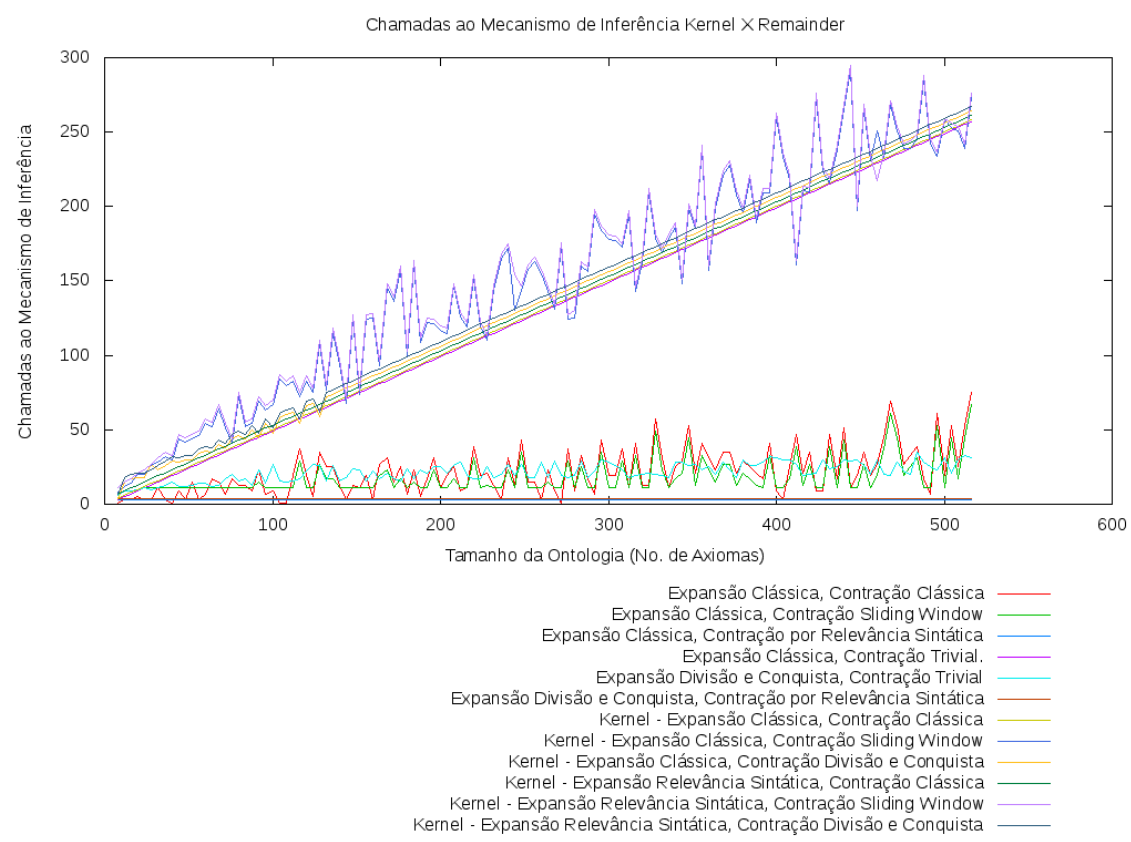

Figura 4.20: Comparativo entre algoritmos para construção dos conjuntos Kernel e Resíduo - Chamadas ao mecanismo de inferências

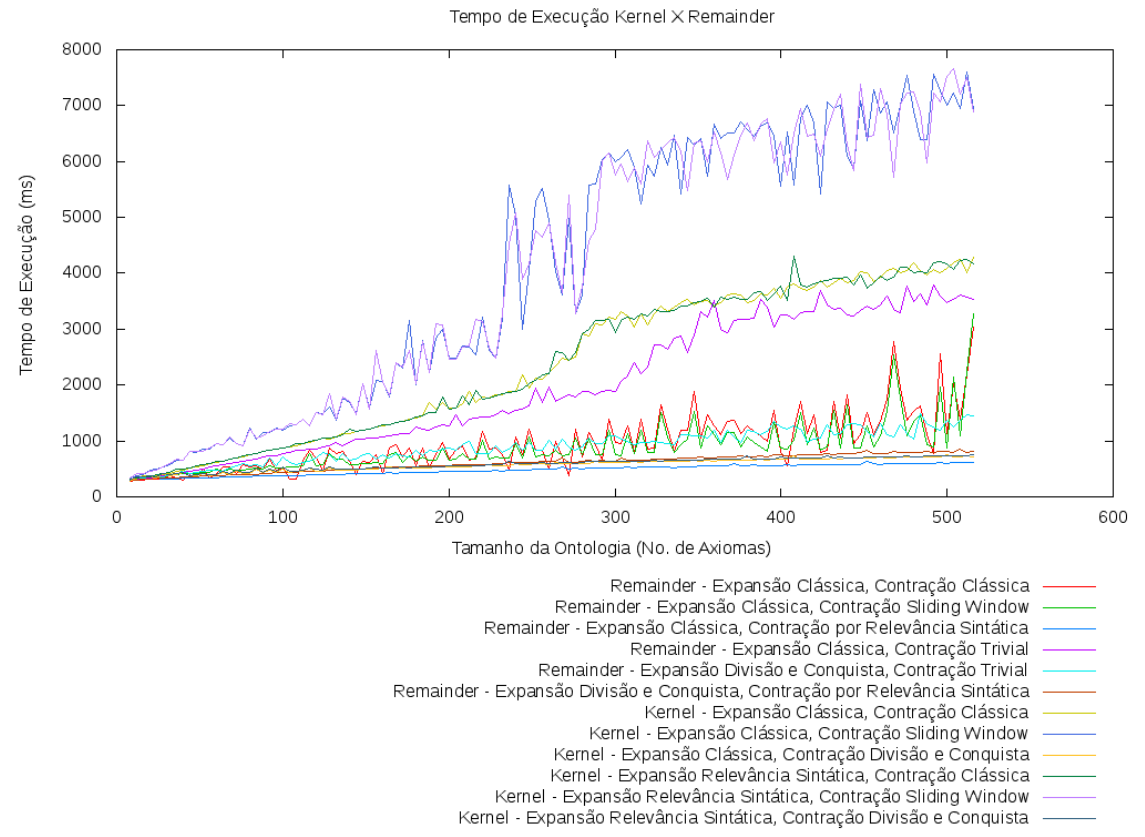

Figura 4.21: Comparativo entre algoritmos para construção dos conjuntos Kernel e Resíduo - Tempo de execução 


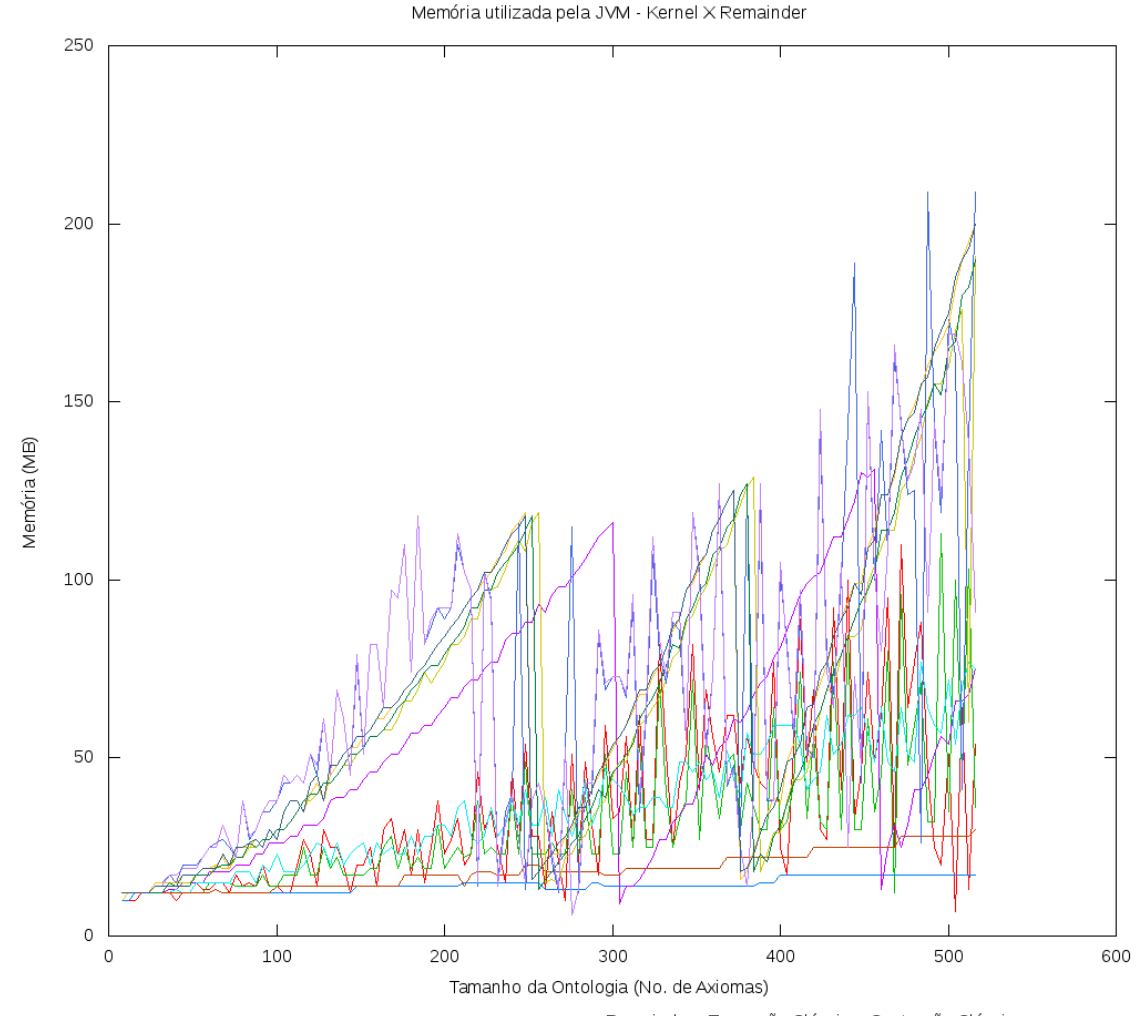

Remainder - Expansão Clássica, Contração Clássica

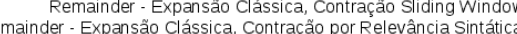

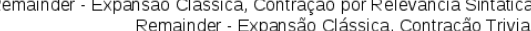
Remainder - Expansẫo Divisão e Conquista, Contração Trivial Remainder - Expansão Divisão e Conquista, Contraçãó por Relevância Sintática Kernel - Expansão clássica, Contração Clássica Kernel - Expansão Clássica, Contração Sliding Window Kernel - Expansão Clássica, Contração Divisão e Conquista

Kernel - Expansão Relevância Sintática, Contração Clássica

Kernel - Expansão Relevância Sintática, Contração Sliding Window

Kernet Expansão Relôn

Figura 4.22: Comparativo entre algoritmos para construção dos conjuntos Kernel e Resíduo - Uso da memória 


\subsubsection{Dados do BioPortal}

Realizamos um conjunto de experimentos com dados disponibilizados no portal agregador de ontologias do chamado BioPortal que foi desenvolvido pelo National Center for Biomedical Ontology - NCBO.

O objetivo desse Capítulo é mostrar que, para alguns casos envolvendo dados reais, o mesmo comportamento levantado durante os experimentos acontece, ou seja, é possível construir conjuntos resíduo tão rápido ou mais rápido que construir o conjunto kernel.

Os dados utilizados para o nosso experimento foram os mesmo disponibilizados por Horridge, em [Hor11]. O processo de adoção por dados do BioPortal se deu pelo fato do mesmo possuir uma interface HTTP REST para acesso a dados. Dessa forma foi possível baixar todas as ontologias disponíveis.

Horridge utilizou o seguinte processo de seleção de dados: primeiramente ele descartou as onto-

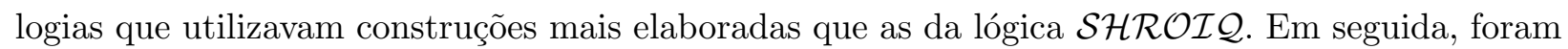
filtradas as ontologias descritas utilizando as linguagens OWL e OBO, a segunda sendo convertida para OWL. O autor selecionou, de um grupo de 219 ontologias, um conjunto de 72 ontologias que possuíam inferências não triviais, ou seja, dada a inferência $\alpha, \alpha$ é considerada não trivial caso $\mathcal{O} \backslash\{\alpha\} \models \alpha$, i.e., após remover o axioma da ontologia o mesmo ainda pode ser inferido.

Recolhemos o mesmo conjunto de dados - memória, tempo utilizado e número de chamadas ao mecanismo de inferência. Comparamos a construção de um único elemento do conjunto conflito com a construção de um único elemento do conjunto kernel e ao conjunto inteiro.

Selecionamos do conjunto de 72 ontologias, 51, cujo tamanho estava abaixo de $1 \mathrm{MB}$. Utilizamos esse critério pois o timeout estabelecido estava terminando o teste antes que a ontologia fosse carregada em memória. A implementação utilizada também faz a carga da mesma ontologia algumas vezes. Portanto, nossas soluções não estão otimizadas para tirar o maior proveito da OWLAPI, que o caso da ferramenta OWLExplanation plugin.

A Tabela 4.1 sumariza as ontologias utilizadas: 


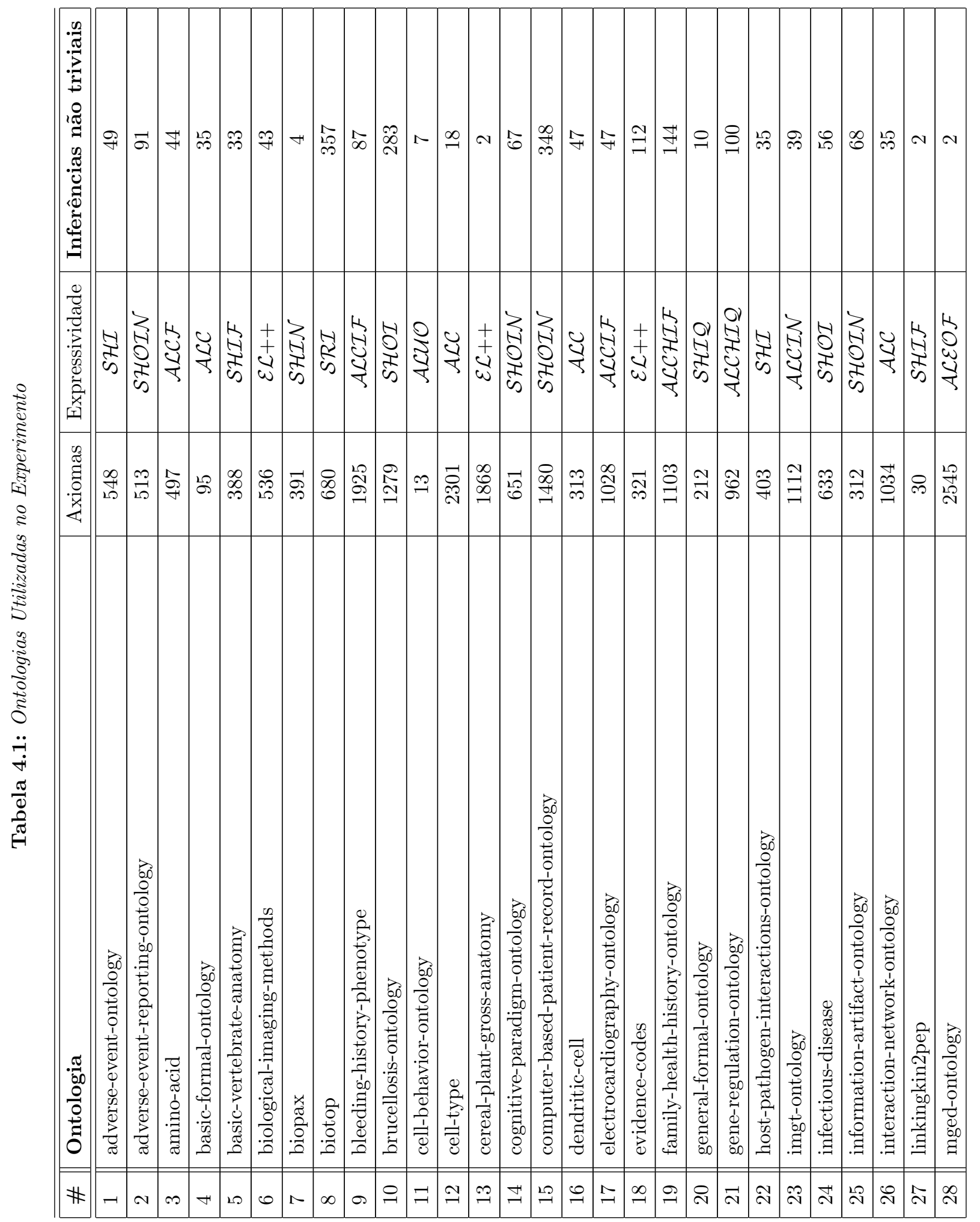




\begin{tabular}{|c|c|c|c|c|c|c|c|c|c|c|c|c|c|c|c|c|c|c|c|c|}
\hline-1 & $\stackrel{\infty}{\sharp}$ & $\begin{array}{l}\infty \\
\wp\end{array}$ & $\stackrel{\infty}{\sim}$ & $\stackrel{\infty}{\infty}$ & ชิ & 20 & $\overrightarrow{50}$ & 8 & กิ & $\vec{~} \vec{\infty}$ & $\Rightarrow 0$ & 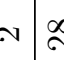 & \begin{tabular}{l|l}
0 \\
0
\end{tabular} & $\vec{J}$ & 아: & 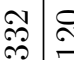 & 80 & 51 & \& & 0 \\
\hline 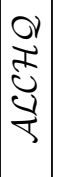 & $\begin{array}{l}O \\
\underset{T}{\alpha} \\
\infty\end{array}$ & $\frac{\pi}{\omega}$ & $\begin{array}{l}O \\
\frac{1}{N} \\
\frac{N}{2} \\
6\end{array}$ & $\begin{array}{l}0 \\
y \\
y \\
y \\
y\end{array}$ & 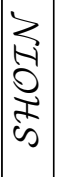 & $\begin{array}{l}0 \\
y \\
y \\
y\end{array}$ & $\underset{\substack{\alpha \\
\infty}}{\underset{j}{\alpha}}$ & 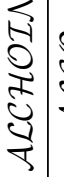 & $\begin{array}{l}0 \\
y \\
\frac{1}{S}\end{array}$ & 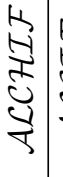 & 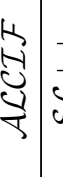 & \begin{tabular}{c|c}
+ & \\
+ \\
+ \\
$w$
\end{tabular} & 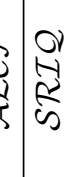 & $\begin{array}{l}\underset{x}{\alpha} \\
\infty\end{array}$ & 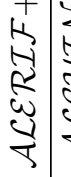 & 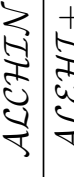 & 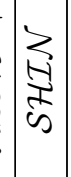 & $\left|\begin{array}{l}0 \\
- \\
\frac{2}{5} \\
\vec{y} \\
y\end{array}\right|$ & $\underset{\infty}{\grave{\alpha}}$ & $\begin{array}{l}+ \\
+ \\
\omega \\
\omega\end{array}$ \\
\hline 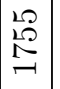 & 峉 & $\underset{8}{\mathscr{B}}$ & $\stackrel{\leftrightarrow}{\stackrel{2}{\circ}}$ & 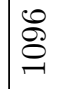 & $\mid \begin{array}{l}\infty \\
\mathscr{8} \\
\mathscr{\delta}\end{array}$ & $\stackrel{m}{\stackrel{m}{N}}$ & $\begin{array}{l}8 \\
0 \\
120\end{array}$ & స్ & 点 & 总 & $\left.18\right|_{\infty} ^{\infty}$ & 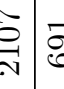 & 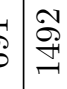 & 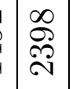 & 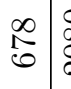 & \begin{tabular}{l|l}
$\stackrel{D}{D}$ \\
$\stackrel{\sim}{2}$
\end{tabular} & $\mid \begin{array}{l}20 \\
\mathscr{2} \\
\stackrel{2}{N}\end{array}$ & $\mid \begin{array}{l}\vec{D} \\
\stackrel{D}{*}\end{array}$ & $\begin{array}{l}\infty \\
\infty \\
\infty\end{array}$ & م્م \\
\hline 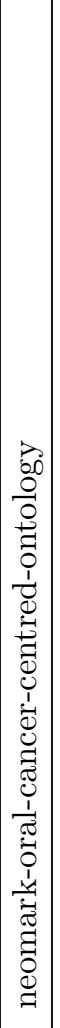 & 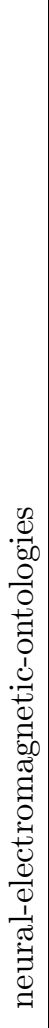 & 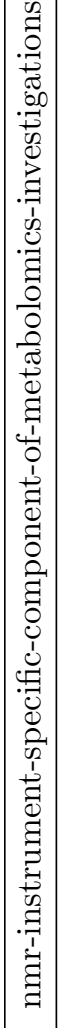 & $\begin{array}{l} \\
\\
\\
\\
8 \\
8 \\
8\end{array}$ & \begin{tabular}{|l}
0 \\
0 \\
0 \\
0 \\
0 \\
0 \\
0 \\
0 \\
0
\end{tabular} & 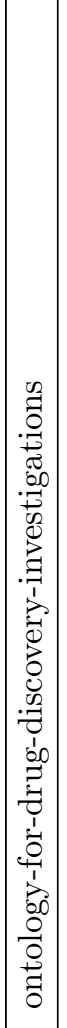 & 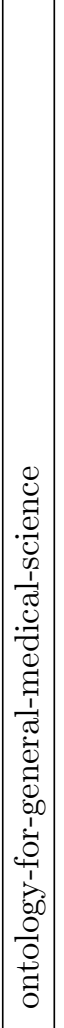 & 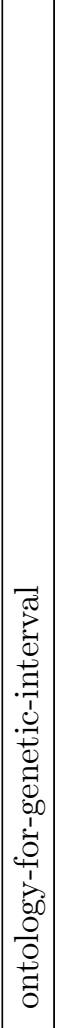 & 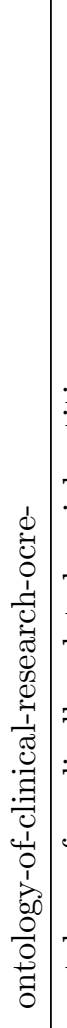 & 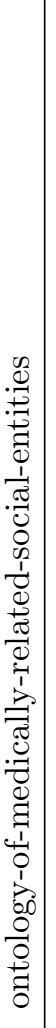 & 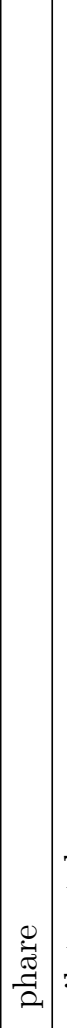 & 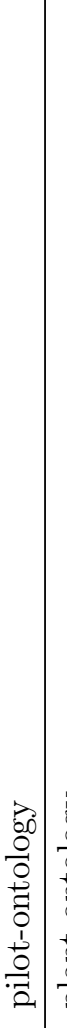 & 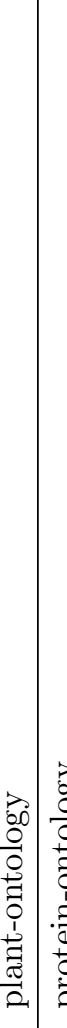 & 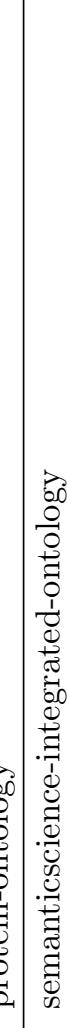 & 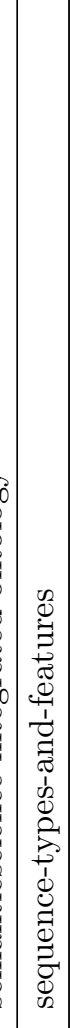 & 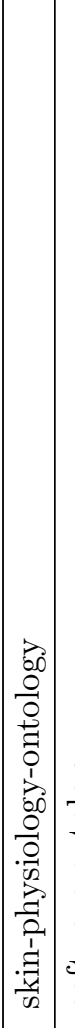 & 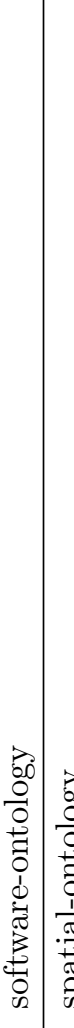 & 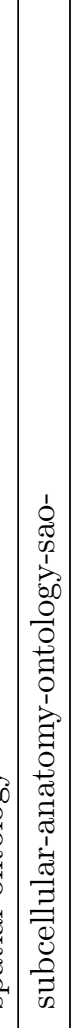 & 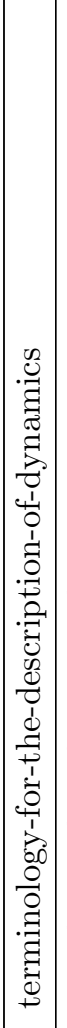 & 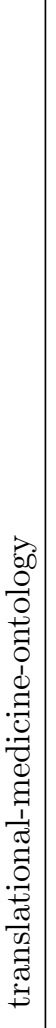 & 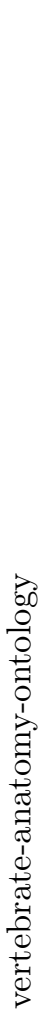 \\
\hline$\stackrel{\mathrm{N}}{ }$ & 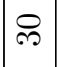 & $\vec{\infty}$ & ๓ै & $\dddot{m}$ & 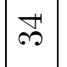 & 然 & $\mathscr{\infty}$ & $\widehat{\infty}$ & $\begin{array}{l}\infty \\
\infty \\
\infty\end{array}$ & $\dot{m}$ & 유: & $F$ & $\begin{array}{l}y \\
y\end{array}$ & $\exists$ & $\stackrel{2 S}{+2} 5$ & of & $\dot{H} \stackrel{\infty}{\rightarrow}$ & F & & 51 \\
\hline
\end{tabular}


Os dados coletados, por serem extensos se encontram disponíveis em http://www.ime.usp.br/ $\sim$ rmcobe/thesis/experiments. Aqui vamos apenas sumarizar o que foi coletado.

Utilizaremos símbolos apresentados na Tabela 4.2 para representar a combinação de estratégias utilizadas nos operadores para a construção do conjunto resíduo e kernel.

Tabela 4.2: Nomes dos operadores

\begin{tabular}{|l||l|}
\hline \hline Símbolo & Operador \\
\hline \hline ce_src & Encolhimento utilizando Conectividade Sintática - Expansão Clássica \\
\hline dace_src & Encolhimento utilizando Conectividade Sintática - Expansão via Divisão e Conquista \\
\hline dace_trc & Encolhimento Trivial - Expansão via Divisão e Conquista \\
\hline ce_trc & Encolhimento Trivial - Expansão Clássica \\
\hline ce_swc & Encolhimento via Janelas Deslizantes - Expansão Clássica \\
\hline ce_cc & Encolhimento Clássico - Expansão Clássica \\
\hline hr_sre_dacc & Abordagem utilizada por Horridge no owlexpanation plugin \\
\hline
\end{tabular}

\subsection{Chamadas ao Mecanismo de Inferência}

A Tabela 4.3 sumariza os dados coletados relativos ao número de chamadas ao mecanismo de inferência. A Tabela relaciona as ontologias utilizadas no experimento com o número de vezes nas quais utilizar cada operador foi mais eficiente em número de chamadas ao reasoner. Podemos ver que, dos 48 casos testados - nenhum dos operadores conseguiu construir conjuntos para os casos cell-behavior-ontology, linkingkin2pep e pilot-ontology dentro do timeout estipulado -, 19 ( 40\%) apresentaram melhores resultados (em número de cálculos dos conjuntos) na construção de um elemento do conjunto resíduo no lugar do conjunto kernel inteiro, o que corrobora com a nossa hipótese H3. Além disso, em todos os casos, nossas contribuições nos algoritmos para cálculo de um elemento do conjunto resíduo se mostraram eficazes, em especial a utilização da conectividade sintática durante o encolhimento, o que corrobora com nossa hipótese $\mathbf{H 1}$.

Um caso interessante que merece investigações futuras foi o da ontologia imgt-ontology, onde a conectividade sintática não apresentou os melhores resultados. Nesse caso, o uso do encolhimento trivial se mostrou uma melhor escolha. 


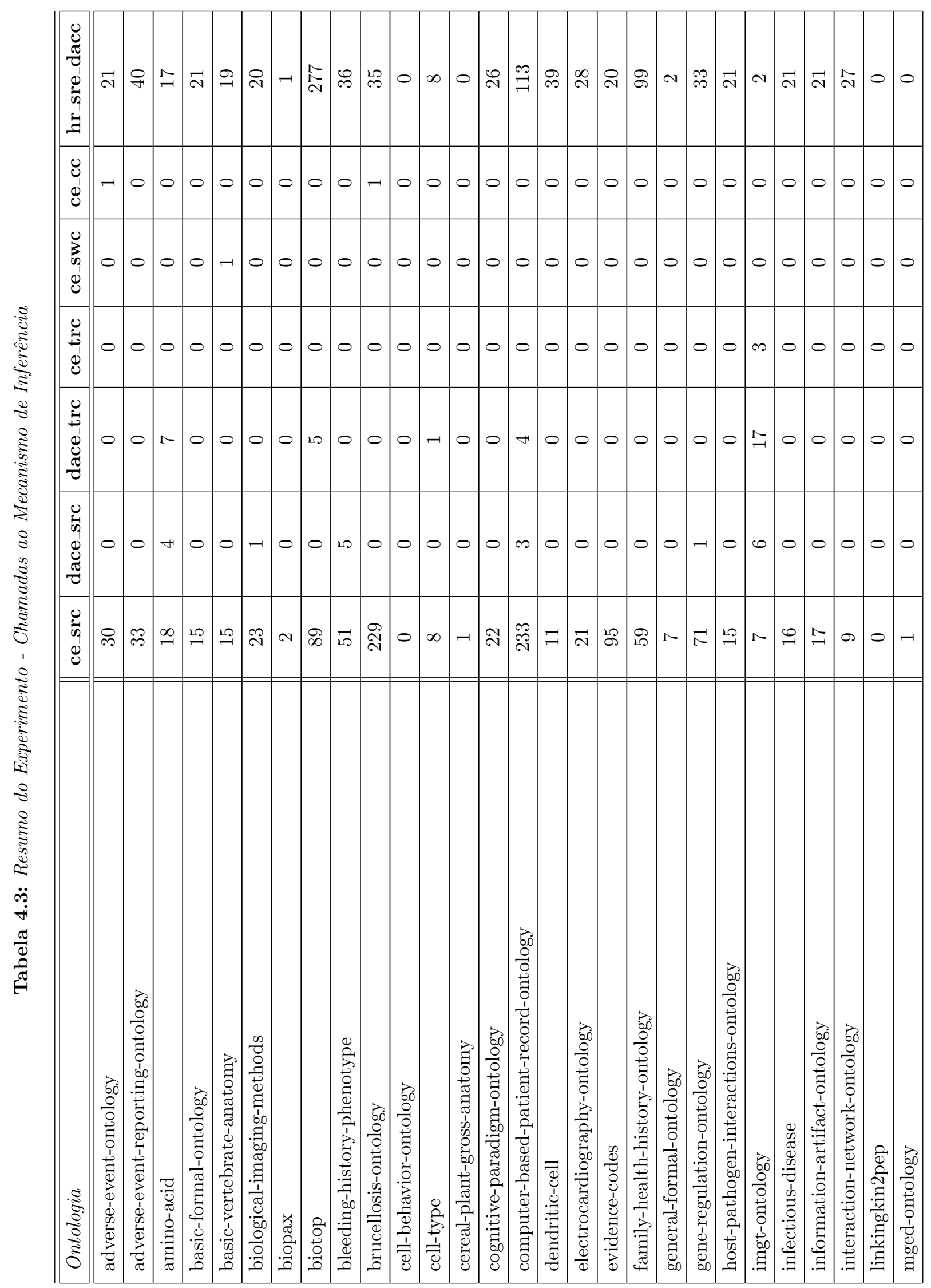




\begin{tabular}{|c|c|c|c|c|c|c|c|c|c|c|c|c|c|c|c|c|c|c|c|c|c|c|}
\hline 0 & $\stackrel{m}{=}$ & $\stackrel{\infty}{\sim}$ & $\infty$ & $\mathscr{\Omega}$ & ๓ै & $\stackrel{\rho}{\longrightarrow}$ & $\stackrel{N}{N}$ & 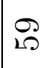 & $\stackrel{0}{-1}$ & 0 & 0 & 0 & $N$ & $\stackrel{100}{\circ}$ & $\begin{array}{l}\infty \\
\stackrel{10}{\sim} \\
\sim\end{array}$ & $\stackrel{H}{N}$ & $\underset{\sim}{*}$ & $\sqrt{20}$ & $\stackrel{12}{7}$ & $\stackrel{\sim}{\sim}$ & $\stackrel{\leftrightarrow}{\circ}$ & $N$ \\
\hline 0 & 0 & 0 & 0 & 0 & 0 & 0 & $N$ & 0 & 0 & 0 & 0 & 0 & 0 & 0 & 0 & -1 & 0 & 0 & 0 & 0 & 0 & 0 \\
\hline 0 & 0 & 0 & 0 & 0 & 0 & 0 & 0 & 0 & 0 & 0 & 0 & 0 & 0 & 0 & 0 & 0 & 0 & 0 & 0 & 0 & 0 & 0 \\
\hline 0 & 0 & 0 & 0 & 0 & 0 & 0 & 0 & 0 & 0 & 0 & 0 & 0 & 0 & 0 & 0 & 0 & 0 & 0 & 0 & 0 & 0 & 0 \\
\hline 0 & 0 & 0 & 0 & 0 & 0 & 0 & 0 & 0 & 0 & 0 & 0 & 0 & 10 & 0 & 0 & N & 0 & 0 & $\dashv$ & 0 & 0 & 0 \\
\hline 0 & 0 & 0 & 0 & 0 & 0 & 0 & 0 & 0 & 0 & 0 & 0 & 0 & $\sigma$ & 0 & 0 & 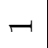 & 0 & 0 & N & 0 & 0 & 0 \\
\hline 0 & คి & $\exists$ & 0 & $a$ & 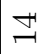 & 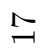 & $\stackrel{\curvearrowright}{\curvearrowright}$ & $\stackrel{\leftrightarrow}{\sim}$ & $\stackrel{\infty}{-1}$ & 令 & 0 & -1 & $\stackrel{\simeq}{\simeq}$ & $\stackrel{0}{0}$ & ৪ & $\stackrel{\mathcal{F}}{\sim}$ & $N$ & 8 & $\stackrel{\oplus}{\sim}$ & $\stackrel{\Omega}{\sim}$ & 등 & $\infty$ \\
\hline
\end{tabular}

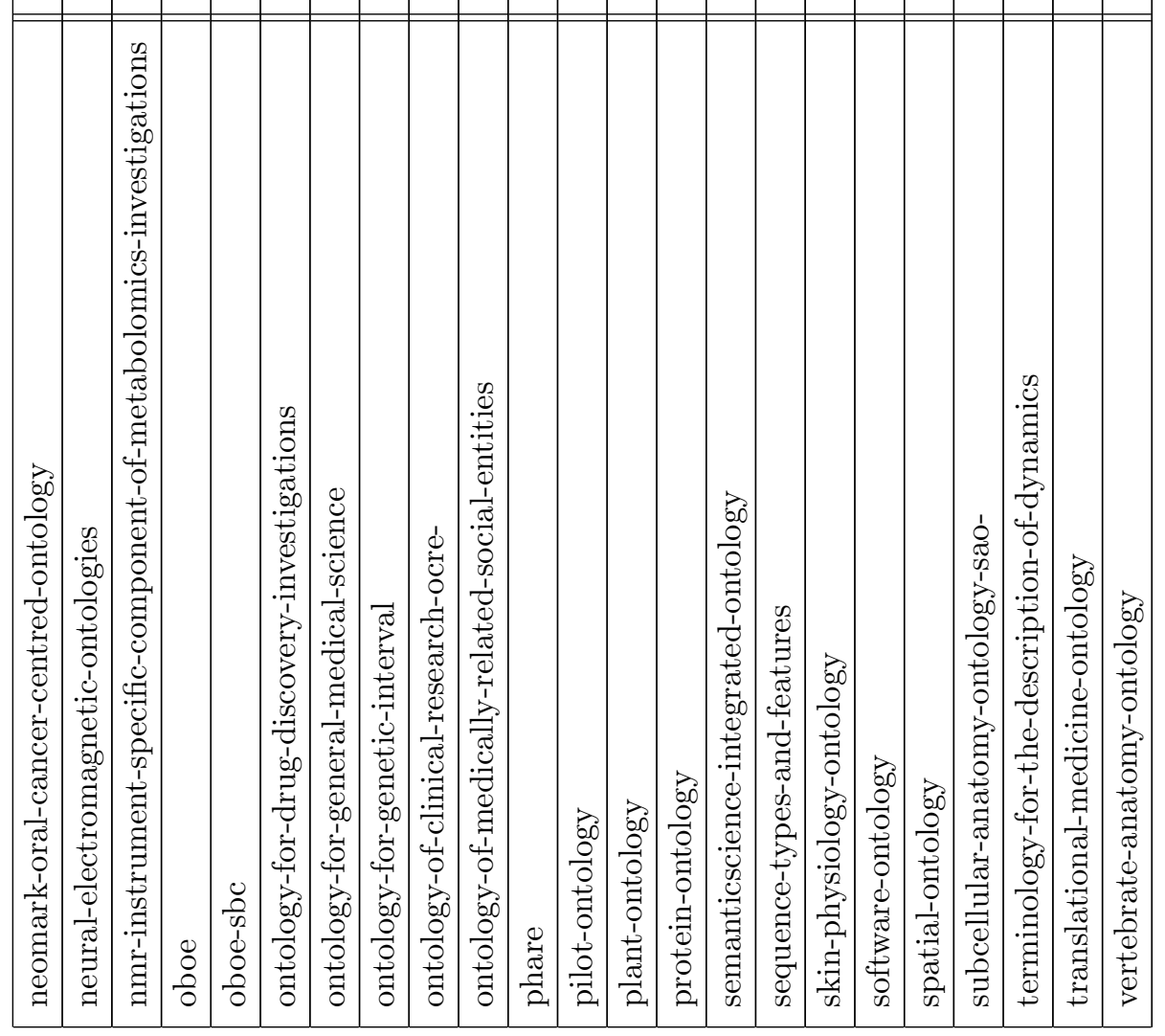




\subsection{Tempo de Execução}

A Tabela 4.4, assim como a apresentada na Seção 4.5 apresenta um resumo do experimento realizado com dados do BioPortal. Dessa vez estamos relacionando as ontologias utilizadas nos experimentos e o número de inferências nos quais cada um dos operadores se saiu melhor em termos de tempo de execução.

Esse experimento apontou que em grande maioria dos casos, a ferramenta OWLExplanation plugin plugin possui uma performance melhor em tempo de execução. O que pode se dar por conta do fato da mesma fazer melhor uso da OWLAPI.

Esses resultados apontam para oportunidades de melhorias em nossos algoritmos, e.g., em nossos algoritmos, criamos uma nova ontologia a cada checagem de inferência. Sabemos que essa criação é custosa, entretanto, um dos objetivos que buscávamos na nossa implementação era a simplicidade de código e a não dependência de uma API específica. Assim, lidamos com Ontologias como sendos conjuntos de axiomas, porém durante a checagem de inferência precisamos criar objetos específicos que são passados ao mecanismo de inferência.

A Tabela 4.4 mostra que em apenas um dos casos - imgt-ontology o cálculo de um único elemento do conjunto resíduo foi mais eficiente do que a criação de conjuntos kernel.

Um outro caso interessante foi o da ontologia amino-acid, onde a soma do número de inferências (14 - ce_src e 11 - dace_trc) onde construir o conjunto resíduo foi melhor, ultrapassou o número de vezes nas quais construir o conjunto kernel foi melhor. 


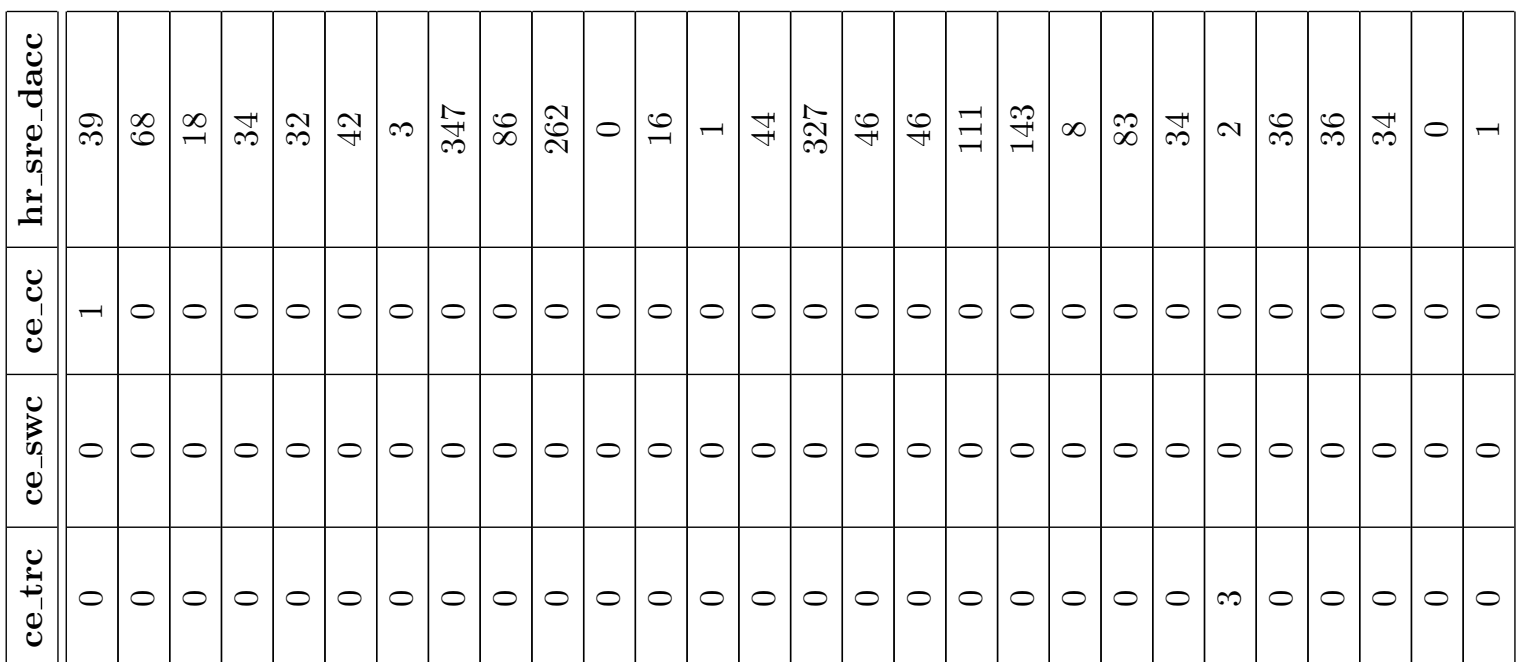

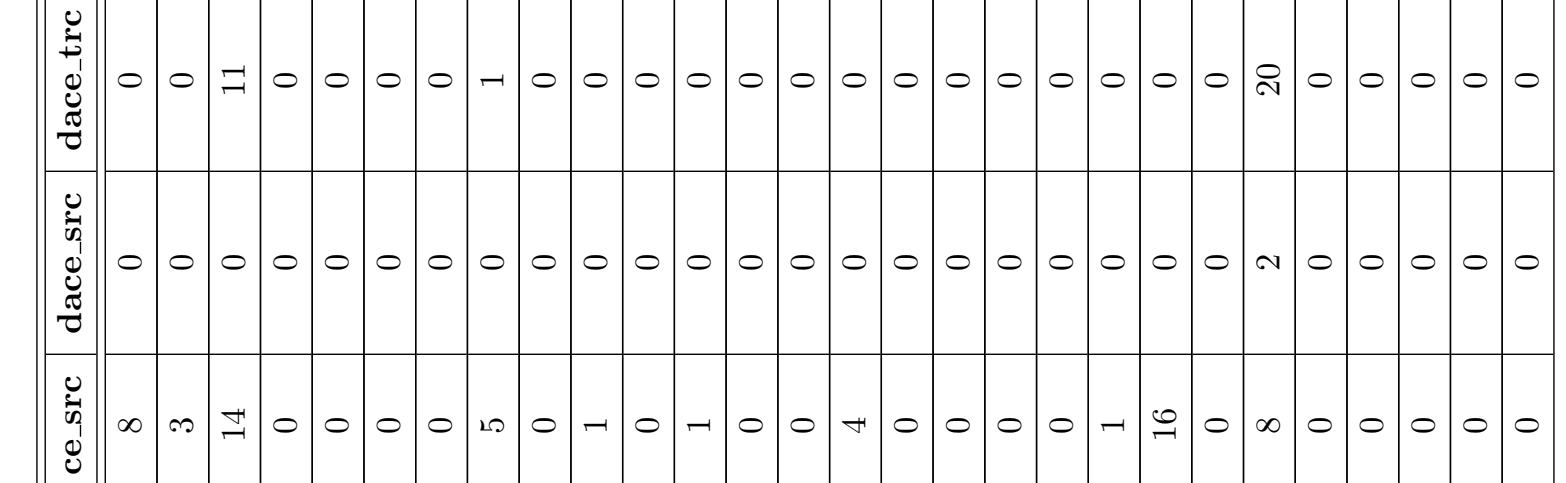




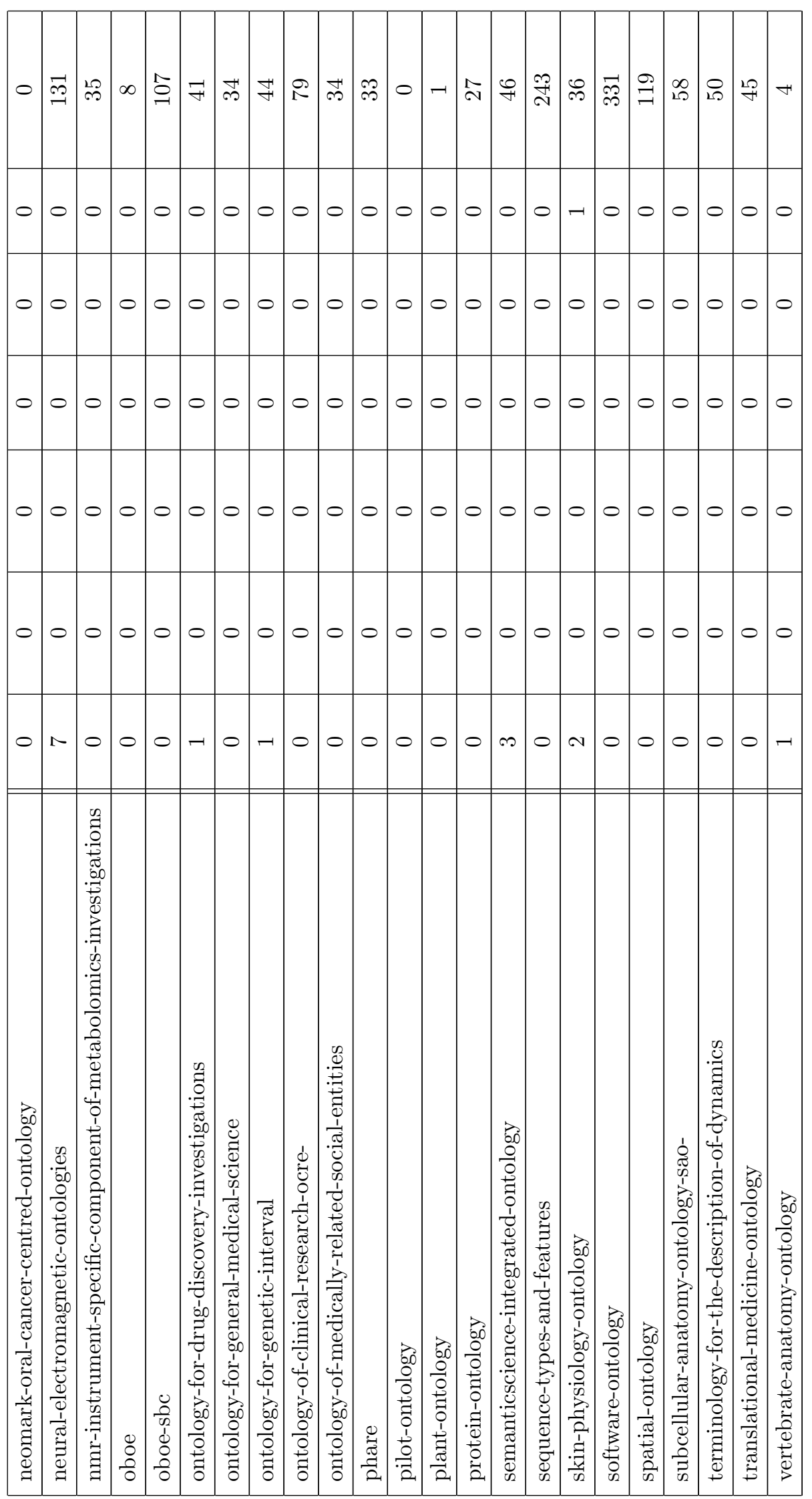




\subsection{Conclusão}

Nesse Capítulo mostramos os dados e análise dos experimentos realizados. Comprovamos que existem casos, teóricos (gerados) e reais nos quais calcular um conjunto resíduo pode ser feito de forma eficiente e o esforço para fazê-lo é igual se não menor ao esforço para a construção do conjunto kernel.

Gostaríamos de ressaltar alguns casos interessantes: na ontologia semanticscience-integratedontolog, em 3 inferências fomos capazes de construir apenas o conjunto resíduo. Casos semelhantes aconteceram a inferências em imgt-ontology (25 casos), biotop (4 casos), amino-acid (3 casos) e adverse-event-reporting-ontology (1 caso).

Na ontologia imgt-ontology, ocorreram 3 casos onde a criação de um conjunto resíduo utilizando a abordagem não otimizada, proposta por Resina et al. em [RRW14] foi melhor em termos de chamadas ao reasoner.

Na próxima Seção discutiremos as conclusões tiradas durante o desenvolvimento desse trabalho. Apresentaremos também pontos para melhorias e oportunidades para trabalhos futuros. 


\section{Capítulo 5}

\section{Considerações Finais}

\subsection{Considerações Finais e Contribuições}

Neste trabalho realizamos um extenso estudo sobre problemas decorrentes da fusão de ontologias, tanto do ponto de vista teórico quanto do ponto de vista prático. Aprendemos que muitas das ferramentas disponíveis podem ser classificadas em dois tipos: ferramentas para a depuração de ontologias e ferramentas que promovem reúso de ontologias. Infelizmente, também notamos que falta uma integração entre as duas capacidades.

As ferramentas que promovem o reúso, por exemplo, não lidam com problemas lógicos. Por outro lado, as que foram idealizadas para depuração de ontologias muitas vezes não possuem um mecanismo para fusão ou não realizam depuração de erros de modelagem - além dos lógicos inconsistências e incoerências.

Constatamos também que muitas das ferramentas se encontram descontinuadas ou desatualizadas, o que torna difícil reutilizar código e as ideias nelas implementadas, tendo em vista que a área de desenvolvimento de editores, APIs para manipulação e mecanismos de inferência é uma área que se desenvolve rápida e constantemente.

Com o objetivo de unificar tanto a área de modelagem de ontologias quanto a área de resolução de inconsistências/incoerências, centramos nosso trabalho no conceito de inferência indesejada, que pode ser um erro tanto lógico (inconsistência ou incoerência), ou um erro mais sutil, quando uma inferência não deveria ser válida dentro da modelagem de um domínio específico. Acreditamos que esse tipo de inferência pode ser o resultado de uma operação de fusão em ontologias. Tendo isso em mente, realizamos uma longa pesquisa de formas de lidar com inconsistências/incoerências assim como formas de entender essas inferências, calculando suas justificativas.

O resultado desse estudo nos levou a um conjunto de soluções bastante heterogêneo, onde cada uma lidava com o problema sob uma ótica diferente. Acreditamos que essas soluções podem e devem ser combinadas para um melhor resultado na resolução de conflitos advindos da fusão. Para agrupar essas abordagens distintas criamos uma classificação na forma de um processo onde cada fase agrupa estratégias de resolução de conflitos da mesma natureza. As fases do processo são encadeadas para que melhores resultados de fusões sejam possíveis.

Durante o desenvolvimento desse processo focamos, principalmente na depuração de inferências indesejadas em ontologias, sendo essa uma atividade chave para o reparo das mesmas. Propusemos uma estratégia para a separação do cálculo de conjuntos resíduos em expansão e contração. Propusemos diversas formas de otimização para cada uma dessas fases. Comprovamos que essas 
estratégias de otimização são eficazes e que de fato realizam um melhor aproveitamento de recursos (memória, chamadas ao mecanismo de inferências) e possuem, em média um tempo de execução melhor por meio de diversos testes com dados reais e dados gerados automaticamente.

Sendo assim, uma das contribuições mais significativas desse trabalho é trazer à luz os operadores de construção de conjuntos resíduos, muitas vezes não considerados no cálculo de reparos para inferências não desejadas, por serem considerados ineficientes.

Comparamos nossas novas operações para cálculo de elementos do conjunto resíduo com as otimizações mais comuns para a geração de elementos do conjunto kernel, utilizando dados gerados automaticamente e comprovamos que existem formas de ontologias nas quais a geração de um elemento do conjunto resíduo é mais eficiente, em termos de chamadas ao mecanismo de inferência, tempo de execução e memória do que a construção de um elemento do conjunto kernel, como pode ser visto na Seção 4.4.6.2.

Comparamos também os nossos operadores de construção de elementos do conjunto resíduo com a capacidade de geração de conjuntos kernel disponível na mais atual ferramenta que encontramos, o OWLExplanation plugin, utilizando dados de ontologias reais disponível no NCBO BioPortal. Nossos resultados sugerem que nem sempre a construção do conjunto kernel é mais eficiente do que a geração de um elemento do conjunto resíduo, principalmente no que diz respeito ao número de chamadas ao mecanismo de inferência, como pode ser visto na Seção 4.5. Infelizmente o mesmo não foi notado na questão tempo de execução. Entretanto, sabemos que a nossa implementação deixa ainda espaço para otimizações no que diz respeito a utilização completa de recursos disponíveis nas APIs de manipulação de ontologias. Portanto acreditamos que ainda há espaço para melhorias na questão tempo de execução.

Em resumo, mostramos através de experimentos controlados que é possível que existam ontologias onde a construção desses seja computacionalmente mais eficiente que a construção de conjuntos minimais e comprovamos que esses casos podem também ser encontrados em ontologias reais.

A construção do conjunto kernel inteiro dá ao usuário maior controle do resultado da depuração, porém acreditamos que a construção guiada do conjunto resíduo mostrada na Seção 3.4 resulta em um conjunto gerado mais próximo do esperado em uma depuração de granulação fina e uma vez que temos esse resultado, podemos utilizar a ontologia livre da inferência indesejada.

Um contratempo encontrado na depuração de granularidade fina, é que sempre se faz necessária a construção do conjunto kernel inteiro para que a depuração possa começar de fato. Otimizações podem ainda ser feitas nas funções de incisão para obter um resultado mais automático, similar a construção guiada do conjunto resíduo. Porém, essa abordagem ainda necessita da construção do conjunto kernel inteiro.

Além da depuração, propomos aqui novas formas de estratificação a serem utilizadas em conjunto com a construção de conjuntos resíduos baseadas em formas propostas para a utilização em conjunto com o kernel. Essas formas de estratificação podem ser utilizadas individualmente ou em conjunto para construir funções de seleção para os conjuntos resíduos construídos. Discutimos aqui também largamente sobre a utilização de estratégias de enfraquecimento durante a construção de um reparo para uma inferência indesejada. Mostramos que os problemas trazidos por axiomas muito complexos podem também ocorrer após a fusão, tornando difícil o descarte mínimo de informação para resolução de um conflito.

Por fim, mostramos como nossas construções foram implementadas na forma de um framework 
e que, além de implementações para a construção de elementos do conjunto resíduo e kernel, implementamos também funções para a construção dos conjuntos resíduo e kernel inteiros.

\subsection{Trabalhos Futuros}

Acreditamos que esse trabalho pode se desenvolver em algumas direções:

1. Aumento do tamanho do conjunto de dados de testes com mais ontologias reais;

2. Otimizações nos algoritmos;

3. Estudo de formas de modularização de ontologias para diminuir ainda mais o conjunto de dados manipulados;

4. Verificar dados atuais do BioPortal para checar a evolução das ontologias.

5. Implementação de plugin para editores de ontologias OWL que realizem o reparo de inferências indesejadas utilizando conjuntos resíduos;

6. Estudar a possibilidade de implementar uma versão dos algoritmos que roda de forma paralela para tirar proveito de arquiteturas multi-núcleos; 


\section{Apêndice A}

\section{Ferramentas para Fusão de Ontologias}

Durante nossa pesquisa, levantamos um conjunto de ferramentas que tinham como objetivo promover o reúso de partes de ontologias. A escolha por essas ferramentas seguiu os critérios: compatibilidade com editores mais utilizados no mercado, citação em artigos científicos, licença de software. A nossa análise levou em conta dois tipos de software, os que se propõem a realizar a fusão de fato das ontologias, e os que se propõem a realizar a depuração e reparo de ontologias.

\section{A.1 Depuração de Ontologias}

Software classificados nessa categoria auxiliam o usuário a entender a origem ou reparar inferências indesejadas, quer sejam conflitos como inconsistências e incoerências ou inferências normais para as quais os usuários desejam saber o motivo.

Avaliamos também esses software com o objetivo de estabelecer possíveis comparativos com a abordagem que propomos nesse trabalho, a de construir ontologias já reparadas a partir de uma ontologia conflitante. Portanto, as características que levantamos estavam basicamente centradas na possibilidade de calcular justificativas para qualquer tipo de inferências, facilidade de utilização através de APIs de programação e documentação.

\section{A.1.1 DION}

DION é uma ferramenta desenvolvida pelo projeto $\mathrm{SeKT}^{1}$ e possui a capacidade de construir conjuntos kernel para ontologias incoerentes. O algoritmo implementado pela ferramenta utiliza uma estratégia do tipo Black-box onde a fase de expansão utiliza uma função que seleciona um subconjunto da ontologia, no qual existe um conceito incoerente ou é inconsistente. Em seguida, o algoritmo utiliza uma estratégia comum de encolhimento com o objetivo de garantir minimalidade.

A arquitetura da ferramenta é baseada no paradigma cliente-servidor, onde foi desenvolvida uma interface web, que se comunica com o DION server. Esse por sua vez se comunica com um repositório de ontologias e com um conjunto de funções de seleções (utilizadas durante a fase expansão do Black-box). Por fim, a ferramenta implementa um cliente com interface $\mathrm{DIG}^{2}$ para a comunicação com o mecanismo de inferência.

\footnotetext{
${ }^{1}$ http://www.sekt-project.com/

${ }^{2}$ http://dl.kr.org/dig/interface.html
} 


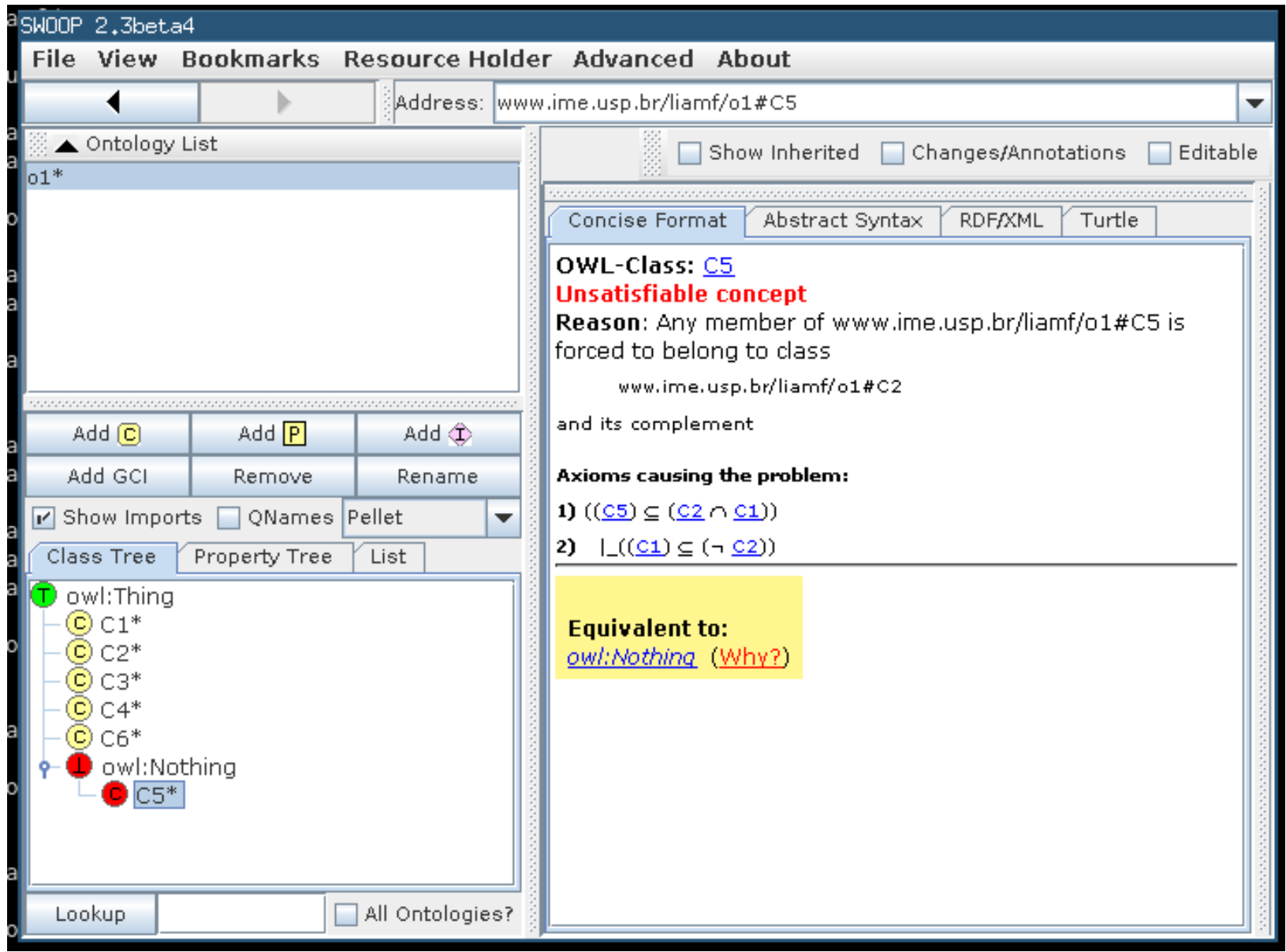

Figura A.1: Interface da ferramenta SWOOP para cálculo do conjunto Kernel

Infelizmente o projeto não recebeu novos desenvolvedores ou novas funcionalidades desde 2006. Foi implementado em Prolog, o que dificulta a integração com APIs modernas para a manipulação de ontologias, como o Jena ou OWLAPI. Por esse motivo não foram realizados muitos testes com a ferramenta.

\section{A.1.2 SWOOP}

A ferramenta $\mathrm{SWOOP}^{3}$ foi desenvolvida pelo grupo MindSwap na universidade de Maryland. É um editor de propósito geral para ontologias, com capacidades de depuração de incoerências. Sua última versão disponível, a 2.3 data de 2007.

A depuração de incoerências utiliza diretamente as ideias do serviço de depuração proposto por Kalyanpur em [Kal06]. Utiliza uma estratégia Black-box com janelas deslizantes para otimização da fase de expansão. A Figura A.1 mostra a interface da ferramenta após o cálculo do kernel para a ontologia $\mathcal{O}=\left\{C_{1} \sqsubseteq \neg C_{2}, C_{5} \sqsubseteq C_{1} \sqcap C_{2}\right\}$ :

Uma funcionalidade interessante implementada na ferramenta é a capacidade de explicar uma incoerência, utilizando linguagem natural. Na Figura A.1, no painel superior direito da figura é possível ler: "Unsatisfiable concept Reason: Any member of www.ime.usp.br/liamf/o1\#C5 is forced to belong to class www.ime.usp.br/liamf/o1\#C2 and its complement", que é o motivo da incoerência nessa ontologia.

\footnotetext{
${ }^{3}$ https://code.google.com/p/swoop/
} 


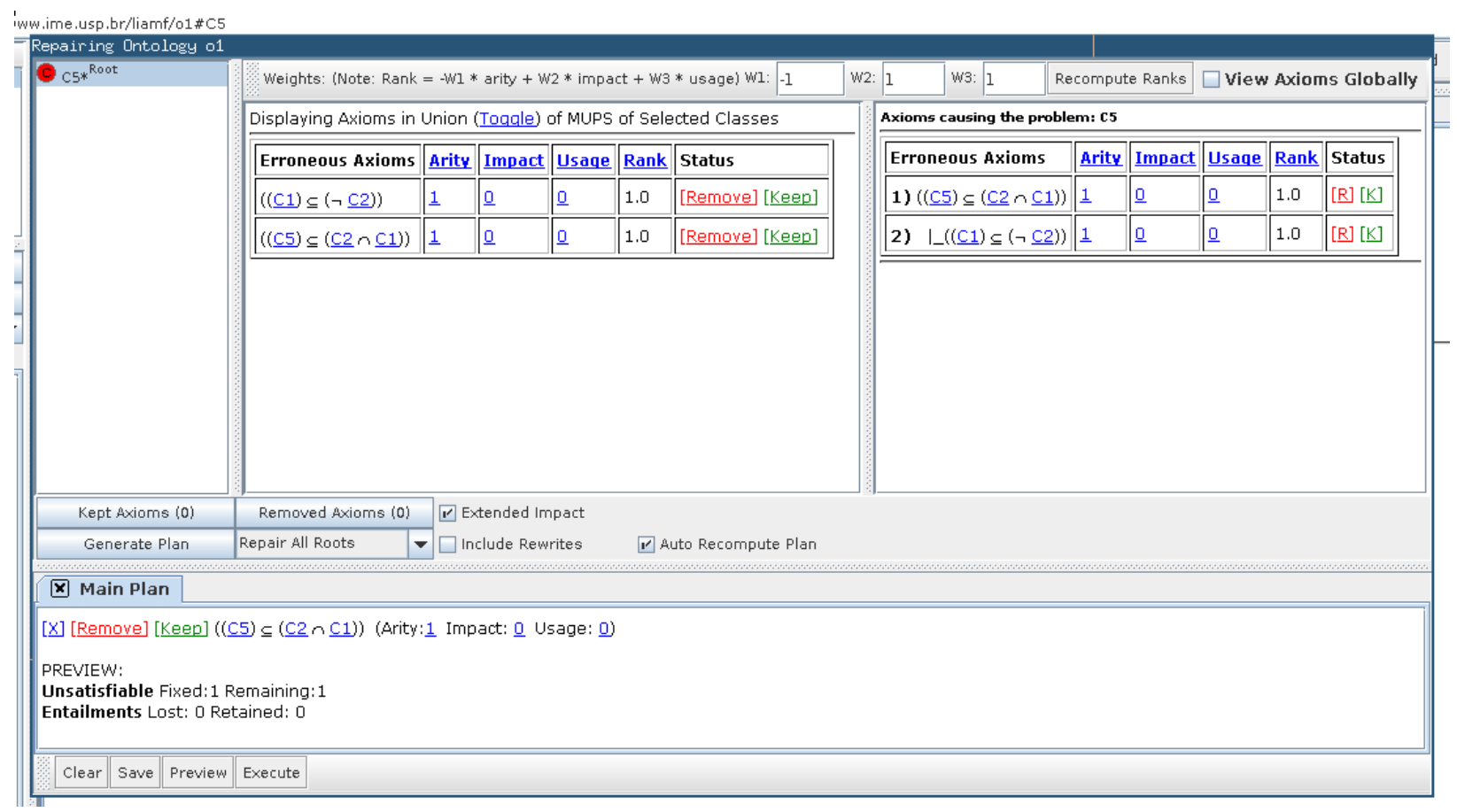

Figura A.2: Interface para construção de reparos para incoerências

A ferramenta também tem a capacidade de sugerir reparos para conflitos. O reparo é sugerido de acordo com uma nota atribuída a remoção de um dado axioma de dentro do kernel. Esse cálculo leva em conta o nível de relevância sintática, a frequência dos conceitos contidos no axioma e a relevância semântica do axioma sendo removido. A partir dessas métricas, o usuário pode gerar um plano de reparo para a ontologia, selecionando quais os axiomas que ele deseja manter e remover. A interface para essa funcionalidade pode ser vista na Figura A.2.

Na Figura A.2, no painel superior esquerdo temos a lista dos conceitos incoerentes, no painel central, o conjunto kernel contendo as justificativas para a incoerência. No painel a direita encontrase a lista de incoerências globais (todas as causas de incoerências na ontologia). No painel inferior encontra-se o plano de reparo definido pelo usuário. Também nesse espaço, o SWOOP mostra a quantidade de $\alpha$-kernels que são resolvidos com o plano de reparo e também a quantidade de inferências que são invalidadas com o plano construído.

A ferramenta apresenta funcionalidades interessantes como a capacidade de dar pesos a priorização de axiomas, e.g., o usuário pode definir que $70 \%$ da nota do reparo será dada ao número de inferências perdidas, $10 \%$ a relevância sintática e $20 \%$ a frequência de ocorrência dos conceitos no axioma a ser removido. A geração automática do plano de reparo então utiliza a maior nota como sugestão de remoção.

A adoção de um serviço de reparo automatizado pelo SWOOP se assemelha bastante ao cálculo de elementos do conjunto resíduo, que estamos realizando no nosso processo, no propósito de sugerir reparos para o usuário. Entretanto, o cálculo automatizado de reparos resulta em maior complexidade após o cálculo do conjunto kernel. Acreditamos que essa complexidade pode ser evitada utilizando a construção direta do reparo (elemento do conjunto resíduo).

Infelizmente, a ferramenta não trabalha com a depuração de ontologias inconsistentes e não possui a capacidade de depurar inferências gerais, embora possua a capacidade de calcular justificativas para as mesmas, como pode ser visto na Figura A.3. 


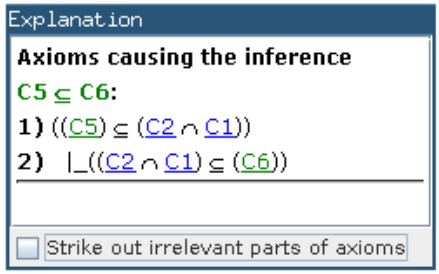

Figura A.3: Interface para cálculo de justificativas para inferências

O código da ferramenta se encontra disponível utilizando uma licença de software livre. Infelizmente, o código não faz uso de bibliotecas atuais para a manipulação de ontologias, tornando a tarefa de reutilizar sua implementação em programas mais atuais difícil.

\section{A.1.3 Neon Toolkit + RADON Plugin}

Para lidar com o problema da depuração de ontologias inconsistentes/incoerentes, a suíte de desenvolvimento Neon Toolkit ${ }^{4}$ possui um plugin chamado RaDON ${ }^{5}$. Esse plugin implementa a capacidade de cálculo dos chamados conjuntos MIS - Minimal Inconsistent Subset e MIPS Minimal Incoherence-Preserving Sub-ontology.

De fato, esses conjuntos correspondem a conjuntos kernel, calculados para $T \sqsubseteq \perp$ e $C \sqsubseteq \perp$, para um $C$ qualquer na ontologia. Por essa definição, o plugin não é capaz de encontrar justificativas para inferências não triviais que não envolvem inconsistências ou incoerências. A interface do plugin pode ser vista na Figura A.4.

Na Figura A.4, é possível ver que as formas de reparos para a ontologia estão divididas em Reparo de Inconsistência e Incoerência. Nesse exemplo mostrado, modelamos a ontologia $\mathcal{O}=$ $\{$ Class $2 \sqsubseteq$ Class 1, Class $4 \sqsubseteq$ Class1,Class $2 \sqsubseteq$ Class 3, Class $4 \sqsubseteq$ Class 3, Class $1 \sqsubseteq \neg$ Class 3$\}$. Dessa ontologia, vemos que $\mathcal{O} \models\{$ Class $2 \sqsubseteq \perp$, Class $4 \sqsubseteq \perp\}$. A ferramenta apresenta então as justificativas para essas inferências como visto na Figura A.5.

Ao usuário são apresentadas opções de reparo manual, onde o usuário escolhe o axioma que será removido para resolver a inconsistência ou incoerência; ou a opção automatizada, onde o sistema escolhe o axioma que mais se repete em todos os elementos do conjunto kernel, ou seja, o critério da frequência apresentado em [Kal06]. Nesse caso, o sistema calcula uma "nota" para a remoção de um determinado axioma, sendo essa nota o valor de elementos do conjunto kernel no qual o axioma aparece. A apresentação da nota ao usuário no reparo automático é vista na Figura A.6.

A ferramenta é disponibilizada sob licença Eclipse Public License e o código está disponível para análise. Após análise, descobrimos que a ferramenta utiliza algumas heurísticas para melhorar o desempenho tanto do algoritmo Black-box quanto para o cálculo da árvore de cortes mínimos. As funcionalidades disponíveis na ferramenta estão listada em $\left[\mathrm{JHQ}^{+} 09\right]$.

Para o cálculo da árvore de cortes mínimos a ferramenta utiliza a terminação precoce de caminhos e a reutilização de nós. Já para o algoritmo Black-box para encontrar um único elemento do conjunto kernel são utilizadas a expansão trivial, com a adição de todos os elementos em um conjunto que é passado para a fase de encolhimento. E o encolhimento é feito via técnica chamada fast prunning, baseada na ideia das janelas deslizantes, para a remoção de mais de um axioma por

\footnotetext{
${ }^{4}$ http://neon-toolkit.org/

${ }^{5}$ http://neon-toolkit.org/wiki/2.3.1/RaDON
} 


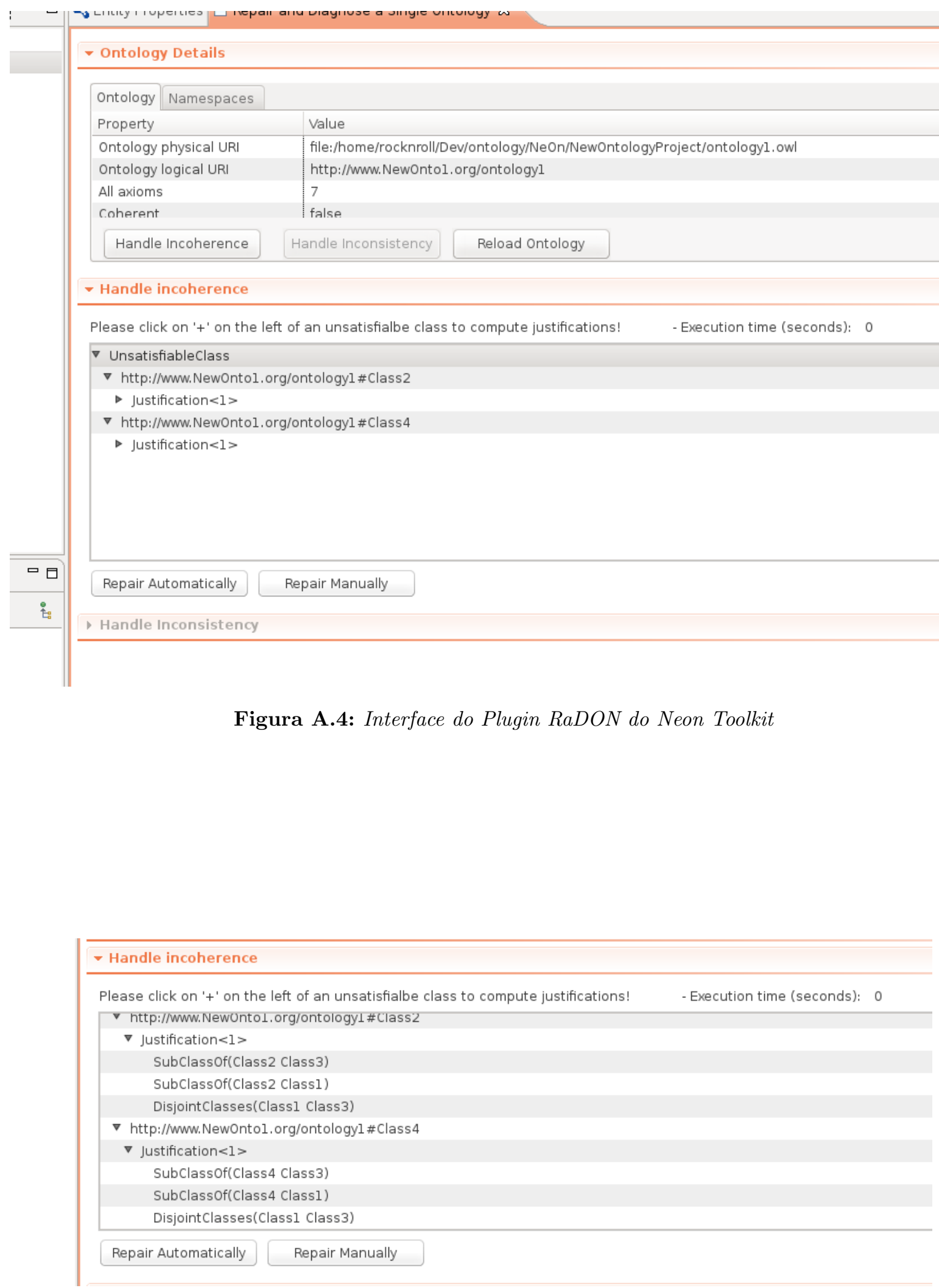

Figura A.5: Cálculo de Justificativas no RaDON 


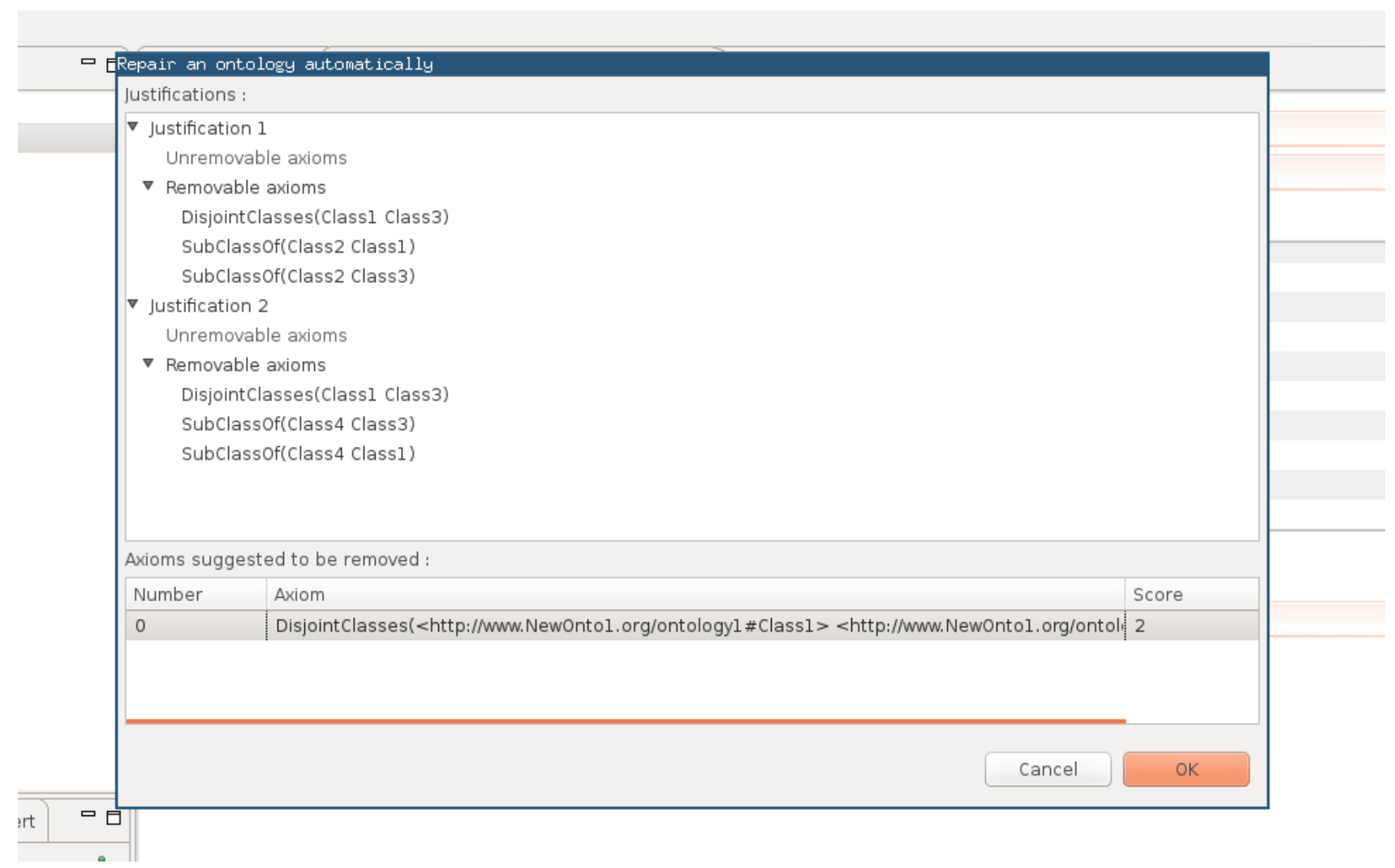

Figura A.6: Cálculo da nota do Reparo de uma incoerência

vez.

A ferramenta apresenta funcionalidades interessantes para a depuração de erros lógicos em ontologias. Infelizmente não pode ser utilizada para a construção de justificativas para inferências que não estejam envolvidas em problemas lógicos.

A ideia de sugerir estratégias de reparo automáticas é interessante e se aproxima do que estamos propondo com a nossa abordagem. No entanto, a ferramenta não permite que o usuário defina uma estratégia (automática) para escolher quais axiomas serão removidos para construir um reparo, i.e., não existem estratégias de estratificação alternativas e por consequência escolhas de funções de incisão.

\section{A.1.4 Protégé + OWLExplanation Plugin}

O Protégé é um dos editores mais utilizados para a construção de ontologias. Foi desenvolvido utilizando uma arquitetura modular que possibilita a inclusão de plugins, assim como o Neon Toolkit. A ferramenta clamada OWLExplanation Plugin ${ }^{6}$ foi desenvolvida com o objetivo de calcular explicações ou justificativas para qualquer inferência realizada pelo Protégé, inclusive incoerências.

A Figura A.7 mostra uma ontologia incoerente. O botão apontado pela seta é utilizado para cálculo de justificativas. Nesse caso, o Protégé está indicando que um axioma possui, como super classe, a classe especial Nothing, que representa $\perp$. A interface do cálculo de justificativa para incoerências pode ser vista na Figura A.7 e para inconsistências na Figura A.8.

A ferramenta tem a capacidade de construir todas as justificativas para uma dada inferência, i.e., o conjunto kernel completo, mostrando a quantidade de $\alpha$-kernels nos quais cada axioma se

\footnotetext{
${ }^{6}$ https://github.com/matthewhorridge/owlexplanation
} 


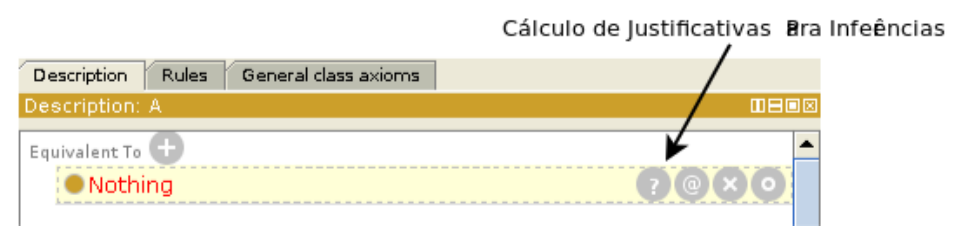

Figura A.7: Interface da ferramenta OWLExplanation - Justificativa para Incoerência

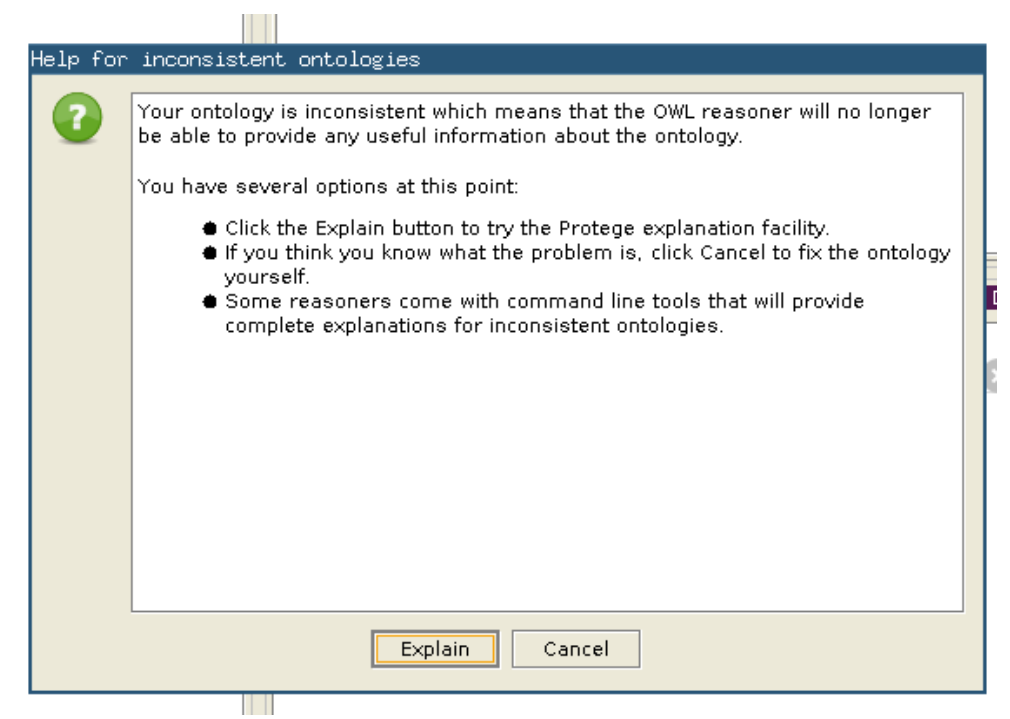

Figura A.8: Interface da ferramenta OWLExplanation - Justificativa para Inconsistência

encontra. A Figura A.9 mostra a utilização da ferramenta para o cálculo de justificativas para a ontologia apresentada no exemplo A.1.

Exemplo A.1 . Suponha a ontologia:

$$
\begin{aligned}
\mathcal{O}=\{ & C_{1} \sqsubseteq \neg C_{2}, \\
& C_{3} \sqsubseteq C_{1}, \\
& C_{3} \sqsubseteq C_{2}, \\
& C_{4} \sqsubseteq C_{1} \sqcap C_{2}, \\
& \left.\left\{I_{3}\right\} \sqsubseteq C_{3} \sqcap C_{4} \sqcap C_{5}\right\} \models \top \sqsubseteq \perp
\end{aligned}
$$

Se considerarmos $\alpha=\top \sqsubseteq \perp$ e calcularmos o conjunto kernel $\mathcal{K}$ para $\mathcal{O}$ obtemos:

$$
\begin{aligned}
& \mathcal{O} \Perp(\top \sqsubseteq \perp)=\left\{C_{4} \sqsubseteq C_{1} \sqcap C_{2}, C_{1} \sqsubseteq \neg C_{2},\left\{I_{3}\right\} \sqsubseteq C_{3} \sqcap C_{4} \sqcap C_{5}\right\}, \\
&\left.\left\{C_{3} \sqsubseteq C_{1}, C_{1} \sqsubseteq \neg C_{2},\left\{I_{3}\right\} \sqsubseteq C_{3} \sqcap C_{4} \sqcap C_{5}, C_{3} \sqsubseteq C_{2}\right\}\right\}
\end{aligned}
$$

Na Figura A.9, podemos ver a informação de que os axiomas $C_{1} \sqsubseteq \neg C_{2}$ e $\left\{I_{3}\right\} \sqsubseteq C_{3} \sqcap C_{4} \sqcap C_{5}$ estão presentes em todos os $\alpha$-kernel. Assim como no caso do RaDON, essa informação auxilia no processo de remoção, quando estamos reparando incoerências ou inconsistências. Porém, ao contrário do RaDON, essa ferramenta não sugere formas de reparos para inconsistências ou incoerências.

Uma característica interessante do OWLExplanation plugin é que ele tem a capacidade de construir justificativas de granularidade fina, utilizando o algoritmo (função $\delta$ ) proposto por Horridge 


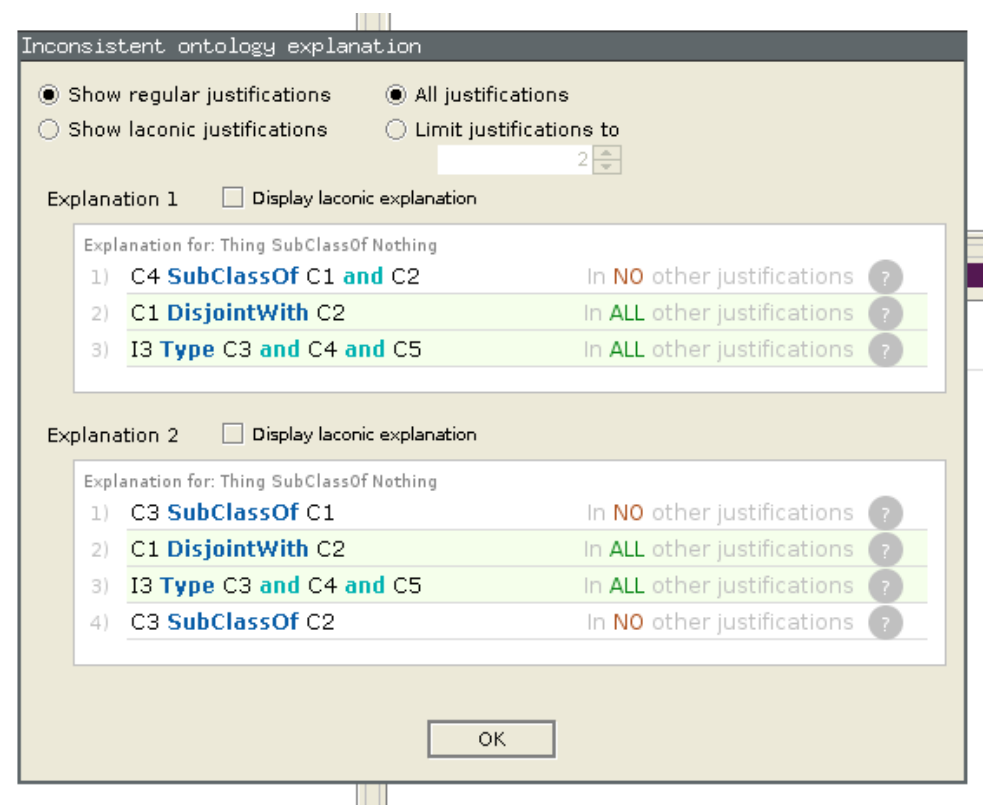

Figura A.9: OWLExplanation - Cálculo de Justificativas

em [Hor11]. Essa forma de cálculo de justificativa é chamada de explicação lacônica. O resultado do cálculo das explicações lacônicas para o kernel apresentado na Figura A.9 pode ser visto na Figura A.10. É possível ver que todos os axiomas são quebrados em versões enfraquecidas.

O programa mostra duas possibilidades de enfraquecimento (explicações lacônicas). Na Figura A.10, o cálculo de justificativas foi feito em uma base que foi enfraquecida. Por conta de fenômenos de mascaramento, vistos no Capítulo 3, o número de justificativas para a inconsistência na ontologia é maior, caso essa tenha seus axiomas enfraquecidos. O Exemplo A.2 mostra a diferença entre o enfraquecimento de justificativas e o cálculo de justificativas em uma base enfraquecida.

Exemplo A.2 Suponha a ontologia $\mathcal{O}$ apresentada no Exemplo A.1. Se utilizarmos a função $\delta$ proposta por Horridge em [Hor11], obtemos a base enfraquecida $\mathcal{O}^{\prime}$ :

$$
\begin{aligned}
\delta(\mathcal{O})=\{ & C_{1} \sqsubseteq \neg C_{2}, \\
& C_{3} \sqsubseteq C_{1}, \\
& C_{3} \sqsubseteq C_{2}, \\
& C_{4} \sqsubseteq C_{1}, \\
& C_{4} \sqsubseteq C_{2}, \\
& \left\{I_{3}\right\} \sqsubseteq C_{3}, \\
& \left\{I_{3}\right\} \sqsubseteq C_{4}, \\
& \left.\left\{I_{3}\right\} \sqsubseteq C_{5}\right\} \models \top \sqsubseteq \perp
\end{aligned}
$$

Se calcularmos o kernel, considerando novamente $\alpha=\top \sqsubseteq \perp$, mas dessa vez para $\mathcal{O}^{\prime}$ obtemos 4 


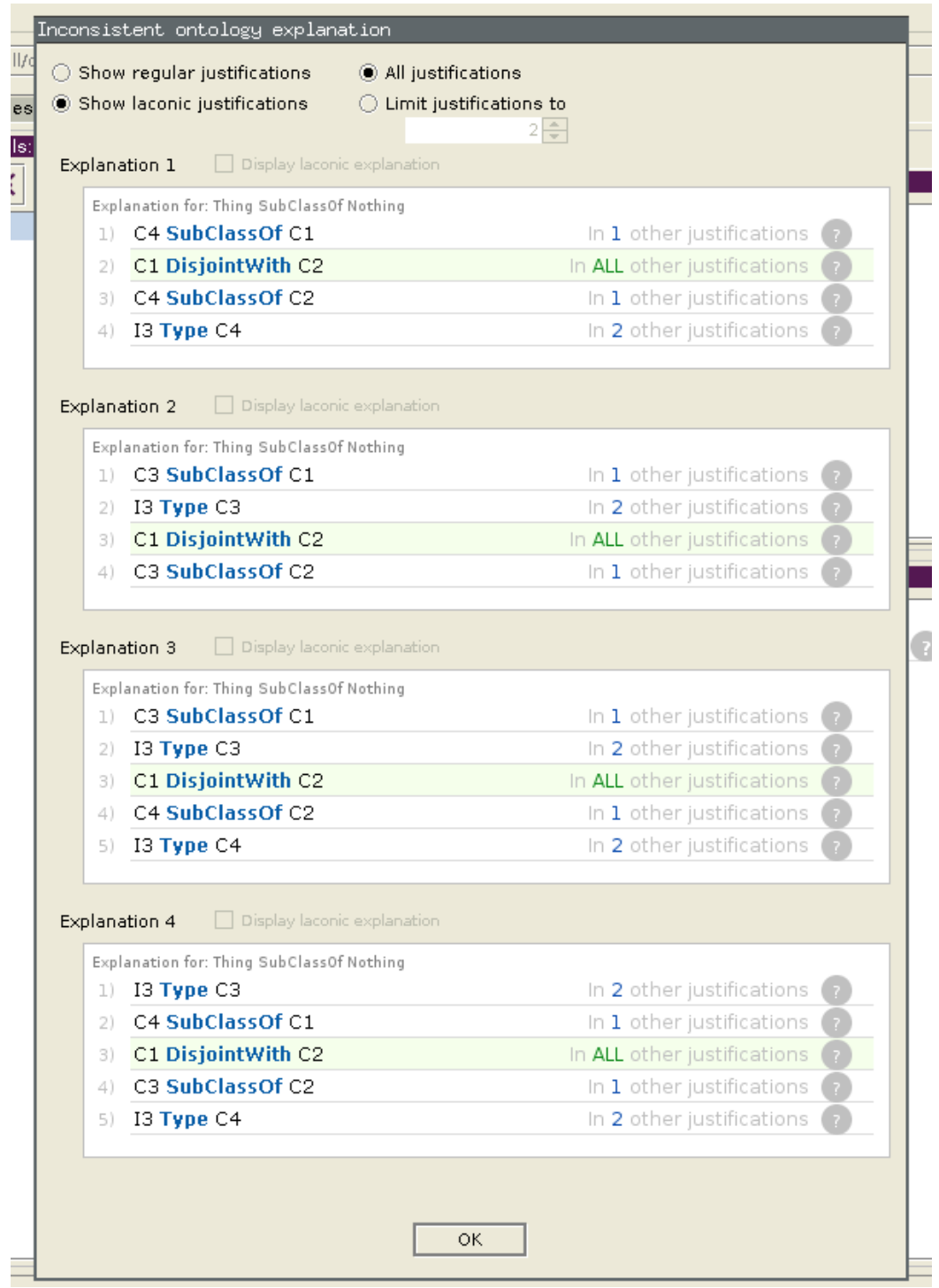

Figura A.10: $O W L$-explanation - Cálculo de Justificativas Lacônicas 
$\alpha$-kernel:

$$
\begin{aligned}
\mathcal{O}^{\prime} \Perp(\top \sqsubseteq \perp)=\{ & \left\{C_{4} \sqsubseteq C_{1}, C_{1} \sqsubseteq \neg C_{2}, C_{4} \sqsubseteq C_{2},\left\{I_{3}\right\} \sqsubseteq C_{4}\right\} \\
& \left\{C_{3} \sqsubseteq C_{1},\left\{I_{3}\right\} \sqsubseteq C_{3}, C_{1} \sqsubseteq \neg C_{2}, C_{3} \sqsubseteq C_{2}\right\} \\
& \left\{C_{3} \sqsubseteq C_{1},\left\{I_{3}\right\} \sqsubseteq C_{3}, C_{1} \sqsubseteq \neg C_{2}, C_{4} \sqsubseteq C_{2},\left\{I_{3}\right\} \sqsubseteq C_{4}\right\} \\
& \left.\left\{\left\{I_{3}\right\} \sqsubseteq C_{3}, C_{3} \sqsubseteq C_{2}, C_{1} \sqsubseteq \neg C_{2}, C_{4} \sqsubseteq C_{1},\left\{I_{3}\right\} \sqsubseteq C_{4}\right\}\right\}
\end{aligned}
$$

O mesmo resultado visto na Figura A.10. Por outro lado, se tivéssemos aplicado a função $\delta$ ao conjunto kernel do Exemplo A.1, obteríamos o conjunto incoerente de axiomas $\mathcal{B}$ :

$$
\begin{aligned}
\delta(\mathcal{O} \Perp(\top \sqsubseteq \perp))=\{ & \left\{C_{4} \sqsubseteq C_{1}, C_{4} \sqsubseteq C_{2}, C_{1} \sqsubseteq \neg C_{2},\left\{I_{3}\right\} \sqsubseteq C_{4},\left\{I_{3}\right\} \sqsubseteq C_{3},\left\{I_{3}\right\} \sqsubseteq C_{5}\right\} \\
& \left.\left\{C_{3} \sqsubseteq C_{1}, C_{3} \sqsubseteq C_{2}, C_{1} \sqsubseteq \neg C_{2},\left\{I_{3}\right\} \sqsubseteq C_{3},\left\{I_{3}\right\} \sqsubseteq C_{4},\left\{I_{3}\right\} \sqsubseteq C_{5}\right\}\right\}
\end{aligned}
$$

É importante notar que o conjunto $\mathcal{B}$ não é mais minimal com relação a inclusão. Temos que descartar os axiomas que não fazem parte do conflito: $\left\{I_{3}\right\} \sqsubseteq C_{3},\left\{I_{3}\right\} \sqsubseteq C_{5}$ para $\mathcal{B}_{1} e\left\{I_{3}\right\} \sqsubseteq$ $C_{4},\left\{I_{3}\right\} \sqsubseteq C_{5}$ para $\mathcal{B}_{2}$, resultando no novo conjunto kernel enfraquecido $\mathcal{K}^{\prime}$ :

$$
\begin{aligned}
\mathcal{K}^{\prime}=\{ & \left\{C_{4} \sqsubseteq C_{1}, C_{4} \sqsubseteq C_{2}, C_{1} \sqsubseteq \neg C_{2},\left\{I_{3}\right\} \sqsubseteq C_{4}\right\} \\
& \left.\left\{C_{3} \sqsubseteq C_{1}, C_{3} \sqsubseteq C_{2}, C_{1} \sqsubseteq \neg C_{2},\left\{I_{3}\right\} \sqsubseteq C_{3}\right\}\right\}
\end{aligned}
$$

O cálculo do kernel enfraquecido ajuda o projetista a ver quais partes dos axiomas não fazem parte da inferência. Entretanto, o cálculo não é de muita ajuda quando temos de atualizar a base de conhecimento com o resultado de uma função de incisão, pois a base ainda conterá os axiomas de granularidade grossa. É preciso manter a referência de quais axiomas estavam presentes em $\alpha$ kernels e após o enfraquecimento, precisamos realizar a contração da ontologia por esses axiomas, seguida da expansão pelo resultado da função de incisão mais a informação que foi descartada do $\alpha$-kernel pós-enfraquecimento, i.e., retiramos os axiomas de granularidade grossa, enfraquecemos o kernel, mantendo a lista de axiomas que não fazem parte do kernel (partes de axiomas irrelevantes), em seguida executamos a função de incisão, e expandimos a ontologia pelo resultado da incisão mais o que foi recém descartado do kernel. O Exemplo A.3 mostra como essa ideia funciona a partir do exemplo A.1.

Exemplo A.3 Considerando o conjunto kernel enfraquecido $\mathcal{K}^{\prime}$ do Exemplo A.2, descartamos o seguinte conjunto de informações $\mathcal{X}$ :

$$
\mathcal{X}=\left\{\left\{I_{3}\right\} \sqsubseteq C_{3} \sqcap C_{5},\left\{I_{3}\right\} \sqsubseteq C_{4} \sqcap C_{5}\right\}
$$

Suponhamos que tenha sido utilizada uma função de incisão que removesse do kernel enfraquecido os axiomas: $\left\{I_{3}\right\} \sqsubseteq C_{3}$ e $\left\{I_{3}\right\} \sqsubseteq C_{4}$, a operação de resolução de conflitos teria que realizar a contração da ontologia $\mathcal{O}$ por $\left\{I_{3}\right\} \sqsubseteq C_{3} \sqcap C_{4} \sqcap C_{5}$ e realizar a expansão por $\left\{I_{3}\right\} \sqsubseteq C_{5}$.

Como visto, a utilização do enfraquecimento para a depuração de ontologias deve ser feita de forma cuidadosa para evitar que informações relevantes para a ontologia e não para o conflito sejam removidas. Nesse caso, o enfraquecimento do conjunto kernel no lugar da ontologia inteira resulta 
em um ganho de desempenho, por não termos que analisar os axiomas um a um aplicando a função $\delta$.

A implementação do OWLExplanation Plugin utiliza as estratégias de otimização tanto para o algoritmo de Reiter quanto para o Black-Box. De acordo com o que foi descrito por Horridge em [Hor11], a implementação utiliza otimizações de reutilização de nós e término precoce de caminhos para o algoritmo para construção da árvore de cortes mínimos. Para o algoritmo do Black-box, o autor afirma que a implementação utiliza a divisão e conquista para a fase de encolhimento.

Selecionamos essa ferramenta para comparar com a abordagem que propomos nesse trabalho para cálculo de conjuntos resíduo por possuir a capacidade de calcular justificativas para inferências que não sejam somente inconsistências ou incoerências.

\section{A.2 Ferramentas para Reuso de Ontologias}

Nesta Seção faremos uma breve revisão de algumas ferramentas utilizadas para promover o reúso de ontologias. A primeira delas, chamada PROMPT é a referência mais clássica para ferramenta que se propõe a realizar a fusão de ontologias. Em seguida listamos algumas outras ferramentas mais modernas que possuem o propósito de prover a capacidade de reutilizar ontologias ou trechos de ontologias.

\section{A.2.1 PROMPT}

A primeira ferramenta analisada foi o PROMPT. A ferramenta é distribuída junto da suíte PROMPT para merging e comparação de ontologias, é a mais referenciada por artigos sobre fusão e reúso de ontologias. É distribuída sob a forma de complemento para o Protégé 3.0/3.1, portanto é desenvolvida em Java e sua primeira versão data de 2000 [NM00]. A suíte evoluiu para a sua versão atual, a $3.0^{7}$, de 2006, de onde as atividades de casamento e fusão de ontologias, que são executadas, respectivamente, pelas ferramentas Anchor-Prompt [NM01] e iPrompt [NM03], estão bem definidas.

O funcionamento do PROMPT está dividido em atividades, que basicamente fazem sugestões ao usuário sobre um conjunto de operações pré-definidas na ferramenta. Inicialmente a ferramenta sugere operações a serem executadas, o usuário então seleciona manualmente quais gostaria de executar, a ferramenta as executa, em seguida verifica a consistência da ontologia de destino e caso essa se encontre inconsistente, ele sugere novas operações. O processo passa por várias iterações até que o sistema não recomende nenhuma operação e a ontologia de destino se encontre no estado consistente. O conjunto de operações presentes no PROMPT é composto de:

- Unir duas classes $A$ e $B$ (merge-classes operation):

- Criar nova classe $M$ na ontologia de destino $O_{m}$;

- Para cada classe $C$ que seja Super ou Subclasse de $A$ ou $B$ :

* Se existe uma classe $C$, já copiada em $O_{m}$, adicionar $C$ como super ou subclasse de $M$;

- Para cada slot $S$ conectado a $A$ ou $B$ :

\footnotetext{
${ }^{7}$ http://protegewiki.stanford.edu/wiki/PROMPT
} 
* Se não existe uma imagem $S_{i}$ de $S$ em $O_{m}$, copiar $S$ para $O_{m}$;

- Para cada imagem $S_{i}$ de $S$ em $O_{m}$, conectar $S_{i}$ a $M$;

- Unir duas propriedades $S_{1}$ e $S_{2}$ (merge-slots operation):

- Criar nova propriedade $S_{m}$ na ontologia de destino $O_{m}$;

- Para cada classe $C$ que esteja no domínio ou imagem de $S_{1}$ ou $S_{2}$ :

* Se existe uma imagem $C_{i}$ de $C$ em $O_{m}$, adicionar $C_{i}$ a imagem ou ao domínio de $S_{m}$

- Se $S_{1}$ ou $S_{2}$ já se encontrava em $O_{m}$, todas as referências prévias a $S_{1}$ ou $S_{2}$ são atualizadas a $S_{m}$ e em seguida essas propriedades são removidas;

- Unir duas instâncias ${ }^{8} I_{1}$ e $I_{2}$ (merge-instances operation):

- Criar nova instância $I_{m}$ na ontologia de destino $O_{m}$;

- Para as classes $C_{1}$ e $C_{2}$, das quais $I_{1}$ e $I_{2}$ sejam instâncias respectivamente:

* Se não existe uma cópia de $C_{1}$ nem de $C_{2}$ em $O_{m}$, copiar $C_{1}$ e $C_{2}$ para $O_{m}$ e definir uma nova classe $C$ como tipo de $I_{m}$ (operação de shallow copy);

- Se $C_{1}$ e $C_{2}$ já possuírem imagens em $O_{m}$ e essas imagens são diferentes, sugerir realizar a fusão das imagens de $C_{1}$ e $C_{2}$;

- Unir duas instâncias $I_{1}$ e $I_{2}$ (merge-instances operation):

- Para cada valor $V$ da propriedade $S$ conectada a $I_{1}$ ou $I_{2}$ :

- Se $V$ for um valor primitivo (string, número, etc.), adicionar $V$ ao valor da imagem da propriedade $S$ para $I_{m}$;

- Se $V$ é uma classe e existe uma imagem $V_{i}$ de $V$ em $O_{m}$, adicionar $V_{i}$ ao valor da imagem da propriedade $S$ para $I_{m}$;

Exemplo de Fusão com o PROMPT: Ao realizar a fusão das duas ontologias presentes na Figura A.11, o sistema faz o conjunto de sugestões apresentados na Figura A.12. O nome da operação sugerida pode ser visto na primeira coluna da lista chamada de "TO DO List". Pode-se ver que inicialmente ele sugere que cópias sejam feitas das classes Professor_Titular e Professor_Associado para a ontologia de destino. A ontologia pós fusão pode ser vista na Figura A.13. No entanto, se o usuário acredita que as classes Professor_Titular e Professor_Associado devem sofrer fusão, ele pode então, no lugar de realizar as cópias sugeridas pela ferramenta, cadastrar qual operação deve ser realizada (dentre as disponíveis). Nesse caso, ele cadastra a operação de fusão de classes, o que pode ser visto na Figura A.14.

O PROMPT é capaz de lidar com o seguinte conjunto de inconsistências:

- Conflito de nome - Figura A.15:

- Classes tem de ter nomes únicos em uma ontologia;

\footnotetext{
${ }^{8} \mathrm{O}$ modelo de conhecimento adotado pelo Prompt somente possibilita uma classe como tipo de uma dada instância;
} 

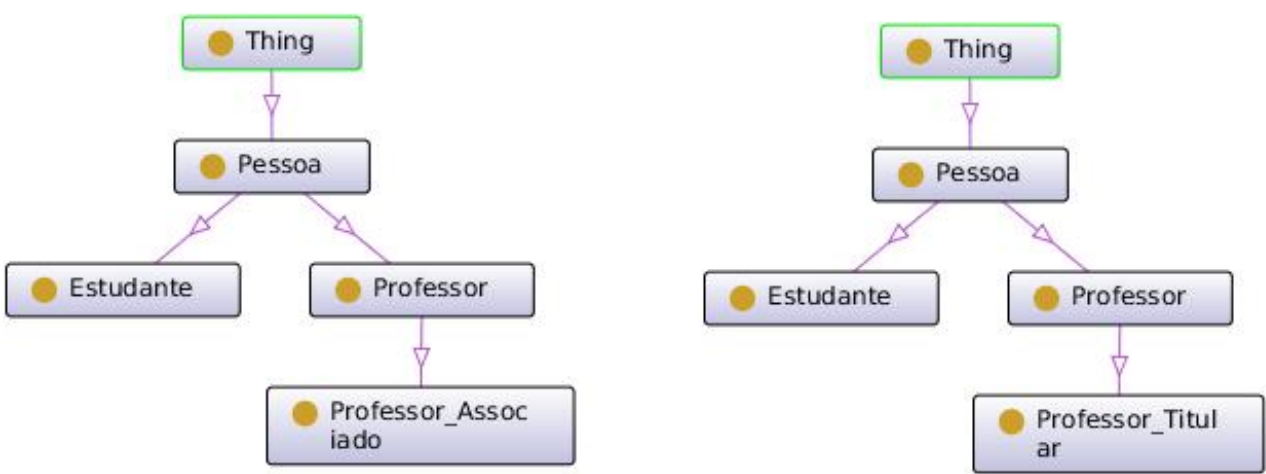

Figura A.11: Fusão no PROMPT - entradas

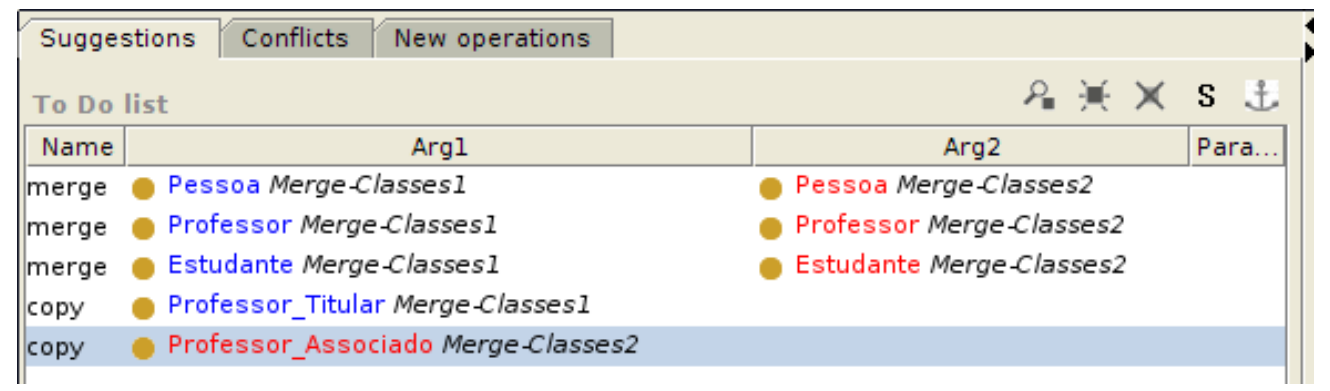

Figura A.12: Fusão no PROMPT - sugestões

\begin{tabular}{l}
\hline Result classes Result slots \\
current \\
\hline $0:$ THING \\
\hline \% $:$ SYSTEM-CLASS \\
P Pessoa \\
९ Professor \\
O Professor_Titular--Merge-Classes1 \\
Professor_Associado--Merge-Classes2 \\
Estudante
\end{tabular}

Figura A.13: Fusão no PROMPT - ontologia pós fusão

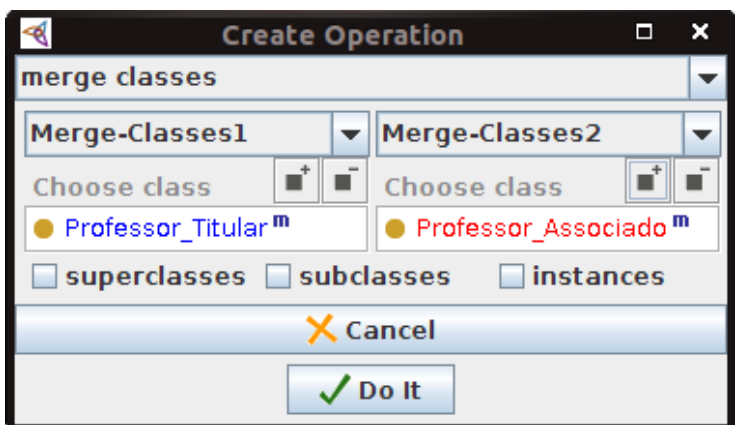

Figura A.14: Fusão no PROMPT - cadastramento de operação de fusão de classes 
- Cópias podem causar a existência de classes com mesmos nomes;

- No exemplo, se a operação de copy for realizada para a classe Pessoa de ambas as ontologias;

- Para resolver essa inconsistência, o PROMPT sugere a renomeação de uma das classes;

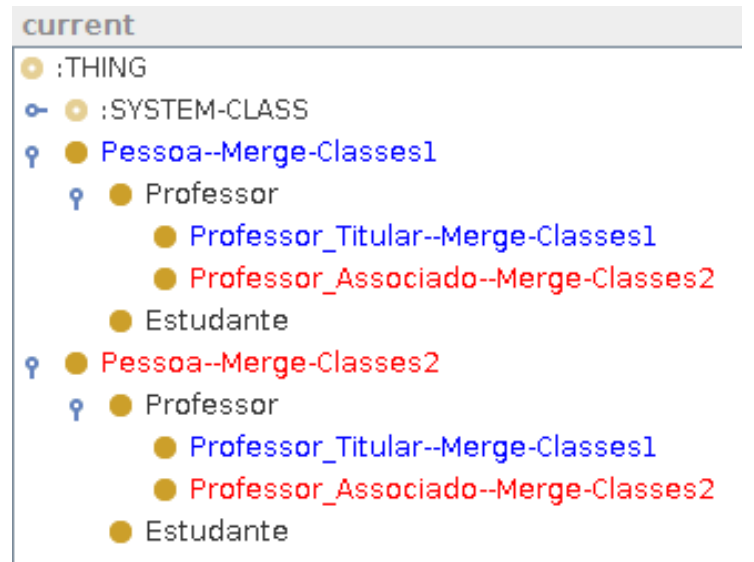

Figura A.15: Conflito de nome

- Conflito de Referências Pendentes (Dangling References) - Figura A.16:

- Classes copiadas junto com propriedades cujas imagens estão faltando;

- No exemplo Anterior, caso existisse a propriedade orienta que liga Professor a Estudante;

- Caso seja feita a cópia ou a união do conceito Professor e não a cópia de estudante;

- Para resolver esse tipo de inconsistência o PROMPT sugere que os conceitos que se encontram faltando sejam copiados para a ontologia de destino;

\begin{tabular}{|c|c|c|c|c|c|c|}
\hline Suggestions & Conflicts & New operations & 5 & Result classes & Result slots & Result i \\
\hline$\because \cdots \cdots \cdots \cdots \cdots \cdots \cdots \cdots \cdots \cdots \cdots$ & $\therefore \cdots \cdots$ & $\cdots \cdots$ & & \multicolumn{3}{|l|}{ current } \\
\hline \multicolumn{4}{|c|}{ Suggested solutions for selected conflict } & \multirow{4}{*}{\multicolumn{3}{|c|}{$\begin{array}{l}\text { :THING } \\
\text { \% :SYSTEM-CLASS } \\
\text { ९ Pessoa } \\
\text { Professor }\end{array}$}} \\
\hline Name & Arql & \begin{tabular}{r|r} 
& Arg2
\end{tabular} & Params & & & \\
\hline \multicolumn{4}{|c|}{ merge Estudante Merge-Classes1 Estudante Merge-Classes2 } & & & \\
\hline copy Estud & te Merge-C & ssesl & & & & \\
\hline
\end{tabular}

Figura A.16: Conflito de Referências Pendentes

- Redundância na hierarquia de classes - Figura A.18:

- Ocorrem quando, após o merging, existe mais que um caminho que liga uma classe a sua superclasse;

- A Figura A.17 mostra duas hierarquias a sofrerem fusão. Durante a fusão o usuário opta por realizar a operação de fusão de classes para todas as classes, com exceção de Aluno_Graduacao e Aluno_Bacharelado. Assim, a ontologia resultante terá dois caminhos para atingir a super-classe Pessoa, saindo da classe Aluno_Ciencia_da_Computacao, um passando por Aluno_Graduacao e outro por Aluno_Bacharelado; 

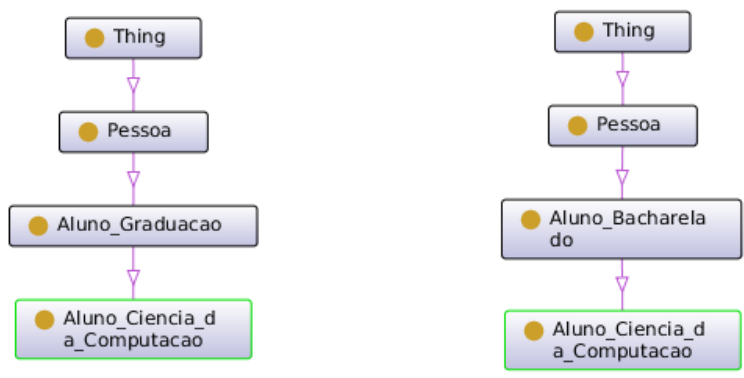

Figura A.17: Redundância na hierarquia de classes - exemplo

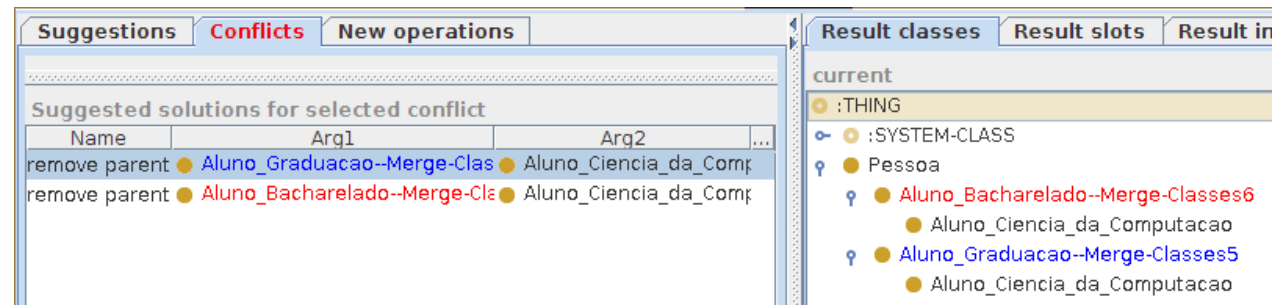

Figura A.18: Redundância na hierarquia de classes - sugestão do PROMPT

- Para resolver esse problema, o PROMPT sugere que algum dos caminhos seja removido;

- Valores de propriedades violando restrições de valores de propriedades - Figura A.19:

- Ocorrem quando, após a fusão, as propriedades violam cardinalidades ou tipos dos valores;

- Na Figura A.19 pode-se ver duas ontologias que possuem a restrição que um professor somente orienta um aluno. Ao realizar a fusão, o PROMPT realiza o casamento entre os dois indivíduos chamados Professor1. A fusão desses conceitos, junto da cópia dos indivíduos Aluno1 e Aluno2 resultariam na violação de uma restrição de cardinalidade, que diz que o Professor1 só pode orientar um único aluno;

- Para resolver esse problema, o PROMPT realiza duas cópias do conceito, acrescentando um sufixo ao nome do indivíduo, mantendo assim a consistência, como pode ser visto na Figura A.20;

- Único tipo de inconsistência tratada pelo PROMPT que pode ser uma inconsistência lógica

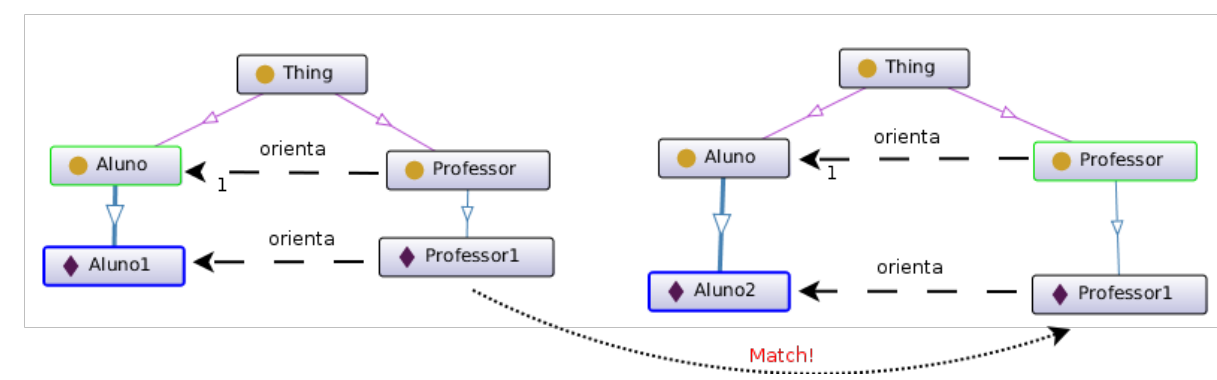

Figura A.19: Valores de propriedades violando restrições de valores de propriedades - cenário 


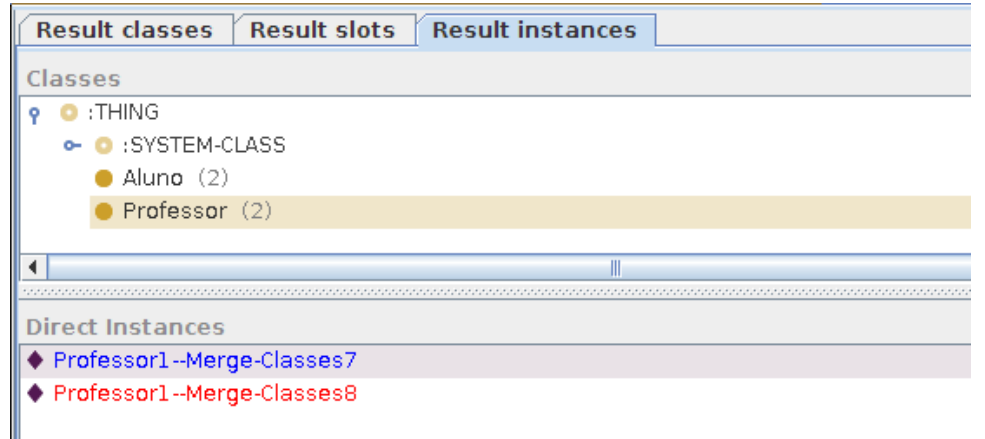

Figura A.20: Valores de propriedades violando restrições de valores de propriedades - cópias em separado de conceitos que ameaçam a consistência

O detalhado estudo da ferramenta, testando cada um dos tipos de inconsistência que são tratados pelo PROMPT, foi realizado para verificar a existência de correspondências entre os tipos de inconsistências por essa ferramenta identificados e tratados e os tipos de consistências lógicas que resultam das cláusulas de disjunção e diferença. O resultado desse estudo aponta que a ferramenta PROMPT é incapaz de lidar com inconsistências lógicas. Na verdade, essa incapacidade se dá pelo fato de que tais cláusulas de disjunção e diferença não estavam incorporadas a tecnologia para a especificação de ontologias. Então, quando o PROMPT foi concebido, não existia a preocupação com as inconsistências lógicas.

\section{A.2.2 Protégé Merge Ontologies Refactor}

Desde o lançamento da versão 4 do Protégé, o mesmo acompanha uma funcionalidade para realizar a fusão de ontologias. É preciso para isso abrir as ontologias, que se deseja unir, no mesmo workspace e ativar a funcionalidade. A Figura A.21 mostra a tela de seleção de ontologias para fusão.

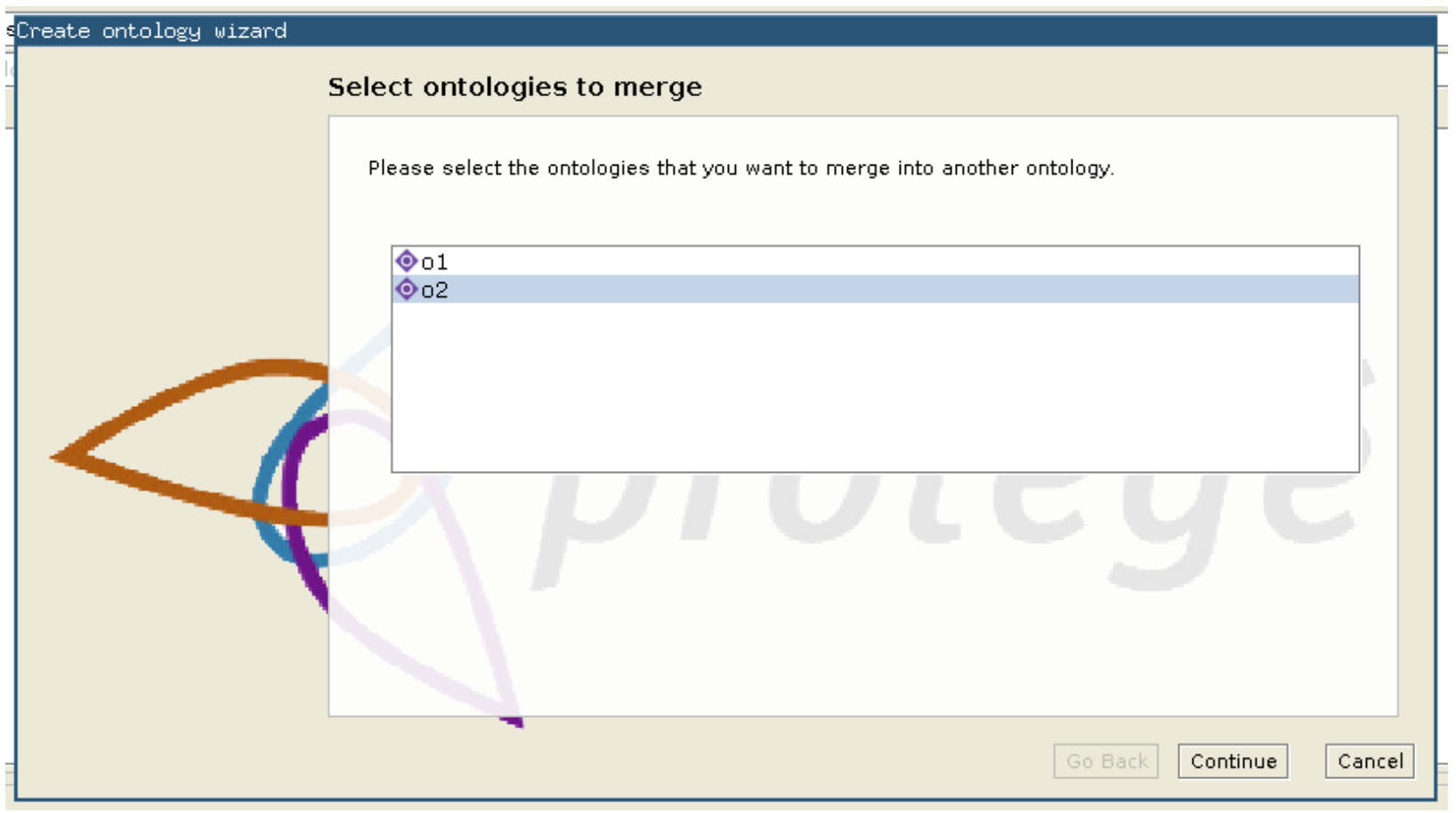

Figura A.21: Seleção de Ontologias para realizar Fusão 
A ferramenta impõe uma outra restrição que é a de que, além das ontologias estarem no mesmo workspace, essas não podem possuir o mesmo identificador universal IRI, como pode ser visto na Figura A.22.

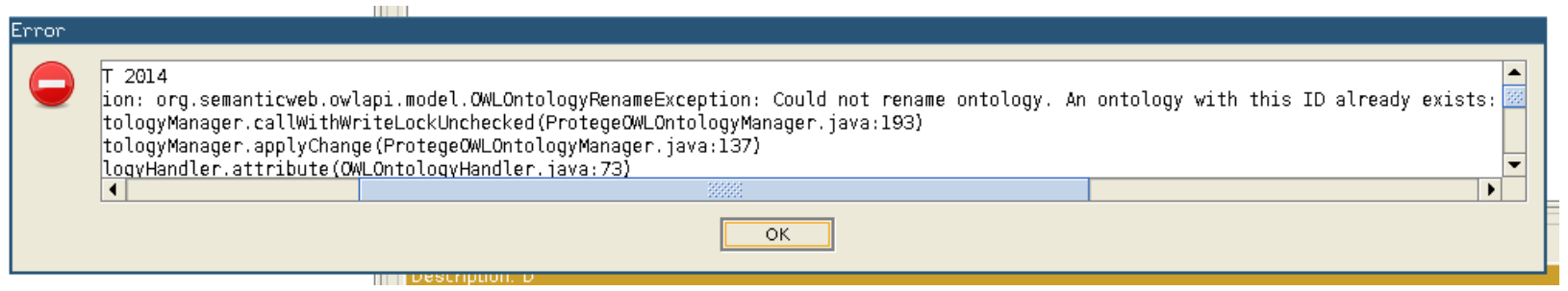

Figura A.22: Erro ao tentar realizar Fusão de ontologias com mesmo IRI

Assim, a ferramenta impossibilita a fusão de versões de uma mesma ontologia. A funcionalidade não realiza nenhuma espécie de casamento entre conceitos ao realizar fusão de ontologias, i.e., duas ontologias com IRIs diferentes, porém com classes com o mesmo nome, ao sofrerem fusão geram uma nova ontologia, que representa exatamente o conjunto de axiomas da ontologia 1 somado com o conjunto de axiomas da ontologia 2. Como a tecnologia OWL define espaços de nomes para cada conceito definido, a partir do IRI da ontologia, então, conceitos com mesmo nome não representam os mesmos conceitos pois a soma do nome com o IRI é diferente, e.g., ao realizar fusão de duas ontologias, $\mathcal{O}_{1}$ e $\mathcal{O}_{2}$ que possuem os IRIs: http://www.ime.usp.br/liamf/ontologies/o1 e http://www.ime.usp.br/liamf/ontologies/o2 - respectivamente, ambas possuindo o axioma $C \sqsubseteq D$, ao sofrerem fusão resultam em uma ontologia, que possui duas entradas para o axioma $C \sqsubseteq D$, uma para cada espaço de nomes.

Isso dificulta um pouco a tarefa de fusão, pois é necessário, para realizar fusão de axiomas que seus conceitos estejam no mesmo espaço de nomes, porém a ferramenta impossibilita o carregamento de duas ontologias com o mesmo IRI. Assim, só é possível realizar fusão de conceitos em duas ontologias onde a primeira utiliza os conceitos da segunda (mesmo espaço de nomes da segunda).

A fusão, nesse caso, se dá sem verificação, e.g., ao realizarmos a fusão de duas ontologias que possuem, os axiomas $D \sqsubseteq C$ e $D \sqsubseteq \neg C$ - respectivamente, a ferramenta não realiza nenhuma checagem de contradição entre os axiomas, prosseguindo com a cópia de ambos em uma nova ontologia, agora incoerente.

Por esse motivo, a ferramenta necessita que o editor possua uma funcionalidade de depuração de ontologias acoplado, para descobrir a causa das inferências indesejadas, que é o caso da ferramenta OWLExplanation plugin.

\section{A.2.3 Watson for Knowledge Reuse}

Watson for Knowledge Reuse [dSM08] é uma ferramenta implementada em Java e faz parte da suíte de desenvolvimento de ontologias NeonToolkit. Essa ferramenta tem como objetivo promover o reúso de conhecimento previamente definido e disponibilizado na web. Ela realiza, por meio do casamento léxico de conceitos, buscas em repositórios de ontologias - personalizado ou um catálogo específico da ferramenta - por conceitos já definidos e sugere ao usuário a inclusão de sub e superclasses. A Figura A.23 mostra a ferramenta sugerindo a inclusão da classe Faculty como super-classe de Professor. O conjunto de sugestões é visto no painel do lado direito, intitulado "Watson Results View". 


\begin{tabular}{|c|c|c|c|c|}
\hline $\mathcal{A}$ Ontology Navigator $\approx$ & 口曰 & \multirow{2}{*}{\multicolumn{3}{|c|}{ C Entity Properties Watson Results View $\mathbb{B}\left(\begin{array}{l}\text { Search results for: Professor } \\
\text { W }\end{array}\right.$}} \\
\hline \multirow{3}{*}{$\begin{array}{l}\nabla \text { Ontology1 [OWL2] } \\
\nabla<\text { ontology1 } \\
\nabla \backsim \text { Classes }\end{array}$} & & & & \\
\hline & & \multirow{4}{*}{\multicolumn{3}{|c|}{$\begin{array}{l}\text { Professor in http://cvs.suppressingfire.org/cgi-bin/viewcvs.cgi/*checkout*/cosi/112/pa1_2003031 } \\
\text { Professor in http://owl.man.ac.uk/2003/why/latest/facts.rdf } \\
\text { \ Professor in http://lsdis.cs.uga.edu/proj/sweto } \\
\text { จ Professor in http://ebiquity.umbc.edu/ontology/person.owl\#person }\end{array}$}} \\
\hline & & & & \\
\hline$\checkmark \mathbf{C}$ Professor & & & & \\
\hline \multirow{5}{*}{$\begin{array}{l}\text { C Role } \\
\square \text { Object Properties } \\
\square \text { Data Properties } \\
\square \text { Annotation Properties } \\
\square \text { Datatypes }\end{array}$} & & & & \\
\hline & & \multirow{2}{*}{$\begin{array}{l}\text { subclassof } \\
\text { label }\end{array}$} & \multirow{3}{*}{$\begin{array}{l}\text { Faculty } \\
\text { "Professor" }\end{array}$} & Add relation from Professor \\
\hline & & & & Add Literal in Professor \\
\hline & & \multicolumn{2}{|l|}{ View Ontology Summary } & \\
\hline & & \multicolumn{3}{|c|}{$\begin{array}{l}\triangleright \text { Professor in http://lsdis.cs.uga.edu/projects/semdis/sweto/testbed_v1_1.owl } \\
\triangleright \text { Professor in http://www.cs.man.ac.uk/ rector/CS646/Teaching-4-01-people-tangled.daml } \\
\text { \ Professor in http://www.daml.ri.cmu.edu/ont/homework/cmu-ri-employmenttypes-ont.daml }\end{array}$} \\
\hline
\end{tabular}

Figura A.23: Sugestão de inclusão de relação feita pela Watson

Essa ferramenta se mostra bastante útil no processo de definição inicial de uma ontologia, possibilitando que o usuário crie rapidamente hierarquias, tendo a possibilidade de acessar várias visões sobre como definir um dado conceito, examinando a estruturação das ontologias no repositório. O Watson ainda disponibiliza um conjunto de critérios que, se forem seguidos resultam em ontologias de maior qualidade. São eles:

- Verificar se o conceito a ser reusado vem de uma ontologia com escopo e enfase compatíveis;

- Verificar clareza do conceito que se está reutilizando;

- Verificar o conteúdo do que se está reutilizando. Verificar se a adição desse conteúdo será benéfica;

- Verificar de modo formal a ontologia após a inclusão do conceito e verificar se ela ainda é consistente;

- Verificar nomenclatura de indivíduos do tipo da classe recém-adicionada (nem sempre um nome copiado faz sentido);

Infelizmente, o Watson não provê nenhuma estratégia para a gerência de inconsistências. Se um usuário possui a ontologia mostrada na Figura A.24 e pede por sugestões sobre o conceito AssociateProfessor, o sistema então faz a sugestão que ele acrescente o conceito Professor como super-conceito, como pode ser visto na Figura A.25.

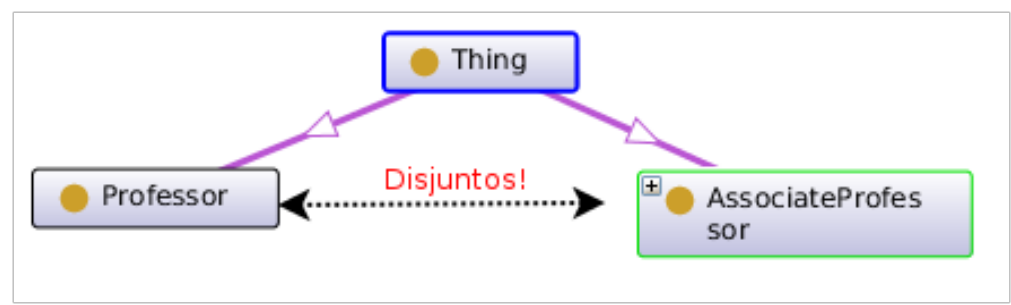

Figura A.24: Ontologia desenvolvida pelo usuário

A realização da operação sugerida pela ferramenta gera uma ontologia inconsistente. Portanto, a ferramenta não está integrada com nenhuma forma de reparo de ontologias pós fusão. 


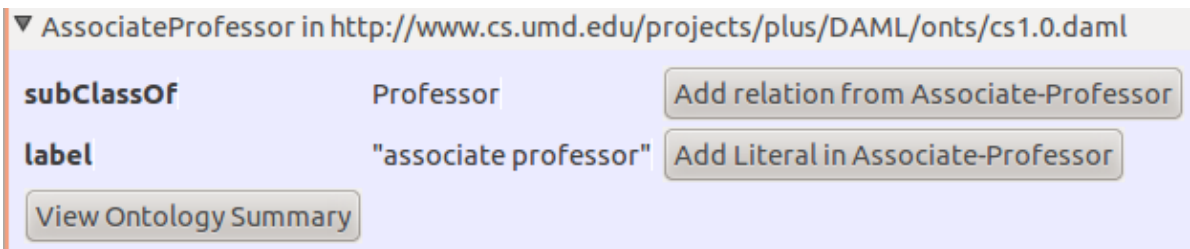

Figura A.25: Sugestão de inclusão de axioma à Ontologia desenvolvida pelo usuário

\section{A.2.4 OWLDiff}

A ferramenta OWLDiff realiza a comparação e fusão de ontologias, ajudando na gerência de modificações. A inspiração para a criação dessa ferramenta está em fazer uma ferramenta equivalente ao diff para arquivos de texto disponível no Unix. O seu processo de casamento de conceitos (diff entre ontologias) é feito pelo algoritmo CEX (complex effects of axiom modifications) [KWW08]. Um diferencial para essa ferramenta se encontra no fato de poder exibir ontologias utilizando a sintaxe padrão de Lógicas de Descrição, o que facilita a visualização das ontologias que estão sendo comparadas.

Um outro diferencial apresentado pelo OWLDiff é que possui uma versão standalone implementada em Java, utilizando OWLAPI para acesso e manipulação de ontologias, que pode ser utilizada sem necessidade de instalação de editores. Possui também versões disponíveis para serem integradas tanto ao NeonToolkit quanto ao Protégé e integração com sistemas de versionamento (Subversion).

Assim como nas outras ferramentas experimentadas, infelizmente o OWLDiff também não provê qualquer mecanismo de detecção e solução de inconsistências. Por exemplo, um usuário tenta realizar a operação de diff entre as ontologias apresentadas na Figura A.26.
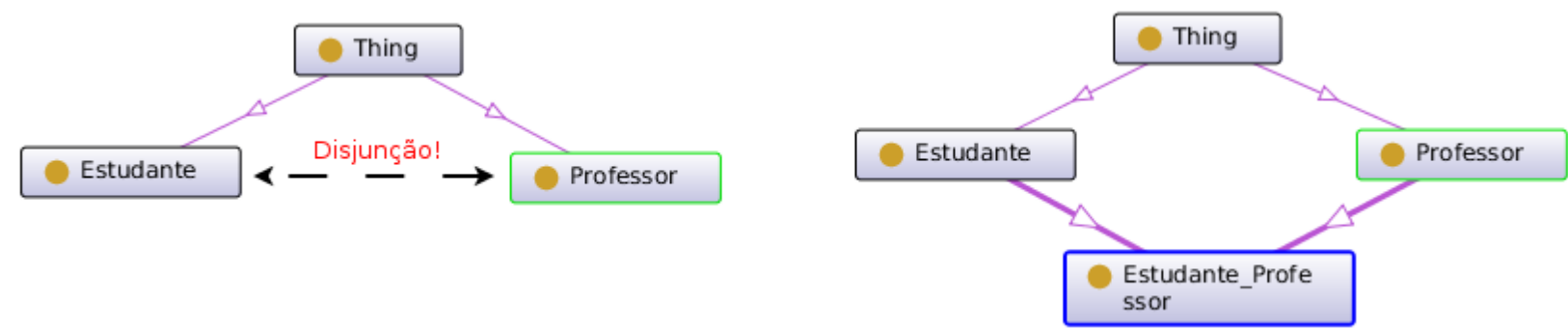

Figura A.26: Ontologias a passarem pela fusão utilizando OWLDiff

A ferramenta então mostra a diferença entre o conjunto de axiomas. O usuário então, decide que quer adicionar os axiomas Estudante_Professor $\sqsubseteq$ Estudante e Estudante_Professor $\sqsubseteq$ Professor da segunda ontologia a primeira, que contém o axioma Estudante $\sqcap$ Professor $=\emptyset$, o que claramente resulta em inconsistência, como pode ser visto na Figura A.27, onde o reasoner classifica o conceito Estudante_Professor como necessariamente vazio.

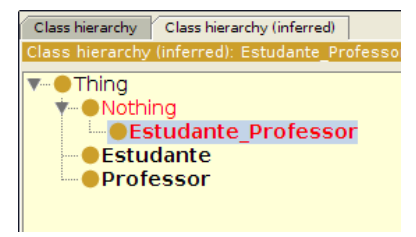

Figura A.27: Inconsistência gerada pela operação de fusão realizada pelo OWLDiff 


\section{A.2.5 OntoMerge}

OntoMerge $^{9}$ é uma ferramenta criada com o propósito de realizar a fusão de ontologias que utilizam a lógica DL-Lite ${ }^{\mathcal{N}}$. Por conta dessa redução na expressividade da linguagem utilizada para representar as ontologias, os autores podem utilizar estratégias definidas para lógicas proposicionais. Comumente essas estratégias são baseadas no cálculo e ordenação de modelos, porém, no caso da lógica de descrição, o número de modelos pode ser infinito. Portanto, os autores definiram uma nova caracterização semântica para a lógica DL-Lite [WWJQ12].

O OntoMerge não só é capaz de realizar a fusão de ontologias, mas também responder perguntas do tipo: $C \sqsubseteq D$ ?, mesmo antes de realizar a fusão. Assim, o sistema pode ser pensado como uma camada de ligação entre múltiplas ontologias, servindo como uma interface para várias fontes de dados com a capacidade de responder a perguntas sobre a taxonomia dessas fontes, de forma transparente.

Os autores argumentam que a fusão baseada somente na sintaxe não é suficiente para caracterizar a mudança mínima, uma vez que uma pequena mudança sintática pode impor grandes restrições ao domínio da função de interpretação para uma lógica de descrição. Assim, os autores definiram uma nova caracterização semântica e um mapeamento dessa para a lógica QBF - Quantified Boolean Formulas. A partir desse mapeamento é possível utilizar um conjunto de mecanismos de inferência já bem definidos e com desempenho melhor que o de mecanismo de inferência para lógica de descrição. Esse passo é o que garante a melhoria de desempenho quando é necessário qualquer raciocínio sobre uma base pós-fusão.

A abordagem utilizada pelos autores é deveras interessante, porém a restrição a um sub-conjunto muito específico da lógica de descrição restringe sua aplicabilidade em ontologias do mundo real. Porém, seria interessante conduzir estudos que comparassem o resultado da fusão utilizando a abordagem proposta e uma abordagem baseada na sintaxe.

\footnotetext{
${ }^{9}$ http://www.ict.griffith.edu.au/ kewen/OntoMerge/
} 


\section{Referências Bibliográficas}

[AGM85] Carlos E. Alchourrón, Peter Gärdenfors, and David Makinson. On the Logic of Theory Change: Partial Meet Contraction and Revision Functions. The Journal of Symbolic Logic, 50(2):510-530, 1985. 11, 16, 17, 45

[AM82] Carlos E. Alchourrón and David Makinson. On the logic of theory change: Contraction functions and their associated revision functions. Theoria, 48(1):14-37, 1982. 12

[BCD $\left.{ }^{+} 93\right] \quad$ Salem Benferhat, Claudette Cayrol, Didier Dubois, Jerome Lang, and Henri Prade. Inconsistency management and prioritized syntax-based entailment. In Proceedings of the 13th International Joint Conference on Artifical Intelligence - Volume 1, IJCAI'93, pages 640-645, San Francisco, CA, USA, 1993. Morgan Kaufmann Publishers Inc. 39

$\left[\mathrm{BCM}^{+}\right.$03] Franz Baader, Diego Calvanese, Deborah L. McGuinness, Daniele Nardi, and Peter F. Patel-Schneider, editors. The Description Logic Handbook: Theory, Implementation, and Applications. Cambridge University Press, New York, NY, USA, 2003. 6, 7, 29

[BEBC03] Salem Benferhat, Rania El Baida, and Frédéric Cuppens. A stratification-based approach for handling conflicts in access control. In Proceedings of the Eighth ACM Symposium on Access Control Models and Technologies, SACMAT '03, pages 189195, New York, NY, USA, 2003. ACM. 39, 41

[BFS13] Camila Bezerra, Fred Freitas, and Filipe Santana. Evaluating ontologies with competency questions. In Proceedings of the 2013 IEEE/WIC/ACM International Joint Conferences on Web Intelligence (WI) and Intelligent Agent Technologies (IAT) - Volume 03, WI-IAT '13, pages 284-285, Washington, DC, USA, 2013. IEEE Computer Society. 34

[BKLBW01] Salem Benferhat, Souhila Kaci, Daniel Le Berre, and Mary-Anne Williams. Weakening conflicting information for iterated revision and knowledge integration. In Proceedings of the 17th International Joint Conference on Artificial Intelligence - Volume 1, IJCAI'01, pages 109-115, San Francisco, CA, USA, 2001. Morgan Kaufmann Publishers Inc. 36

[BLW06] Piero A. Bonatti, Carsten Lutz, and Frank Wolter. Description logics with circumscription. In Proceedings, Tenth International Conference on Principles of Knowledge Representation and Reasoning, Lake District of the United Kingdom, pages 400-410, 2006. 60

[CMMV13] Giovanni Casini, Thomas Meyer, Kodylan Moodley, and Ivan J. Varzinczak. Towards practical defeasible reasoning for description logics. In Informal Proceedings of the 26th International Workshop on Description Logics, Ulm, Germany, July 23 - 26, 2013, pages 587-599, 2013. 60

[CRW13] Raphael Cóbe, Fillipe Resina, and Renata Wassermann. Merging ontologies via kernel contraction. In Proceedings of the Sixth ONTOBRAS-MOST, Belo Horizonte, Minas Gerais, Brazil, 2013, pages 94-105, 2013. 6 
[CW09] Raphael Cóbe and Renata Wassermann. Busca semântica baseada em ontologias. In Proceedings of the Second ONTOBRAS-MOST, Rio de Janeiro, Brazil, 2009. 6

[CW12] Raphael Cobe and Renata Wassermann. Ontology merging and conflict resolution: Inconsistency and incoherence solving approaches. Proceedings of the First Workshop on Belief change, Non-monotonic reasoning and Conflict resolution - BNC@ECAI (ECAI'12), Montpellier, France, page 20, 2012. 6

[CWK11] Raphael Cóbe, Renata Wassermann, and Fabio Kon. Ontology merging: on the confluence between theoretical and pragmatic approaches. In Proceedings of Third ONTOBRAS-MOST, Gramado, Rio Grande do Sul, Brazil, pages 159-164, 2011. 6

[Dal88] Mukesh Dalal. Updates in propositional databases. Technical Report DCS-TR-222, Department of Computer Science, Rutgers University, 1988. 2

[dSM08] Mathieu d'Aquin, Marta Sabou, and Enrico Motta. Reusing knowledge from the semantic web with the Watson plugin. In Demo session at the International Semantic Web Conference, ISWC, 2008. 121

[Flo06] Giorgos Flouris. On belief change in ontology evolution. AI Communications, 19(4):395-397, 2006. 29

[FNS07] Sean M. Falconer, Natalya F. Noy, and Margaret-Anne Storey. Ontology mapping a user survey. In Proceedings of the Workshop on Ontology Matching (OM2007) at ISWC/ASWC200\%, Busan, South Korea, 2007. 9

[FV04] Amir Fijany and Farokh Vatan. New approaches for efficient solution of hitting set problem. In Proceedings of the Winter International Symposium on Information and Communication Technologies, WISICT '04, pages 1-1. Trinity College Dublin, 2004. 29

[Gär88] Peter Gärdenfors. Knowledge in flux: Modeling the dynamics of epistemic states. The MIT press, 1988. 12

[GH08] Nikos Gorogiannis and Anthony Hunter. Merging first-order knowledge using dilation operators. In Proceedings of the 5th international conference on Foundations of information and knowledge systems, FoIKS'08, pages 132-150, Berlin, Heidelberg, 2008. Springer-Verlag. 2, 36

[Han91] Sven O. Hansson. Belief contraction without recovery. Studia Logica, 50(2):251-260, 1991. 11

[Han93] Sven O. Hansson. Reversing the Levi identity. Journal of Philosophical Logic, 22(6):637-669, 1993. 13

[Han94] Sven O. Hansson. Kernel contraction. The Journal of Symbolic Logic, 59(03):845-859, 1994. 14,15

[Har86] Gilbert Harman. Change in view: Principles of reasoning. MIT Press, 1986. 12

[HCdK92] Walter Hamscher, Luca Console, and Johan de Kleer, editors. Readings in Modelbased Diagnosis. Morgan Kaufmann Publishers Inc., San Francisco, CA, USA, 1992. 24

[Hor11] Matthew Horridge. Justification based explanation in ontologies. PhD thesis, the University of Manchester, 2011. 2, 3, 18, 22, 24, 25, 30, 35, 36, 38, 45, 54, 55, 56, 57, $58,70,72,73,75,91,112,115$ 
[HPS08] Matthew Horridge, Bijan Parsia, and Ulrike Sattler. Explanation of OWL entailments in Protégé 4. In International Semantic Web Conference (Posters \& Demos), volume 401, 2008. 18, 30

[HS05] Peter Haase and Ljiljana Stojanovic. Consistent evolution of OWL ontologies. In Asunción Gómez-Pérez and Jérôme Euzenat, editors, The Semantic Web: Research and Applications, volume 3532 of Lecture Notes in Computer Science, pages 91-133. Springer Berlin / Heidelberg, 2005. 34

[HV08] Peter Haase and Johanna Volker. Ontology learning and reasoning — dealing with uncertainty and inconsistency. In Paulo da Costa, Claudia d'Amato, Nicola Fanizzi, Kathryn Laskey, Kenneth Laskey, Thomas Lukasiewicz, Matthias Nickles, and Michael Pool, editors, Uncertainty Reasoning for the Semantic Web I, volume 5327 of Lecture Notes in Computer Science, pages 366-384. Springer Berlin / Heidelberg, 2008. 36

[HvHH ${ }^{+}$05] Peter Haase, Frank van Harmelen, Zhisheng Huang, Heiner Stuckenschmidt, and York Sure. A framework for handling inconsistency in changing ontologies. In Proceedings of the Fourth Internation Semantic Web Conference, volume 3729 of LNCS, pages 353-367. Springer, 2005. 1, 2, 4, 7, 19, 34, 36

[HvHtT05] Zhisheng Huang, Frank van Harmelen, and Annette ten Teije. Reasoning with inconsistent ontologies. In IJCAI-05, Proceedings of the Nineteenth International Joint Conference on Artificial Intelligence, Edinburgh, Scotland, UK, July 30-August 5, 2005, pages 454-459, 2005. 20, 21

[HW02] Sven O. Hansson and Renata Wassermann. Local change. Studia Logica, 70(1):49-76, 2002. 16,18

[HWKP06] Christian Halaschek-Wiener, Yarden Katz, and Bijan Parsia. Belief base revision for expressive description logics. In $O W L E D$, volume 216, 2006. 18

[JHQ ${ }^{+}$09] Qiu Ji, Peter Haase, Guilin Qi, Pascal Hitzler, and Steffen Stadtmüller. Radon repair and diagnosis in ontology networks. In Lora Aroyo, Paolo Traverso, Fabio Ciravegna, Philipp Cimiano, Tom Heath, Eero Hyvönen, Riichiro Mizoguchi, Eyal Oren, Marta Sabou, and Elena Simperl, editors, The Semantic Web: Research and Applications, volume 5554 of Lecture Notes in Computer Science, pages 863-867. Springer Berlin Heidelberg, 2009. 18, 108

[JQH09] Qiu Ji, Guilin Qi, and Peter Haase. A relevance-directed algorithm for finding justifications of DL entailments. In Asunción Gómez-Pérez, Yong Yu, and Ying Ding, editors, The Semantic Web, volume 5926 of Lecture Notes in Computer Science, pages 306-320. Springer Berlin Heidelberg, 2009. 2, 20

[Jun01] Ulrich Junker. Quickxplain: Conflict detection for arbitrary constraint propagation algorithms. In Proceedings of the Workshop on Modelling and Solving problems with constraints (IJCAI'01), Seattle, WA, USA. Morgan Kaufmann Publishers Inc., 2001. 23,67

[Kal06] Aditya A. Kalyanpur. Debugging and repair of OWL ontologies. PhD thesis, the University of Maryland, 2006. 2, 3, 4, 15, 17, 18, 22, 23, 24, 25, 34, 35, 36, 41, 45, 52, $55,56,57,58,65,106,108$

[Kon00] Sebastien Konieczny. On the difference between merging knowledge bases and combining them. In Seventh International Conference on Principles of Knowledge Representation and Reasoning (KR'00), pages 135-144, 2000. 1, 2 
[KPH05] Aditya A. Kalyanpur, Bijan Parsia, and James Hendler. A tool for working with web ontologies. International Journal on Semantic Web and Information Systems (IJSWIS), 1(1):36-49, 2005. 18

[KPHS07] Aditya A. Kalyanpur, Bijan Parsia, Matthew Horridge, and Evren Sirin. Finding all justifications of OWL DL entailments. In Karl Aberer, Key-Sun Choi, Natasha Noy, Dean Allemang, Kyung-Il Lee, Lyndon Nixon, Jennifer Golbeck, Peter Mika, Diana Maynard, Riichiro Mizoguchi, Guus Schreiber, and Philippe Cudré-Mauroux, editors, The Semantic Web, volume 4825 of Lecture Notes in Computer Science, pages 267-280. Springer Berlin Heidelberg, 2007. 2, 20, 22, 30

[KPP98] Sébastian Konieczny and Ramon Pino-Pérez. On the logic of merging. In Sixth International Conference on Principles of Knowledge Representation and Reasoning (KR'98), pages 488-498, 1998. 1

[KPP99] Sébastian Konieczny and Ramon Pino-Pérez. Merging with integrity constraints. In Fifth European Conference on Symbolic and Quantitative Approaches to Reasoning with Uncertainty (ECSQARU'99), pages 233-244, 1999. 1

[KWW08] Boris Konev, Dirk Walther, and Frank Wolter. The logical difference problem for description logic terminologies. In Proceedings of Automated Reasoning, 4th International Joint Conference, IJCAR 2008, Sydney, Australia, pages 259-274, 2008. 123

[LBF $\left.{ }^{+} 06\right] \quad$ Carsten Lutz, Franz Baader, Enrico Franconi, Domenico Lembo, Ralf Möller, Riccardo Rosati, Ulrike Sattler, Boontawee Suntisrivaraporn, and Sergio Tessaris. Reasoning support for ontology design. In Proceedings of the OWLED 06 Workshop on OWL: Experiences and Directions, 2006. 34

[LPSV06] Joey Lam, Jeff Z. Pan, Derek Sleeman, and Wamberto Vasconcelos. A fine-grained approach to resolving unsatisfiable ontologies. In IEEE/WIC/ACM International Conference on Web Intelligence, 2006. WI 2006., pages 428 -434, dec. 2006. 35, 36

[LRW12] Renato U. Lundberg, Márcio M. Ribeiro, and Renata Wassermann. A framework for empirical evaluation of belief change operators. In Leliane N. Barros, Marcelo Finger, Aurora T. Pozo, Gustavo A. Gimenénez-Lugo, and Marcos Castilho, editors, Advances in Artificial Intelligence - SBIA 2012, Lecture Notes in Computer Science, volume 7589, pages 12-21. Springer Berlin Heidelberg, 2012. 6

[LS95] Paolo Liberatore and Marco Schaerf. Arbitration: A commutative operator for belief revision. In Proceedings of the Second World Conference on the Fundamentals of Artificial Intelligence (WOCFAI'95), pages 217-228, 1995. 48

[LS98] Paolo Liberatore and Marco Schaerf. Arbitration (or how to merge knowledge bases). Knowledge and Data Engineering, IEEE Transactions on, 10(1):76 -90, jan/feb 1998. 48

[MLB05] Thomas Meyer, Kevin Lee, and Richard Booth. Knowledge integration for description logics. In Proceedings of the 20th National Conference on Artificial Intelligence Volume 2, AAAI'05, pages 645-650. AAAI Press, 2005. 2, 6, 8, 9, 34, 36, 38, 39, 54

[MLBP06] Thomas Meyer, Kevin Lee, Richard Booth, and Jeff Z. Pan. Finding maximally satisfiable terminologies for the description logic ALC. In Proceedings of the 21st National Conference on Artificial Intelligence - Volume 1, AAAI'06, pages 269-274. AAAI Press, 2006. 34, 36, 39 
[MMV11] Kodylan Moodley, Thomas Meyer, and Ivan J. Varzinczak. Root justifications for ontology repair. In Sebastian Rudolph and Claudio Gutierrez, editors, Web Reasoning and Rule Systems, volume 6902 of Lecture Notes in Computer Science, pages 275-280. Springer Berlin Heidelberg, 2011. 30

[MP98] Deborah L. McGuinness and Peter F. Patel-Schneider. Usability issues in knowledge representation systems. In Proceedings of the Fifteenth National Conference on Artificial Intelligence and Tenth Innovative Applications of Artificial Intelligence Conference, AAAI 98, IAAI 98, Madison, Wisconsin, USA., pages 608-614, 1998. 34

[NH97] Natalya F. Noy and Carole D. Hafner. The state of the art in ontology design: A survey and comparative review. AI Magazine, 18(3):53-74, 1997. 34

[NM00] Natalya F. Noy and Mark A. Musen. Algorithm and tool for automated ontology merging and alignment. In Proceedings of the 17th National Conference on Artificial Intelligence (AAAI-00). Available as SMI technical report SMI-2000-0831, 2000. 115

[NM01] Natalya F. Noy and Mark Musen. Anchor-PROMPT: Using non-local context for semantic matching. In Workshop on Ontologies and Information Sharing at the 17th International Joint Conference on Artificial Intelligence (IJCAI-2001), Seattle, WA, USA, pages 63-70, 2001. 115

[NM03] Natalya F. Noy and Mark A. Musen. The PROMPT suite: interactive tools for ontology merging and mapping. International Journal of Human-Computer Studies, 59(6):983-1024, 2003. 35, 115

[PG86] David A. Plaisted and Steven Greenbaum. A structure-preserving clause form translation. Journal of Symbolic Computation, 2(3):293-304, 1986. 57

[PSK05] Bijan Parsia, Evren Sirin, and Aditya A. Kalyanpur. Debugging OWL ontologies. In Proceedings of the 14th international conference on World Wide Web, pages 633-640. ACM, 2005. 38

[QLB06] Guilin Qi, Weiru Liu, and David Bell. A revision-based approach to handling inconsistency in description logics. Artificial Intelligence Review, 26(1-2):115-128, 2006. 2, $3,4,8,35,36,39,54,59,62$

[QP07] Guilin Qi and Jeff Z. Pan. A stratification-based approach for inconsistency handling in description logics. In International Workshop on Ontology Dynamics (IWOD-07), page 83, Innsbruck, Austria, 2007. 4, 6, 7, 8, 35, 39, 40, 45, 46, 52

[Rei87] Raymond Reiter. A theory of diagnosis from first principles. Artificial intelligence, 32(1):57-95, 1987. 2, 24, 32, 72

[Rev93] Peter Z. Revesz. On the semantics of theory change: arbitration between old and new information. In Proceedings of the twelfth ACM SIGACT-SIGMOD-SIGART symposium on Principles of database systems, PODS '93, pages 71-82, New York, NY, USA, 1993. ACM. 1, 2, 9

[Rev97] Peter Z. Revesz. On the semantics of arbitration. International Journal of Algebra and Computation, 7(2):133-160, 1997. 1, 9

[Rib13] Márcio M. Ribeiro. Belief revision in non-classical logics. Springer, 2013. 12, 13, 14, 29,30

[RRW14] Fillipe Resina, Márcio M. Ribeiro, and Renata Wassermann. Algorithms for multiple contraction and an application to OWL ontologies. In Proceedings of the Brazilian Conference on Intelligent Systems (BRACIS), 2014. 2, 6, 30, 31, 32, 68, 72, 73, 76, 100 
[RW08] Márcio M. Ribeiro and Renata Wassermann. On the relation between remainder sets and kernels. XV Encontro Brasileiro de Lógica (EBL08), Paraty, RJ, Brasil, 2008. 29

[Sch05] Stefan Schlobach. Debugging and semantic clarification by pinpointing. In Asunción Gómez-Pérez and Jérôme Euzenat, editors, The Semantic Web: Research and Applications, volume 3532 of Lecture Notes in Computer Science, pages 27-44. Springer Berlin / Heidelberg, 2005. 34, 36

[SFJ08] Kostyantyn Shchekotykhin, Gerhard Friedrich, and Dietmar Jannach. On computing minimal conflicts for ontology debugging. In MBS 2008 - Workshop on Model Based Systems at ECAI 2008, Patras. Greece, pages 7-12, 2008. 24

[SHCvH07] Stefan. Schlobach, Zhisheng Huang, Ronald Cornet, and Frank van Harmelen. Debugging incoherent terminologies. Journal of Automated Reasoning, 39:317-349, 2007. 10.1007/s10817-007-9076-z. 2, 34

[SQJH08] Boontawee Suntisrivaraporn, Guilin Qi, Qiu Ji, and Peter Haase. A modularizationbased approach to finding all justifications for OWL DL entailments. In John Domingue and Chutiporn Anutariya, editors, The Semantic Web, volume 5367 of Lecture Notes in Computer Science, pages 1-15. Springer Berlin Heidelberg, 2008. 2, 20

[Sun09] Boontawee Suntisrivaraporn. Polynomial time reasoning support for design and maintenance of large-scale biomedical ontologies. PhD thesis, Dresden University of Technology, 2009. 19

[VPSS10] Chiara Del Vescovo, Bijan Parsia, Ulrike Sattler, and Thomas Schneider. The modular structure of an ontology: an empirical study. In Volker Haarslev, David Toman, and Grant E. Weddell, editors, Description Logics, volume 573 of CEUR Workshop Proceedings. CEUR-WS.org, 2010. 22

[Was99] Renata Wassermann. Resource-Bounded Belief Revision. PhD thesis, Institute for Logic, Language and Computation - University of Amsterdam, 1999. 2, 66

[Was00] Renata Wassermann. An algorithm for belief revision. In KR 2000, Principles of Knowledge Representation and Reasoning Proceedings of the Seventh International Conference, Breckenridge, Colorado, USA, pages 345-352, 2000. 18, 21, 24, 25, 72

[Was01] Renata Wassermann. On structured belief bases. In Hans Rott and Mary-Anne Williams, editors, Frontiers in Belief Revision. Kluwer, 2001. 54

[WWJQ12] Zhe Wang, Kewen Wang, Yifan Jin, and Guilin Qi. Ontomerge: A system for merging DL-Lite ontologies. In Proceedings of the Eighth Australasian Ontology Workshop, Sydney, Australia, 2012. 124 